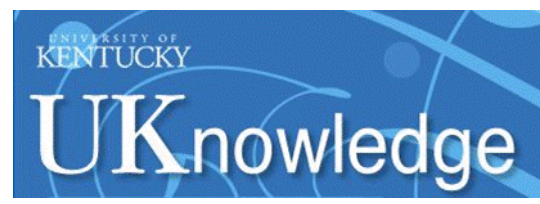

University of Kentucky

UKnowledge

2012

\title{
VALIDATION OF Fhb1 AND QFhs.nau-2DL IN SEVERAL SOFT RED WINTER WHEAT POPULATIONS
}

Ana L. Balut

University of Kentucky, ana.balut@uky.edu

Right click to open a feedback form in a new tab to let us know how this document benefits you.

\section{Recommended Citation}

Balut, Ana L., "VALIDATION OF Fhb1 AND QFhs.nau-2DL IN SEVERAL SOFT RED WINTER WHEAT POPULATIONS" (2012). Theses and Dissertations--Plant and Soil Sciences. 14.

https://uknowledge.uky.edu/pss_etds/14

This Master's Thesis is brought to you for free and open access by the Plant and Soil Sciences at UKnowledge. It has been accepted for inclusion in Theses and Dissertations--Plant and Soil Sciences by an authorized administrator of UKnowledge. For more information, please contact UKnowledge@lsv.uky.edu. 


\section{STUDENT AGREEMENT:}

I represent that my thesis or dissertation and abstract are my original work. Proper attribution has been given to all outside sources. I understand that I am solely responsible for obtaining any needed copyright permissions. I have obtained and attached hereto needed written permission statements(s) from the owner(s) of each third-party copyrighted matter to be included in my work, allowing electronic distribution (if such use is not permitted by the fair use doctrine).

I hereby grant to The University of Kentucky and its agents the non-exclusive license to archive and make accessible my work in whole or in part in all forms of media, now or hereafter known. I agree that the document mentioned above may be made available immediately for worldwide access unless a preapproved embargo applies.

I retain all other ownership rights to the copyright of my work. I also retain the right to use in future works (such as articles or books) all or part of my work. I understand that I am free to register the copyright to my work.

\section{REVIEW, APPROVAL AND ACCEPTANCE}

The document mentioned above has been reviewed and accepted by the student's advisor, on behalf of the advisory committee, and by the Director of Graduate Studies (DGS), on behalf of the program; we verify that this is the final, approved version of the student's dissertation including all changes required by the advisory committee. The undersigned agree to abide by the statements above.

Ana L. Balut, Student

Dr. David A. Van Sanford, Major Professor

Dr. Dennis B. Egli, Director of Graduate Studies 
VALIDATION OF Fhb1 AND $Q F h s . n a u-2 D L$ IN SEVERAL SOFT RED WINTER WHEAT POPULATIONS

\section{THESIS}

A thesis submitted in partial fulfillment of the requirements for the degree of Master of Science in the

College of Agriculture

at the University of Kentucky

By

Ana L. Balut

Director: Dr. David A. Van Sanford, Professor of Agronomy

Lexington, Kentucky

2012

Copyright (C) Ana L. Balut 2012 


\begin{abstract}
OF THESIS
VALIDATION OF Fhbl AND QFhs.nau-2DL IN SEVERAL SOFT RED WINTER WHEAT POPULATIONS
\end{abstract}

The use of exotic resistance quantitative trait loci (QTL) provides one strategy for breeding wheat cultivars resistant to Fusarium Head Blight (FHB), a devastating disease of wheat. The objective of this study was to evaluate the effectiveness of two QTL, Fhb1 and QFhs.nau-2DL, in diverse genetic backgrounds and to evaluate their effects on agronomic and quality traits. Five populations from crosses between FHB susceptible parents (26R58, KY97C-0574-01, 25R54, KY97C, KY97C-0554-02, 25R78 and KY93C-1238-17-1) and FHB-resistant VA01W-476, were evaluated in the FHB nursery at Lexington, $\mathrm{KY}$ in 2010 and 2011. The populations were also grown in yield trials at Lexington (2010 and 2011) and Princeton (2011), KY, to measure agronomic and quality traits. Fhb1 reduced Fusarium damaged kernels (FDK) by $32 \%$ and the toxin, deoxynivalenol (DON) by 20\%. QFhs.nau-2DL significantly reduced mean FDK by $29 \%$ in two of five populations and DON by $24 \%$ in four of five populations. While the effects of these QTL on agronomic and quality traits were significant, the impact was small. One cycle of either direct or indirect simulated phenotypic selection was effective at reducing DON levels and the frequency of Fhbl-homozygous resistant lines among the selects was higher than the frequency of $Q F h s$.nau- $2 D L$-homozygous resistant lines.

Keywords: Triticum aestivum, Deoxynivalenol, Fusarium head blight, Wheat quality, Wheat breeding

Ana L. Balut

July 12, 2012 


\section{VALIDATION OF Fhb1 AND QFhs.nau-2DL IN SEVERAL SOFT RED WINTER WHEAT POPULATIONS}

By

Ana L. Balut

Dr. David A. Van Sanford Director of Thesis

Dr. Dennis B. Egli Director of Graduate Studies

July 12, 2012 


\section{ACKNOWLEDGMENTS}

I would like to express my gratitude to Dr. David Van Sanford, my major professor, for mentoring and inspiring me. His guidance, words of wisdom and support have been of great importance and fundamental for me to complete this work. I would also like to thank Dr. Timothy Phillips and Dr. Donald Hershman, members of my advisory committee for their contributions.

My gratitude is also extended to past and present Wheat Breeding Program members for their help and assistance throughout this project. Accomplishing my entire field work was only possible with their help, ideas and enthusiasm. I particularly want to thank Dr. Clark for his help in both field and lab work, along with Sandy Swanson and John Connelly. Special thanks to my fellow graduate student Daniela Sarti, for her constant cooperation and friendship during these years.

I would like to express my deepest thanks to Martin Navarro, my love and best

friend, who supported me during this process and made helpful contributions. Finally, my appreciation is extended to my family and friends in Argentina and Lexington for their love and support. 


\section{TABLE OF CONTENTS}

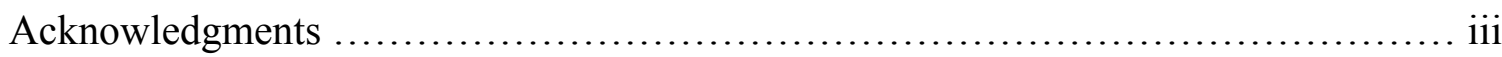

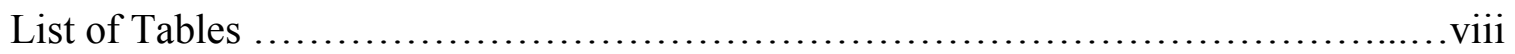

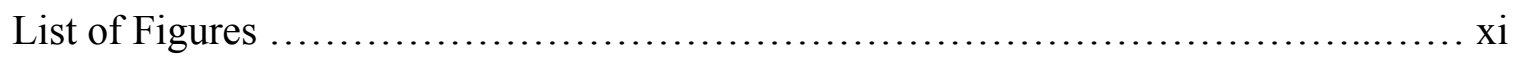

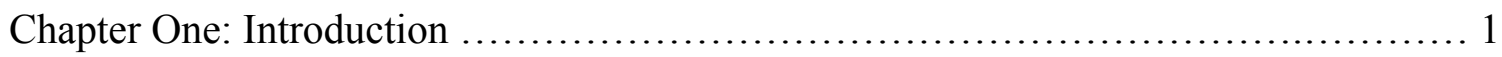

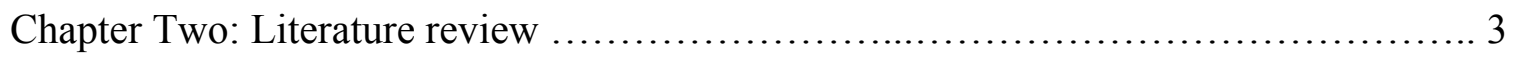

Economic impact of Fusarium Head Blight ............................... 3

Pathology and epidemiology of Fusarium graminearum ..................... 4

Host resistance to Fusarium Head Blight: mechanisms and genetic sources of

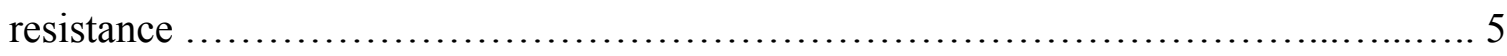

Breeding for Fusarium Head Blight resistance: conventional breeding and marker

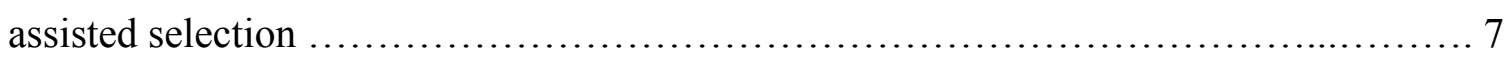

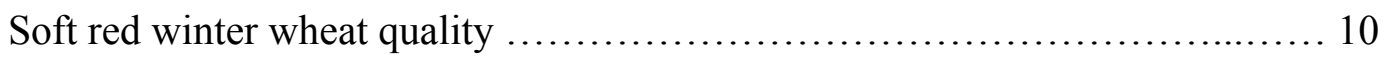

Use of near infrared reflectance to measure FHB and quality traits ............ 12 Chapter Three: Effects of Fhbl and QFhs.nau-2DL on FHB, agronomic and quality traits

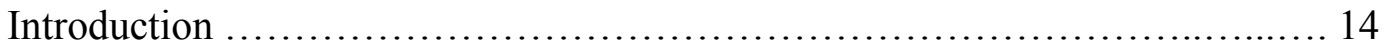

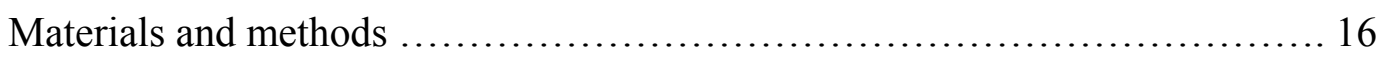

Plant material ............................................... 16

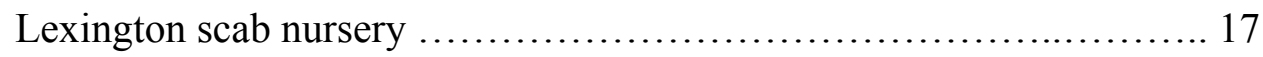

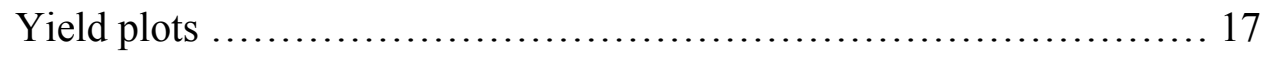

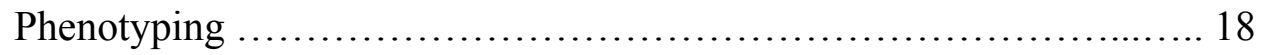


Data analysis .............................................. 19

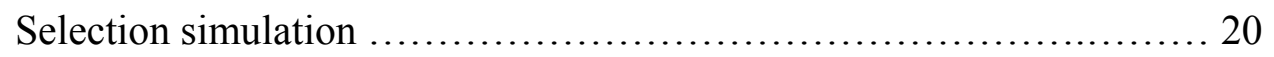

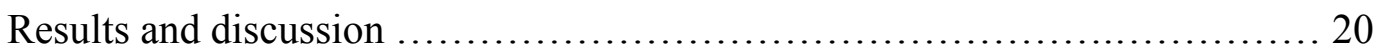

Weather conditions and disease levels .......................... 20

Fhbl effects on FHB traits .................................... 21

QFhs.nau-2DL effects on FHB traits ........................... 22

Agronomic traits. ........................................... 22

Fhbl effects on agronomic traits ......................... 23

QFhs.nau-2DL effects on agronomic traits ................ 23

Fhbl effects on milling and baking quality ...................... 23

QFhs.nau-2DL effects on milling and baking quality $\ldots \ldots \ldots \ldots \ldots \ldots 24$

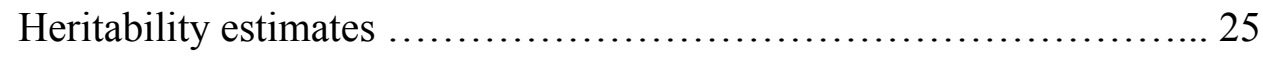

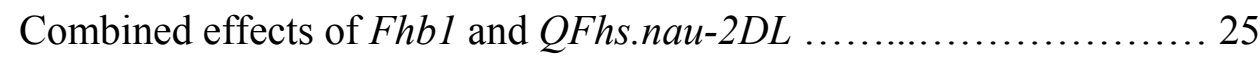

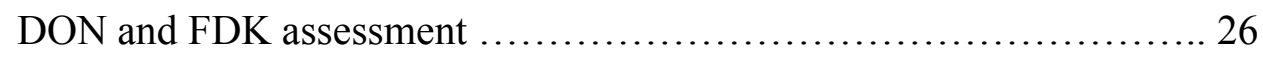

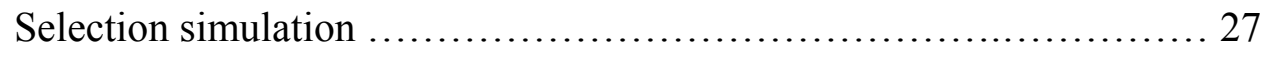

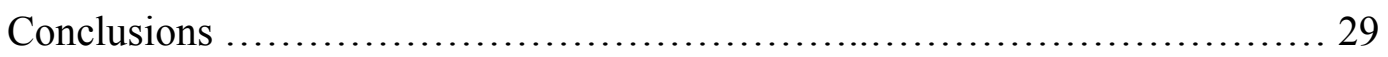

Chapter Four: Milling and baking quality predictions using wheat meal assays and near infrared reflectance ....................................................... 40

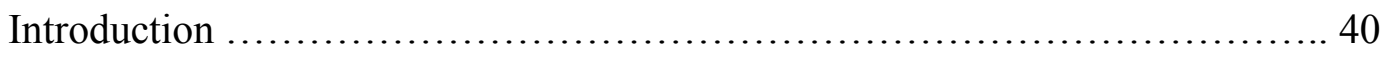

Materials and methods ............................................ 42

Plant material ............................................... 42 
Phenotyping .................................................... 43

Traditional methods ........................................... 43

Wheat meal SDS sedimentation volume ....................... 44

Wheat meal sodium carbonate SRC ......................... 44

Near infrared reflectance .................................... 45

Data analysis ................................................... 45

Relationship between wheat meal and NIR based predictions with

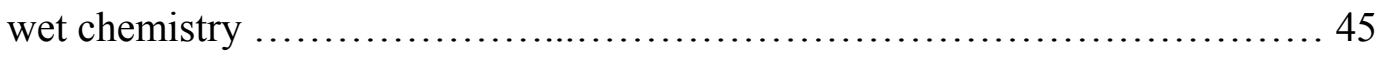

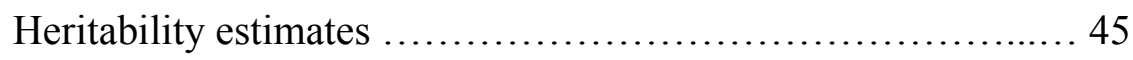

Selection simulation ...................................... 46

Results and Discussion ............................................... 46

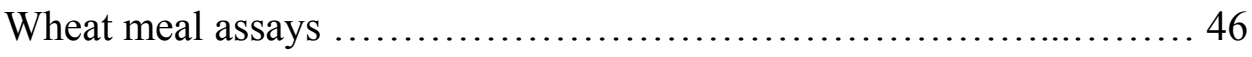

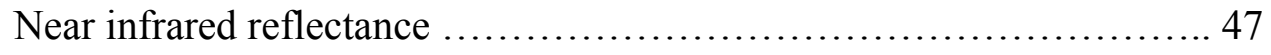

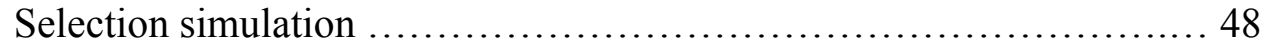

Wheat meal assays ........................................... 48

Near infrared reflectance ...................................... 49

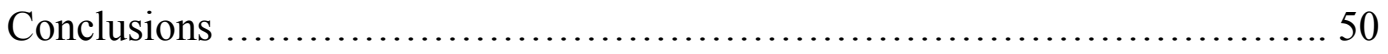

Chapter Five: Prediction of FDK and DON using near infrared reflectance ............ 63

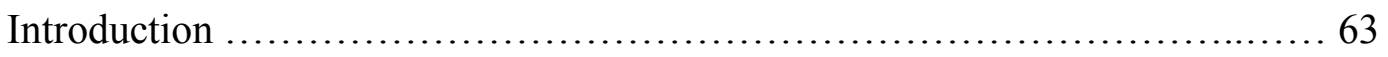

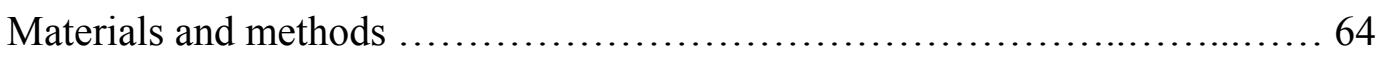

Plant material ......................................................... 64 


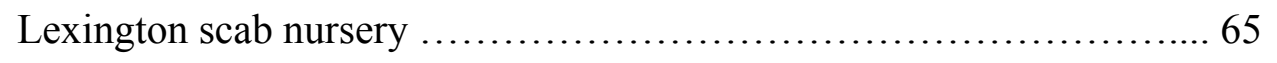

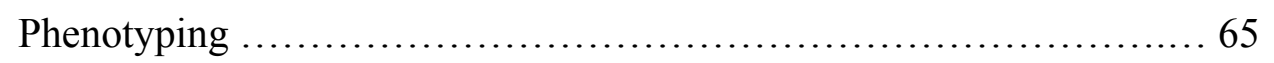

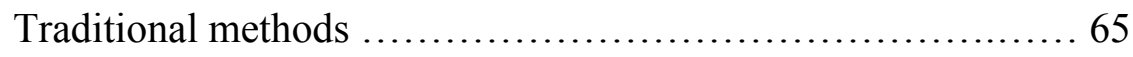

Near infrared reflectance .................................66

Data analysis............................................... 66

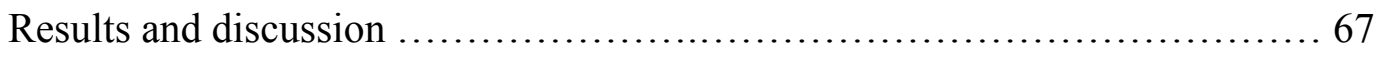

Heritability of FDK and DON measured using NIR ................. 67

Relationship between FHB traits .............................. 68

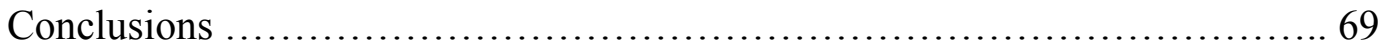

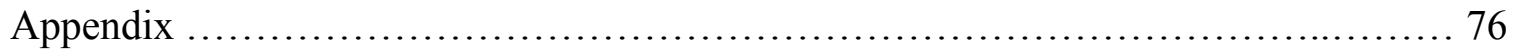

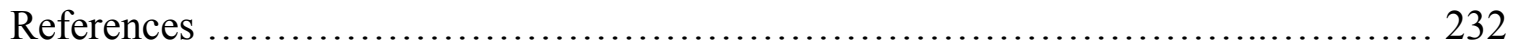

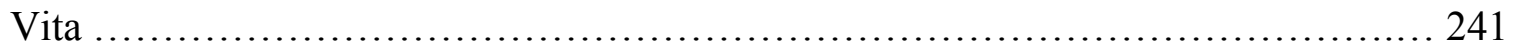




\section{LIST OF TABLES}

Table 3.1: Mean, Maximum (Max) and Minimum (Min) Fusarium damaged kernels (FDK), deoxynivalenol level (DON), and FHB index for five wheat populations and their parents in the Lexington, KY scab nursery in 2010 and 2011 ....................... 30

Table 3.2: Mean Fusarium damaged kernels (FDK), deoxynivalenol levels (DON), FHB index, yield, test weight (TWT), flour yield (FY), softness equivalent (SEQ) and gluten performance index (GPI) for wheat lines homozygous resistant (R) and susceptible (S) alleles at Fhb1, Lexington 2010 and 2011, and Princeton 2011.

Table 3.3: Mean Fusarium damaged kernels (FDK), deoxynivalenol levels (DON), FHB index, yield, test weight (TWT), flour yield (FY), softness equivalent (SEQ) and gluten performance index (GPI) for homozygous susceptible (S), resistant (R) and heterozygous (H) wheat lines at QFhs.nau-2DL, Lexington 2010 and 2011, and Princeton 2011.

Table 3.4: Mean, Maximum (Max) and Minimum (Min) yield and test weight (TWT) for five wheat populations and their parents in Lexington (LEX) 2010 and 2011, and Princeton (PRN) 2011. Mean flour yield (FY), softness equivalent (SEQ) and gluten performance index (GPI) for LEX 2010 and PRN 2011.

Table 3.5: Heritabilities and their 90\% confidence intervals in parentheses, based on 2 year ANOVA of five wheat populations. Traits evaluated were: Fusarium damaged kernels (FDK), deoxynivalenol level (DON), FHB index, flour yield (FY), softness equivalent (SEQ) and gluten performance index (GPI), Lexington and Princeton, KY 2010-2011.

Table 3.6: Mean deoxynivalenol (DON) reduction after one cycle of simulated selection, proportion of the population selected (p), number of lines selected (n) for different selection criteria, and lines selected from the following genotypic categories: resistant for both Fhbl and QFhs.nau-2DL (RR), resistant for Fhbl and heterozygous for QFhs.nau- 
$2 D L(\mathrm{RH})$, resistant for $F h b 1$ and susceptible for $Q F h$ s.nau-2DL (RS), susceptible for Fhb1 and R for QFhs.nau-2DL (SR), susceptible for both Fhb1 and QFhs.nau-2DL (SS), resistant for $F h b 1$ and unknown for $Q F h s . n a u-2 D L$ (R?), and $\mathrm{S}$ for $F h b 1$ and unknown for QFhs.nau-2DL (S?).

Table 4.1: Mean, Maximum (Max) and Minimum (Min) wheat meal SDS sedimentation (WM-SDS) and wheat meal sodium carbonate SRC (WM-SCSRC) for five wheat populations and their parents in Lexington (LEX) 2010 and Princeton (PRN) 2011.

Table 4.2: Genotype mean correlations for whole grain protein, whole grain hardness, flour yield, softness equivalent, flour protein, flour solvent retention capacity profile (SRC), gluten performance index (GPI) and estimated cookie diameter of 155 wheat lines with wheat meal SDS sedimentation (WM-SDS) and WM-sodium carbonate SRC in Lexington 2010 and Princeton 2011.

Table 4.3: Genotype mean correlation for whole grain NIR predictions and flour and wheat meal based quality parameters in Lexington 2010 and Princeton 2011.

Table 4.4: Wheat meal sedimentation (WM-SDS), WM-sodium carbonate SRC, flour lactic acid SRC, flour sodium carbonate SRC and flour yield heritabilities and their 90\% confidence intervals in parentheses, based on a 2 year ANOVA of five wheat populations evaluated in Lexington 2010 and Princeton 2011.

Table 4.5: Mean flour lactic acid SRC increment after one cycle of direct and indirect simulated selection, proportion of the population selected $(\mathrm{p})$ and number of lines selected (a), and mean flour sodium carbonate SRC, flour yield and flour sucrose SRC reduction after one cycle of direct and indirect simulated selection, proportion of the population selected and number of lines selected (b).

Table 4.6: Number of lines retained when the highest 50 and $25 \%$ of the population were selected for gluten strength based on wheat meal sedimentation (WM-SDS) as compared 
with the number of lines retained when the highest $25 \%$ of the population was selected

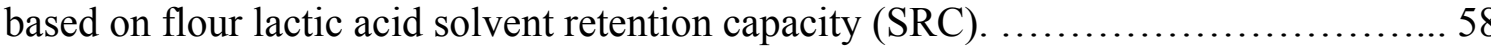

Table 4.7: Number of lines retained when the highest 50 and $25 \%$ of the population were selected for flour yield based on the lowest wheat meal (WM) sodium carbonate solvent retention capacity (SRC) as compared with the number of lines retained when the highest $25 \%$ of the population was selected based on flour yield.

Table 4.8: Number of lines retained when the highest 50 and $25 \%$ of the population were selected for softness equivalent based on whole grain NIR softness equivalent as compared with the number of lines retained when the highest $25 \%$ of the population was selected based on softness equivalent.

Table 4.9: Number of lines retained when the highest 50 and $25 \%$ of the population were selected for flour yield based on whole grain NIR flour yield as compared with the number of lines retained when the highest $25 \%$ of the population was selected based on flour yield.

Table 4.10: Number of lines retained when the lowest 50 and $25 \%$ of the population were selected for flour water solvent retention capacity (SRC) based on whole grain NIR water SRC as compared with the number of lines retained when the lowest $25 \%$ of the population was selected based on flour water SRC.

Table 5.1: Heritability and 90\% confidence interval estimates in parentheses for NIRFDK and NIRDON based on a 2 year ANOVA of five wheat populations evaluated in Lexington 2010 and Princeton 2011 70

Table 5.2: Genotype mean Pearson correlation coefficients for FHB traits and NIR measurements (NIRFDK, NIRDON) for all five populations in Lexington 2010 and 2011. 


\section{LIST OF FIGURES}

Figure 3.1: Relationship between 2010 and 2011 measurements of FDK (a) and DON (b)

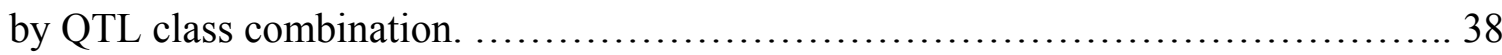

Figure 3.2: Mean yield (white bars) and DON levels (black bars) for wheat lines before selection for these traits (absence of selection), after the top high yielding 25\% lines were selected and after a subsequent top $13 \%$ of the lines was selected for low DON.

Figure 5.1: Regression of percentage of Fusarium damaged kernels measured by air separation (FDK) on percentage of Fusarium damaged kernels measured with NIR (NIRFDK) for all populations in Lexington 2010 and 2011.

Figure 5.2: Regression of deoxynivalenol level measured by traditional methods (DON) on deoxynivalenol level measured with NIR (NIRDON) on for all populations in Lexington 2010 and 2011.

Figure 5.3: Regression of deoxynivalenol level (DON) on percentage of Fusarium damaged kernels measured with NIR (NIRFDK) for all populations in Lexington 2010 and 2011.

Figure 5.4: Regression of deoxynivalenol level (DON) on percentage of Fusarium damaged kernels (FDK) for all populations in Lexington 2010 and 2011. 


\title{
Chapter One
}

\author{
Introduction
}

Fusarium Head Blight (FHB), or head scab is caused by Fusarium graminearum Schwabe (teleomorph Gibberella zeae (Schwein.) Petch; synonym = G. saubinetti) both in wheat (Triticum aestivum L. and T. durum L.) and barley (Hordeum vulgare L.). Although Fusarium graminearum is the principal causal agent in the US, many Fusarium species can cause FHB. Fusarium culmorum and Fusarium poae are prevalent in European countries (Bai and Shaner, 2004). Yield and test weight (TWT) reduction, contamination with the mycotoxin deoxynivalenol (DON), and additional costs of cleaning seed to improve grain quality have caused high economic losses for farmers and the industry (McMullen et al., 2008; McMullen et al., 1997). Losses in the Southeastern US in 2003 were estimated over \$ 13.6 million for 40 counties in Maryland, Virginia and North Carolina (Cowger and Sutton, 2005).

The greatest challenge in breeding for FHB resistance is to release adapted cultivars that combine competitive yield, acceptable end use quality, resistance to FHB and resistance to other diseases (Bai and Shaner, 2004; Buerstmayr et al., 2009). The quantitative nature of the inheritance of FHB resistance, its frequent association with undesirable agronomic traits and the large effect of the environment make breeding for this trait a very difficult task (Bai and Shaner, 2004). In addition, screening for scab resistance is time consuming and expensive. Molecular markers can be used to complement phenotyping and classical breeding to select for major resistance quantitative trait loci (QTL) (Agostinelli et al., 2012; Kang et al., 2011; Buersrmayr et al., 2002; Buerstmayr et al., 2009). QTL for FHB resistance were reported on almost all wheat chromosomes (Buerstmayr et al., 2010). Marker assisted selection (MAS) combines both phenotypic and QTL information and it assumes that not all of the QTL for the trait of interest are known (Bernardo, 2002). Markers representing the QTL or linked to QTL have to be validated in different genetic backgrounds for breeders to use them in their programs (Van Sanford et al., 2001). Chinese spring wheat cultivar Sumai-3 is the most widely used source of FHB resistance (Bai and Shaner, 1994; Rudd et al., 2001). QTL 
analysis of Sumai-3 and other Asian cultivars shows that almost all of them have a QTL for resistance on chromosome 3BS at the Qfhs.ndsu-3BS locus (Sneller et al., 2010) also known as Fhb1. VA01W-476, derived from resistant cultivars Roane and W14, combines two exotic QTL Fhb1 and QFhs.nau-2DL, with additional resistance (Agostinelli et al., 2012).

The kernel traits most indicative of head scab damage are Fusarium damaged kernels (FDK) and DON. Common methods to measure FDK involve quantifying the proportion of unhealthy grains in a random sample of grain. Both DON and FDK are expensive and time consuming to quantify; thus, rapid and non-destructive methods for predicting these traits are of great interest. Near-infrared reflectance detects the absorption response by overtone and combination frequencies of $\mathrm{O}-\mathrm{H}, \mathrm{C}-\mathrm{H}$, and $\mathrm{N}-\mathrm{H}$ molecular vibrations (Approved Method 39-00.01, 39-25.01, AACC, 2011) as well as physical properties like grain shape, size and color (Peiris et al, 2010) and is currently used to measure whole grain hardness, whole grain protein and flour protein. NIR has also shown good correlations with FDK and DON measured by traditional methods.

Soft red winter wheat is mainly used for cakes, cookies, crackers, donuts and flat breads (Beuerlein, 2001). Souza et al. (2012) proposed that selection to maintaining a quality type could be more efficient by focusing on a limited number of traits with large heritability values such as flour yield, softness equivalent, and solvent retention capacity (SRC). The SRC tests predict baking performance by measuring the weight of solvent (water, lactic acid, sucrose or sodium carbonate) retained as a percentage of the flour weight (Smith et al., 2011). Wheat meal-based assays that require small amounts of sample have been shown to be correlated with flour-based assays (Gutierri et al., 2004, Knott et al., 2009) and would be suitable for assessing quality in earlier generations.

The success of using exotic quantitative trait loci (QTL) for breeding wheat cultivars resistant to FHB depends on 1) effectiveness of the QTL in diverse genetic backgrounds, and 2) the effects of the QTL on agronomic and quality traits. This study was conducted to validate $F h b 1$ and $Q F h s . n a u-2 D L$ in terms of FHB, agronomic and milling and baking quality traits in different genetic backgrounds, to determine the utility NIR to measure FDK and DON, and the ability of NIR and of wheat meal assays to predict milling and baking quality. 


\section{Chapter Two}

Literature review

\section{Economic impact of Fusarium Head Blight}

Fusarium Head Blight (FHB), or head scab, is a fungal disease caused by Fusarium graminearum Schwabe (teleomorph Gibberella zeae (Schwein.) Petch; synonym $=G$. saubinetti) both in wheat (Triticum aestivum L. and T. durum L.) and barley (Hordeum vulgare L.). The same pathogen is the causal agent associated with stalk rot of corn (Zea mays L.). Even though Fusarium graminearum is predominant in the US, other species of Fusarium can cause head scab such as Fusarium culmorum and Fusarium Poae, which are more frequent in Europe (Bai and Shaner, 2004). Economic losses caused by FHB are associated with (i) yield reduction, (ii) lower price received as a consequence of poor test weight (TWT), contamination with the mycotoxin deoxynivalenol (DON), and additional costs of cleaning seed to improve grain quality (McMullen et al., 2008; McMullen et al., 1997).

In the United States (US), FHB outbreaks during the 90's led to a loss of \$ 4.8 billion (Johnson et al, 2003). Nganje et al. (2002) estimated the cumulative direct and secondary economic losses from FHB in hard red spring (HRS) wheat, soft red winter (SRW) wheat, durum wheat, and barley at \$ 2.7 billion from 1998 through 2000 . These authors point out that for every dollar in direct scab losses to farmers, more than two dollars in secondary economic effects are incurred. Losses due to FHB in the Southeastern US in 2003 had no precedents, estimated over \$ 13.6 million for 40 counties in Maryland, Virginia and North Carolina (Cowger and Sutton, 2005). More recently, in 2009, economic losses associated to FHB were estimated at \$ 30 million in Kentucky (Van Sanford, personal communication, 2012)

Infected florets may fail to produce grains leading to a lower number of grains per unit area; thus, reducing yield. When infected florets do produce grains, kernels are often shriveled, lighter and discolored, and are commonly known as scabby kernels or tombstones (Bai and Shaner, 2004). These kernels present a floury discolored interior (Ruckenbauer et al., 2001). The presence of Fusarium damaged kernels (FDK) reduces 
TWT and increases the levels of DON in grain samples. Moreover, if tombstones are light enough, they are expelled from the combine during the harvest process contributing to yield reduction. In practice, allowing more air flow during harvest is a common way to reduce the number of tombstones in order to reduce DON. The pathogen may affect both the physical and the physiological aspects of seed quality, including seed size, composition, germination, and vigor (Argyris et al., 2001). Impact on animal production and seed quality are considered among indirect losses (Bai and Shaner, 1994; McMullen et al., 1997).

Current Food and Drug Administration's (FDA) advisory levels for DON are: $1 \mathrm{ppm}$ on finished wheat products that may potentially be consumed by humans, and 5-10 ppm on grains and by-products for animal consumption, depending on the animal species and the proportion in their diet (FDA, 2010). In the EU, maximum DON content allowed is $1.25 \mathrm{ppm}$ for unprocessed wheat and 0.5 in bread and baked goods (Anonymous, 2005)

\section{Pathology and epidemiology of Fusarium graminearum}

Although several species of Fusarium can cause FHB, F. graminearum is the principal causal agent (Gale, 2003; Bai and Shaner 1994). Warm and humid weather conditions are required for fungal infection (Dufault et al., 2002) with a moist period of 36-72 hours. Optimum temperature was reported to be 25 C (Bai and Shaner, 1994). Dufault et al. (2002) evaluated perithicia production on crop residue under controlled temperature and humidity conditions and found that the number of perithicia produced at 15 or $25^{\circ} \mathrm{C}$ was greater than at $30^{\circ} \mathrm{C}$, but no significant difference was detected between 15 and $25^{\circ} \mathrm{C}$. High moisture levels presented larger amounts of perithicia production. Wheat plants are most susceptible during anthesis but infection can occur any time after the beginning of flowering (Bai and Shaner, 2004; Stack, 1999)

$F$. graminearum survives on alternative hosts such us corn, barley, soybeans and rice, and saprophytically, in dead tissue (Bai and Shaner, 2004). Macroconidia, mycelia and clamydospores are formed by the asexual stage and ascospores by the sexual stage (Bai and Shaner, 1994). Crop residues are the most important source of primary inoculum in the world (Bai and Shaner, 1994). In Kentucky, the pathogen overwinters primarily in corn stubble and under favorable conditions it produces perithicia that release ascospores 
which are blown into fields from remote or local sources and/or are splashed onto nearby heads (Hershman and Johnson, 2009). Ascospores germinate on or inside spikelets and infection begins. The pathogen colonizes extruded anthers and grows throughout the developing reproductive structures. It may also directly penetrate the glume, palea or rachilla (Bai and Shaner, 1994).

Symptoms appear shortly after infection as dark brown water-soaked spots on glumes. Infected spikelets and adjacent spikelets appear blighted if vascular tissue is clogged by mycelium (Bai and Shaner, 1994). The proportion of bleached spikelets and green spikelets are diagnostic for the disease. The disease can also be diagnosed by the presence of pink to salmon-orange spore masses on spikelets and glumes (McMullen et al., 2008).

Mesterhazy et al. (1999) conducted a set of experiments inoculating a range of genotypes representing resistance levels in Europe. Wheat lines were inoculated with isolates of $F$. graminearum and $F$. culmorum collected in different European countries. Isolates were used independently, not mixed, to evaluate host-pathogen specificity. The authors found that in cases, some isolates had a negligible preference for some genotypes, but with no practical significance from a breeding perspective. In agreement with studies

conducted in the US with $F$. graminearum (Bai and Shaner, 1996), the authors concluded that there is not a race-specific pattern in the two species evaluated.

\section{Host resistance to Fusarium Head Blight: mechanisms and genetic sources of resistance}

Host resistance is the most important method to control FHB (Sneller et al., 2010; Rudd et al., 2001; McMullen et al., 1997). The inheritance of FHB resistance is quantitative in nature and it comprises multiple traits controlled by several major and minor quantitative trait loci (QTL) (Bai and Shaner, 2004; Buerstmayr, 2002; Buerstmayr et al., 2009). Although additive effects account for most of the genetic variance, dominance and epistasis have been reported as well (Bai et al., 2000). Based on earlier findings and the results of a set of experiments conducted to study the nature of the resistance to FHB in wheat, Mesterhazy et al. (1999) summarized five components: (i) 
resistance to invasion or penetration (Type 1), (ii) resistance to spreading (Type 2), (iii) resistance to toxin accumulation, (iv) resistance to kernel infection, and (v) tolerance.

Frequent epidemics in Asia led to a long tradition of breeding for FHB resistance in that part of the world (Buerstmayr et al., 2009). Sumai-3, a Chinese spring wheat cultivar, is the most widely used source of FHB resistance (Bai and Shaner, 2004; Rudd et al., 2001). However, Rudd et al. (2001) stated that breeders should be cautious about the use of Sumai-3 because there are different selections that differ both for FHB resistance and agronomic traits. QTL analysis of Sumai-3 and other Asian cultivars show that almost all of them have a QTL for resistance on chromosome 3BS at the Qfhs.ndsu$3 B S$ locus (Sneller et al., 2010) also known as Fhbl.

Bai and Shaner (2004) reviewed the different available sources of FHB resistance. In their article, they mention Japanese accessions such us Shinchunaga, Nobeokabouzu and Nyu Bai. Although these are resistant to FHB, they are inferior to Sumai-3 for agronomic traits. Frontana and Encruzilhada, from Brazil, are also mentioned in this review for their low incidence of FHB in field conditions. Frontana is thought to carry moderate type 1 resistance mainly based on morphological traits like hard glumes and narrow flower opening (Buerstmayr et al., 2009).

In Europe, several winter wheat cultivars have been used as sources of resistance to FHB. Sincron, from Romania, and Arina, from Switzerland, are mentioned by Buerstmayr et al. (2009) in a recent review.

In the US, Ernie (McKendry et al., 1995), Freedom (Gooding et al., 1997) and Roane (Griffey et al., 2001) were reported to have low FHB index in the field. Truman (McKendry et al., 2005), released by the University of Missouri, has a better combination of both FHB resistance and desirable agronomic traits. VA01W-476, derived from resistant cultivars Roane and W14, combines two exotic QTL Fhb1 and QFhs.nau-2DL, with additional resistance (Agostinelli et al., 2012).

Although additional sources of resistance were found among alien species in China and Japan, such resistance is not superior to that of wheat species and is often associated with undesirable traits (Bai and Shaner, 2004). However, Buerstmayr et al. (2009) pointed out that because in tetraploid durum wheat (T. durum), most cultivars are 
susceptible to FHB and there is low variability for this trait, wild or cultivated relatives should provide alternative sources of resistance.

\section{Breeding for Fusarium Head Blight resistance: conventional breeding and marker assisted selection}

Releasing adapted cultivars combining good agronomic performance, acceptable end use quality, resistance to FHB and other diseases is a challenge for plant breeders (Bai and Shaner, 2004; Buerstmayr et al., 2009). The quantitative nature of the inheritance of FHB resistance, its frequent association with undesirable agronomic traits and the large effect of the environment make breeding for this trait very difficult (Bai and Shaner, 2004). Moreover, screening for FHB resistance is time consuming and expensive. Molecular markers can be used to assist phenotyping and complement classical breeding to select for major resistance QTL (Agostinelli et al., 2012; Kang et al., 2011;

Buersrmayr et al., 2002; Buerstmayr et al., 2009)

Breeding programs selecting for scab resistance utilize three basic strategies: (i) improvement of agronomic traits in highly resistant germplasm, (ii) improvement of resistance in released cultivars, and (iii) introduction of new resistance genes from other gene pools (Bai and Shaner, 2004). Pedigree method, single seed descent and recurrent selection are used in conventional breeding for FHB resistance (Rudd et al., 2001). Yang et al. (2000) reported an average decrease in diseased spikelets of $10 \%$ per cycle after four cycles of recurrent selection using the dominant male sterile gene $m s_{2}$ in spring wheat.

Sneller et al. (2010) deduced the genetics of FHB resistance in eastern US SRW wheat using heritability estimates, population means and frequency of different reaction types and transgressive segregants. 2983 lines from 223 parents were evaluated in single and multiyear trials in inoculated scab nurseries. Without selection, the authors found $41 \%$ of the lines with moderate resistance (Freedom used as a reference) and $70 \%$ of the crosses showed segregation for the FHB trait evaluated. They conclude that the observed levels of resistance could only be explained by a high frequency of resistance contributing alleles in the population under study. Entry-mean heritability estimates 
ranged from 0.35 to 0.89 . The authors suggest recurrent selection in this population should provide large gains in scab resistance without the introduction of exotic QTL.

QTL for FHB resistance were reported in almost all wheat chromosomes (Buerstmayr et al., 2010). In a recent review, Buerstmayr et al. (2009) reported 22 FHB resistance QTL regions detected in more than one mapping population: 1B (two regions), 1D, 2A (2), 2B (2), 2D (2), 3A, 3B (2), 3D, 4B, 4D, 5A, 5B, 6A, 6B, 7A and 7B (2). Most repeatable QTL are those based on Asian genetic resources: Fhbl (Chromosome 3BS), Qfhs.ifa-5A (5A) and Fhb2 (Chromosome 6BS) (Buerstmayr et al., 2010). Marker assisted selection (MAS) combines both phenotypic and QTL information and it assumes that not all of the QTL for the trait of interest are known (Bernardo, 2002). Markers representing the QTL or linked to QTL have to be validated in different genetic backgrounds for breeders to use them in their programs (Van Sanford et al., 2001)

Fhbl has been reported to explain $29 \%$ of the phenotypic variance in severity and to be associated with fungal spread within the spike (Type 2 resistance) in a $364 \mathrm{~F}_{1}$ derived doubled-haploid spring wheat lines from a resistant (carrying Sumai-3 resistance) and a susceptible parent. In the same study, the QTL on chromosome 5A explained 20\% of the variation in the same trait and was more associated to resistance to fungal penetration (Type 1 resistance) (Buerstmayr et al., 2003). Pumphrey et al. (2007) estimated 27\% reductions in FDK associated with Fhbl using spring wheat near-isogenic lines (NIL).

In a SRW wheat population derived from a 2-way cross of a high yielding susceptible parent and a resistant parent, Agostinelli et al. (2012) found that Fhbl reduced FDK and DON by 32 and 25\%, respectively. In this genetic background, QFhs.nau-2DL had a more pronounced effect: 40 and 55\% reduction in FDK and DON, respectively. Both QTL complemented one another and when combined, they reduced FHB levels more than each QTL alone. When they compared simulated phenotypic and genotypic selection, based on both QTL, they found similar effectiveness in reducing FHB. Based on their findings, the authors propose an initial round of phenotypic selection (moderate intensity, 25-35\%) to enrich the population with major resistance QTL alleles and to ensure variation at both minor resistance loci and other traits such as yield and quality. 
Kang et al. (2011) studied the effects of Fhb1, QFhs.nau-2DL and a QTL on chromosome 5A on eight SRW wheat NIL developed by marker assisted backcrossing in Maryland and Kentucky. The NIL were derived from a resistant non-adapted cultivar Ning7840 (donor) and the adapted SRW wheat McCormick. In this background, the combination of $F h b 1$ and $Q F h s . n a u-2 D L$ resulted in more resistant lines and lower levels of DON. This combination was not different from having the three QTL together. The authors concluded that $F h b 1$ and $Q F h s$.nau-2DL together would be useful in breeding for FHB resistance in the mid-Atlantic region.

Marker-assisted backcrossing was proposed by Buerstmayr et al. (2002) to obtain lines with desirable agronomic performance and QTL for resistance to FHB. One year of backcrossing to a high yielding recurrent parent was enough to restore this trait in SRW wheat lines with resistant QTL Fhb1 and QFhs.nau-2DL (Daniela Sarti, personal communication, 2012).

Von der Ohe et al. (2010) investigated the effects of the introgression of Fhbl and a QTL on chromosome $5 \mathrm{~A}$ on agronomic and baking quality traits in two $\mathrm{BC}_{3} \mathrm{~F}_{2: 5}$ winter wheat populations. Although both QTL improved disease resistance, the magnitude of the improvement depended on the level of the resistance of the recurrent parent. Overall, they authors found small negative effects on agronomic traits such as $5 \%$ yield reductions in one of the populations when comparing lines carrying both QTL and susceptible lines. Other than a slight increment in TWT due to the QTL on chromosome 5A, QTL effects were not significant for TWT, SDS sedimentation (SDS) nor grain protein concentration. The authors concluded that minor negative effects can be minimized by selecting high grain yield and $\mathrm{FHB}$ resistance within the $\mathrm{BC}_{3}$ lines. In this study, resistance due to QTL effects was not associated with plant height or lateness. McCartney et al. (2007) conducted a similar study using three $\mathrm{BC}_{2}$ populations of Canadian spring wheat. The QTL studied were Fhb1, QTL on chromosomes 3BSc, 4B, 2D and 5AS. In this genetic background, the QTL resistant allele on chromosome 4B presented the largest impact in reducing FHB but it showed a strong correlation between increased plant height and improved FHB resistance. No relationship with lateness was found. The presence of the QTL on chromosome 2D increased TWT and grain protein concentration. The QTL resistant alleles on chromosome 3BSc were also associated with increased TWT. The 
QTL on chromosome 5AS reduced thousand grain weight and grain protein in one of the populations. Fusarium Head Blight traits were negatively correlated with plant height. Recombinant inbred lines derived from Ning7840 (Chinese hard red spring wheat) and Pioneer 2643 (SRW wheat) carrying susceptible and resistant versions of Fhb1, a QTL on chromosome 5A and QFhs.nau-2DL were studied to analyze the impact of these QTL on FHB and to identify linkage drags on agronomic and quality traits (Cardwell, 2011). Fhbl was associated with FHB resistance and no detrimental effects on agronomic, milling and baking traits. On the other hand, QFhs.nau-2DL and the QTL on chromosome $5 \mathrm{~A}$ reduced kernel weight, milling quality score, softness equivalence, flour yield, flour protein, and lactic acid solvent retention capacity (SRC). The QTL on chromosome 5A was also associated with lodging and lower TWT.

\section{Soft red winter wheat quality}

Soft red winter wheat is mainly used for cakes, cookies, crackers, donuts and flat breads (Beuerlein, 2001). Quality refers to the specific composition and rheological functionality of flours and those requirements vary depending on the intended use. Baking quality is mainly a function of gluten strength and water absorption. Milling quality is the result of flour yield and flour particle size (Smith et al., 2011). Gluten strength is a function of protein concentration and protein composition (glutenin:gliadin) (Guttieri et al., 2001; Smith et al., 2011). Crackers and flatbreads require higher levels of gluten strength than cookies (Gutierri et al., 2001).

The flour solvent retention capacity (SRC) tests predict baking performance by measuring the weight of solvent retained as a percentage of the flour weight (Smith et al., 2011). Solvents used are 5\% w/w sodium carbonate, $50 \% \mathrm{w} / \mathrm{w}$ sucrose and $5 \%$ lactic acid, that are used to predict the functional contribution of starch, pentosans and glutenins, and a combination of these three components, respectively (Kweon et al., 2011). All of these components contribute to $P_{\max }$ (peak of pressure) in a typical alveograph profile (Kweon et al., 2011). For this reason, the different SRC tests provide more information about flour quality and help predict functionality of flours that may have the same alveogram parameters but different flour composition. The principle underlying the SRC tests is that cross-linked polymeric materials swell in the presence of 
solvents and that swelling can be measured by changes in weight (Kweon et al., 2011). Flour water SRC measures global water affinity of starch, arabinoxylans, gluten, and gliadins. This parameter is negatively correlated to flour yield and softness equivalent (Souza et al., 2011). Cookies and crackers made with flours with very high water retention require longer cooking time. This results in less tender and cost efficient products (Guttieri et al., 2001). Flour sucrose SRC is a measure of arabinoxylans content that affect water absorption in baked products. It is considered the best predictor of cookie quality. There is a negative correlation between flour sucrose SRC and with wirecut cookie diameter $(\mathrm{r}=-0.66)$, flour yield $(\mathrm{r}=-0.31)$ and softness equivalent $(\mathrm{r}=-0.23)$. Flour sodium carbonate SRC measures starch damage. The solvent used in this test ionizes the ends of starch polymers increasing its water binding capacity. Flour sodium carbonate SRC predicts flour yield $(\mathrm{r}=-0.48)$ and cookie diameter $(\mathrm{r}=-0.22)$. Flour lactic acid SRC predicts gluten strength and is correlated with the SDS test and flour protein concentration (Souza et al., 2011). The SDS test is used to select bread quality in hard wheat and the wheat meal (WM) based SDS has been proposed to identify lines with higher gluten strength in soft wheat (Knott et al., 2009). Given the functional properties each test predicts, the desired directions for flour water SRC, flour sodium carbonate SRC and flour sucrose SRC is towards lower values, whereas for flour lactic acid SRC is towards larger values. Gold standard targets published by Kweon et al. (2011) for cookies and crackers are $<=51$ flour water SRC, $<=64$ flour sodium carbonate $\mathrm{SRC},<=89$ flour sucrose SRC and $>=87$ flour lactic acid SRC. For sponge-and-dough products the targets are $<=57$ WSRC,$<=72$ SCSRC,$<=96$ SSRC and $>=100$ LASRC. These authors suggest using the gluten performance index (GPI) that is the ratio between flour lactic acid $\mathrm{SRC} /($ flour sodium carbonate $\mathrm{SRC}+$ flour sucrose $\mathrm{SRC}$ ) as an overall performance predictor.

Knott et al. (2009) evaluated WM assays for their ability to select lines with acceptable soft wheat quality in early generations. The advantages of WM assays are the small amount of grain required and that sample mills are relatively inexpensive. A previous study (Gutierri et al., 2004) showed a very good prediction ability of flour sodium carbonate SRC and flour sucrose SRC by WM-sodium carbonate SRC (r=0.690.81 and $r=0.74-0.84$, respectively). The ability of predicting both flour SRC with only 
WM-sodium carbonate SRC test is beneficial both in terms of time and resources because the sodium carbonate solvent is much less concentrated than the sucrose solvent. Wheat meal SDS in their study show an excellent ability to predict flour lactic acid SRC $(\mathrm{r}=0.74-0.93)$. Wheat meal SDS test presents advantages as compared to both flour and WM-lactic acid SRC because it requires only 1 gram of wheat meal and the laboratory procedure is less time consuming. Knott et al. (2009) estimated broad-sense heritability for WM-sodium carbonate SRC and WM-SDS to be 0.70 and 0.67 , respectively. Wheat meal SDS was correlated to flour protein $(\mathrm{r}=0.29)$, flour lactic acid SRC $(\mathrm{r}=0.37)$ and wire-cut cookie measures $(\mathrm{r}=-0.42)$. Wheat meal sodium carbonate SRC was correlated to flour yield ( $\mathrm{r}=-0.40)$, flour sucrose SRC $(\mathrm{r}=-0.37)$, flour sodium carbonate $\mathrm{SRC}(\mathrm{r}=0.46)$, flour water SRC $(r=0.47)$ and wire-cut cookie diameter $(r=-0.33)$. The researchers concluded that WM assays could be an easy and economical alternative to increase the proportion of experimental lines with desirable milling and baking quality in breeding programs.

To determine the basis for selection of soft wheat for end-use quality, Souza et al. (2012) conducted a multi-loc study during 2006 and 2007. Representative cultivars grown in eastern North America and covering a range of milling and baking quality performance were tested in yield plot trials. The authors found small $G^{*} E$ for most quality traits, except for TWT, and stated that selection to maintaining a quality type could be more efficient by focusing on a limited number of traits with large heritability values such as flour yield, softness equivalent, and SRC.

\section{Use of near infrared reflectance to measure FHB and quality traits}

Near infrared (NIR) reflectance is of great interest for measuring FDK, DON and quality parameters for its simplicity, time use efficiency and objectivity. If performed on whole grain samples, this method is of even more interest for breeders because it is nondestructive. As described in the AACC Approved Methods guidelines, the absorption response by overtone and combination frequencies of $\mathrm{O}-\mathrm{H}, \mathrm{C}-\mathrm{H}$, and $\mathrm{N}-\mathrm{H}$ molecular vibrations is discernible by photometric detectors, which inherently have a very high signal-to-noise response. Thus, the composition (protein, carbohydrate, moisture, lipid and others) of cereals can be determined (Approved Method 39-00.01, 39-25.01, AACC, 
2011). For example, protein content in whole grain wheat samples is based on transmittance or reflectance of near-infrared (850-2,500 nm) energy (Approved Method 39-25.01, AACC, 2011). Physical properties, such us grain shape, size and color in FDK, also impact the NIR absorption levels (Peiris et al., 2010).

Delwiche and Hareland (2004) found an accuracy of $95 \%$ in the detection of FDK using NIR (1,000 -1,700 nm) on an individual kernel basis. A subsequent study showed that the spectral absorption near 1,200 nm, attributed to ergosterol (a primary constituent in fungi cell membranes), useful in spectral recognition of FDK (Delwiche et al., 2011). In this study, the authors combined NIR with hyperspectral imaging (visible spectra, 400$1,000 \mathrm{~nm}$ ) to be able to detect lower levels of DON, more difficult to detect on the basis of NIR only. Peiris et al. (2010) evaluated single kernel NIR (SKNIR) to measure FDK and predict DON levels. The authors found that kernels could be separated in sound and scabby fractions. In high DON single kernels, the level of DON was predicted with a coefficient of determination $\mathrm{R}^{2}=0.87$.

The ability of NIR to predict DON levels in both 125 grams whole grain and milled samples was tested against traditional chromatographic methods in the Southern Brazil. The authors reported 0.89 and $0.91 \mathrm{R}^{2}$, respectively (Tibola, C.S).

A calibration built in 2007 by the University of Kentucky and Perten Instruments resulted in Coefficients of determination $\left(\mathrm{R}^{2}\right)$ of FDK measured using NIR, and FDK and DON measured by traditional methods, of 0.67 and 0.59 , respectively. (Agostinelli, unpublished data). 


\section{Chapter Three}

Effects of Fhbl and QFhs.nau-2DL on FHB, agronomic and quality traits

\section{Introduction}

In the US, Fusarium Head Blight (FHB), or head scab is primarily caused by Fusarium graminearum Schwabe (teleomorph Gibberella zeae (Schwein.) Petch; synonym $=$ G. saubinetti) both in wheat (Triticum aestivum L. and T. durum L.) and barley (Hordeum vulgare L.). Yield and test weight (TWT) reduction, contamination with the mycotoxin deoxynivalenol (DON), and additional costs of cleaning seed to improve grain quality have caused severe economic losses for farmers and the industry (McMullen et al., 2008; McMullen et al., 1997). Losses in the Southeastern US in 2003 were estimated over \$ 13.6 million for 40 counties in Maryland, Virginia and North Carolina (Cowger and Sutton, 2005).

The use of exotic resistance quantitative trait loci (QTL) provides one strategy for breeding wheat cultivars resistant to Fusarium Head Blight (FHB). The success of this approach depends on 1) effectiveness of the QTL in diverse genetic backgrounds, and 2) the effects of the QTL on agronomic and quality traits. More robust information on the effects of Fhbl and QFhs.nau-2DL in multiple SRW wheat genetic backgrounds is required. The quantitative nature of FHB resistance, its frequent association with undesirable agronomic traits and the large effect of the environment make breeding for this trait very difficult (Bai and Shaner, 2004). In addition, screening for scab resistance is time consuming and expensive. Molecular markers can be used to complement phenotyping and classical breeding to select for major resistance QTL (Agostinelli et al., 2012; Kang et al., 2011; Buersrmayr et al., 2002; Buerstmayr et al., 2009). QTL for FHB resistance have been reported on almost all wheat chromosomes (Buerstmayr et al., 2010). Marker assisted selection (MAS) combines both phenotypic and QTL information and it assumes that not all of the QTL for the trait of interest are known (Bernardo, 2002). Markers representing the QTL or linked to the QTL have to be validated in different genetic backgrounds for breeders to use them in their programs (Van Sanford et al., 
2001). The Chinese spring wheat cultivar Sumai-3 is the most widely used source of FHB resistance (Bai and Shaner, 1994; Rudd et al., 2001). QTL analysis of Sumai-3 and other Asian cultivars shows that almost all of them have a QTL for resistance on chromosome 3BS at the Qfhs.ndsu-3BS locus (Sneller et al., 2010) also known as Fhb1. VA01W-476, derived from resistant cultivars Roane and W14, combines two exotic QTL, Fhb1 and QFhs.nau-2DL, with additional resistance (Agostinelli et al., 2012).

Fhb1 has been reported to explain $29 \%$ of the phenotypic variance in severity and to be associated with fungal spread within the spike (Type 2 resistance) in $\mathrm{F}_{1}$ derived doubled-haploid spring wheat lines from a resistant (carrying Sumai-3 resistance) and a susceptible parent (Buerstmayr et al., 2003). Pumphrey et al. (2007) estimated 27\% reductions in FDK associated with Fhbl in near-isogenic spring wheat lines. In a SRW wheat population derived from a single cross of a high yielding susceptible parent and a resistant parent, Agostinelli et al. (2012) found that Fhb1 reduced FDK and DON by 32 and $25 \%$, respectively. The authors also evaluated QFhs.nau-2DL and they found 40 and $55 \%$ reduction in FDK and DON, respectively. When combined, both QTL complemented one another reducing FDK and DON more than each individual QTL. Kang et al. (2011) studied the effects of Fhb1, QFhs.nau-2DL and a QTL on chromosome 5A on eight SRW wheat near-isogenic lines (NIL) developed by marker assisted backcrossing in Maryland and Kentucky. The combination of Fhbl and QFhs.nau-2DL resulted in more resistant lines and lower levels of DON, and it was not different from having the 3 QTL together. The researchers concluded that Fhbl and QFhs.nau-2DL together would be useful in breeding for FHB resistance in the midAtlantic region.

The greatest challenge in breeding for FHB resistance is to release adapted FHB resistant cultivars that combine competitive yield and acceptable end-use quality (Bai and Shaner, 2004; Buerstmayr et al., 2009). SRW wheat is mainly used for cakes, cookies, crackers, donuts and flat breads (Beuerlein, 2001). Traits used to characterize soft wheat quality include flour yield, flour protein, softness equivalent, and flour solvent retention capacity (SRC). The SRC tests predict baking performance by measuring the weight of solvent (water, lactic acid, sucrose or sodium carbonate) retained as a percentage of the flour weight (Smith et al., 2011). In a recent review, Kweon et al. (2011) suggest using 
the gluten performance index (GPI), that is the ratio between flour lactic acid SRC/(flour sodium SRC + flour sucrose SRC), as an overall performance predictor. Taking minimum gold standard targets published in this review, the GPI would be 57\%. A higher percentage given by lower flour sucrose or sodium carbonate, or higher flour lactic acid $\mathrm{SRC}$, would be better for baking cookies.

The objectives of this study were to evaluate the impact of Fhbl and QFhs.nau$2 D L$ on (i) FHB traits, agronomic traits and milling and baking quality in five different SRW wheat populations, (ii) to simulate progress from direct (DON) and indirect (FDK, NIRFDK, FHB index) phenotypic selection and genotypic selection using data from two years, and (iii) to assess the utility of NIR for estimating FDK and DON.

\section{Materials and methods}

\section{Plant material}

Five sets of inbred lines derived from 2 and 3-way crosses were evaluated in this study: 1) 26R58/VA01W-476//KY97C-0574-01, 2) 25R54/VA01W-476//KY97C-057401, 3) 25R54/VA01W-476//KY97C-0554-02, 4) 25R78/VA01W-476 and 5) KY93C1238-17-1/VA01W-476. Crosses were made between FHB susceptible parents and FHBresistant VA01W-476, a doubled haploid line derived from the cross 'Roane'/W14. These crosses represent typical resistant x susceptible crosses used in SRW wheat breeding programs. Lines comprising each population were chosen from an initial group of 27 crosses genotyped at the Regional Small Grains Genotyping lab (RSGGL) in Raleigh, NC (http://www.ars.usda.gov/saa/psru, verified 2/22/11) in 2007. $\mathrm{F}_{2}$ progeny were evaluated for the presence of resistance alleles at Fhbl and QFhs.nau-2DL. Markers used were Xgwm533 (Röder et al., 1998) for Fhb1, and Xcfd233 (Grain genes 2.0 at http://wheat.pw.usda.gov/GG2/index.shtml, verified 02/13/09) for QFhs.nau-2DL. Ten heads from each $\mathrm{F}_{2: 3}$ head row were threshed in bulk in 2008 to provide seed for this study.

To evaluate FHB traits, $\mathrm{F}_{2: 5}$ and $\mathrm{F}_{2: 6}$ lines were planted in headrows in a scab nursery at Spindletop Research Farm (38'37.81', N, 84²9'44.85'’W; Maury silt loam [fine, mixed, semiactive, mesic Typic Paleudalfs]) near Lexington, KY (LEX). The populations were also grown in yield trials at LEX (2010 and 2011) and Princeton 
(PRN2011), KY at the West Kentucky Research and Educational Center (376 $7.37^{\prime \prime}$ N, 8752'13.62' W; Crider silt loam [fine-silty, mixed, active, mesic Typic Paleudalfs]), to measure agronomic traits.

\section{Lexington scab nursery}

Two replicates of each line were sown in a randomized complete block (RCB) experiment in the 2009 Lexington scab nursery for FHB evaluation and bulked for planting in 2010. Lines were planted in rows $1.2 \mathrm{~m}$ long, spaced $30 \mathrm{~cm}$ apart. The scab nursery was planted on 12 October 2009 and 20 October 2010. To provide favorable conditions for the disease, rows were misted with an overhead mist irrigation system on an automatic timer. Mist irrigation was on from May 11 to June 16, for periods of 5 minutes, every quarter hour from $8 \mathrm{pm}$ to $8: 45,11 \mathrm{pm}$ to $11: 45 \mathrm{pm}, 2: 00$ to $2: 45 \mathrm{am}, 5$ to 5:30 am and 8.30 am (e.g. the equipment operated from $8 \mathrm{pm}$ to 8:05 pm the first time and the last time in the misting cycle was from 8:30 am to 8:35 am). Both years the nursery was inoculated with Fusarium graminearum- infected corn (Zea mays L.) (Verges et al., 2006). Inoculum comprised twenty-seven isolates taken from scabby wheat seed collected from 2007 to 2010 in multiple locations across Kentucky. For inoculum preparation, dry corn was set to imbibe water for $16 \mathrm{~h}$ before autoclaving. After autoclaving, the corn was inoculated with PDA plugs of Fusarium graminearum, mixed with $0.2 \mathrm{~g}$ streptomycin in $50 \mathrm{ml}$ sterile water, covered and incubated at room temperature for 3 weeks until it was fully colonized by the fungus. At this point, the corn was manually spread on a sterilized plastic sheet until dry, put in mesh bags and stored in the freezer until used. On 14 April the corn was spread between rows at a rate of 11.86 $\mathrm{gm}^{-2}$. Liquid nitrogen fertilizer (28\% UAN) was applied in the spring at a rate of $105 \mathrm{~kg}$ $\mathrm{N} /$ ha in split applications. Harmony Extra herbicide was applied on 20 April and a second application of Fusarium graminearum-infected corn was applied on 21 April.

\section{Yield plots}

The five populations were grown in six row plots $3 \mathrm{~m}$ long. The experimental design was a RCB with three replications, grown in LEX in 2010 and 2011 and PRN in 2011, planted on 12 October 2009, 20 October 2010 and 14 October 2010, respectively. 
All experimental plots received $105 \mathrm{~kg} \mathrm{~N} / \mathrm{ha}$ applied in the spring as described previously. Recommended agricultural practices for wheat production in Kentucky were followed (Lee et al., 2009).

\section{Phenotyping}

Heading date was recorded when $50 \%$ of the spikes in the row had emerged. Plant height was measured at the soft dough stage. FHB traits measured included incidence, severity, FHB index (severity * incidence), visual rating ( $0-9$, where 0 equals absence of FHB symptoms and 9 equals and FHB index $\geq 90 \%$ ), Fusarium damaged kernels (FDK) and deoxynivalenol (DON) concentration (ppm). Incidence was estimated by counting the number of blighted spikes in a random sample of 20 spikes in each row 24 days after heading. Severity was measured as the proportion of infected spikelets/total spikelets per spike, in 10 heads per row 24 days after heading. Samples were mechanically threshed and cleaned with low air flow to ensure minimal loss of scabby kernels. Approximately 45-g grain samples from each row were cleaned by hand and subsequently evaluated for FDK using an air separation machine specifically developed from a Precision Machine head thresher and a Shop-Vac vacuum to separate scabby kernels from healthy ones as described in (Agostinelli et al., 2012). FDK was expressed as the weight of scabby kernels divided by total weight. A 20-g sample in which scabby kernels had been recombined with sound kernels was analyzed for DON at University of Minnesota DON testing laboratory using gas chromatography with mass spectrometry (GC-MS) (Mirocha et al., 1998). To predict FDK and DON, samples were also run on a near-infrared analyzer (DA7200, 950-1650 nm wavelength range) manufactured by Perten Instruments (IL). A calibration built in 2007 by the University of Kentucky Wheat Breeding Program and the manufacturers showed strong positive correlations between FDK and DON measured with traditional methods and NIR in two-way crosses. Coefficients of determination $\left(\mathrm{R}^{2}\right)$ of FDK measured using NIR, and FDK and DON measured by traditional methods, were 0.67 and 0.59 , respectively (Agostinelli, unpublished data). This calibration was used to run 2010 samples and it was updated on the basis of 2010 FDK and DON data measured by air separation and GC-MS, respectively, to run 2011 samples. The amount of seed required is approximately $20 \mathrm{~g}$. 
Heading date, foliar disease ratings, FHB ratings and plant height measurements were taken in yield plots. Each plot was harvested with a mechanical combine for yield and TWT determination. A 100 gram sample from each rep was analyzed for milling and baking quality at the USDA-ARS Soft Wheat Quality Laboratory, Wooster, OH. All grain was tempered at $15 \%$ moisture before milling. Flour yield was calculated as the bran weight subtracted from the grain weight, divided by the grain weight times 100 as described in Souza et al. (2008). Softness equivalent was calculated from the fraction of mill product that is in the mids that is subtracted from the adjusted flour yield. Water SRC, sucrose SRC, sodium carbonate SRC, and lactic acid SRC were estimated using approved AACC Method 56-11.02 (AACC, 2010) and were used to calculate GPI as described by Kweon et al. (2011).

\section{Data analysis}

Analysis of variance (ANOVA) was performed using the General Linear Models procedure (Proc GLM; SAS 2002) to determine line and QTL effects. The model used was:

$\mathrm{Y}_{\mathrm{ij}}=\mu+\mathrm{ENV}_{\mathrm{i}}+\mathrm{R}(\mathrm{ENV})_{\mathrm{ij}}+\mathrm{QTL}+\mathrm{G}_{\mathrm{j}}(\mathrm{QTL})+\mathrm{ENV}_{\mathrm{i}} * \mathrm{G}_{\mathrm{j}}+\mathrm{E}_{\mathrm{ij}}$

Where: $\mathrm{Y}_{\mathrm{ij}}=$ the observation in the $k$ th genotype in the $j$ th rep in the $i$ th environment, $\mu=$ the overall mean, $\mathrm{G}_{\mathrm{j}}(\mathrm{QTL})=$ the effect of the $k$ th genotype within QTL, QTL $=$ the effect of the QTL, R(ENV) $)_{\mathrm{ij}}=$ the effect of $j$ th rep within $i$ th environment, $\mathrm{ENV}_{\mathrm{i}} * \mathrm{G}_{\mathrm{j}}=$ the effect of the interaction of the $i$ th environment with the $k$ th genotype, $\mathrm{E}_{\mathrm{ij}}=$ the residual error. Fisher's Least Significant Difference (LSD) was used to corroborate significamt differences among QTL combination classes.

Broad sense heritability of FHB and agronomic traits was estimated on an entry mean basis using the following model:

$\mathrm{Y}_{\mathrm{ij}}=\mu+\mathrm{G}_{\mathrm{k}}+\mathrm{R}(\mathrm{ENV})_{\mathrm{ij}}+\mathrm{ENV}_{\mathrm{i}} * \mathrm{G}_{\mathrm{j}}+\mathrm{E}_{\mathrm{ij}}$

Where: $\mathrm{Y}_{\mathrm{ij}}=$ the observation in the $k$ th genotype in the $j$ th rep in the $i$ th environment, $\mu=$ the overall mean, $\mathrm{G}_{\mathrm{j}}=$ the effect of the $k$ th genotype, $\mathrm{R}(\mathrm{ENV})_{\mathrm{ij}}=$ the effect of $j$ th rep within $i$ th environment, $\mathrm{ENV}_{\mathrm{i}} * \mathrm{G}_{\mathrm{j}}=$ the effect of the interaction of the $i$ th environment with the $k$ th genotype, $\mathrm{E}_{\mathrm{ij}}=$ the residual error. 
Data was analyzed using the General Linear Models procedure (Proc GLM; SAS 2002). Genotypic and phenotypic variances were estimated from the expected mean squares (EMS) and heritability estimates were computed as:

$\mathrm{h}^{2}=\mathrm{V}_{\mathrm{g}} / \mathrm{V}_{\mathrm{p}}$

where $\mathrm{h}^{2}=$ heritability, $\mathrm{V}_{\mathrm{g}}=$ genotypic variance, $\mathrm{V}_{\mathrm{p}}=$ phenotypic variance.

Confidence intervals (90 \%) were calculated after Knapp et al. (1985) as:

$\mathrm{UL}=1-\left[\mathrm{MS}_{3} / \mathrm{MS}_{2} * \mathrm{~F}_{\mathrm{UL}}(.10, \mathrm{v} 1\right.$ and v2 df) $]-1$

$\mathrm{LL}=1-\left[\mathrm{MS}_{3} / \mathrm{MS}_{2} * \mathrm{~F}_{\mathrm{LL}}(.90, \mathrm{v} 1\right.$ and $\left.\mathrm{v} 2 \mathrm{df})\right]-1$

Where: $\mathrm{UL}=$ upper limit of the confidence interval, $\mathrm{MS}_{3}=$ entry mean square, $\mathrm{MS}_{2}=$ residual mean square, $\mathrm{F}_{\mathrm{UL}}$ and $\mathrm{F}_{\mathrm{LL}}=\mathrm{F}$ value for the upper and lower limits calculated using the FINV function of Microsoft Excel (2007).

Proc CORR (SAS 2002) was used to analyze the relationship among traits on an entry mean basis. Entry means were plotted using Microsoft Excel (2007) to study the relationship among traits and calculate $\mathrm{R}^{2}$.

\section{Selection simulation}

Selection was simulated to estimate genetic gain for various selection criteria. LEX2010 was treated as the selection environment and LEX2011 was treated as the validation environment for FHB traits. For agronomic traits, an average of LEX2011 and PRN2011 was used as the validation environment. Quality traits were validated in PRN 2011. The mean of the selected lines after one cycle of selection in 2011 was compared to the mean of the population in the absence of selection in 2011.

\section{Results and discussion}

\section{Weather conditions and disease levels}

Wet and warm conditions were favorable for Fusarium graminearum infection in the three environments with precipitation levels much higher than historical averages during flowering (Figures A.3.1 to A.3.3). FHB traits varied among and within populations (Table 3.1). As expected, susceptible parents showed higher disease levels than the resistant parent VA01W-476 in both years. Minimum DON levels lower than the resistant parent, indicative of transgressive segregants, were observed in three 
populations in 2010 and in two populations in 2011. Disease levels in 2011 were higher than in 2010 both in the five populations and the parents used as checks (Table 3.1). Moderate to high levels of leaf rust (Puccinia triticina) and leaf blotch (Septoria tritici and Stagnospora nodorum) were observed in yield plots in LEX 2010. In 2011, leaf rust symptoms were negligible with low to moderate ratings of leaf blotch and FHB in both locations. Moderate to high and high levels of glume blotch (Stagnospora nodorum) were observed in LEX and PRN 2011, respectively (data not shown). Even though no significant differences for non-FHB diseases were found between homozygous susceptible and resistant lines at each QTL, it is reasonable to assume a certain level of yield reduction associated with foliar diseases and of test weight reduction associated with both foliar diseases and glume blotch.

\section{Fhb1 effects on FHB traits}

Fhb1 effects were studied on a class mean basis (susceptible, S; resistant, R) for each population averaged across 2010 and 2011 (Table 3.2). In all populations, there was a significant reduction in FHB traits when comparing $\mathrm{S}$ to $\mathrm{R}$ classes. The presence of Fhb1 resistant alleles significantly reduced FDK by $32 \%$ on average. These results are comparable to the 31, 32 and 27\% FDK reductions reported by Cardwell (2011), Agostinelli et al. (2012) and Pumphrey et al. (2007), respectively. This QTL reduced DON by an average of $20 \%$, similar to the $25 \%$ observed by Agostinelli et al. (2012) and lower than the $40 \%$ reported by Cardwell (2011). Index was significantly reduced by $28 \%$ on average in populations $2-5$. This generalized Fhbl-associated FDK and DON reduction levels indicates that exotic resistance is effective in lowering FHB impact in diverse genetic backgrounds. In this study, Fhb1 tended to be more effective in lowering DON in populations derived from single crosses (populations 4 and 5) than in the three way crosses (populations 1, 2 and 3). Fhbl-associated FDK and DON reduction levels were higher in 2010 (Table A.3.5) than in 2011 (Table A.3.6), when disease pressure was the highest of the two years. For this reason, it might be expected that under lower natural field infection levels, Fhbl would show better levels of FHB control than the results we observed in the inoculated scab nursery with extremely high disease pressure. 


\section{QFhs.nau-2DL effects on FHB traits}

A similar analysis was conducted to study the effects of $Q F h s . n a u-2 D L$ classes (Table 3.3). For this QTL, high frequencies of heterozygous $(\mathrm{H})$ lines were present in the five populations. Some $\mathrm{H}$ lines were as resistant as $\mathrm{R}$ lines while others were as susceptible as $\mathrm{S}$ lines; no clear pattern was evident. For example, DON level in $\mathrm{H}$ lines in populations 1 and 4 were statistically the same as in the R lines. On the other hand, DON levels in $\mathrm{H}$ lines in populations 2 and 5 were the same as in the $\mathrm{S}$ lines. When comparing the $\mathrm{S}$ against the R classes, $Q F h$ s.nau- $2 D L$ significantly reduced FDK by $29 \%$ on average in populations 1 and 4. Other studies showed both higher $Q F h$ s.nau-2DL-associated FDK reduction (Agostinelli et al., 2012) and non-significant effects (Cardwell, 2011). In the present study a significant $(\mathrm{p}<0.05) 24 \%$ DON average reduction was observed in all populations with the exception of population 3 . These DON reductions were lower than the $50 \%$ reported in other studies under both similar and lower levels of FHB pressure (Cardwell, 2011; Agostinelli et al., 2012). Although average QFhs.nau-2DL associated DON reduction in this study was similar to that associated with $F h b 1$, when looking at each population individually, $Q F h$ s.nau- $2 D L$ was more stable across populations and reduced DON by $21-27 \%$, whereas $F h b 1$ ranged from 12-27\% (Table 3.3). In 2011, under higher disease pressure, $Q F h$ s.nau-2DL was associated with a more generalized response in DON reduction (4 of 5 populations) than in 2010 ( 2 of 5 populations) (Tables A.3.8 and A.3.9). However, the largest DON reduction level (43\%) was observed in 2010 (Table A.3.8), whereas in 2011 DON reduction associated with this QTL ranged from 17$27 \%$ (Table A.3.9). In the present study, significant differences between homozygous $S$ and $\mathrm{R}$ lines at $Q F h$ s.nau-2DL were detected in a larger number of genetic backgrounds at higher FHB pressure conditions (Lexington 2012). No significant differences associated with this QTL were found for FHB index.

\section{Agronomic traits}

Higher mean yields were observed in PRN2011 than in LEX2010 and LEX2011 (Table 3.4). The susceptible parent, VA01W-476, was always out-yielded by the other parents. Heading date was the least variable of agronomic traits; population means ranged 
from 125.2 to 127.0 Julian days (A.3.14). Transgressive segregants were found for all agronomic traits in the five populations (Table 3.4)

\section{Fhb1 effects on agronomic traits}

Yield was significantly higher for the R-Fhbl class in populations 2, 3 and 5, by 5,8 and $2 \%$, respectively (Table 3.2 ). Most of the effects on TWT were in the desired direction but very small in absolute value. In contrast with previous reports of Fhb1 associated with increased plant height, in this study mean height was reduced for the $\mathrm{R}$ class in two populations and increased in only one population. These differences were less than $2 \%$ (Table A.3.18). Although heading date was significantly affected in three of four populations $(\mathrm{p}<0.05$ in populations 1 and 2 , and $\mathrm{p}<0.01$ in population 3$)$, differences were less than one Julian day (Table A.3.18).

\section{QFhs.nau-2DL effects on agronomic traits}

On average, $\mathrm{H}$ lines sometimes performed as $\mathrm{S}$ and sometimes as $\mathrm{R}$ lines for yield (Table 3.3). For example, in population 3 where yield was 5\% lower for the R class than for the $\mathrm{S}$ class, the $\mathrm{H}$ class was not significantly different from the $\mathrm{R}$ one. In population 4 , a similar scenario was observed, but with 3\% yield increment for both $\mathrm{R}$ and $\mathrm{H}$ classes. Population 1 presented a different situation where the $H$ class out-yielded the other classes by $2 \%$. Although statistically significant, these differences were very small in absolute value (100-200 kg/ha) with little agronomic significance. QFhs.nau-2DL increased plant height in two populations but it was associated with height reduction in

other populations (data not shown). Although heading date was significantly delayed in two populations $(\mathrm{p}<0.05)$, the impact was less than 1 Julian day. In population 2 , the $\mathrm{R}$ QFhs.nau-2DL class was 2 days earlier than the S class (data not shown).

\section{Fhb1 effects on milling and baking quality}

Mean population flour yield, softness equivalent and GPI are presented in table 4 with their corresponding ranges and parental means. In these genetic backgrounds, Fhbl impact on quality traits was very small and varied among populations (Table 3.2). Although significant reductions were found between $\mathrm{S}$ and $\mathrm{R}$ Fhb1-classes in all five 
populations for flour yield, differences were $1 \%$ or less. Resistant $F h b 1$ alleles were associated with higher softness equivalent in population $1(3 \%)$, and 1-2\% lower softness equivalent levels in the other four populations. More importantly, mean softness equivalent in each QTL class was within the acceptable 50-60\% minimum range for this trait (Everts et al., 2001) and the QTL effects were very small in absolute values. Gluten performance index was $4 \%$ higher for the R-Fhb1 class in population 2 , which reflects a better balance between lactic acid, sucrose and sodium carbonate SRC, but had the opposite impact by $2 \%$ in populations 1 and 3 . In a different genetic background, Cardwell (2011) found no significant differences between S and R lines for Fhbl for quality traits such as softness equivalent, flour yield, flour protein, and the different SRC tests. In hard spring wheat, FHB-resistant QTL effects on quality traits varied according to the QTL, the source of the QTL and the population to which the QTL was introgressed (McCartney et al., 2007). In that context, Fhb1-R alleles were associated with a slight reduction in flour yield and a reduction in falling number in one of the three genetic backgrounds evaluated. No significant impact was found on other traits such as TWT, weight of 1000 kernels, grain protein concentration, SDS sedimentation and mixograph parameters.

The present study shows a consistent association of Fhbl with effective FHB levels reduction and negligible impact on agronomic and quality traits that highlights its usefulness in breeding programs.

\section{QFhs.nau-2DL effects on milling and baking quality}

As observed in the Fhb1 analysis, in general, QFhs.nau-2DL had small effects on quality traits (Table 3.3). Although $Q F h$ s.nau-2DL effects on flour yield were significant in most populations, this effect was by $1 \%$ or less. The R-QFhs.nau- $2 D L$ class showed both reduction and increase in softness equivalent. Once again, all softness equivalent means were within the acceptable 50-60\% minimum range for this trait (Everts et al., 2001). Gluten performance index was only affected in population 4 and in a positive manner. Cardwell (2011) found that the presence of R-QFhs.nau-2DL alleles were associated with a $3 \%$ decline in lactic acid SRC that translated into a $1 \%$ reduction in GPI. This QTL was also associated with an 11\% lower milling quality score and softness 
equivalent, an $8 \%$ lower baking quality score and a $2 \%$ reduction in flour yield. With the exception of population 3, QFhs.nau-2DL was slightly more effective than Fhb1 in DON reduction (24 vs. $20 \%$ ) with negligible impact on agronomic and quality traits.

\section{Heritability estimates}

Broad sense heritabilities and their corresponding confidence intervals were estimated on an entry mean basis for each population separately as described earlier (Table 3.5). DON $\mathrm{h}^{2}$ ranged from 0.59 to 0.75 which are lower than the estimates reported by Agostinelli et al. (2012). Fusarium damaged kernels $\mathrm{h}^{2}$ estimates were moderate to high (0.58 to 0.82 ), lower than those reported by Agostinelli et al. (2012) and higher than reported by Verges et al. (2006). Fusarium head blight index $\mathrm{h}^{2}$ estimates were more variable among populations and less accurate which suggests that selection based on DON or FDK should be more effective than that based on FHB index.

Heritability of quality traits was high both overall and in each individual population in agreement with estimates reported in the literature (Smith et al., 2011). Flour yield and softness equivalent presented $\mathrm{h}^{2}$ greater than or equal to 0.85 . GPI was also highly heritable and ranged from 0.95 to 0.80 among the different populations.

\section{Combined effects of Fhb1 and QFhs.nau-2DL}

To investigate the effects of both QTL combined, all five populations were combined to ensure a large enough sample size for each Fhbl and QFhs.nau-2DL class combination. Six classes were compared in total where the first letter of the class combination corresponds to Fhbl ( $\mathrm{S}$ or R), and the second to QFhs.nau-2DL ( $\mathrm{S}, \mathrm{H}$ or R). The RR class, for example, comprised genotypes homozygous for resistant alleles at both loci. The SS (i.e., homozygous for susceptible alleles at both loci) combination was significantly higher in FDK and DON than all other possible combinations. The effect of resistance alleles at both QTL (RR class) resulted in a significant reduction by 50, 40 and $30 \%$ for FDK, DON, and FHB index when compared against the double susceptible class (SS). RR genotypes presented an additional 10\% reduction in DON when compared against $\mathrm{RH}$, and an additional $10 \%$ reduction in both FDK and DON, when compared against RS lines (data not shown). Significant QTL*year interaction was found in 
populations 3, 4 and 5 for $F h b 1$ and 1 and 5 for $Q F h s$.nau-2DL. However, when 2010 means were plotted against 2011 means, double resistant genotypes tended to cluster within the lower FDK and DON levels (Figure 3.1).

One of the most vexing questions facing breeders is whether QTL will impart sufficient resistance to progeny in the absence of native resistance. In this study, lines KY97C-0574-01 and 25R58 used in population 1, are good examples of highly susceptible parents with no apparent native resistance. In this population, Fhbl reduced FDK and DON by 23 and 12\%, respectively (Table 3.2), and $Q F h$ s.nau- $2 D L$ reduced both FHB traits by $24 \%$ (Table 3.3). Population 4 is another example that evidences that resistance derived from these exotic QTL could be sufficient. The parent 25R78 used in this population is also highly susceptible and in this context, Fhbl reduced FDK and DON by 34 and $26 \%$, respectively (Table 3.2), and QFhs.nau-2DL reduced both FHB traits by 35 and $22 \%$, respectively (Table 3.3). Trangressive segregates for DON or segregates that are close in DON and FDK to VA01W-476 support this idea of exotic resistance being enough to reduce FHB levels in well adapted material. For example, populations 2-5 and 1-5 showed lower FDK levels than VA01W-476 in 2010 and 2011, respectively (Table 1). Minimum DON levels found in populations 3, 4 and 5 in 2010 were essentially the same as those of VA01W-476 DON levels. In 2011, minimum DON levels in populations 4 and 5 were consistent with 2010, showing slightly lower levels than VA01W-476, and minimum DON level in population 1 was close to the resistant parent as well (Table 3.1).

\section{DON and FDK assessment}

Both DON and FDK are expensive and time consuming to quantify; thus, rapid and non-destructive methods for predicting these traits are of great interest. Near-infrared reflectance detects the absorption response by overtone and combination frequencies of O-H, C-H, and N-H molecular vibrations (Approved Method 39-00.01, 39-25.01, AACC, 2011) as well as physical properties like grain shape, size and color (Peiris et al, 2010). With the appropriate calibration, an NIR instrument can measure FDK and DON on a whole kernel basis at the same time and 100 samples can be run in 3 hours. The regression of DON on FDK varied among populations $\left(\mathrm{R}^{2}=0.24-0.62\right.$, Figures A.3.5 to 
A.3.9) as well as NIRFDK-DON (Figures A.5.1 to A.5.5). NIRFDK improved DON predictions in 2 of five populations, indicating that the NIR instrument may be somewhat more accurate in distinguishing scabby from healthy kernels. The fact that the ability of FDK or NIRFDK to predict DON varied among populations illustrates a relative weakness of their predictive value. However NIR predictive ability can be easily improved by updating the calibration equations with samples of diverse genetic backgrounds and DON wet chemistry. Moreover, a more useful way to assess their value is to evaluate response to indirect selection as discussed in the selection simulation section.

\section{Selection simulation}

When only FHB resistance was taken into account in the phenotypic selection simulation, either directly on DON, or indirectly on NIRFDK or FDK, the majority of the lines kept were homozygous resistant for $F h b l$ with a lower frequency of R lines for QFhs.nau-2DL (Table 3.6). Moreover, when direct selection on DON or indirect selection based on NIRFDK or FDK was implemented at p=0.20 (top 20\% selected), very few Fhb1-S lines were retained (only 3 and 5). Visual rating and FHB index based selections retained a larger number of Fhb1-S lines ( 7 and 11, respectively) than the other indirect selection criteria. The number of $Q F h s . n a u-2 D L-\mathrm{R}$ lines retained was in most cases lower than the number of QFhs.nau-2DL-H or S lines. This indicates that in these diverse genetic backgrounds, DON reduction was more closely associated with $F h b 1$ than with QFhs.nau-2DL. This contrasts with a previous study (Agostinelli et al., 2012) in which the effect of QFhs.nau-2DL was more pronounced than that of Fhbl and phenotypic selection enriched the population with a similar proportion of $\mathrm{R}$ lines for both QTL

Although direct phenotypic selection for lowering DON levels was the most effective strategy regardless of selection pressure, indirect selection using FDK and NIRFDK showed an intermediate genetic gain between direct selection on DON and indirect selection on visual rating and FHB index in the next generation. Among the phenotypic selection criteria, visual rating and FHB index showed the lowest DON reduction (Table 3.6) indicating that these traits are less effective for selecting for 
resistance to DON contamination. Although less effective, visual ratings and FHB index are less expensive than DON assessments; the data is available before harvest and there is no need to exhaustively clean samples to separate grain from chaff as is required for DON, FDK or NIRFDK determinations.

A genotypic selection scenario was conducted by retaining only the $\mathrm{R}$ lines at both Fhbl and QFhs.nau-2DL (i.e. RR genotypes). Under this scenario, DON was reduced less than under phenotypic selection using DON, FDK, NIRFDK or FHB index (Table 3.6). Genotypic selection was less effective than direct phenotypic selection to lower DON, in agreement with Agostinelli et al (2012) who found that retaining the 30\% lower DON lines equalized retaining all R lines for both Fhbl and QFhs.nau-2DL (12\% of the population).

A more realistic selection scenario was simulated by first retaining lines with desirable agronomic characteristics. As short early lines $(<93 \mathrm{~cm}$ tall, $\leq 130$ Julian days heading date) with $\geq 70.8 \mathrm{~kg} / \mathrm{hl}$ TWT were considered acceptable, the population size was reduced from 155 to 125 . From this subpopulation $(n=125)$, the top 25\% high yielding lines were selected $(n=31)$. Under this scenario, mean DON levels of the selected population remained the same as the non-selected population (21 ppm), suggesting that yield and susceptibility to FHB are not necessarily associated in this genetic background (Figure 3.2). In agreement with these results, correlation coefficients between yield and DON was weak $(r=0.20)$ in both years. Four of these 31 lines were in common with the top 20\% subpopulation selected for low DON (Table 3.6). These four lines identified with low DON constituted the $13 \%$ of the 31 lines that had been selected for high yield. By selecting these 4 lines, grain yield increased by $8 \%$ and DON was reduced by $30 \%$ (Figure 3.2). It is important to highlight that the four lines are high yielding and have low DON levels. Moreover, two lines carry homozygous R alleles at both Fhb1 and QFhs.nau-2DL (RR), and the other two lines carry homozygous R alleles at Fhbl (RS) or QFhs.nau-2DL (SR). Quality traits for these four lines were within the acceptable ranges for soft wheat for the most part. One of the four lines presented acceptable flour yield, softness equivalent and GPI. The other three lines presented acceptable quality with the exception of a $5 \%$ lower flour yield in one of the lines, a $4 \%$ lower softness equivalent in a different line and a 5\% lower GPI in the third one (data not 
shown). These results suggest that by building good quality from the selection of the parental material and including some sort of rapid assessment of quality in earlier generations to enrich the population with the desired quality parameters, plant breeders can succeed in selecting for resistance to FHB using the exotic QTL evaluated in this study while preserving the quality standards and improving yield.

\section{Conclusions}

Fhb1-derived resistance reduced FDK and DON, the two most direct measurements of FHB impact, in all five populations by 32 and $20 \%$, on average. Index was also reduced by $28 \%$ in four of five populations. $Q F h s . n a u-2 D L$ reduced FDK by $29 \%$ on average in two of five populations, and DON by $24 \%$ on average in four of five populations. Fhbl effects on yield were significant and positive, but small in absolute value ( $5 \%$ increase on average in 3 populations). Fhbl effects on TWT were also small and ranged from a $1 \%$ reduction to a $2 \%$ increase. In four of five populations $F h b 1$ was associated with increases in TWT. In four of five populations $Q F h s . n a u-2 D L$ effects on TWT were positive. In this study, the exotic resistance conferred by Fhb1 and QFhs.nau$2 D L$ was effective in reducing DON in all populations with the exception of population 3 where no significant differences were found between homozygous $\mathrm{S}$ and $\mathrm{R}$ QFhs.nau$2 D L$ classes. The effects of these QTL on agronomic and quality traits appear to be small and depend on the genetic background; negative effects can be balanced with adequate preservation of genetic variation and selection in the desired direction. High heritability of quality traits reported here and in other studies indicates that it is feasible to select for the desirable end use quality while selecting for FHB resistance. One cycle of either direct or indirect simulated phenotypic selection was effective in reducing DON levels and enriching the population with Fhb1 homozygous resistant lines. 
Table 3.1: Mean, Maximum (Max) and Minimum (Min) Fusarium damaged kernels (FDK), deoxynivalenol level (DON), and FHB index for five wheat populations and their parents in the Lexington, KY scab nursery in 2010 and 2011.

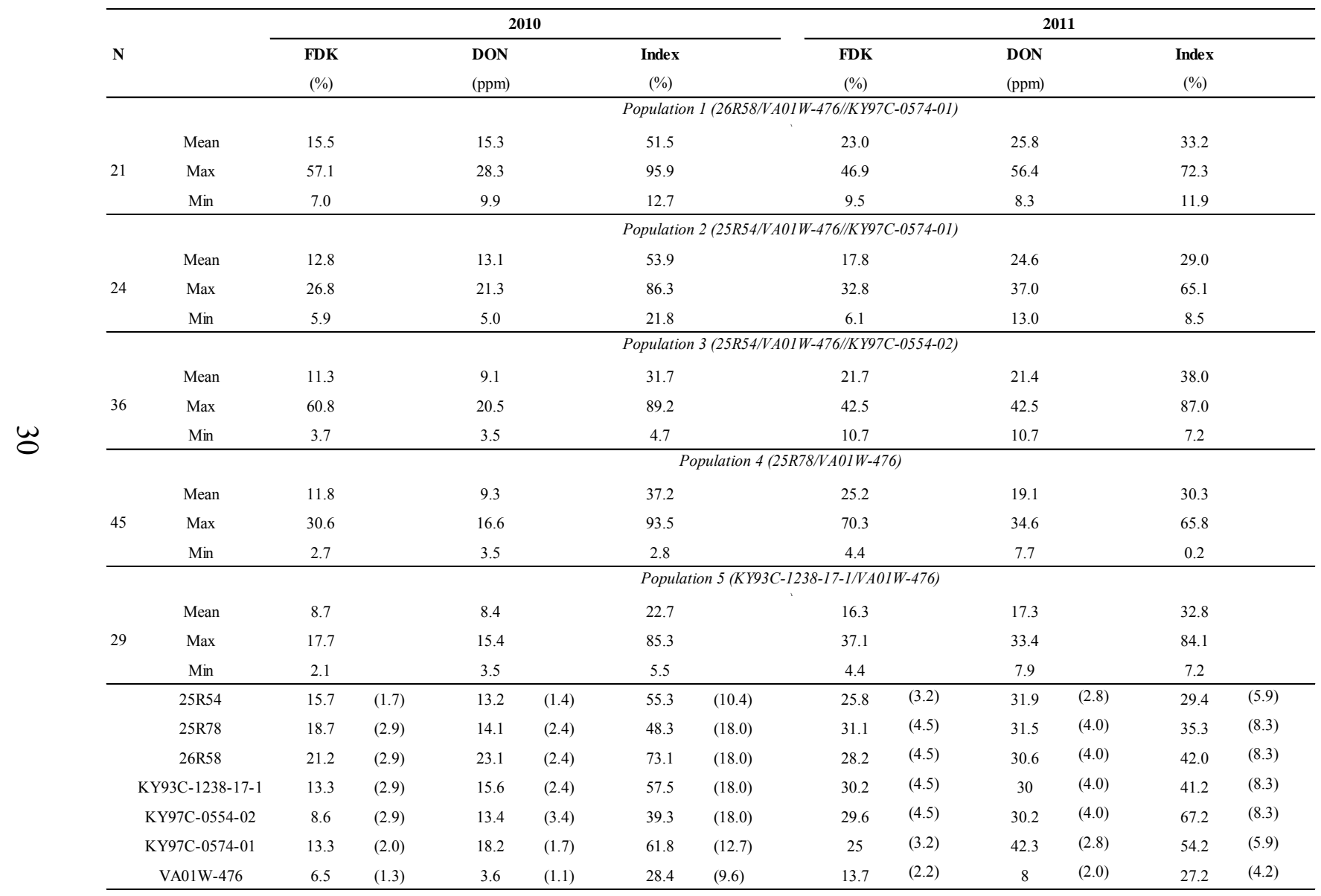

Numbers in parentheses are standard errors (SE) 
Table 3.2: Mean Fusarium damaged kernels (FDK), deoxynivalenol levels (DON), FHB index, yield, test weight (TWT), flour yield (FY), softness equivalent (SEQ) and gluten performance index (GPI) for wheat lines homozygous resistant (R) and susceptible (S) alleles at Fhb1, Lexington 2010 and 2011, and Princeton 2011.

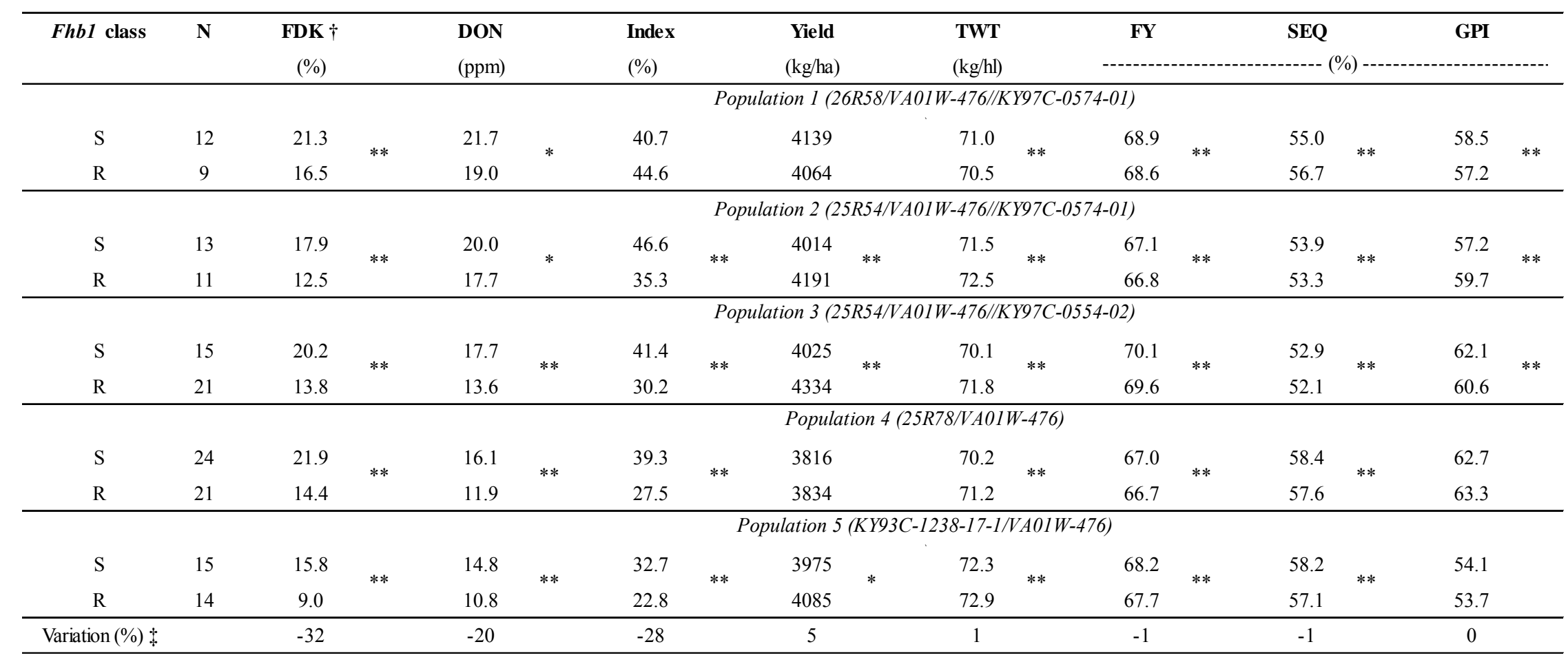

*,** (Difference between $\mathrm{S}$ and $\mathrm{R}$ classes significant at $\mathrm{P}<0.05,0.01$, respectively)

$\dagger$ FDK, DON, FHB index measured in 2010, 2011 Lexington scab nursery. Yield and TWT measured in Lexington 2010 and 2011, and Princeton 2011. FY, SEQ and GPI measured in Lexington 2010 and Princeton 2011.

$\$$ Mean variation based on significant differences between QTL classes. 
Table 3.3: Mean Fusarium damaged kernels (FDK), deoxynivalenol levels (DON), FHB index, yield, test weight (TWT), flour yield (FY), softness equivalent (SEQ) and gluten performance index (GPI) for homozygous susceptible (S), resistant (R) heterozygous (H) and wheat lines at QFhs.nau-2DL, Lexington 2010 and 2011, and Princeton 2011.

\begin{tabular}{|c|c|c|c|c|c|c|c|c|c|c|c|c|c|c|c|c|c|}
\hline \multirow{3}{*}{$\begin{array}{c}\begin{array}{c}\text { QFhs.nau-2DL } \\
\text { class }\end{array} \\
\mathrm{S}\end{array}$} & \multirow{3}{*}{$\begin{array}{l}\mathbf{N} \\
\\
9\end{array}$} & \multicolumn{2}{|l|}{$\begin{array}{c}\text { FDK } \dagger \\
(\%)\end{array}$} & \multicolumn{2}{|l|}{$\begin{array}{l}\text { DON } \\
(\mathrm{ppm})\end{array}$} & \multicolumn{2}{|l|}{$\begin{array}{l}\text { Index } \\
(\%)\end{array}$} & \multicolumn{2}{|c|}{$\begin{array}{r}\text { Yield } \\
(\mathrm{kg} / \mathrm{ha}) \\
\end{array}$} & \multicolumn{2}{|c|}{$\begin{array}{l}\text { TWT } \\
(\mathrm{kg} / \mathrm{hl}) \\
\end{array}$} & FY & 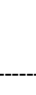 & \multicolumn{2}{|l|}{ SEQ } & \multicolumn{2}{|l|}{ GPI } \\
\hline & & \multicolumn{16}{|c|}{ Population 1 (26R58/VA01W-476//KY97C-0574-01) } \\
\hline & & 21.9 & $\mathrm{~b}$ & 21.9 & $\mathrm{~b}$ & 47.0 & $\mathrm{~b}$ & 4059 & a & 70.0 & a & 68.5 & a & 56.6 & $\mathrm{~b}$ & 57.1 & a \\
\hline $\mathrm{H}$ & 8 & 16.3 & a & 18.3 & a & 37.3 & a & 4193 & $\mathrm{~b}$ & 71.5 & $\mathrm{~b}$ & 69.0 & $\mathrm{~b}$ & 56.1 & a & 59.9 & $\mathrm{~b}$ \\
\hline $\mathrm{R}$ & 2 & 16.7 & a & 16.7 & a & 40.3 & $\mathrm{ab}$ & 3971 & $\mathrm{a}$ & 71.4 & $\mathrm{~b}$ & 68.3 & a & 55.7 & a & 57.8 & a \\
\hline & & \multicolumn{16}{|c|}{ Population $2(25 R 54 / V A 01 W-476 / / K Y 97 C-0574-01)$} \\
\hline $\mathrm{S}$ & 4 & 14.5 & a & 20.6 & $\mathrm{~b}$ & 37.1 & a & 4060 & a & 70.8 & a & 67.3 & $\mathrm{c}$ & 56.5 & $\mathrm{c}$ & 56.5 & a \\
\hline $\mathrm{H}$ & 15 & 15.6 & a & 19.3 & $\mathrm{~b}$ & 41.7 & $\mathrm{a}$ & 4071 & $\mathrm{a}$ & 72.0 & $\mathrm{~b}$ & 66.9 & $\mathrm{~b}$ & 53.9 & $\mathrm{~b}$ & 59.6 & $\mathrm{~b}$ \\
\hline $\mathrm{R}$ & 4 & 14.1 & a & 15.0 & $\mathrm{a}$ & 42.1 & $\mathrm{a}$ & 4154 & $\mathrm{a}$ & 72.5 & $\mathrm{~b}$ & 66.5 & a & 50.4 & a & 56.7 & a \\
\hline & & \multicolumn{16}{|c|}{ Population $3(25 R 54 / V A 01 W-476 / / K Y 97 C-0554-02)$} \\
\hline $\mathrm{S}$ & 17 & 15.0 & a & 14.7 & a & 33.2 & $\mathrm{ab}$ & 4314 & $\mathrm{~b}$ & 71.7 & $\mathrm{~b}$ & 69.7 & $\mathrm{a}$ & 51.8 & a & 60.5 & a \\
\hline $\mathrm{H}$ & 9 & 18.6 & $\mathrm{~b}$ & 15.8 & $\mathrm{a}$ & 38.1 & $\mathrm{~b}$ & 4085 & $\mathrm{a}$ & 70.3 & a & 70.2 & $\mathrm{c}$ & 52.2 & $\mathrm{~b}$ & 63.6 & $\mathrm{~b}$ \\
\hline $\mathrm{R}$ & 7 & 16.2 & $\mathrm{ab}$ & 15.4 & $\mathrm{a}$ & 29.3 & $\mathrm{a}$ & 4071 & a & 70.7 & a & 70.0 & $\mathrm{~b}$ & 53.7 & $\mathrm{c}$ & 60.0 & a \\
\hline
\end{tabular}

Means (by population) within the same column followed by different letters are significantly different at $\mathrm{P}<0.05$

$\uparrow$ FDK, DON, FHB index measured in 2010, 2011 Lexington scab nursery. Yield and TWT measured in Lexington 2010 and 2011 , and Princeton 2011. FY, SEQ and GPI measured in Lexington 2010 and Princeton 2011. 
Table 3.3 (continued): Mean Fusarium damaged kernels (FDK), deoxynivalenol levels (DON), FHB index, yield, test weight (TWT), flour yield (FY), softness equivalent (SEQ) and gluten performance index (GPI) for homozygous susceptible (S), resistant (R) heterozygous (H) and wheat lines at QFhs.nau-2DL, Lexington 2010 and 2011, and Princeton 2011.

\begin{tabular}{|c|c|c|c|c|c|c|c|c|c|c|c|c|c|c|c|c|c|}
\hline \multirow[t]{2}{*}{$\begin{array}{l}\text { QFhs.nau-2DL } \\
\text { class }\end{array}$} & \multirow[t]{2}{*}{$\mathbf{N}$} & \multicolumn{2}{|l|}{$\begin{array}{c}\text { FDK † } \\
(\%)\end{array}$} & \multicolumn{2}{|l|}{$\begin{array}{l}\text { DON } \\
(\mathrm{ppm})\end{array}$} & \multicolumn{2}{|l|}{$\begin{array}{l}\text { Index } \\
(\%) \\
\end{array}$} & $\begin{array}{r}\text { Yield } \\
(\mathrm{kg} / \mathrm{ha})\end{array}$ & & \multicolumn{2}{|c|}{$\begin{array}{l}\text { TWT } \\
(\mathrm{kg} / \mathrm{hl})\end{array}$} & $\mathbf{F Y}$ & & \multicolumn{2}{|c|}{$\begin{array}{c}\text { SEQ } \\
-(\%)\end{array}$} & \multicolumn{2}{|l|}{ GPI } \\
\hline & & \multicolumn{16}{|c|}{ Population $4(25 R 78 / V A 01 W-476)$} \\
\hline $\mathrm{S}$ & 12 & 21.9 & $\mathrm{~b}$ & 16.3 & $\mathrm{~b}$ & 35.3 & a & 3756 & a & 70.1 & a & 66.9 & $\mathrm{~b}$ & 58.1 & $\mathrm{~b}$ & 62.4 & a \\
\hline $\mathrm{H}$ & 20 & 16.6 & a & 13.2 & a & 31.5 & a & 3868 & $\mathrm{~b}$ & 71.2 & $\mathrm{~b}$ & 66.8 & b & 57.7 & a & 62.7 & $a b$ \\
\hline $\mathrm{R}$ & 7 & 14.3 & a & 12.7 & a & 34.5 & a & 3903 & $\mathrm{~b}$ & 71.0 & $\mathrm{~b}$ & 66.6 & a & 57.7 & $\mathrm{ab}$ & 63.6 & $\mathrm{~b}$ \\
\hline & & \multicolumn{16}{|c|}{ Population 5 (KY93C-1238-17-1/VA01W-476) } \\
\hline $\mathrm{S}$ & 6 & 11.4 & a & 13.3 & $\mathrm{~b}$ & 23.5 & a & 4025 & a & 73.0 & $\mathrm{~b}$ & 67.8 & $\mathrm{~b}$ & 57.3 & a & 54.4 & a \\
\hline $\mathrm{H}$ & 15 & 14.0 & $\mathrm{~b}$ & 14.0 & $\mathrm{~b}$ & 31.0 & $\mathrm{~b}$ & 4053 & a & 72.6 & $\mathrm{~b}$ & 68.3 & c & 57.6 & a & 54.0 & a \\
\hline $\mathrm{R}$ & 8 & 10.5 & a & 10.5 & a & 25.1 & $\mathrm{ab}$ & 3984 & a & 72.1 & $\mathrm{a}$ & 67.4 & a & 58.1 & $\mathrm{~b}$ & 53.5 & a \\
\hline Variation $(\%) \ddagger$ & & -29 & & -24 & & 0 & & -1 & & 1 & & 0 & & -2 & & 2 & \\
\hline
\end{tabular}

Means (by population) within the same column followed by different letters are significantly different at $\mathrm{P}<0.05$

$\uparrow$ FDK, DON, FHB index measured in 2010, 2011 Lexington scab nursery. Yield and TWT measured in Lexington 2010 and 2011 , and Princeton 2011. FY, SEQ and GPI measured in Lexington 2010 and Princeton 2011.

† Mean variation based on significant differences between QTL classes. 
Table 3.4: Mean, Maximum (Max) and Minimum (Min) yield and test weight (TWT) for five wheat populations and their parents in Lexington (LEX) 2010 and 2011, and Princeton (PRN) 2011. Mean flour yield (FY), softness equivalent (SEQ) and gluten performance index (GPI) for LEX 2010 and PRN 2011.

\begin{tabular}{|c|c|c|c|c|c|c|c|c|c|c|}
\hline \multirow{3}{*}{$\mathbf{N}$} & & \multicolumn{2}{|c|}{ LEX 2010} & \multicolumn{2}{|c|}{ LEX 2011} & \multicolumn{2}{|c|}{ PRN 2011} & \multicolumn{3}{|c|}{ LEX 2010 and PRN 2011} \\
\hline & & Yield & TWT & Yield & TWT & Yield & TWT & $\mathbf{F Y}$ & SEQ & GPI \\
\hline & & (kgha) & $(\mathrm{kghl})$ & (kg/ha) & $(\mathrm{kghl})$ & (kgha) & $(\mathrm{kg} / \mathrm{hl})$ & & 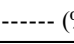 & -..-.. \\
\hline & & \multicolumn{9}{|c|}{ Population I (26R58/VA01W-476/KY97C-0574-01) } \\
\hline \multirow{4}{*}{21} & Mean & 4041.0 & 72.4 & 3899.0 & 68.9 & 4380.0 & 71.1 & 68.8 & 55.8 & 57.9 \\
\hline & Max & 4743.0 & 75.8 & 5114.0 & 74.1 & 5249.0 & 74.3 & 71.8 & 61.9 & 65.6 \\
\hline & Min & 3084.0 & 67.9 & 2114.0 & 63.0 & 2263.0 & 66.6 & 66.3 & 45.5 & 46.1 \\
\hline & & \multicolumn{9}{|c|}{ Population $2(25 R 54 / V A 01 W-476 / / K Y 97 C-0574-01)$} \\
\hline \multirow{4}{*}{24} & Mean & 4117.0 & 72.2 & 3839.0 & 71.4 & 4339.0 & 72.2 & 66.9 & 53.6 & 58.4 \\
\hline & $\operatorname{Max}$ & 4886.0 & 76.1 & 5085.0 & 74.8 & 5419.0 & 76.0 & 69.2 & 62.0 & 66.5 \\
\hline & Min & 2237.0 & 66.4 & 2440.0 & 62.1 & 2966.0 & 63.3 & 63.6 & 46.5 & 49.4 \\
\hline & & \multicolumn{9}{|c|}{ Population $3(25 R 54 /$ VA01W-476//KY97C-0554-02) } \\
\hline \multirow{4}{*}{36} & Mean & 4069.0 & 71.8 & 4059.0 & 69.7 & 4486.0 & 71.7 & 69.8 & 52.4 & 61.2 \\
\hline & Max & 5307.0 & 75.8 & 5367.0 & 74.6 & 5471.0 & 75.0 & 73.2 & 63.9 & 74.6 \\
\hline & Min & 2634.0 & 65.3 & 1758.0 & 58.8 & 3044.0 & 67.0 & 65.9 & 42.2 & 49.7 \\
\hline & & \multicolumn{9}{|c|}{ Population 4 (25R78/VA01W-476) } \\
\hline \multirow{4}{*}{45} & Mean & 4055.0 & 72.4 & 3492.0 & 68.7 & 3927.0 & 70.9 & 66.9 & 58.1 & 62.9 \\
\hline & Max & 5219.0 & 76.8 & 4971.0 & 74.6 & 5003.0 & 75.5 & 69.0 & 64.3 & 75.9 \\
\hline & Min & 2661.0 & 67.6 & 1846.0 & 57.1 & 1598.0 & 60.8 & 63.5 & 50.7 & 50.5 \\
\hline & & \multicolumn{9}{|c|}{ Population $5($ KY93C-1238-17-1/VA01W-476) } \\
\hline \multirow{3}{*}{29} & Mean & 3626.0 & 83.6 & 4075.0 & 70.3 & 4383.0 & 72.8 & 67.9 & 57.7 & 53.8 \\
\hline & Max & 4536.0 & 99.1 & 5328.0 & 74.9 & 5157.0 & 76.4 & 71.7 & 63.6 & 64.2 \\
\hline & Min & 2584.0 & 68.6 & 3031.0 & 65.3 & 3326.0 & 65.6 & 65.2 & 51.0 & 46.5 \\
\hline
\end{tabular}


Table 3.4 (continued): Mean, Maximum (Max) and Minimum (Min) yield and test weight (TWT) for five wheat populations and their parents in Lexington (LEX) 2010 and 2011, and Princeton (PRN) 2011. Mean flour yield (FY), softness equivalent (SEQ) and gluten performance index (GPI) for LEX 2010 and PRN 2011.

\begin{tabular}{|c|c|c|c|c|c|c|c|c|c|c|c|c|c|c|c|c|c|c|}
\hline & \multicolumn{4}{|c|}{ LEX 2010} & \multicolumn{4}{|c|}{ LEX 2011} & \multicolumn{4}{|c|}{ PRN 2011} & \multicolumn{6}{|c|}{ LEX 2010 and PRN 2011} \\
\hline & $\begin{array}{c}\text { Yield } \\
(\mathrm{kg} / \mathrm{ha})\end{array}$ & & $\begin{array}{l}\text { TWT } \\
(\mathrm{kg} / \mathrm{hl})\end{array}$ & & $\begin{array}{c}\text { Yield } \\
(\mathrm{kg} / \mathrm{ha})\end{array}$ & & $\begin{array}{c}\text { TWT } \\
(\mathrm{kg} / \mathrm{hl})\end{array}$ & & $\begin{array}{c}\text { Yield } \\
(\mathrm{kg} / \mathrm{ha})\end{array}$ & & $\begin{array}{c}\text { TWT } \\
(\mathrm{kg} / \mathrm{hl})\end{array}$ & & FY & & $\begin{array}{l}\text { SEQ } \\
--(\%\end{array}$ & & GPI & \\
\hline $25 \mathrm{R} 54$ & 3774.5 & (140.7) & 69.0 & $(0.6)$ & 4054.5 & $(162.2)$ & 67.2 & $(0.6)$ & 4392.5 & (189.5) & 68.8 & $(0.5)$ & 70.1 & $(0.2)$ & 62.1 & $(0.5)$ & 60.7 & $(0.0)$ \\
\hline $25 \mathrm{R} 78$ & 5097.8 & $(214.8)$ & 71.8 & $(0.8)$ & 3372.3 & (229.3) & 66.6 & $(0.8)$ & 4578.6 & $(268.0)$ & 70.8 & $(0.6)$ & 69.2 & $(0.3)$ & 59.1 & $(0.7)$ & 58.6 & $(0.0)$ \\
\hline $26 \mathrm{R} 58$ & 4192.7 & $(214.8)$ & 66.7 & $(0.8)$ & 4283.7 & $(229.3)$ & 63.9 & $(0.8)$ & 5020.3 & $(268.0)$ & 68.2 & $(0.6)$ & 68.4 & $(0.3)$ & 58.2 & $(0.7)$ & 51.0 & $(0.0)$ \\
\hline KY93C-1238-17-1 & 4090.5 & $(214.8)$ & 72.7 & $(0.8)$ & 4504.3 & (229.3) & 67.0 & $(0.8)$ & 4990.6 & $(268.0)$ & 70.4 & $(0.6)$ & 70.5 & $(0.3)$ & 61.9 & $(0.7)$ & 47.3 & $(0.0)$ \\
\hline KY97C-0554-02 & 4998.3 & $(214.8)$ & 72.7 & $(0.8)$ & 4789.1 & (229.3) & 70.1 & $(0.8)$ & 4856.1 & $(268.0)$ & 70.2 & $(0.6)$ & 72.8 & $(0.3)$ & 48.0 & $(0.7)$ & 59.7 & $(0.0)$ \\
\hline KY97C-0574-01 & 4231.1 & (151.9) & 71.7 & $(0.6)$ & 4317.1 & $(162.2)$ & 69.6 & $(0.6)$ & 4800.5 & (189.5) & 72.1 & $(0.5)$ & 69.0 & $(0.2)$ & 56.5 & $(0.5)$ & 60.2 & $(0.0)$ \\
\hline VA01W-476 & 2946.1 & (107.4) & 74.5 & $(0.4)$ & 2881.0 & (114.7) & 71.6 & $(0.4)$ & 3482.0 & $(134.0)$ & 73.1 & $(0.3)$ & 64.8 & $(0.2)$ & 54.8 & $(0.4)$ & 65.3 & $(0.0)$ \\
\hline
\end{tabular}

Numbers in parentheses are standard errors (SE) 
Table 3.5: Heritabilities and their 90\% confidence intervals in parentheses, based on 2 year ANOVA of five wheat populations. Traits evaluated were: Fusarium damaged kernels (FDK), deoxynivalenol level (DON), FHB index, flour yield (FY), softness equivalent (SEQ) and gluten performance index (GPI), Lexington and Princeton, KY 2010-2011.

\begin{tabular}{|c|c|c|c|c|c|c|}
\hline & DON & FDK & Index & FY & SEQ & GPI \\
\hline Overall & $0.75(0.80-0.69)$ & $0.65(0.72-0.57)$ & $0.33(0.46-0.18)$ & $0.94(0.95-0.92)$ & $0.95(0.96-0.94)$ & $0.93(0.94-0.91)$ \\
\hline Population 1 & $0.71(0.84-0.48)$ & $0.82(0.90-0.68)$ & $0.51(0.73-0.12)$ & $0.85(0.92-0.74)$ & $0.93(0.96-0.88)$ & $0.95(0.97-0.91)$ \\
\hline Population 2 & $0.63(0.79-0.37)$ & $0.60(0.77-0.31)$ & $0.26(0.57--0.28)$ & $0.90(0.94-0.84)$ & $0.98(0.99-0.96)$ & $0.95(0.97-0.92)$ \\
\hline Population 3 & $0.59(0.74-0.37)$ & $0.63(0.76-0.43)$ & $0.41(0.62-0.08)$ & $0.95(0.97-0.93)$ & $0.98(0.99-0.96)$ & $0.93(0.95-0.89)$ \\
\hline Population 4 & $0.75(0.83-0.62)$ & $0.58(0.72-0.38)$ & $0.59(0.72-0.39)$ & $0.88(0.92-0.82)$ & $0.91(0.94-0.86)$ & $0.79(0.86-0.69)$ \\
\hline Population 5 & $0.59(0.75-0.34)$ & $0.67(0.80-0.47)$ & $0.66(0.79-0.44)$ & $0.93(0.96-0.88)$ & $0.89(0.93-0.82)$ & $0.80(0.88-0.67)$ \\
\hline
\end{tabular}


Table 3.6: Mean deoxynivalenol (DON) reduction after one cycle of simulated selection, proportion of the population selected (p), number of lines selected (n) for different selection criteria, and lines selected from the following genotypic categories: resistant for both Fhbl and QFhs.nau-2DL (RR), resistant for Fhbl and heterozygous for QFhs.nau-2DL (RH), resistant for Fhb1 and susceptible for QFhs.nau-2DL (RS), susceptible for Fhbl and R for QFhs.nau-2DL (SR), susceptible for both Fhbl and QFhs.nau$2 D L$ (SS), resistant for Fhbl and unknown for QFhs.nau-2DL (R?), and S for Fhbl and unknown for QFhs.nau-2DL (S?).

\begin{tabular}{|c|c|c|c|c|c|c|c|c|c|c|c|}
\hline \multirow[b]{2}{*}{ Selection criterion } & \multirow[b]{2}{*}{$\mathbf{p}$} & \multirow[b]{2}{*}{$\mathbf{n}$} & \multirow[b]{2}{*}{$\begin{array}{c}\text { DON reduction } \\
(\%)\end{array}$} & \multicolumn{8}{|c|}{ Lines selected from each category $\dagger$} \\
\hline & & & & $\begin{array}{l}\mathbf{R R} \\
(15)\end{array}$ & $\begin{array}{l}\text { RH } \\
\text { (29) }\end{array}$ & $\begin{array}{l}\text { RS } \\
(29)\end{array}$ & $\begin{array}{l}\text { SR } \\
\text { (13) }\end{array}$ & $\begin{array}{l}\text { SH } \\
(38)\end{array}$ & $\begin{array}{l}\text { SS } \\
(19)\end{array}$ & $\begin{array}{l}\mathbf{R} ? \\
\text { (3) }\end{array}$ & $\begin{array}{l}\mathbf{S} \underline{?} \\
(9)\end{array}$ \\
\hline Direct phenotypic selection & & & 28 & 8 & 10 & 9 & 1 & 2 & - & 1 & - \\
\hline \multicolumn{12}{|l|}{ Indirect phenotypic selection for: } \\
\hline FDK & ח 20 & 31 & 25 & 7 & 10 & 10 & 2 & 1 & - & - & 1 \\
\hline NIRFDK & & & 22 & 6 & 11 & 9 & 1 & 3 & 1 & - & - \\
\hline Visual rating & & & 18 & 5 & 10 & 8 & 1 & 5 & 1 & 1 & - \\
\hline FHB index & & & 18 & 7 & 4 & 9 & 3 & 6 & 2 & - & - \\
\hline Direct phenotypic selection & & & 36 & 5 & 4 & 4 & 1 & - & - & 1 & - \\
\hline \multicolumn{12}{|l|}{ Indirect phenotypic selection for: } \\
\hline FDK & & & 34 & 3 & 5 & 7 & - & - & - & - & - \\
\hline NIRFDK & 0.10 & 15 & 29 & 3 & 6 & 5 & - & - & 1 & - & - \\
\hline Visual rating & & & 11 & 2 & 6 & 3 & - & 2 & 1 & 1 & - \\
\hline FHB index & & & 26 & 4 & 3 & 7 & - & 1 & - & - & - \\
\hline Genotypic selection using Fhb1 and $Q F h s . n a u-2 D L$ & & & 19 & 15 & - & - & - & - & - & - & - \\
\hline
\end{tabular}

$\dagger$ Numbers in parentheses indicate the total number of lines that belong to each category combination of Fhbl and QFhs.nau-2DL before selection 
(a)

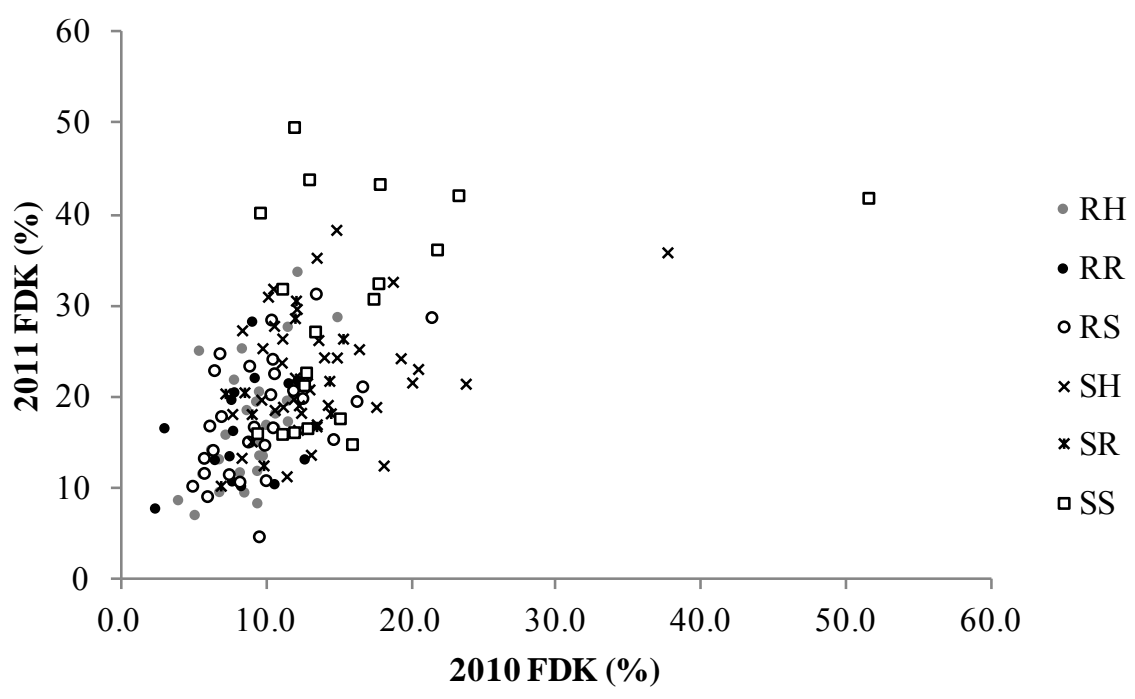

(b)

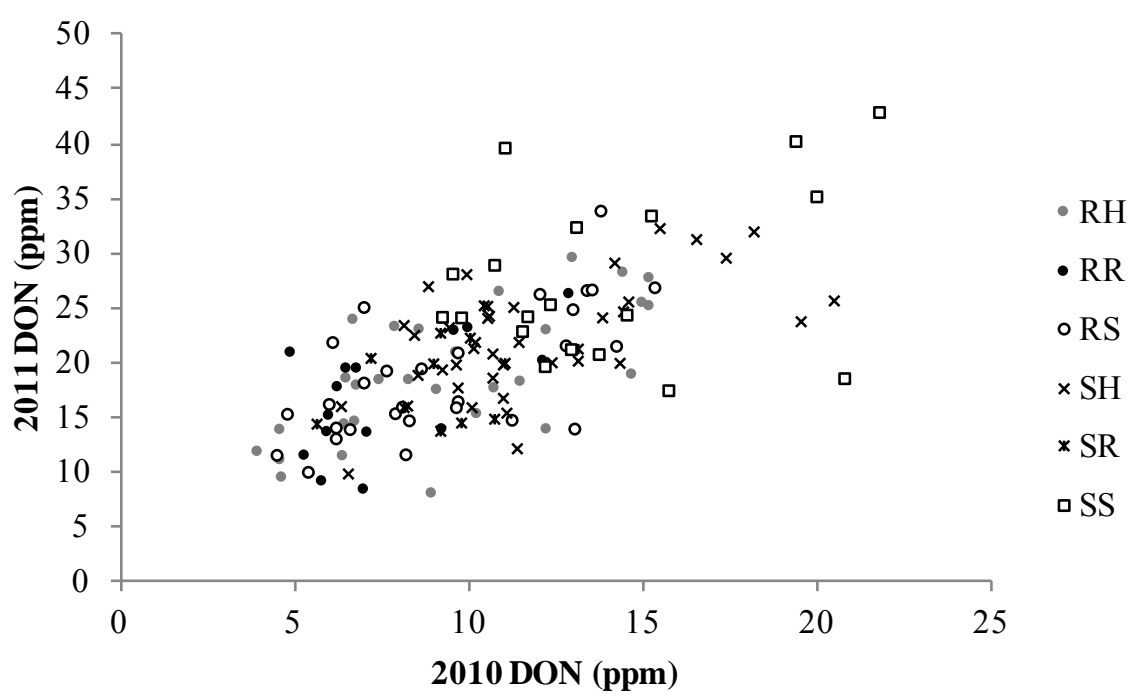

Figure 3.1: Relationship between 2010 and 2011 measurements of FDK (a) and DON (b) by QTL class combination. 


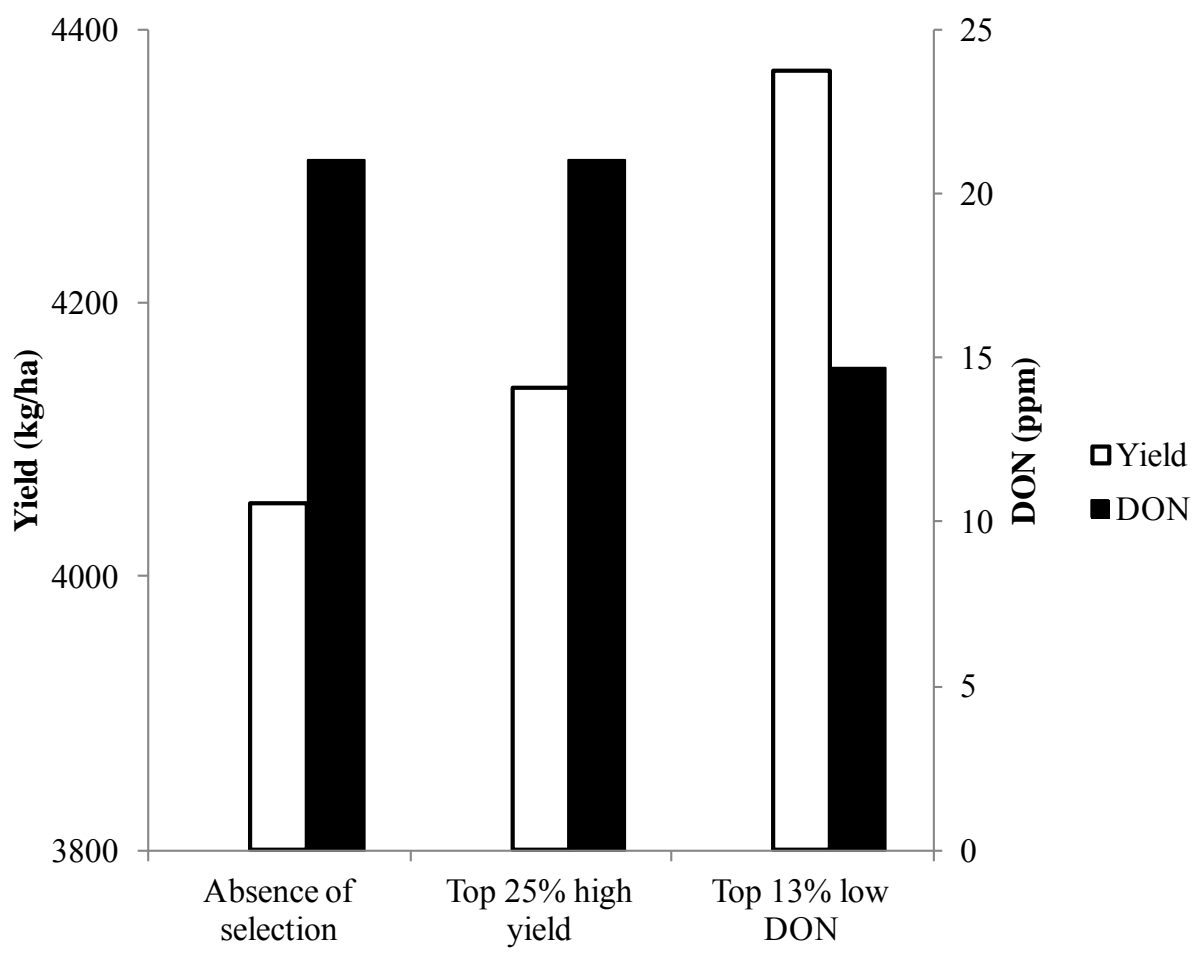

Figure 3.2: Mean yield (white bars) and DON levels (black bars) for wheat lines before selection for these traits (absence of selection), after the top high yielding $25 \%$ lines were selected and after a subsequent top $13 \%$ of the lines was selected for low DON. 


\section{Chapter Four}

Milling and baking quality predictions using wheat meal assays and near infrared reflectance

\section{Introduction}

Soft red winter (SRW) wheat is mainly used for cakes, cookies, crackers, donuts and flat breads (Beuerlein, 2001). For each specific end-use product there are specific composition and rheological functionality requirements. Milling quality is the result of flour yield and flour particle size. Baking quality is mainly a function of gluten strength and water absorption (Smith et al., 2011). Gluten strength, a function of protein concentration and protein composition (glutenin:gliadin) (Guttieri et al., 2001; Smith et al., 2011), is required in higher levels for crackers than for cookies (Gutierri et al., 2001).

The flour based solvent retention capacity (SRC) tests predict baking performance by measuring the weight of solvent retained as a percentage of the flour weight (Smith et al., 2011). Cross-linked polymeric materials in the flour swell in the presence of these four solvents and the degree of swelling are measured by changes in weight (Kweon et al., 2011). Sodium carbonate (5\% w/w), sucrose (50\% w/w), lactic acid $(5 \%)$ and water are used to predict the functional contribution of starch, pentosans, glutenins, and a combination of these three components, respectively (Kweon et al., 2011). As starch, pentosans and glutenins contribute to $\mathrm{P}_{\max }$ (peak of pressure) in a typical alveograph profile (Kweon et al., 2011), the different SRC tests provide more complete information about flour quality than the alveograph alone. Flour water SRC measures global water affinity of starch, arabinoxylans, gluten, and gliadins. This parameter is negatively correlated to flour yield and softness equivalent (Souza et al., 2011). Cookies and crackers made with flours with very high water retention require longer cooking time. This results in less tender products and higher costs of production (Guttieri et al., 2001). Flour sucrose SRC is a measure of arabinoxylans content that affects water absorption in baked products and it is considered the best predictor of cookie quality. There is a negative correlation between flour sucrose SRC and with wire-cut cookie diameter ( $\mathrm{r}=$ - 
$0.66)$, flour yield $(\mathrm{r}=-0.31)$ and softness equivalent $(\mathrm{r}=-0.23)$. Flour sodium carbonate SRC measures starch damage. The solvent used in this test ionizes the ends of starch polymers increasing its water binding capacity. This test predicts flour yield $(\mathrm{r}=-0.48)$ and cookie diameter $(\mathrm{r}=-0.22)$. The flour lactic acid SRC predicts gluten strength and it is correlated with the SDS sedimentation (SDS) test and flour protein concentration (Souza et al., 2011). The SDS test is used to select bread quality in hard wheat (Knott et al., 2009). The desired directions for flour water SRC, flour sodium carbonate SRC and flour sucrose SRC is towards lower values, whereas for lactic acid SRC it is towards larger values. Gold standard targets published by Kweon et al. (2011) for cookies and crackers are $\leq 51$ water SRC, $\leq 64$ sodium carbonate SRC, $\leq 89$ sucrose SRC and $\geq 87$ lactic acid SRC. For sponge-and-dough products the targets are $\leq 57$ water SRC, $\leq 72$ sodium carbonate SRC, $\leq 96$ sucrose SRC and $\geq 100$ lactic acid SRC. Souza et al. (2012) determined that selection to maintaining a quality type could be more efficient by focusing on a limited number of traits with large heritability values such as flour yield, softness equivalent, and SRC.

Knott et al. (2009) evaluated wheat meal (WM) assays for their ability to select lines with acceptable soft winter wheat quality in early generations. The advantages of WM assays are the small amount of grain required and that sample mills are relatively inexpensive. A previous study (Gutierri et al., 2004) showed a very good prediction ability of flour sodium carbonate SRC and flour sucrose SRC by WM-sodium carbonate $\mathrm{SRC}(\mathrm{r}=0.69-0.81$ and $\mathrm{r}=0.74-0.84$, respectively). The ability of predicting both flour SRC with only WM-sodium carbonate SRC test is beneficial both in terms of time and resources because sodium carbonate solvent is much less concentrated than the sucrose solvent. Wheat meal-SDS in their study show an excellent ability to predict flour lactic acid SRC ( $\mathrm{r}=0.74-0.93)$. Wheat-meal SDS test presents advantages as compared to flour lactic acid SRC because it requires only 1 gram of wheat meal and the laboratory procedure is less time consuming. Knott et al. (2009) estimated broad-sense heritability for WM sodium carbonate SRC and WM-SDS to be 0.70 and 0.67 , respectively. Wheat meal SDS was correlated to flour protein $(\mathrm{r}=0.29)$, lactic acid SRC $(\mathrm{r}=0.37)$ and wire-cut cookie measures $(\mathrm{r}=-0.42)$. Whole grain-wheat meal sodium carbonate $\mathrm{SRC}$ was correlated to flour yield $(\mathrm{r}=-0.40)$, flour sucrose $\mathrm{SRC}(\mathrm{r}=-0.37)$, flour sodium carbonate 
$\mathrm{SRC}(\mathrm{r}=0.46)$, flour water SRC $(\mathrm{r}=0.47)$ and wire-cut cookie diameter $(\mathrm{r}=-0.33)$. The researchers concluded that WM assays could be an easy and economical alternative to increase the proportion of experimental lines with desirable milling and baking quality in breeding programs.

Near infrared spectroscopy is of great interest for measuring quality parameters for its simplicity, time use efficiency and objectivity. If performed on whole grain samples, this method is of even more interest for breeders because it is non-destructive. As described in the AACC Approved Methods guidelines, the absorption response by overtone and combination frequencies of $\mathrm{O}-\mathrm{H}, \mathrm{C}-\mathrm{H}$, and $\mathrm{N}-\mathrm{H}$ molecular vibrations is discernible by photometric detectors, which inherently have a very high signal-to-noise response. Thus, the composition (protein, carbohydrate, moisture, lipid and others) of cereals can be determined (Approved Method 39-00.01, 39-25.01, AACC, 2011). For example, protein content in whole grain wheat samples is based on transmittance or reflectance of near-infrared (850-2,500 nm) energy (Approved Method 39-25.01, AACC, 2011). Physical properties, such us grain shape, size and color also impact the NIR absorption levels (Peiris et al., 2010). The USDA/ARS Soft Wheat Quality Laboratory (SWQL), at Wooster, OH, currently uses whole grain NIR to measure moisture, hardness and whole grain protein (http://www.ars.usda.gov/Research/docs.htm?docid=21522) and flour NIR to measure flour protein. Near infrared spectroscopy based predictions of these parameters, other milling quality traits such as softness equivalent, flour yield, and baking quality predictors such as SRC are of great value in breeding programs where quality phenotyping at the earlier stages of the cycle could help discard genotypes with undesirable quality with rapid inexpensive methods.

The objectives of this study were (i) to evaluate WM-SDS and WM-sodium carbonate SRC, and (ii) NIR for their ability to predict milling and baking quality.

\section{Materials and methods}

\section{Plant material}

Five sets of inbred lines derived from 2 and 3-way crosses were evaluated in this study (Table 4.1). Crosses were made between FHB susceptible parents (26R58, KY97C- 
0574-01, 25R54, KY97C, KY97C-0554-02, 25R78 and KY93C-1238-17-1) and FHBresistant VA01W-476, a doubled haploid line derived from the cross 'Roane'/W14. These crosses represent typical resistant $\mathrm{x}$ susceptible crosses used in SRW wheat breeding programs. The populations were grown in six row plots $3 \mathrm{~m}$ long, replicated three times in a RCB design in two environments: Lexington 2010 (LEX10) and Princeton 2011 (PRN11), Kentucky. Planting dates in 2009 and 2010 were October 12 and October 14, respectively. The Lexington experiment was located at Spindletop Research Farm (387'37.81'’ N, 84²9'44.85'’W; Maury silt loam [fine, mixed, semiactive, mesic Typic Paleudalfs]) near Lexington, KY and the Princeton experiment was located at the West Kentucky Research and Educational Center (376' 7.37' ' N, 8752'13.62" W; Crider silt loam [fine-silty, mixed, active, mesic Typic Paleudalfs]). Liquid nitrogen fertilizer (28\% UAN) was applied in the spring at a rate of $105 \mathrm{~kg} \mathrm{~N} / \mathrm{ha}$ in split applications. Recommended agricultural practices for wheat production in Kentucky were followed (Lee et al., 2009).

\section{Phenotyping}

\section{Traditional methods}

Heading date, was recorded when $50 \%$ of the spikes in the row had emerged.

Plant height was measured at the soft dough stage. Foliar disease ratings and FHB ratings were also taken as described in chapter 3. Each plot was harvested with a mechanical combine for yield and test weight (TWT) determination. A 100-gram sample from each rep was analyzed for milling and baking quality at the SWQL, Wooster, OH. All grain was tempered at $15 \%$ moisture before milling. Whole grain protein and whole grain hardness were estimated using a NIR analyzer (DA7200, 950-1650 nm wavelength range). Flour yield was calculated as the bran weight subtracted from the grain weight, divided by the grain weight times 100 as described in Souza et al. (2008). Softness equivalent was calculated from the fraction of mill product that is in the mids that is subtracted from the adjusted flour yield. Flour protein was determined on flour using NIR (Unit Spectra-Star 2200, Columbia, MD) calibrated by nitrogen combustion analysis using Elementar Nitrogen Analyzer. Cookie diameter (ECD) was estimated in 2010 as 
described in Souza et al. (2011). Water, sucrose, sodium carbonate, and lactic acid SRC were estimated using approved AACC Method 56-11.02 (AACC, 2010).

\section{Wheat meal SDS sedimentation volume}

Wheat meal SDS was measured as described in Knott et al. (2009). Twenty five-g samples were milled with a Cyclone sample mill (UDY, Fort Collins, CO, 80524) using a 1-mm sieve. One evaluation per actual replicate was conducted each year. To dispense the solvents, deionized water, sodium lauryl sulfate and lactic acid, bottle top dispensers were utilized. As lactic acid solutions can degrade with time, the volume required was prepared fresh once a week. Eighteen samples were run per round, using three test tube rockers. Ten-ml of deionized water were dispensed into $25-\mathrm{mL}$ glass graduated cylinders. Wheat meal $(1 \mathrm{~g})$ was added to each cylinder, the cylinder was capped with a ground glass stopper, shaken vigorously for approximately $15 \mathrm{~s}$, and placed onto a test tube rocker to rest for $2 \mathrm{~min}$. After the rest period, the cylinders were inverted four times, allowed to rest for $2 \mathrm{~min}$, and inverted four times. Sodium lauryl sulfate $(10 \mathrm{~mL}, 2.5 \%$ $\mathrm{w} / \mathrm{v}$ ) was added to each cylinder and the cylinders were inverted four times and allowed to rest $2 \mathrm{~min}$. The procedure was repeated for a total of four cycles. Lactic acid $(5 \mathrm{~mL}$ of $1.1 \% \mathrm{w} / \mathrm{v}$ ) was dispensed into the cylinders. Four cycles of inverting the cylinders four times followed by a $2 \mathrm{~min}$ rest were completed. After the final inversion, the cylinders were removed from the rockers and allowed to settle for $20 \mathrm{~min}$ before sedimentation volume was measured.

\section{Wheat meal sodium carbonate SRC}

Wheat meal sodium carbonate SRC was conducted as described in Knott et al. (2009). Five-g wheat meal samples, produced as described above, were placed into disposable $50 \mathrm{~mL}$ centrifuge tubes and $25 \mathrm{~mL}$ of $5 \%(\mathrm{w} / \mathrm{w})$ sodium carbonate was added using a bottle-top dispenser. Twenty samples were run per round. The wheat meal was suspended into the sodium carbonate by shaking the tubes horizontally 40 times. Tubes were placed horizontally onto an orbital shaker and agitated for $20 \mathrm{~min}$ at $100 \mathrm{rpm}$. The tubes were centrifuged at $1000 \mathrm{x}$ g for $15 \mathrm{~min}$. The supernatant was decanted and the 
tubes were allowed to drain on absorbent towels for $10 \mathrm{~min}$. The tubes were weighed and solvent retention capacity was calculated as follows:

$$
\begin{aligned}
& \mathrm{SRC}=100 \times\{(\text { Pellet weight/Flour weight }) \times \\
& [86 /(100-\text { Wheat Meal Moisture })]-1\}
\end{aligned}
$$

\section{Near infrared reflectance}

Samples were run on a NIR analyzer (DA7200, 950-1650 nm wavelength range) manufactured by Perten Instruments (IL). A calibration built by the Ohio State University and the manufacturers was used to run 2010 samples and it was updated on the basis of wet chemistry 2010 data measured by traditional methods collected in this study to run 2011 samples. The amount of seed required is approximately $20 \mathrm{~g}$.

\section{Data analysis}

\section{Relationship between wheat meal and NIR based predictions with wet chemistry}

Proc CORR (SAS, 2002) was used to analyze the relationship between traits on an entry mean basis. Entry means were plotted using Microsoft Excel (2007) to study the relationship among traits and calculate $\mathrm{R}^{2}$.

\section{Heritability estimates}

Broad sense heritability of WM-SDS, WM-sodium carbonate SRC and NIR predictions were estimated on an entry mean basis using the following model:

$\mathrm{Y}_{\mathrm{ij}}=\mu+\mathrm{G}_{\mathrm{k}}+\mathrm{R}(\mathrm{ENV})_{\mathrm{ij}}+\mathrm{ENV}_{\mathrm{i}} * \mathrm{G}_{\mathrm{j}}+\mathrm{E}_{\mathrm{ij}}$

Where: $\mathrm{Y}_{\mathrm{ij}}=$ the observation in the $k$ th genotype in the $j$ th rep in the $i$ th environment, $\mu=$ the overall mean, $\mathrm{G}_{\mathrm{j}}=$ the effect of the $k$ th genotype, $\mathrm{R}(\mathrm{ENV})_{\mathrm{ij}}=$ the effect of $j$ th rep within $i$ th environment, $\mathrm{ENV}_{\mathrm{i}} * \mathrm{G}_{\mathrm{j}}=$ the effect of the interaction of the $i$ th environment with the $k$ th genotype, $\mathrm{E}_{\mathrm{ij}}=$ the residual error.

Data was analyzed using the General Linear Models procedure (Proc GLM; SAS 2002). Genotypic and phenotypic variances were estimated from the expected mean squares (EMS) and heritability estimates were computed as:

$\mathrm{h}^{2}=\mathrm{V}_{\mathrm{g}} / \mathrm{V}_{\mathrm{p}}$

where $\mathrm{h}^{2}=$ heritability, $\mathrm{V}_{\mathrm{g}}=$ genotypic variance, $\mathrm{V}_{\mathrm{p}}=$ phenotypic variance. 
Confidence intervals (90 \%) were calculated after Knapp et al. (1985) as:

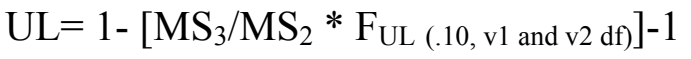

$\mathrm{LL}=1-\left[\mathrm{MS}_{3} / \mathrm{MS}_{2} * \mathrm{~F}_{\mathrm{LL}(.90, \mathrm{v} 1 \text { and } \mathrm{v} 2 \mathrm{df})]-1}\right.$

Where: $\mathrm{UL}=$ upper limit of the confidence interval, $\mathrm{MS}_{3}=$ entry mean square, $\mathrm{MS}_{2}=$ residual mean square, $\mathrm{F}_{\mathrm{UL}}$ and $\mathrm{F}_{\mathrm{LL}}=\mathrm{F}$ value for the upper and lower limits calculated using the FINV function of Microsoft Excel (2007).

\section{Selection simulation}

Selection was simulated to estimate genetic gain for various selection criteria using WM assays as quality predictors. LEX2010 was treated as the selection environment and PRN2011 was treated as the validation environment for milling and baking quality traits. The mean of the selected lines after one cycle of selection in 2011 was compared to the mean of the population in the absence of selection. On the basis of 2010 data, selection scenarios were simulated to compare the number of lines retained under direct selection against indirect selection. For example, the number of lines retained when the top $25 \%$ of the population with highest softness equivalent was compared against the number of lines retained when selecting for the top 25 and $50 \%$ of the population with highest softness equivalent measured using whole grain NIR.

\section{Results and discussion}

\section{Wheat meal assays}

Mean WM-SDS ranged from 7.4-9.9 and 7.0-8.2 in LEX2010 and PRN2011, respectively. Mean WM-sodium carbonate SRC ranged from 77.2-78.3 and 77.2-79.7 in LEX2010 and PRN2011, respectively (Table 4.1). All five populations showed maximum WM-SDS levels higher or close to the highest levels observed in the parents.

Trangressive segregates were also found for WM-sodium carbonate SRC in all the genetic backgrounds. These data suggests that selection for higher WM-SDS and lower WM-sodium carbonate SRC is possible in this background.

Genotype mean correlations were calculated between WM-SDS and WM-sodium carbonate SRC for baking and milling parameters (Table 4.2). Wheat meal SDS was 
moderately correlated with whole grain protein $(\mathrm{r}=0.43)$ and whole grain hardness $(\mathrm{r}=0.54)$, and strongly correlated with flour protein $(\mathrm{r}=0.67)$, flour lactic acid SRC $(\mathrm{r}=0.81)$ and GPI $(\mathrm{r}=0.80)$. Similar correlation coefficients were found between WM-SDS and flour lactic acid SRC (Gutierri et al., 2004). Correlation coefficients between WMSDS and flour protein reported by Gutierri et al. (2004) were not significant and 0.54, depending on the genetic background. Wheat meal sodium carbonate SRC showed a negative correlation with flour yield $(\mathrm{r}=-0.30)$ (Table 4.2) which is lower than the negative correlation 0.57-0.72 reported in three different genetic backgrounds (Gutierri et al., 2004) and it was positively and moderately correlated with all flour based SRC values (Table 4.2).

Flour yield was regressed on WM-sodium carbonate SRC by population to study the prediction ability of this test in the different genetic backgrounds. The relationship between this two traits was negative with the exception of population 2 that showed no relationship, and the $\mathrm{R}^{2}$ varied from 0-0.3 (Figure A.4.1 to A.4.5). The same analysis was conducted to evaluate the ability of WM-sodium carbonate SRC to predict flour sucrose $\mathrm{SRC}$ in the five different populations and $\mathrm{R}^{2}$ ranged from 0.68-0.16 (Figure A.4.6 to A.4.10). These wide ranges indicate that the usefulness of WM-sodium carbonate SRC varies with the genetic background in this study. Wheat meal SDS, on the other hand, was a very good predictor $\left(\mathrm{R}^{2}=0.53-0.87\right)$ of flour based lactic acid $\mathrm{SRC}$ in all five populations (Figure A.4.11 to A.4.15)

\section{Near infrared reflectance}

Whole grain and flour based NIR measurements taken at the SWQL were strongly correlated with those NIR measurements taken at UK. Whole grain protein and whole grain hardness measured in one lab and the other showed correlation coefficients of 0.97 and 0.89 , respectively (Table 4.3). Flour protein NIR predicted on a whole grain basis (UK) was highly correlated with flour protein NIR based on flour (SWQL) ( $\mathrm{r}=0.86)$. Whole grain NIR softness equivalent showed a moderate correlation with softness equivalent measured at the SWQL ( $\mathrm{r}=0.60)$. This relationship varied among populations

$\left(\mathrm{R}^{2}=0.17-0.49\right)$ (Figure A.4.16-A.4.20). In general, the ability of NIR to predict SRC was in insufficient. However, whole grain NIR water SRC was moderately correlated with 
flour water SRC and flour sodium carbonate SRC (0.49 and 0.50, respectively) (Table 4.3). The relationship between NIR water SRC and flour water SRC also varied among populations $\left(\mathrm{R}^{2}=0.06-0.59\right)$ (Figure A.4.21 to A.4.25). These results indicate that for some quality parameters like whole grain protein, flour protein and hardness, whole grain NIR is a valuable tool for use in soft red winter wheat breeding programs. Although with more caution, NIR could be also used to enrich earlier generations with desirable softness equivalent, flour yield and flour water SRC. This aspect will be discussed in the selection simulation section.

\section{Selection simulation}

\section{Wheat meal assays}

Broad sense $\mathrm{h}^{2}$ and their corresponding confidence intervals were estimated on an entry mean basis for each population separately as described earlier (Table 4.4). Heritability estimates were moderate to high and ranged from 0.70 to 0.94 and 0.60 to 0.92 for WM-SDS and WM sodium carbonate SRC, respectively. Knott et al. (2009) estimated 0.70 and 0.67 for WM-SDS and WM-sodium carbonate SRC broad sense $\mathrm{h}^{2}$. Although WM based $\mathrm{h}^{2}$ estimates were lower than the flour based SRC and flour yield, they were consistently high which suggests their utility in selecting for milling and baking quality.

A selection scenario was simulated by first retaining lines with desirable agronomic characteristics. Short early genotypes $(<93 \mathrm{~cm}$ tall, $\leq 130$ Julian days heading date) with $\geq 70.8 \mathrm{~kg} / \mathrm{hl}$ TWT were considered acceptable. Next, selection pressure was simulated at $\mathrm{p}=0.25$ and 0.12 . At either selection pressure for highest WM-SDS, the response was slightly lower than when the selection was conducted directly on lactic acid SRC (Table 4.5a). These results indicate that WM-SDS, a much more rapid and easy assay than the flour lactic acid SRC, can be effectively used to select for higher gluten strength lines within SRW wheat populations. At 25 and 50\% WM-SDS selection pressure, the proportion of lines that would have been selected using flour lactic acid SRC directly $(\mathrm{p}=0.25)$ were 58 and 90\%, respectively (Table 4.6). Mean lactic acid SRC of the selected population based on WM-SDS is very close to the mean of the selected population based on flour lactic acid SRC directly (101.3 and 102.6\%, respectively) 
which are higher than the $94 \%$ of the initial population (Table 4.6). These results provide more evidence of the power of WM-SDS as a selection criterion for higher gluten strength.

Another selection simulation scenario was conducted by retaining the $12 \%$ of the lines with lowest WM-sodium carbonate SRC. Response to selection was very low $(2.3 \%$ reduction) but direct selection on flour sodium carbonate SRC was also low (3.2\% reduction). Indirect response on flour yield and sucrose was inexistent when selection was based on flour sodium carbonate SRC, and very small when selection was based on the wheat meal test (Table $4.5 \mathrm{~b}$ ). In contrast, a 12\% direct selection pressure for higher flower yield resulted in a 4\% increase in 2011 (data not shown). At 25 and 50\% WMsodium carbonate SRC selection pressure, the proportion of lines that would have been selected using flour yield directly $(\mathrm{p}=0.25)$ were 35 and 65\%, respectively (Table 4.7). Once again, even though WM-sodium carbonate SRC did not identify all of the lines with highest flour yield, a 50\% selection pressure would enrich early generations with lines with higher flour yield at stages where lines are not normally evaluated for this trait. The small difference between mean flour yield before selection and after a simulated $p=25$ selection pressure may indicate a small potential for response to selection in this particular set of populations (Table 4.7).

\section{Near infrared reflectance}

As described above, an initial selection scenario was simulated by first retaining lines with desirable agronomic characteristics. From this subpopulation, only the top 25\% lines with higher softness equivalent were selected based on softness equivalent directly and based on NIR softness equivalent (Table 4.8). Indirect selection on softness equivalent using NIR identified 15 of the 31 lines that were selected directly. Reducing the selection pressure to $50 \%$ allowed 22 of the 31 lines to be identified. Although selection based on NIR softness equivalent did not identify all of the lines that could have been kept in the direct selection scenario, this tool is valuable in the early stages of a wheat breeding program when softness equivalent is not normally measured. A possible strategy could be to use a lower selection pressure $(p=0.50)$ to reduce the chances of discarding lines with desirable softness equivalent levels. Response to simulated direct 
and NIR based selection ( $\mathrm{p}=0.12$ ) on softness equivalent was $8 \%$ and $2 \%$, respectively (data not shown). However, even at the lowest selection pressure $(p=0.50)$ the mean softness equivalent of the selected population is 1 point higher than the mean of the initial population (Table 4.8).

A similar analysis was conducted using NIR as flour yield and flour water SRC predictor. When using whole grain NIR flour yield at a 25 and 50\% selection pressure, the proportion of lines that would have retained using flour yield directly $(p=0.25)$ were 26 and 48\%, respectively (Table 4.9). Response to simulated direct and NIR based selection ( $\mathrm{p}=0.12$ ) was 4 and $1.2 \%$, respectively (data not shown). As mentioned above in the flour yield selection scenario based on WM-sodium carbonate SRC, the small difference among mean flour yield in the initial population and in the selected lines with either criterion suggest a small response potential in these populations.

When using whole grain NIR water SRC at a 25 and 50\% selection pressure, the proportion of lines that would have been retained using flour water SRC $(p=0.25)$ were 39 and 58\%, respectively (Table 4.10). However, mean flour water SRC in the selected population using NIR at either selection pressure was very close to the mean of the initial population. Response to simulated direct selection $(\mathrm{p}=0.12)$ was low $(3.2 \%)$ and when NIR based selection was imparted, there was no response reduction in water SRC (data not shown). More adjustments may need to be done to use whole grain NIR measurements of flour yield and flour water SRC. However, moderate correlations between NIR and these two parameters suggest that there is potential of calibration improvements to achieve more accurate predictions.

\section{Conclusions}

Wheat meal SDS predicted flour lactic acid SRC reasonably well in these diverse genetic backgrounds $(\mathrm{r}=0.81)$. Although the response for selection to higher flour lactic acid SRC based on WM-SDS was small $(7.6 \%$ at $\mathrm{p}=0.12)$, this was close to the response obtained when selection was made directly on the flour based parameter $(8.9 \%$ at $\mathrm{p}=0.12$ ). In this study, wheat meal sodium carbonate SRC showed a lower predictive value of flower yield than expected $(\mathrm{r}=-0.30)$ and was less useful for selection purposes. 
Wheat meal SDS and WM-sodium carbonate SRC effectively identified a high proportion of the lines that would have been identified using direct flour measurements. Results obtained in this study indicate that whole grain NIR is a valuable tool to predict whole grain protein, hardness and flour protein. For example, NIR based selections for flour softness equivalent at a lower selection pressure $(\mathrm{p}=0.50)$ retained $71 \%$ of the lines that would have been retained at $\mathrm{p}=0.25$ direct selection on flour yield and could be successfully used to enrich breeding populations with higher softness equivalent. Although some adjustments need to be done before using NIR to predict other quality parameters, moderate correlations found for flour yield and flour water SRC suggest that there is potential for calibrations improvement. There are a number of important advantages to continue the work on improving the NIR instrument calibration for quality predictions: (i) no milling is required, it saves time and is non-destructive, (ii) it requires a small amount of seed (20-grams), (iii) it is rapid and simple to operate, (iv) with the appropriate calibration, a number of parameters can be estimated at the same time, and (v) it is objective. 
Table 4.1: Mean, Maximum (Max) and Minimum (Min) wheat meal SDS sedimentation (WM-SDS) and wheat meal sodium carbonate SRC (WM-SCSRC) for five wheat populations and their parents in Lexington (LEX) 2010 and Princeton (PRN) 2011.

\begin{tabular}{|c|c|c|c|c|c|c|c|c|c|}
\hline \multirow{3}{*}{$\mathbf{N}$} & & \multicolumn{4}{|c|}{ LEX 2010} & \multicolumn{4}{|c|}{ PRN 2011} \\
\hline & & WM-SDS & & \multicolumn{2}{|c|}{ WM-SCSRC } & \multirow{2}{*}{\multicolumn{2}{|c|}{$\begin{array}{c}\text { WM-SDS } \\
\text { (ml) }\end{array}$}} & \multirow{2}{*}{\multicolumn{2}{|c|}{$\begin{array}{c}\text { WM-SCSRC } \\
(\%) \\
\end{array}$}} \\
\hline & & $(\mathrm{ml})$ & & \multicolumn{2}{|l|}{$(\%)$} & & & & \\
\hline \multirow{2}{*}{$\mathbf{N}$} & \multicolumn{9}{|c|}{ Population 1 (26R58/VA01W-476//KY97C-0574-01) } \\
\hline & Mean & 7.9 & & 77.2 & & 7.7 & & 77.2 & \\
\hline \multirow[t]{4}{*}{21} & Max & 10.5 & & 81.6 & & 10.0 & & 83.5 & \\
\hline & Min & 5.5 & & 73.3 & & 5.5 & & 71.3 & \\
\hline & \multicolumn{9}{|c|}{ Population 2 (25R54/VA01W-476//KY97C-0574-01) } \\
\hline & Mean & 8.3 & & 78.3 & & 7.7 & & 76.9 & \\
\hline \multirow[t]{4}{*}{24} & Max & 10.5 & & 84.9 & & 10.0 & & 85.6 & \\
\hline & Min & 5.5 & & 74.1 & & 5.0 & & 71.7 & \\
\hline & \multicolumn{9}{|c|}{ Population 3 (25R54/VA01W-476//KY97C-0554-02) } \\
\hline & Mean & 9.9 & & 77.4 & & 8.2 & & 78.7 & \\
\hline \multirow[t]{4}{*}{36} & Max & 14.0 & & 88.7 & & 12.0 & & 92.5 & \\
\hline & Min & 6.0 & & 71.3 & & 6.0 & & 73.4 & \\
\hline & \multicolumn{9}{|c|}{ Population 4 (25R78/VA01W-476) } \\
\hline & Mean & 8.9 & & 77.5 & & 7.9 & & 79.7 & \\
\hline \multirow[t]{4}{*}{45} & Max & 12.0 & & 83.3 & & 12.5 & & 88.5 & \\
\hline & Min & 5.5 & & 73.1 & & 5.0 & & 74.6 & \\
\hline & \multicolumn{9}{|c|}{ Population 5 (KY93C-1238-17-1/VA01W-476) } \\
\hline & Mean & 7.4 & & 77.5 & & 7.0 & & 78.1 & \\
\hline \multirow[t]{9}{*}{29} & Max & 10.0 & & 81.4 & & 9.0 & & 83.2 & \\
\hline & Min & 5.5 & & 74.4 & & 5.0 & & 74.3 & \\
\hline & $25 \mathrm{R} 54$ & 7.1 & $(0.3)$ & 77.3 & $(0.4)$ & 6.9 & $(0.4)$ & 76.4 & $(0.6)$ \\
\hline & $25 \mathrm{R} 78$ & 7.7 & $(0.5)$ & 75.4 & $(0.7)$ & 7.5 & $(0.6)$ & 78.1 & $(0.8)$ \\
\hline & 26R58 & 6.3 & $(0.5)$ & 76.2 & $(0.7)$ & 6.5 & $(0.6)$ & 76.5 & $(0.8)$ \\
\hline & KY93C-1238-17-1 & 4.3 & $(0.5)$ & 77.2 & $(0.7)$ & 4.3 & $(0.6)$ & 77.2 & $(0.8)$ \\
\hline & KY97C-0554-02 & 8.8 & $(0.5)$ & 78.6 & $(0.7)$ & 9.0 & $(0.6)$ & 81.8 & $(0.8)$ \\
\hline & KY97C-0574-01 & 8.3 & (0.4) & 77.1 & $0.5)$ & 7.9 & $(0.4)$ & 76.4 & (0.6) \\
\hline & VA01W-476 & 10.2 & $(0.3)$ & 80.1 & $(0.3)$ & 8.5 & $(0.3)$ & 81.0 & (0.4) \\
\hline
\end{tabular}

Numbers in parentheses are standard errors (SE) 
Table 4.2: Genotype mean correlations for whole grain protein, whole grain hardness, flour yield, softness equivalent, flour protein, flour solvent retention capacity profile (SRC), gluten performance index (GPI) and estimated cookie diameter of 155 wheat lines with wheat meal SDS sedimentation (WM-SDS) and WM-sodium carbonate SRC in Lexington 2010 and Princeton 2011.

\begin{tabular}{|c|c|c|}
\hline & WM-SDS & $\begin{array}{l}\text { WM-sodium } \\
\text { carbonate SRC }\end{array}$ \\
\hline \multirow{2}{*}{ Whole grain protein } & 0.43 & -0.17 \\
\hline & $<.0001$ & 0.0365 \\
\hline \multirow{2}{*}{ Whole grain hardness } & 0.54 & 0.26 \\
\hline & $<.0001$ & 0.0009 \\
\hline \multirow{2}{*}{ Flour yield } & 0.06 & -0.30 \\
\hline & 0.4783 & 0.0002 \\
\hline \multirow{2}{*}{ Softness equivaent } & -0.38 & 0.23 \\
\hline & $<.0001$ & 0.004 \\
\hline \multirow{2}{*}{ Flour protein } & 0.67 & -0.05 \\
\hline & $<.0001$ & 0.5098 \\
\hline \multirow{2}{*}{ Water SRC } & 0.29 & 0.46 \\
\hline & 0.0002 & $<.0001$ \\
\hline \multirow{2}{*}{ Lactic acid SRC } & 0.81 & 0.42 \\
\hline & $<.0001$ & $<.0001$ \\
\hline \multirow{2}{*}{ Sucrose SRC } & 0.05 & 0.48 \\
\hline & 0.5111 & $<.0001$ \\
\hline \multirow{2}{*}{ Sodium carbonate SRC } & 0.17 & 0.62 \\
\hline & 0.0388 & $<.0001$ \\
\hline \multirow{2}{*}{ GPI } & 0.80 & 0.24 \\
\hline & $<.0001$ & 0.0027 \\
\hline \multirow{2}{*}{ Estimated cookie diameter } & -0.37 & -0.03 \\
\hline & $<.0001$ & 0.6731 \\
\hline
\end{tabular}

The upper number for each trait is the Pearson correlation coefficient and the number below is the $\mathrm{P}$ value. 
Table 4.3: Genotype mean correlation for whole grain NIR predictions and flour and wheat meal based quality parameters in Lexington 2010 and Princeton 2011.

\begin{tabular}{|c|c|c|c|c|c|c|c|c|c|c|}
\hline & & \multicolumn{9}{|c|}{ UK-whole grain NIR } \\
\hline & & $\begin{array}{c}\text { Whole grain } \\
\text { protein }\end{array}$ & $\begin{array}{l}\text { Whole grain } \\
\text { hardness }\end{array}$ & $\begin{array}{l}\text { Softness } \\
\text { equivalent }\end{array}$ & $\begin{array}{l}\text { Flour } \\
\text { yield }\end{array}$ & $\begin{array}{l}\text { Flour } \\
\text { protein }\end{array}$ & $\begin{array}{l}\text { Water } \\
\text { SRC }\end{array}$ & $\begin{array}{c}\text { Sodium } \\
\text { carbonate } \\
\text { SRC }\end{array}$ & $\begin{array}{c}\text { Sucrose } \\
\text { SRC }\end{array}$ & $\begin{array}{c}\text { Lactic acid } \\
\text { SRC }\end{array}$ \\
\hline \multirow{4}{*}{ 占 } & \multirow{2}{*}{ WM-SDS } & 0.45 & 0.60 & -0.36 & -0.40 & 0.52 & 0.32 & 0.33 & 0.26 & 0.23 \\
\hline & & $<.0001$ & $<.0001$ & $<.0001$ & $<.0001$ & $<.0001$ & $<.0001$ & $<.0001$ & 0.0012 & 0.0035 \\
\hline & \multirow{2}{*}{ WM-sodium carbinate SRC } & -0.17 & 0.26 & 0.24 & -0.39 & -0.10 & 0.41 & 0.50 & 0.23 & -0.12 \\
\hline & & 0.0353 & 0.0013 & 0.0027 & $<.0001$ & 0.2341 & $<.0001$ & $<.0001$ & 0.0046 & 0.1457 \\
\hline \multirow{8}{*}{$\sum_{\infty}^{0}$} & \multirow{2}{*}{ Whole grain protein (SWQL NIR) } & 0.97 & 0.42 & -0.55 & -0.29 & 0.94 & -0.01 & 0.00 & 0.26 & 0.26 \\
\hline & & $<.0001$ & $<.0001$ & $<.0001$ & 0.0002 & $<.0001$ & 0.9206 & 0.9774 & 0.0009 & 0.001 \\
\hline & \multirow{2}{*}{ Whole grain hardness (SWQL NIR) } & 0.41 & 0.89 & -0.35 & -0.69 & 0.55 & 0.34 & 0.38 & 0.40 & 0.08 \\
\hline & & $<.0001$ & $<.0001$ & $<.0001$ & $<.0001$ & $<.0001$ & $<.0001$ & $<.0001$ & $<.0001$ & 0.3393 \\
\hline & \multirow{2}{*}{ Flour yield } & -0.14 & -0.03 & -0.28 & 0.28 & -0.13 & 0.09 & 0.05 & -0.21 & 0.27 \\
\hline & & 0.0824 & 0.6834 & 0.0005 & 0.0004 & 0.1007 & 0.2826 & 0.5509 & 0.0085 & 0.0006 \\
\hline & \multirow{2}{*}{ Softness equivalent } & -0.41 & -0.38 & 0.60 & -0.08 & -0.44 & -0.30 & 0.04 & 0.06 & 0.03 \\
\hline & & $<.0001$ & $<.0001$ & $<.0001$ & 0.3451 & $<.0001$ & 0.0002 & 0.5925 & 0.4827 & 0.7045 \\
\hline
\end{tabular}

The upper number for each trait is the Pearson correlation coefficient and the number below is the $\mathrm{P}$ value. 
Table 4.3 (continued): Genotype mean correlation for whole grain NIR predictions and flour and wheat meal based quality parameters in Lexington 2010 and Princeton 2011.

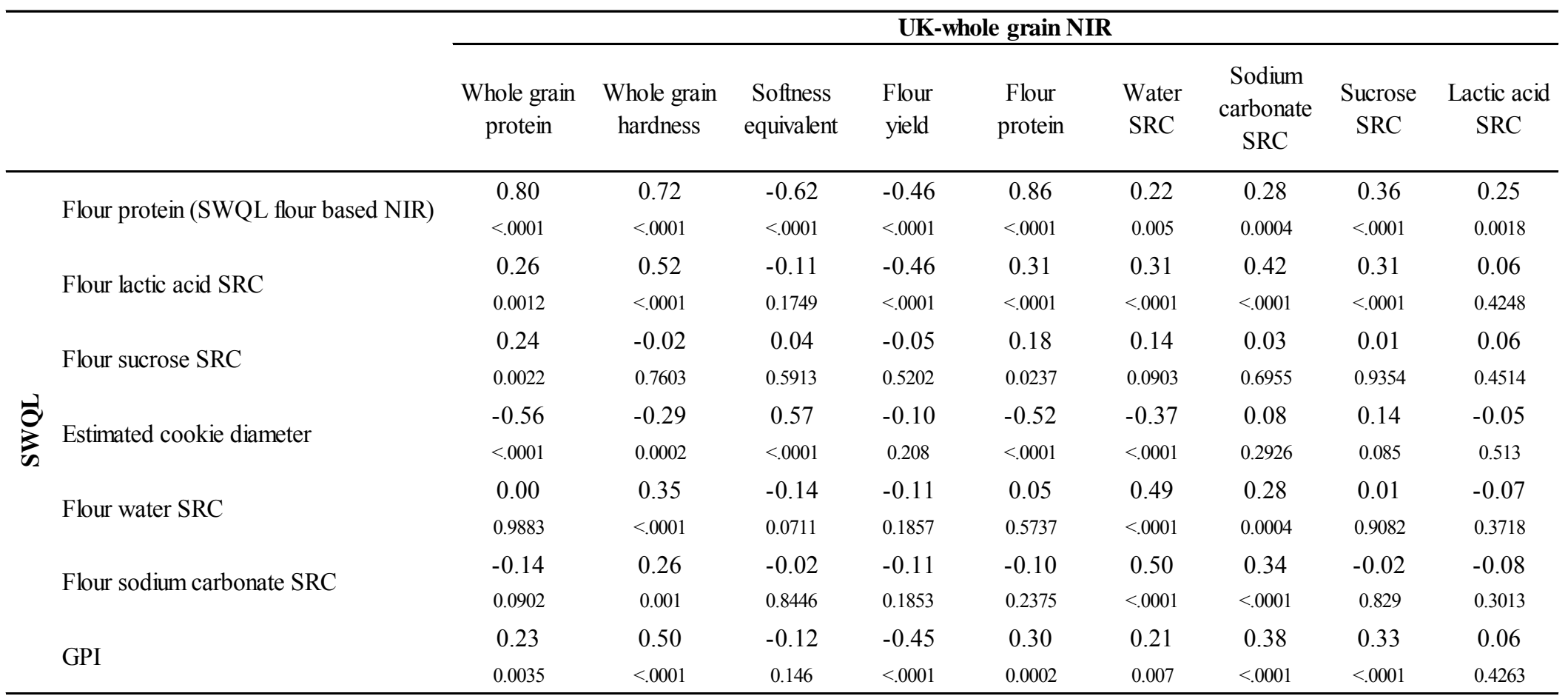

The upper number for each trait is the Pearson correlation coefficient and the number below is the $\mathrm{P}$ value. 
Table 4.4: Wheat meal sedimentation (WM-SDS), WM-sodium carbonate SRC, flour lactic acid SRC, flour sodium carbonate SRC and flour yield heritabilities and their 90\% confidence intervals in parentheses, based on a 2 year ANOVA of five wheat populations evaluated in Lexington 2010 and Princeton 2011.

\begin{tabular}{cccccccccccc}
\hline & \multicolumn{2}{c}{ WM-SDS } & \multicolumn{2}{c}{$\begin{array}{c}\text { WM-sodium } \\
\text { carbonate SRC }\end{array}$} & \multicolumn{2}{c}{$\begin{array}{c}\text { Flour lactic } \\
\text { acid SRC }\end{array}$} & \multicolumn{2}{c}{$\begin{array}{c}\text { Flour sodium } \\
\text { carbonate SRC }\end{array}$} & \multicolumn{3}{c}{ Flour yield } \\
\hline Overall & 0.83 & $(0.79-0.86)$ & 0.82 & $(0.85-0.77)$ & 0.95 & $(0.96-0.93)$ & 0.84 & $(0.87-0.80)$ & 0.94 & $(0.95-0.92)$ \\
Population 1 & 0.75 & $(0.55-0.86)$ & 0.78 & $(0.60-0.88)$ & 0.93 & $(0.96-0.87)$ & 0.84 & $(0.91-0.71)$ & 0.85 & $(0.92-0.74)$ \\
Population 2 & 0.94 & $(0.89-0.96)$ & 0.94 & $(0.90-0.97)$ & 0.95 & $(0.97-0.92)$ & 0.75 & $(0.85-0.56)$ & 0.90 & $(0.94-0.84)$ \\
Population 3 & 0.85 & $(0.77-0.91)$ & 0.89 & $(0.82-0.93)$ & 0.96 & $(0.97-0.94)$ & 0.93 & $(0.96-0.90)$ & 0.95 & $(0.97-0.93)$ \\
Population 4 & 0.83 & $(0.75-0.88)$ & 0.92 & $(0.88-0.94)$ & 0.82 & $(0.88-0.74)$ & 0.89 & $(0.93-0.84)$ & 0.88 & $(0.92-0.82)$ \\
Population 5 & 0.70 & $(0.51-0.82)$ & 0.60 & $(0.34-0.75)$ & 0.89 & $(0.93-0.82)$ & 0.85 & $(0.91-0.75)$ & 0.93 & $(0.96-0.88)$ \\
\hline
\end{tabular}


Table 4.5: Mean flour lactic acid SRC increment after one cycle of direct and indirect simulated selection, proportion of the population selected (p) and number of lines selected (a), and mean flour sodium carbonate SRC, flour yield and flour sucrose SRC reduction after one cycle of direct and indirect simulated selection, proportion of the population selected and number of lines selected (b)

(a)

\begin{tabular}{lccc}
\hline Selection criterion & p & n & $\begin{array}{c}\text { Flour lactic acid SRC } \\
(\%)\end{array}$ \\
\hline Direct phenotypic selection (Flour lactic acid SRC) & \multirow{2}{*}{0.25} & 31 & 7.1 \\
Indirect phenotypic selection (WM-SDS) & & & 5.3 \\
Direct phenotypic selection (Flour lactic acid SRC) & \multirow{2}{*}{0.12} & 15 & 8.9 \\
Indirect phenotypic selection (WM-SDS) & & & 7.6 \\
\hline
\end{tabular}

(b)

\begin{tabular}{lccccc}
\hline Selection criterion & p & n & Flour sodium carbonate SRC & $\begin{array}{c}\text { Flour yield } \\
\text { Flour sucrose SRC }\end{array}$ \\
\hline Direct phenotypic selection (Flour sodium carbonate SRC) & \multirow{2}{*}{0.12} & \multirow{2}{*}{15} & -3.2 & 0 & 0 \\
Indirect phenotypic selection (WM-sodium carbonate SRC) & & & -2.3 & 0.9 & -2.2 \\
\hline
\end{tabular}


Table 4.6: Number of lines retained when the highest 50 and $25 \%$ of the population were selected for gluten strength based on wheat meal sedimentation (WM-SDS) as compared with the number of lines retained when the highest $25 \%$ of the population was selected based on flour lactic acid solvent retention capacity (SRC).

\begin{tabular}{|c|c|c|c|c|}
\hline Selection criterion & $\begin{array}{l}\text { No of lines } \\
\text { retained }\end{array}$ & $\begin{array}{l}\text { Mean flour lactic acid SRC } \\
\text { of lines retained (\%) }\end{array}$ & $\begin{array}{l}\text { No. of lines identified that were } \\
\text { selected under direct } 25 \% \\
\text { flour lactic acid SRC selection }\end{array}$ & Percentage \\
\hline None & 155 & 94.1 & 0 & 0 \\
\hline $\begin{array}{l}\text { Agronomic performance } \\
\text { (30 lines discarded) }\end{array}$ & 125 & 94.0 & 0 & 0 \\
\hline $\begin{array}{l}\text { Agronomic performance } \\
\text { then } 25 \% \text { lactic acid SRC }\end{array}$ & 31 & 102.6 & 31 & 100 \\
\hline $\begin{array}{l}\text { Agronomic performance } \\
\text { then } 25 \% \text { WM-SDS }\end{array}$ & 31 & 101.3 & 18 & 58 \\
\hline $\begin{array}{l}\text { Agronomic performance } \\
\text { then } 50 \% \text { WM-SDS }\end{array}$ & 62 & 98.8 & 28 & 90 \\
\hline
\end{tabular}


Table 4.7: Number of lines retained when the highest 50 and $25 \%$ of the population were selected for flour yield based on the lowest wheat meal (WM) sodium carbonate solvent retention capacity (SRC) as compared with the number of lines retained when the highest $25 \%$ of the population was selected based on flour yield.

\begin{tabular}{|c|c|c|c|c|}
\hline Selection criterion & $\begin{array}{l}\text { No of lines } \\
\text { retained }\end{array}$ & $\begin{array}{c}\text { Mean flour yield } \\
\text { of lines retained (\%) }\end{array}$ & $\begin{array}{l}\text { No. of lines identified that were } \\
\text { selected under direct } 25 \% \\
\text { flour yield selection }\end{array}$ & Percentage \\
\hline None & 155 & 67.7 & 0 & 0 \\
\hline $\begin{array}{l}\text { Agronomic performance } \\
\text { (30 lines discarded) }\end{array}$ & 125 & 67.8 & 0 & 0 \\
\hline $\begin{array}{l}\text { Agronomic performance } \\
\text { then } 25 \% \text { flour yield }\end{array}$ & 31 & 69.9 & 31 & 100 \\
\hline $\begin{array}{l}\text { Agronomic performance } \\
\text { then } 25 \% \mathrm{WM} \text {-sodium carbonate SRC }\end{array}$ & 31 & 68.4 & 11 & 35 \\
\hline $\begin{array}{l}\text { Agronomic performance } \\
\text { then } 50 \% \text { WM-sodium carbonate SRC }\end{array}$ & 62 & 68.2 & 20 & 65 \\
\hline
\end{tabular}


Table 4.8: Number of lines retained when the highest 50 and $25 \%$ of the population were selected for softness equivalent based on whole grain NIR softness equivalent as compared with the number of lines retained when the highest $25 \%$ of the population was selected based on softness equivalent.

\begin{tabular}{lcccc}
\hline \multicolumn{1}{c}{ Selection criterion } & $\begin{array}{c}\text { No of lines } \\
\text { retained }\end{array}$ & $\begin{array}{c}\text { Mean softness equivalent } \\
\text { of lines retained (\%) }\end{array}$ & $\begin{array}{c}\text { No. of lines identified that were } \\
\text { selected under direct 25\% } \\
\text { softness equivalent selection }\end{array}$ & $\begin{array}{c}\text { Percentage } \\
\text { None }\end{array}$ \\
\hline $\begin{array}{l}\text { Agronomic performance } \\
\text { (30 lines discarded) }\end{array}$ & 155 & 56.2 & 0 & 0 \\
$\begin{array}{l}\text { Agronomic performance } \\
\text { then 25\% softness equivalent }\end{array}$ & 31 & 55.9 & 31 & 100 \\
$\begin{array}{l}\text { Agronomic performance } \\
\text { then 25\% NIR softness equivalent }\end{array}$ & 31 & 60.5 & 15 & 48 \\
$\begin{array}{l}\text { Agronomic performance } \\
\text { then 50\% NIR softness equivalent }\end{array}$ & 62 & 57.9 & 22 & 71 \\
\hline
\end{tabular}


Table 4.9: Number of lines retained when the highest 50 and $25 \%$ of the population were selected for flour yield based on whole grain NIR flour yield as compared with the number of lines retained when the highest $25 \%$ of the population was selected based on flour yield.

\begin{tabular}{|c|c|c|c|c|}
\hline Selection criterion & $\begin{array}{l}\text { No of lines } \\
\text { retained }\end{array}$ & $\begin{array}{l}\text { Mean flour yield of } \\
\text { lines retained (\%) }\end{array}$ & $\begin{array}{l}\text { No. of lines identified that were } \\
\text { selected under direct } 25 \% \\
\text { flour yield selection }\end{array}$ & Percentage \\
\hline None & 155 & 67.7 & 0 & 0 \\
\hline $\begin{array}{l}\text { Agronomic performance } \\
\text { (30 lines discarded) }\end{array}$ & 125 & 67.8 & 0 & 0 \\
\hline $\begin{array}{l}\text { Agronomic performance } \\
\text { then } 25 \% \text { flour yield }\end{array}$ & 31 & 69.9 & 31 & 100 \\
\hline $\begin{array}{l}\text { Agronomic performance } \\
\text { then } 25 \% \text { NIR flour yield }\end{array}$ & 31 & 67.8 & 8 & 26 \\
\hline $\begin{array}{l}\text { Agronomic performance } \\
\text { then } 50 \% \text { NIR flour yield }\end{array}$ & 62 & 68.2 & 15 & 48 \\
\hline
\end{tabular}


Table 4.10: Number of lines retained when the lowest 50 and $25 \%$ of the population were selected for flour water solvent retention capacity (SRC) based on whole grain NIR water SRC as compared with the number of lines retained when the lowest $25 \%$ of the population was selected based on flour water SRC.

\begin{tabular}{|c|c|c|c|c|}
\hline Selection criterion & $\begin{array}{l}\text { No of lines } \\
\text { retained }\end{array}$ & $\begin{array}{c}\text { Mean flour water SRC } \\
\text { of lines retained (\%) }\end{array}$ & $\begin{array}{l}\text { No. of lines identified that were } \\
\text { selected under direct } 25 \% \\
\text { flour water SRC selection }\end{array}$ & Percentage \\
\hline None & 155 & 54.4 & 0 & 0 \\
\hline $\begin{array}{l}\text { Agronomic performance } \\
\text { (30 lines discarded) }\end{array}$ & 125 & 54.3 & 0 & 0 \\
\hline $\begin{array}{l}\text { Agronomic performance } \\
\text { then } 25 \% \text { water SRC }\end{array}$ & 31 & 52.3 & 31 & 100 \\
\hline $\begin{array}{l}\text { Agronomic performance } \\
\text { then } 25 \% \text { NIR water SRC }\end{array}$ & 31 & 53.8 & 12 & 39 \\
\hline $\begin{array}{l}\text { Agronomic performance } \\
\text { then } 50 \% \text { NIR water SRC }\end{array}$ & 62 & 54.0 & 18 & 58 \\
\hline
\end{tabular}




\section{Chapter Five}

Prediction of FDK and DON using near infrared reflectance

\section{Introduction}

The kernel traits most indicative of head scab damage are Fusarium damaged kernels (FDK) and DON. Common methods to measure FDK involve quantifying the proportion of unhealthy or scabby grains in a random sample of grain. The most direct but most time-consuming methodology consists in taking a random subsample of 200 grains or more, sorting scabby and healthy grains and expressing the number of scabby grains over the total number of grains (Verges et al., 2006). Visual ratings on a 1-9 scale are also common, where 1 equals absence and 9 equals $100 \%$ of scabby kernels. Although visual assessments can be conducted more rapidly, this technique is highly subjective. More rapid and objective methods consist in separating healthy and sound fractions by weight using a gravity separation device such as a modified Precision Machine head thresher (Precision Machine Company, Inc., Lincoln, NE) (Knott, 2007) or an air separation machine developed from a Precision Machine head thresher and a ShopVac vacuum (Agostinelli, 2008; Agostinelli et al., 2012). With either method, the proportion of FDK is expressed on a weight basis (grams of damaged kernels/grams of total sample). The air separation method takes approximately 1 min per sample. A possible weakness of this approach is that symptomatic kernels are frequently lighter so the proportion of FDK expressed on a weight basis may be lower than the proportion expressed on a number basis. For example, in a 200 -grain sample, what would be a $50 \%$ FDK will probably be lower expressed in $\mathrm{g} / \mathrm{g}$ than in number/number. There are other factors, such as physiological stress during grain filling, foliar or non-scab spike diseases, which may result in shriveled kernels. Air separation measurements do not account for these factors and may lead to overestimations of FDK. DON is most commonly determined by gas chromatography with mass spectrometry (GC-MS) (Mirocha et al., 1998). There are ELISA-based vomitoxin kits available such as the EZ-Quant ${ }^{\mathrm{TM}}$ Vomitoxin Test Kit (Diagnostix, Mississauga, Ontario, Canada). 
Both DON and FDK are expensive and time consuming to quantify; thus, rapid and non-destructive methods for predicting these traits are of great interest. Near infrared (NIR) reflectance detects the absorption response by overtone and combination frequencies of O-H, C-H, and N-H molecular vibrations (Approved Method 39-00.01, 39-25.01, AACC, 2011) as well as physical properties like grain shape, size and color (Peiris et al, 2010). With the appropriate calibration an NIR instrument can measure FDK and DON on a whole kernel basis at the same time and 100 samples can be run in 3 hours. A calibration built in 2007 by the University of Kentucky Wheat Breeding Program and the manufacturers showed strong positive correlations between FDK and DON measured with traditional methods and NIR in two-way crosses. Coefficients of determination $\left(\mathrm{R}^{2}\right)$ of FDK measured using NIR, and FDK and DON measured by traditional methods, were 0.67 and 0.59 , respectively. This NIR calibration also predicted DON very well $\left(\mathrm{R}^{2}\right.$ DON-NIRDON $\left.=0.68\right)($ Agostinelli, unpublished data).

The objectives of this study were (i) to evaluate the use of NIR to predict FDK and DON and (ii) to study the relationship between NIR measurements and other FHB traits.

\section{Materials and methods}

\section{Plant material}

Five sets of inbred lines derived from 2 and 3-way crosses were evaluated in this study. Crosses were made between FHB susceptible parents (26R58, KY97C-0574-01, 25R54, KY97C, KY97C-0554-02, 25R78 and KY93C-1238-17-1) and FHB-resistant VA01W-476, a doubled haploid line derived from the cross 'Roane'/W14. These crosses represent typical resistant $\mathrm{x}$ susceptible crosses used in SRW wheat breeding programs. Lines comprising each population were chosen from an initial group of 27 crosses genotyped at the Regional Small Grains Genotyping lab (RSGGL) in Raleigh, NC (http://www.ars.usda.gov/saa/psru, verified 2/22/11) in 2007. $\mathrm{F}_{2}$ progeny were evaluated for the presence of resistance alleles at Fhbl and QFhs.nau-2DL. Markers used were Xgwm533 (Röder et al., 1998) for Fhb1, and Xcfd233 (Grain genes 2.0 at http://wheat.pw.usda.gov/GG2/index.shtml, verified 02/13/09) for QFhs.nau-2DL. Ten 
heads from each $\mathrm{F}_{2: 3}$ head row were threshed in bulk in 2008 to provide seed for this study.

\section{Lexington scab nursery}

To evaluate $\mathrm{FHB}$ traits, $\mathrm{F}_{2: 5}$ and $\mathrm{F}_{2: 6}$ lines were planted in headrows on 12 October 2009 and 20 October 2010 in a scab nursery at Spindletop Research Farm (38’37.81’’ N, 84²9'44.85' 'W; Maury silt loam [fine, mixed, semiactive, mesic Typic Paleudalfs]) near Lexington, KY (LEX). Lines were planted in rows $1.2 \mathrm{~m}$ long, spaced $30 \mathrm{~cm}$ apart. To provide favorable conditions for the disease, rows were misted with an overhead mist irrigation system on an automatic timer. Mist irrigation was on from May 11 to June 16, for periods of 5 minutes, every quarter hour from $8 \mathrm{pm}$ to 8:45, $11 \mathrm{pm}$ to 11:45 pm, 2:00 to 2:45 am, 5 to 5:30 am and $8.30 \mathrm{am}$ (e.g. the equipment operated from $8 \mathrm{pm}$ to 8:05 pm the first time and the last time in the misting cycle was from 8:30 am to 8:35 am). The nursery was inoculated with Fusarium graminearum-infected corn (Zea mays L.) (Verges et al., 2006). Twenty-seven isolates were taken from seeds collected from 2007 to 2010 in multiple locations across Kentucky. For inoculum preparation, dry corn was set to imbibe water for $16 \mathrm{~h}$ before autoclaving. After autoclaving, the corn was inoculated with PDA plugs of Fusarium graminearum, mixed with $0.2 \mathrm{~g}$ streptomycin in $50 \mathrm{ml}$ sterile water, covered and incubated at room temperature for 3 weeks until it was fully colonized by the fungus. At this point, the corn was manually spread on a sterilized plastic sheet until dry, put in mesh bags and stored in the freezer until used. On 14 April the corn was spread between rows at a rate of $11.86 \mathrm{gm}^{-2}$. Liquid nitrogen fertilizer ( $28 \%$ UAN) was applied in the spring at a rate of $105 \mathrm{~kg} \mathrm{~N} / \mathrm{ha}$ in split applications. Harmony Extra herbicide was applied on 20 April and a second application of Fusarium graminearuminfected corn was applied on 21 April.

\section{Phenotyping}

\section{Traditional methods}

Heading date was recorded when $50 \%$ of the spikes in the row had emerged. Plant height was measured at the soft dough stage. FHB traits measured included incidence, severity, FHB index (severity * incidence), visual rating (0-9, where 0 equals absence of 
FHB symptoms and 9 equals and FHB index $\geq 90 \%$ ), Fusarium damaged kernels (FDK) and deoxynivalenol (DON) concentration (ppm). ). Incidence was estimated by counting the number of blighted spikes in a random sample of 20 spikes in each row 24 days after heading. Severity was measured as the proportion of infected spikelets/total spikelets per spike, in 10 heads per row 24 days after heading. Samples were mechanically threshed and cleaned with low air flow to ensure minimal loss of scabby kernels. Approximately 45-g grain samples from each row were cleaned by hand and subsequently evaluated for FDK using an air separation machine specifically developed from a Precision Machine head thresher and a Shop-Vac vacuum to separate scabby kernels from healthy ones as described in (Agostinelli et al., 2012). FDK was expressed as the weight of scabby kernels divided by total weight. A 20-g sample in which scabby kernels had been recombined with sound kernels was analyzed for DON at University of Minnesota DON testing laboratory using gas chromatography with mass spectrometry (GC-MS) (Mirocha et al., 1998).

\section{Near infrared reflectance}

Samples were run on a NIR analyzer (DA7200, 950-1650 nm wavelength range) manufactured by Perten Instruments (IL). The calibration built in 2007 by the University of Kentucky Wheat Breeding Program and the manufacturers was used to run 2010 samples and it was updated on the basis of 2010 FDK and DON data measured by air separation and GC-MS, respectively, to run 2011 samples. The amount of seed required is approximately $20 \mathrm{~g}$.

\section{Data analysis}

Broad sense heritability of FHB and agronomic traits was estimated on an entry mean basis using the following model:

$\mathrm{Y}_{\mathrm{ij}}=\mu+\mathrm{G}_{\mathrm{k}}+\mathrm{R}(\mathrm{ENV})_{\mathrm{ij}}+\mathrm{ENV}_{\mathrm{i}} * \mathrm{G}_{\mathrm{j}}+\mathrm{E}_{\mathrm{ij}}$

Where: $\mathrm{Y}_{\mathrm{ij}}=$ the observation in the $k$ th genotype in the $j$ th rep in the $i$ th environment, $\mu=$ the overall mean, $\mathrm{G}_{\mathrm{j}}=$ the effect of the $k$ th genotype, $\mathrm{R}(\mathrm{ENV})_{\mathrm{ij}}=$ the effect of $j$ th rep within $i$ th environment, $\mathrm{ENV}_{\mathrm{i}} * \mathrm{G}_{\mathrm{j}}=$ the effect of the interaction of the $i$ th environment with the $k$ th genotype, $\mathrm{E}_{\mathrm{ij}}=$ the residual error. 
Data was analyzed using the General Linear Models procedure (Proc GLM; SAS 2002). Genotypic and phenotypic variances were estimated from the expected mean squares (EMS) and heritability estimates were computed as:

$\mathrm{h}^{2}=\mathrm{V}_{\mathrm{g}} / \mathrm{V}_{\mathrm{p}}$

where $\mathrm{h}^{2}=$ heritability, $\mathrm{V}_{\mathrm{g}}=$ genotypic variance, $\mathrm{V}_{\mathrm{p}}=$ phenotypic variance.

Confidence intervals (90\%) were calculated after Knapp et al. (1985) as:

$\mathrm{UL}=1-\left[\mathrm{MS}_{3} / \mathrm{MS}_{2} * \mathrm{~F}_{\mathrm{UL}}(.10, \mathrm{v} 1\right.$ and $\left.\mathrm{v} 2 \mathrm{df})\right]-1$

$\mathrm{LL}=1-\left[\mathrm{MS}_{3} / \mathrm{MS}_{2} * \mathrm{~F}_{\mathrm{LL}}(.90, \mathrm{v} 1\right.$ and $\left.\mathrm{v} 2 \mathrm{df})\right]-1$

Where: $\mathrm{UL}=$ upper limit of the confidence interval, $\mathrm{MS}_{3}=$ entry mean square, $\mathrm{MS}_{2}=$ residual mean square, $\mathrm{F}_{\mathrm{UL}}$ and $\mathrm{F}_{\mathrm{LL}}=\mathrm{F}$ value for the upper and lower limits calculated using the FINV function of Microsoft Excel (2007).

Proc CORR (SAS 2002) was used to analyze the relationship among traits on an entry mean basis. Entry means were plotted using Microsoft Excel (2007) to study the relationship among traits and calculate $\mathrm{R}^{2}$.

\section{Results and discussion}

\section{Heritability of FDK and DON measured using NIR}

Near infrared based FDK (NIRFDK) and DON (NIRDON) heritability estimates were 0.65 and 0.70 , respectively, for all populations combined (Table 5.1). Confidence intervals were relatively narrow $(0.7-0.6$ for NIRFDK, and $0.8-0.6$ for NIRDON). However, when each parameter was estimated by population, some differences were observed. Population 1 presented the highest heritability estimates $(0.77$ and 0.81 for NIRFDK and NIRDON, respectively). This highest NIRFDK $\mathrm{h}^{2}$ was followed by populations $3,4,2$ and 5 with estimates of $0.73,0.68,0.63$ and 0.59 , respectively. Accuracy of these estimates varied as well. For example, the lower limit of the confidence interval for NIRFDK $h^{2}$ in population 1 was 0 . NIRDON $h^{2}$ also varied among populations. The highest and lowest estimates were 0.81 and 0.55 , in populations 1 and 5 , respectively. 


\section{Relationship between FHB traits}

Table 5.2 presents Pearson correlation coefficients between NIR measurements and FHB traits measured with traditional methods. NIRDON-DON correlation was higher in $2011(\mathrm{r}=0.63)$ than in $2010(\mathrm{r}=0.56)$ and this may be due to having a more robust calibration for 2011 that was updated with 2010 data. FDKNIR measurements were moderately correlated with other traits such as rating $(r=0.50$ in 2011 , and $r=0.43$ in 2010), severity $(r=0.48$ in 2011 , and $r=0.51$ in 2010) and index $(r=0.41$ in 2011, and $r=0.52$ in 2010). The negative correlation found between FHB traits and height was also detected by NIR.

Whole grain NIRFDK explained $69 \%$ of the variation in FDK but this $\mathrm{R}^{2}$ varied among populations (Figure 5.1). The coefficient of determination of DON on NIRDON was 0.36 and it also varied among populations (Figure 5.2). Figures A.5.1 to A.5.5 in the appendix show the regression of FDK on NIRFDK for each population separately. $\mathrm{R}^{2}$ were $0.79,0.67,0.60,0.72$ and 0.60 for populations $1,2,3,4$ and 5 , respectively. NIRDON and DON coefficients of determination were $0.56,0.23,0.57,0.61$ and 0.32 for populations 1, 2, 3, 4 and 5, respectively (Figures A.5.6 to A.5.10 in the appendix)

Correlation between DON and NIRFDK (Figure 5.3) was higher than between DON and FDK measured by air separation (Figure 5.4). The coefficient of determination increased from 0.36 to 0.45 indicating that the NIR may be more accurately distinguishing scabby from healthy kernels. FDK ranged from 5 to $47 \%$ with a mean of $17 \%$, and from 7 to $32 \%$ with a mean of $19 \%$, when measured by air separation and with NIR, respectively (data not shown). When DON-NIRFDK relationship was analyzed by population, $\mathrm{R}^{2}$ decreased from 0.62 to 0.54 in population 1 (Figures A.5.11 and A.5.12), improved from 0.24 to 0.31 and 0.31 to 0.59 in populations 2 (Figures A.5.13 and A.5.14) and 3 (Figures A.5.15 and A.5.16), respectively, and maintained in pop 4 (Figures A.5.17 and A.5.18) and 5 (Figures A.5.19 and A.5.20). The fact that the NIR instrument provides a more complete assessment of FDK based on shape, color and composition could explain its better predictive ability. In contrast, FDK measured by air separation is based on only one aspect of the scabby kernels: the fact that these are lighter. As it was discussed before, there are other factors that can cause grains to be 
small, light and shriveled; so a more complete assessment that takes color and composition into account is likely to be more accurate at measuring FDK.

\section{Conclusions}

Moderate to strong correlations were found between FDK and DON measured using traditional methods and NIR which provides evidence for the potential use of this tool in breeding programs. The fact that DON was either better or equally correlated with NIRFDK than with FDK measured by air separation in four of five populations and overall, suggests that the NIR method could offer a better way to assess this trait. In addition, NIR has the advantage that it also provides a DON prediction. This could help accelerate the process of selecting for low DON because the number of samples sent to the lab could be reduced by discarding those with very high NIRFDK or NIRDON levels. 
Table 5.1: Heritability and 90\% confidence interval estimates in parentheses for NIRFDK and NIRDON based on a 2 year ANOVA of five wheat populations evaluated in Lexington 2010 and Princeton 2011.

\begin{tabular}{ccccc}
\hline & \multicolumn{2}{c}{$\begin{array}{c}\text { NIRFDK } \\
(\%)\end{array}$} & \multicolumn{2}{c}{$\begin{array}{c}\text { NIRDON } \\
(\mathrm{ppm})\end{array}$} \\
\hline Overall & 0.65 & $(0.7-0.6)$ & 0.70 & $(0.8-0.6)$ \\
Population 1 & 0.77 & $(0.9-0.6)$ & 0.81 & $(0.9-0.7)$ \\
Population 2 & 0.63 & $(0.7-0.0)$ & 0.75 & $(0.9-0.6)$ \\
Population 3 & 0.73 & $(0.8-0.6)$ & 0.73 & $(0.8-0.6)$ \\
Population 4 & 0.68 & $(0.8-0.5)$ & 0.62 & $(0.7-0.4)$ \\
Population 5 & 0.59 & $(0.8-0.3)$ & 0.55 & $(0.7-0.3)$ \\
\hline
\end{tabular}


Table 5.2: Genotype mean Pearson correlation coefficients for FHB traits and NIR measurements (NIRFDK, NIRDON) for all five populations in Lexington 2010 and 2011.

\begin{tabular}{lccccccccc}
\hline & \multicolumn{3}{c}{ NIRFDK } & \multicolumn{4}{c}{ NIRDON } \\
& \multicolumn{2}{c}{$\mathbf{2 0 1 1}$} & \multicolumn{2}{c}{$\mathbf{2 0 1 0}$} & \multicolumn{2}{c}{$\mathbf{2 0 1 1}$} & \multicolumn{2}{c}{$\mathbf{2 0 1 0}$} \\
\hline FDK & 0.73 & $<.0001$ & 0.70 & $<.0001$ & 0.71 & $<.0001$ & 0.51 & $<.0001$ \\
DON & 0.66 & $<.0001$ & 0.59 & $<.0001$ & 0.63 & $<.0001$ & 0.56 & $<.0001$ \\
Rating & 0.50 & $<.0001$ & 0.43 & $<.0001$ & 0.49 & $<.0001$ & 0.49 & $<.0001$ \\
Severity & 0.48 & $<.0001$ & 0.51 & $<.0001$ & 0.46 & $<.0001$ & 0.38 & $<.0001$ \\
Incidence & 0.25 & 0.0003 & 0.42 & $<.0001$ & 0.24 & 0.0005 & 0.36 & $<.0001$ \\
Index & 0.41 & $<.0001$ & 0.52 & $<.0001$ & 0.38 & $<.0001$ & 0.39 & $<.0001$ \\
Heading Date & & & 0.20 & 0.0089 & -0.15 & 0.0384 & & \\
Height & -0.34 & $<.0001$ & & & -0.44 & $<.0001$ & -0.35 & $<.0001$ \\
\hline
\end{tabular}


Figure 5.1: Regression of percentage of Fusarium damaged kernels measured by air separation (FDK) on percentage of Fusarium damaged kernels measured with NIR (NIRFDK) for all populations in Lexington 2010 and 2011.

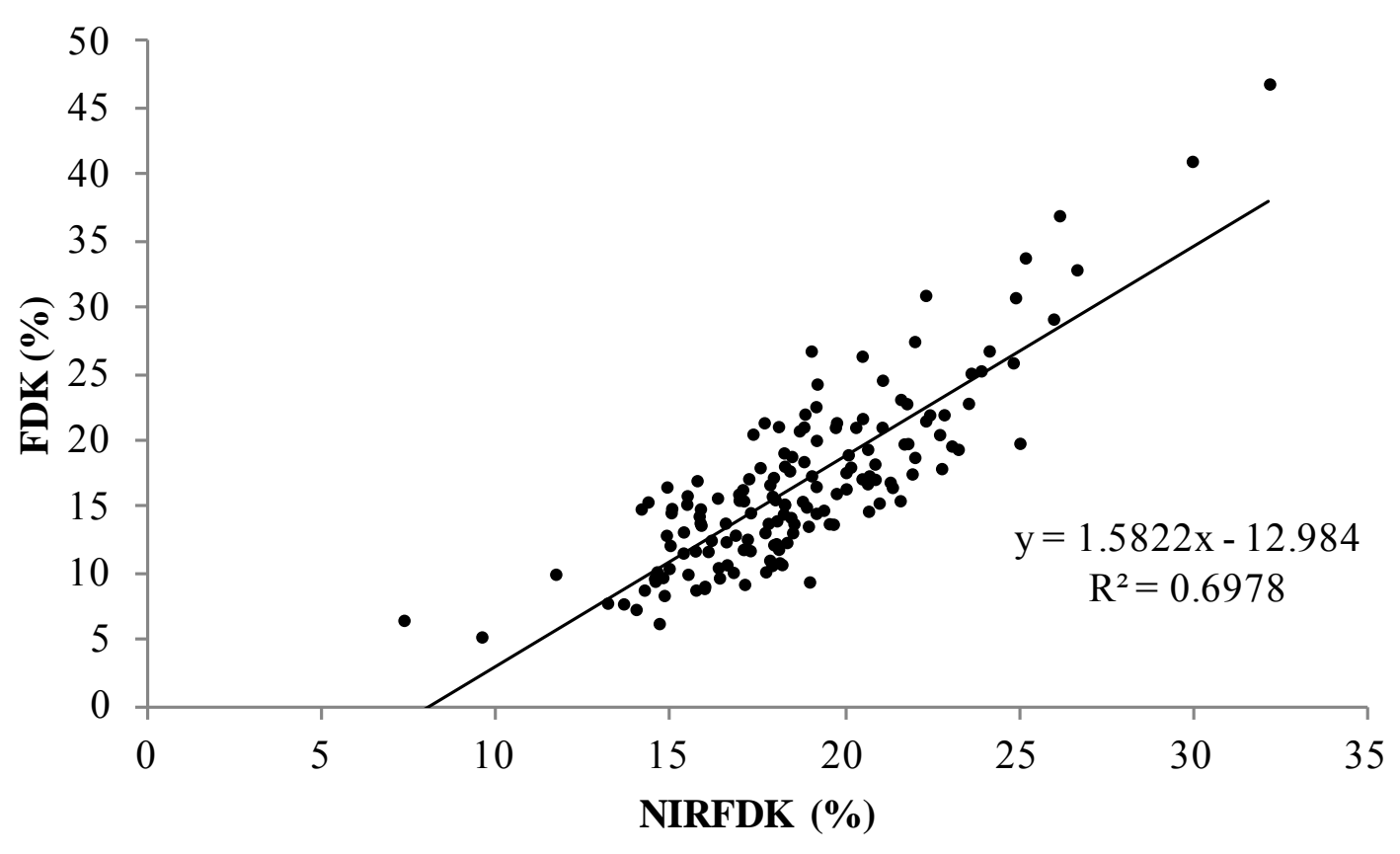


Figure 5.2: Regression of deoxynivalenol level measured by traditional methods (DON) on deoxynivalenol level measured with NIR (NIRDON) on for all populations in Lexington 2010 and 2011.

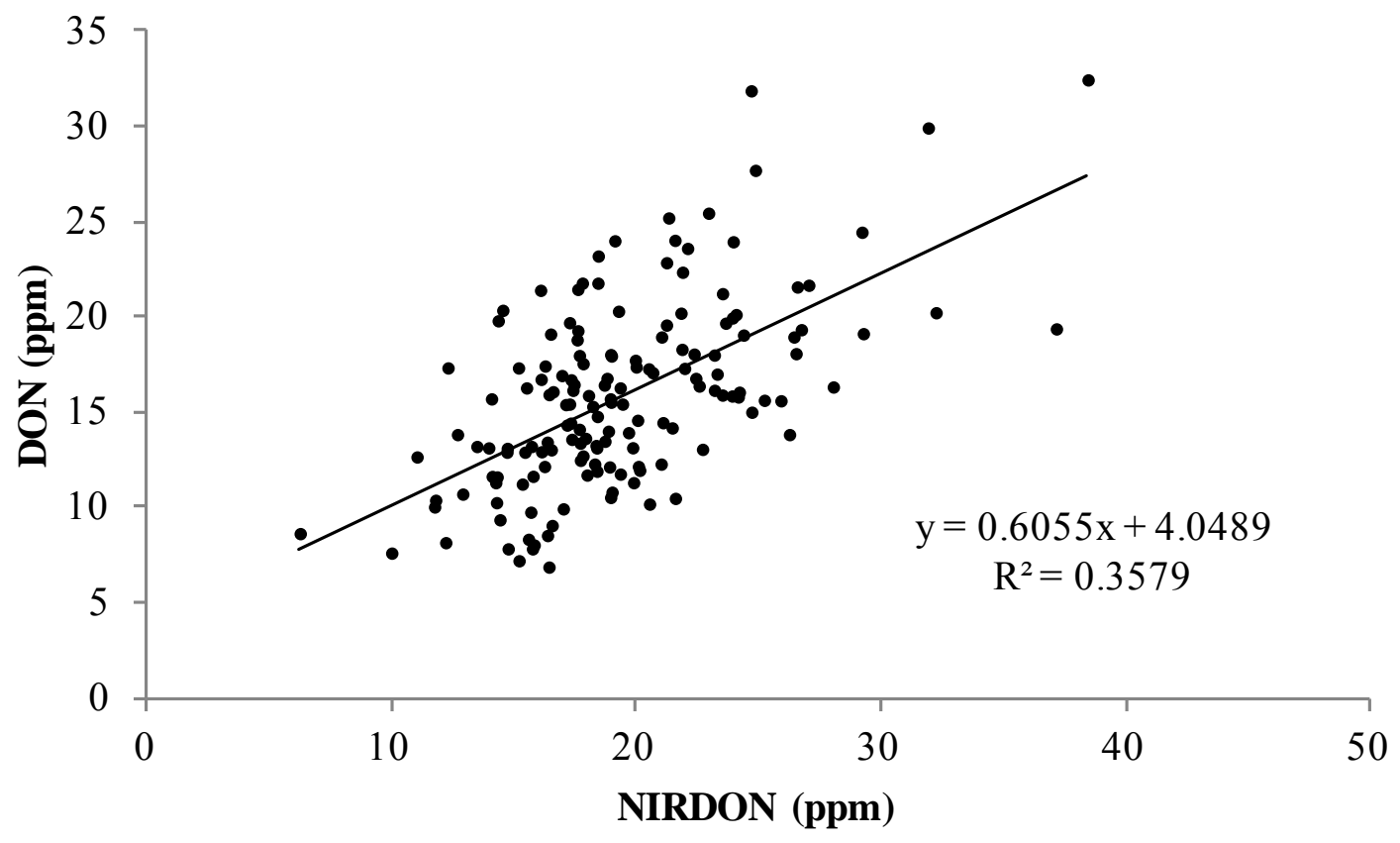


Figure 5.3: Regression of deoxynivalenol level (DON) on percentage of Fusarium damaged kernels measured with NIR (NIRFDK) for all populations in Lexington 2010 and 2011.

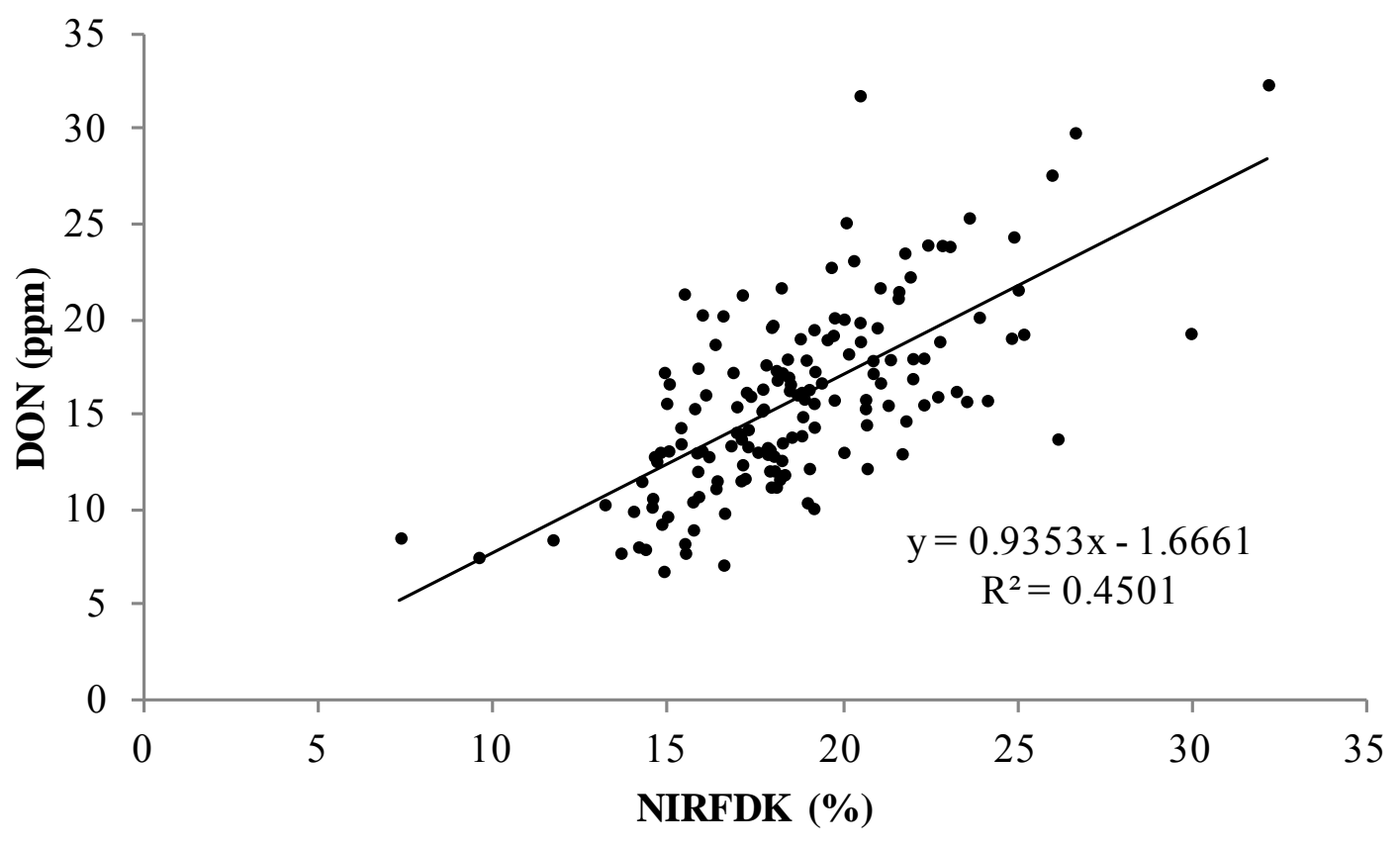


Figure 5.4: Regression of deoxynivalenol level (DON) on percentage of Fusarium damaged kernels (FDK) for all populations in Lexington 2010 and 2011.

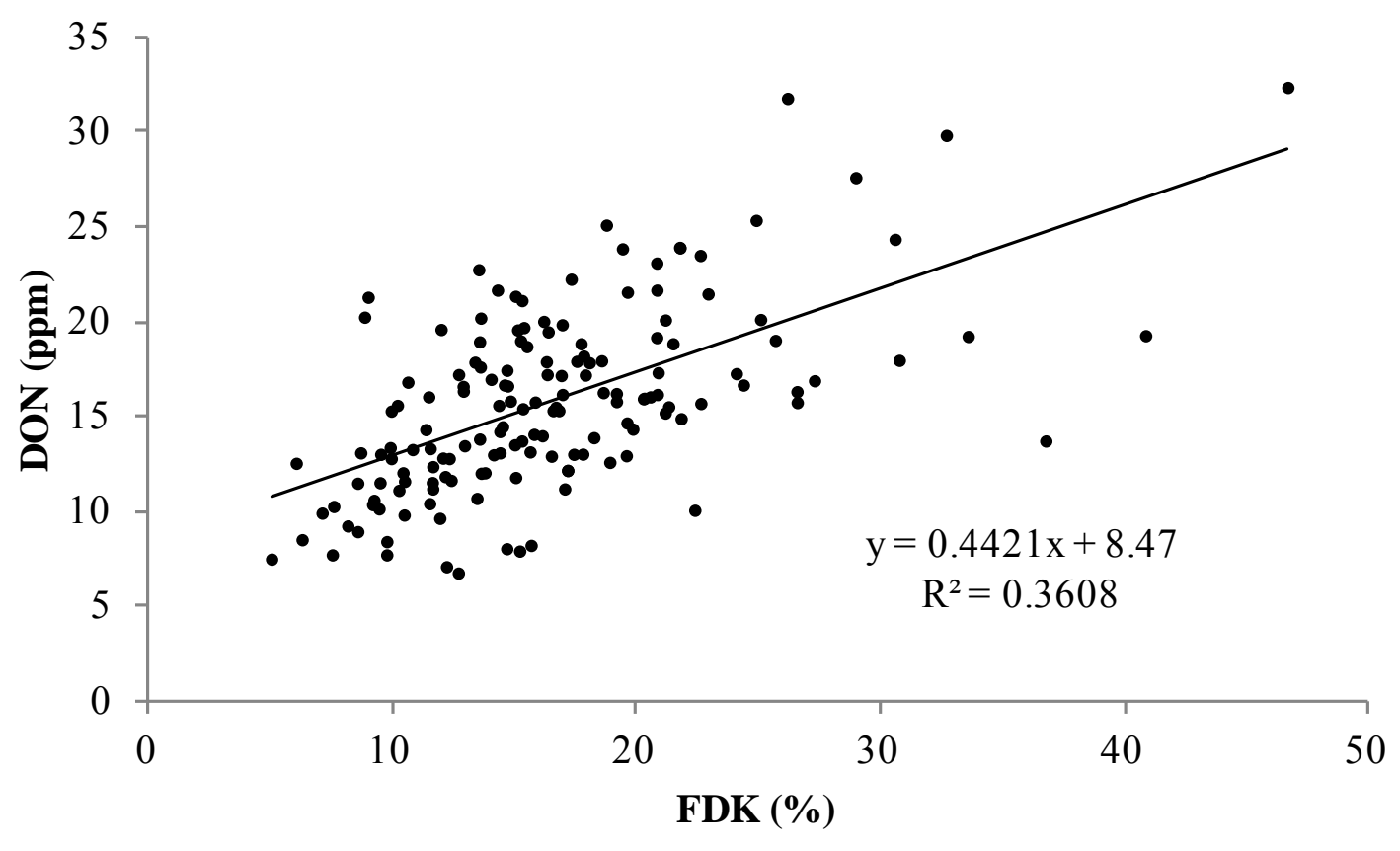




\section{APPENDIX}

\section{LIST OF TABLES}

Table A.3.1: Mean, Maximum (Max) and Minimum (Min) Fusarium damaged kernels (FDK), deoxynivalenol level (DON), FHB index, severity, incidence, FHB rating, heading date and plant height for five wheat populations and their different parents in the Lexington, KY scab nursery 2010 and 2011.

Table A.3.2: Mean, Maximum (Max) and Minimum (Min) Fusarium damaged kernels (FDK), deoxynivalenol level (DON), FHB index, severity, incidence, FHB rating, heading date and plant height for five wheat populations and their different parents in the Lexington, KY scab nursery 2010.

Table A.3.3: Mean, Maximum (Max) and Minimum (Min) Fusarium damaged kernels (FDK), deoxynivalenol level (DON), FHB index, severity, incidence, FHB rating, heading date and plant height for five wheat populations and their different parents in the Lexington, KY scab nursery 2011.

Table A.3.4: Mean Fusarium damaged kernels (FDK), deoxynivalenol level (DON), FHB index, severity, incidence, FHB rating, heading date and plant height for wheat lines homozygous for resistant (R) and susceptible (S) alleles at Fhb1, Lexington 2010 and 2011.

Table A.3.5: Mean Fusarium damaged kernels (FDK), deoxynivalenol level (DON), FHB index, severity, incidence, FHB rating, heading date and plant height for wheat lines homozygous for resistant (R) and susceptible (S) alleles at Fhb1, Lexington 2010.

Table A.3.6: Mean Fusarium damaged kernels (FDK), deoxynivalenol level (DON), FHB index, severity, incidence, FHB rating, heading date and plant height for wheat lines homozygous for resistant (R) and susceptible (S) alleles at Fhb1, Lexington 2011..... 98

Table A.3.7: Mean Fusarium damaged kernels (FDK), deoxynivalenol level (DON), FHB index, severity, incidence, FHB rating, heading date and plant height for homozygous susceptible $(\mathrm{S})$, resistant $(\mathrm{R})$ and heterozygous $(\mathrm{H})$ wheat lines at QFhs.nau$2 D L$, Lexington 2010 and 2011.

Table A.3.8: Mean Fusarium damaged kernels (FDK), deoxynivalenol level (DON), FHB index, severity, incidence, FHB rating, heading date and plant height for homozygous susceptible $(\mathrm{S})$, resistant $(\mathrm{R})$ and heterozygous $(\mathrm{H})$ wheat lines at QFhs.nau2DL, Lexington 2010. 
Table A.3.9: Mean Fusarium damaged kernels (FDK), deoxynivalenol level (DON), FHB index, severity, incidence, FHB rating, heading date and plant height for homozygous susceptible (S), resistant (R) and heterozygous (H) wheat lines at QFhs.nau2DL, Lexington 2011.

Table A.3.10: Mean Fusarium damaged kernels (FDK), deoxynivalenol level (DON), FHB index, severity, incidence, FHB rating, heading date and plant height for wheat lines in each QTL combination class, Lexington 2010 and 2011.

Table A.3.11: Mean Fusarium damaged kernels (FDK), deoxynivalenol level (DON), FHB index, severity, incidence, FHB rating, heading date and plant height for wheat lines in each QTL combination class when all populations were combined, Lexington 2010 and 2011. 104

Table A.3.12: Heritabilities and their 90\% confidence intervals in parentheses, based on 2 year ANOVA of five wheat populations. Traits evaluated were: deoxynivalenol level (DON), Fusarium damaged kernels (FDK), FHB rating, FHB index, severity and

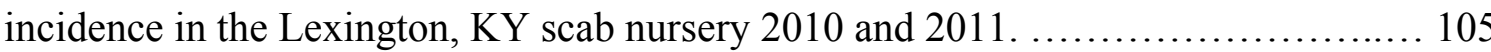

Table A.3.13: Genotype mean Pearson correlation coefficients between Fusarium damaged kernels (FDK), deoxynivalenol level (DON), FHB rating, severity, incidence, FHB index, heading date and plant height, in Lexington, KY scab nursery 2010 and 2011.

Table A.3.14: Mean, Maximum (Max) and Minimum (Min) yield, test weight (TWT), plant height and heading date (HD) for five populations and their different parents in Lexington 2010 and 2011, and Princeton 2011.

Table A.3.15: Mean, Maximum (Max) and Minimum (Min) yield, test weight (TWT), plant height and heading date (HD) for five populations and their different parents in Lexington 2010.................................................................. 108

Table A.3.16: Mean, Maximum (Max) and Minimum (Min) yield, test weight (TWT), plant height and heading date (HD) for five populations and their different parents in

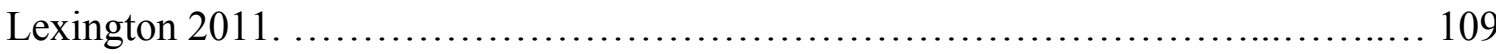

Table A.3.17: Mean, Maximum (Max) and Minimum (Min) yield, test weight (TWT), plant height and heading date (HD) for five populations and their different parents in

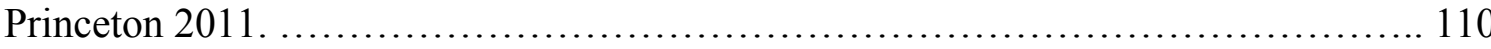

Table A.3.18: Mean yield, test weight (TWT), plant height and heading date (HD) for wheat lines homozygous for resistant (R) and susceptible (S) alleles at Fhb1, Lexington 2010 and 2011, and Princeton 2011. 
Table A.3.19: Mean yield, test weight (TWT), plant height and heading date (HD) for wheat lines homozygous for resistant (R) and susceptible (S) alleles at Fhb1, Lexington 2010 .

Table A.3.20: Mean yield, test weight (TWT), plant height, heading date (HD) and FHB rating measured in plots for wheat lines homozygous for resistant $(\mathrm{R})$ and susceptible $(\mathrm{S})$ alleles at $F h b 1$, Lexington 2011.

Table A.3.21: Mean yield, test weight (TWT), plant height, heading date (HD) and FHB rating measured in plots for wheat lines homozygous for resistant $(\mathrm{R})$ and susceptible $(\mathrm{S})$ alleles at $F h b 1$, Princeton 2011.

Table A.3.22: Mean yield, test weight (TWT), plant height and heading date (HD) for homozygous susceptible $(\mathrm{S})$, resistant $(\mathrm{R})$ and heterozygous $(\mathrm{H})$ wheat lines at QFhs.nau2DL, Lexington 2010 and 2011, and Princeton 2011.

Table A.3.23: Mean yield, test weight (TWT), plant height and heading date (HD) for homozygous susceptible $(\mathrm{S})$, resistant $(\mathrm{R})$ and heterozygous $(\mathrm{H})$ wheat lines at QFhs.nau2DL, Lexington 2010.

Table A.3.24: Mean yield, test weight (TWT), plant height, heading date (HD) and FHB rating measured in plots for homozygous susceptible (S), resistant (R) and heterozygous

(H) wheat lines at QFhs.nau-2DL, Lexington 2011.

Table A.3.25: Mean yield, test weight (TWT), plant height, heading date (HD) and FHB rating measured in plots for homozygous susceptible (S), resistant (R) and heterozygous (H) wheat lines at QFhs.nau-2DL, Princeton 2011.

Table A.3.26: Mean yield, test weight (TWT), plant height and heading date (HD) for wheat lines in each QTL combination class when all populations were combined, Lexington 2010 and 2011, and Princeton 2011.

Table A.3.27: Mean, maximum (Max) and minimum (Min) whole grain hardness (WGH), whole grain protein (WGP), flour yield (FY), softness equivalent (SEQ) and flour protein (FP) for five wheat populations and their parents, Lexington 2010 and Princeton 2011.

Table A.3.28: Mean, maximum (Max) and minimum (Min) whole grain hardness (WGH), whole grain protein (WGP), flour yield (FY), softness equivalent (SEQ) and flour protein (FP) for five wheat populations and their parents, Lexington 2010.

Table A.3.29: Mean, maximum (Max) and minimum (Min) whole grain hardness (WGH), whole grain protein (WGP), flour yield (FY), softness equivalent (SEQ) and flour protein (FP) for five wheat populations and their parents, Princeton 2011. 
Table A.3.30: Mean, maximum (Max) and minimum (Min) Gluten performance index (GPI), flour lactic acid solvent retention capacity (LASRC), flour sucrose solvent retention capacity (SSRC), flour water solvent retention capacity (WSRC), flour sodium carbonate solvent retention capacity (SCSRC), estimated cookie diameter (ECD), wheat meal sedimentation test (SDS) and wheat meal SCSRC (WMSCSRC) for five wheat populations and their parents in Lexington 2010 and Princeton 2011.

Table A.3.31: Mean, maximum (Max) and minimum (Min) Gluten performance index (GPI), flour lactic acid solvent retention capacity (LASRC), flour sucrose solvent retention capacity (SSRC), flour water solvent retention capacity (WSRC), flour sodium carbonate solvent retention capacity (SCSRC), estimated cookie diameter (ECD), wheat meal sedimentation test (SDS) and wheat meal SCSRC (WMSCSRC) for five wheat populations and their parents in Lexington 2010 ............................. 128

Table A.3.32: Mean, maximum (Max) and minimum (Min) Gluten performance index (GPI), flour lactic acid solvent retention capacity (LASRC), flour sucrose solvent retention capacity (SSRC), flour water solvent retention capacity (WSRC), flour sodium carbonate solvent retention capacity (SCSRC), estimated cookie diameter (ECD), wheat meal sedimentation test (SDS) and wheat meal SCSRC (WMSCSRC) for five wheat populations and their parents in Princeton 2011 ................................. 130

Table A.3.33: Mean whole grain hardness (WGH), whole grain protein (WGP), flour yield (FY), softness equivalent (SEQ) and flour protein (FP) for wheat lines homozygous for resistant (R) and susceptible (S) alleles at Fhb1, Lexington 2010 and Princeton 2011.

Table A.3.34: Mean gluten performance index (GPI), flour lactic acid solvent retention capacity (LASRC), flour sucrose solvent retention capacity (SSRC), flour water solvent retention capacity (WSRC), flour sodium carbonate solvent retention capacity (SCSRC), wheat meal sedimentation test (SDS) and wheat meal SCSRC (WMSCSRC) for wheat lines homozygous for resistant (R) and susceptible (S) alleles at Fhb1, Lexington 2010 and Princeton 2011

Table A.3.35: Mean whole grain hardness (WGH), whole grain protein (WGP), flour yield (FY), softness equivalent (SEQ) and flour protein (FP) for wheat lines homozygous for resistant (R) and susceptible (S) alleles at Fhb1, Lexington 2010 ............... 134

Table A.3.36: Mean gluten performance index (GPI), flour lactic acid solvent retention capacity (LASRC), flour sucrose solvent retention capacity (SSRC), flour water solvent retention capacity (WSRC), flour sodium carbonate solvent retention capacity (SCSRC), estimated cookie diameter (ECD), wheat meal sedimentation test (SDS) and wheat meal SCSRC (WMSCSRC) for wheat lines homozygous for resistant (R) and susceptible (S)

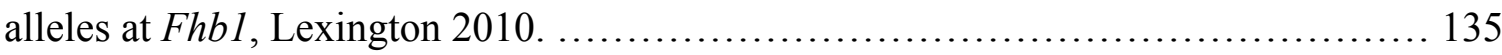


Table A.3.37: Mean whole grain hardness (WGH), whole grain protein (WGP), flour yield (FY), softness equivalent (SEQ) and flour protein (FP) for wheat lines homozygous for resistant (R) and susceptible (S) alleles at Fhb1, Princeton 2011 ................. 136

Table A.3.38: Mean gluten performance index (GPI), flour lactic acid solvent retention capacity (LASRC), flour sucrose solvent retention capacity (SSRC), flour water solvent retention capacity (WSRC), flour sodium carbonate solvent retention capacity (SCSRC), wheat meal sedimentation test (SDS) and wheat meal SCSRC (WMSCSRC) for wheat lines homozygous for resistant (R) and susceptible (S) alleles at Fhb1, Princeton 2011.

Table A.3.39: Mean whole grain hardness (WGH), whole grain protein (WGP), flour yield (FY), softness equivalent (SEQ) and flour protein (FP) for homozygous susceptible (S), resistant (R) and heterozygous (H) wheat lines at QFhs.nau-2DL, Lexington 2010 and Princeton 2011.............................................................. 138

Table A.3.40: Mean gluten performance index (GPI), flour lactic acid solvent retention capacity (LASRC),flour sucrose solvent retention capacity (SSRC), flour water solvent retention capacity (WSRC), sodium carbonate solvent retention capacity (SCSRC), wheat meal sedimentation test (SDS) and wheat meal SCSRC (WMSCSRC) for homozygous susceptible (S), resistant (R) and heterozygous (H) wheat lines at $Q F h$ s.nau-2DL, Lexington 2010 and Princeton 2011............................................. 140

Table A.3.41: Mean whole grain hardness (WGH), whole grain protein (WGP), flour yield (FY), softness equivalent (SEQ) and flour protein (FP) for homozygous susceptible (S), resistant (R) and heterozygous (H) wheat lines at QFhs.nau-2DL, Lexington 2010.

Table A.3.42: Mean gluten performance index (GPI), flour lactic acid solvent retention capacity (LASRC),flour sucrose solvent retention capacity (SSRC), flour water solvent retention capacity (WSRC), sodium carbonate solvent retention capacity (SCSRC), estimated cookie diameter, wheat meal sedimentation test (SDS) and wheat meal SCSRC (WMSCSRC) for homozygous susceptible (S), resistant (R) and heterozygous $(\mathrm{H})$ wheat

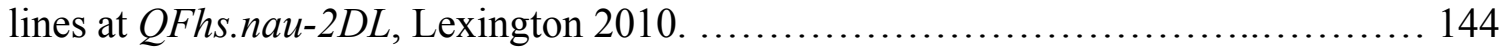

Table A.3.43: Mean whole grain hardness (WGH), whole grain protein (WGP), flour yield (FY), softness equivalent (SEQ) and flour protein (FP) for homozygous susceptible (S), resistant (R) and heterozygous (H) wheat lines at QFhs.nau-2DL, Princeton 2011.

Table A.3.44: Mean gluten performance index (GPI), flour lactic acid solvent retention capacity (LASRC), flour sucrose solvent retention capacity (SSRC), flour water solvent retention capacity (WSRC), sodium carbonate solvent retention capacity (SCSRC), wheat 
meal sedimentation test (SDS) and wheat meal SCSRC (WMSCSRC) for homozygous susceptible (S), resistant $(\mathrm{R})$ and heterozygous $(\mathrm{H})$ wheat lines at QFhs.nau-2DL,

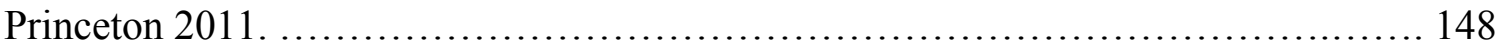

Table A.3.45: Mean whole grain hardness (WGH), whole grain protein (WGP), flour yield (FY), softness equivalent (SEQ) and flour protein (FP) for wheat lines in each QTL combination class when all populations were combined, Lexington 2010 and Princeton

2011 150

Table A.3.46: Mean gluten performance index (GPI), flour lactic acid solvent retention capacity (LASRC), flour sucrose solvent retention capacity (SSRC), flour water solvent retention capacity (WSRC), flour sodium carbonate solvent retention capacity (SCSRC), wheat meal sedimentation test (SDS) and wheat meal SCSRC (WMSCSRC) for wheat lines in each QTL combination class when all populations were combined, Lexington 2010 and Princeton 2011.

Table A.3.47: Heritabilities and their 90\% confidence intervals in parentheses based on 3 year ANOVA of five wheat populations. Traits evaluated were: yield, test weight (TWT), heading date (HD) and plant height, Lexington 2010 and 2011, and Princeton 2011.

Table A.3.48: Heritabilities and their 90\% confidence intervals in parentheses based on 2 year ANOVA of five wheat populations. Traits evaluated were: whole grain hardness (WGH), whole grain protein (WGP), flour yield (FY), softness equivalent (SEQ) and flour protein (FP), Lexington 2010 and Princeton 2011.

Table A.3.49: Heritabilities and their 90\% confidence intervals in parentheses based on 2 year ANOVA of five wheat populations. Traits evaluated were: gluten performance index (GPI), flour lactic acid solvent retention capacity (LASRC), flour sucrose solvent retention capacity (SSRC), flour water solvent retention capacity (WSRC) and flour sodium carbonate solvent retention capacity (SCSRC), Lexington 2010 and Princeton 2011.

Table A.3.50: Genotype mean Pearson correlation coefficients between test weight (TWT) whole grain hardness (WGH), whole grain protein (WGP), flour yield (FY), softness equivalent (SEQ) and flour protein (FP), gluten performance index (GPI), flour lactic acid solvent retention capacity (LASRC), flour sucrose solvent retention capacity (SSRC), flour water solvent retention capacity (WSRC) and flour sodium carbonate solvent retention capacity (SCSRC), Lexington 2010 and Princeton 2011.

Table A.3.51: Mean Fusarium damaged kernels (FDK), deoxynivalenol level (DON), FHB index, severity, incidence, FHB rating, heading date and plant height for wheat lines 
homozygous for resistant (R) and susceptible (S) alleles at Fhb1, Lexington 2011 (Increase lines included).

Table A.3.52: Mean yield, test weight (TWT), plant height, heading date (HD) and FHB rating measured in plots for wheat lines homozygous for resistant (R) and susceptible (S) alleles at $F h b 1$, Lexington 2011 (Increase lines included).

Table A.3.53: Mean yield, test weight (TWT), plant height, heading date (HD) and FHB rating measured in plots for wheat lines homozygous for resistant $(\mathrm{R})$ and susceptible (S) alleles at Fhbl, Princeton 2011 (Increase lines included).

Table A.3.54: Mean whole grain hardness (WGH), whole grain protein (WGP), flour yield (FY), softness equivalent (SEQ) and flour protein (FP) for wheat lines homozygous for resistant (R) and susceptible (S) alleles at Fhb1, Princeton 2011 (Increase lines included).

Table A.3.55: Mean gluten performance index (GPI), flour lactic acid solvent retention capacity (LASRC), flour sucrose solvent retention capacity (SSRC), flour water solvent retention capacity (WSRC), flour sodium carbonate solvent retention capacity (SCSRC), wheat meal sedimentation test (SDS) and wheat meal SCSRC (WMSCSRC) for wheat lines homozygous for resistant (R) and susceptible (S) alleles at Fhb1, Princeton 2011 (Increase lines included).

Table A.3.56: Mean Fusarium damaged kernels (FDK), deoxynivalenol level (DON), FHB index, severity, incidence, FHB rating, heading date and plant height for wheat lines homozygous susceptible (S), resistant $(\mathrm{R})$ and heterozygous $(\mathrm{H})$ wheat lines at QFhs.nau$2 D L$, Lexington 2011 (Increase lines included).

Table A.3.57: Mean yield, test weight (TWT), plant height, heading date (HD) and FHB rating measured in plots for homozygous susceptible (S), resistant (R) and heterozygous (H) wheat lines at QFhs.nau-2DL, Lexington 2011 (Increase lines included). 162

Table A.3.58: Mean yield, test weight (TWT), plant height, heading date (HD) and FHB rating measured in plots for homozygous susceptible (S), resistant $(\mathrm{R})$ and heterozygous (H) wheat lines at $Q F h s . n a u-2 D L$, Princeton 2011 (Increase lines included). 163

Table A.3.59: Mean whole grain hardness (WGH), whole grain protein (WGP), flour yield (FY), softness equivalent (SEQ) and flour protein (FP) for homozygous susceptible (S), resistant (R) and heterozygous (H) wheat lines at QFhs.nau-2DL, Princeton 2011 (Increase lines included). 164

Table A.3.60: Mean gluten performance index (GPI), flour lactic acid solvent retention capacity (LASRC),flour sucrose solvent retention capacity (SSRC), flour water solvent retention capacity (WSRC), sodium carbonate solvent retention capacity (SCSRC), wheat 
meal sedimentation test (SDS) and wheat meal SCSRC (WMSCSRC) for homozygous susceptible (S), resistant (R) and heterozygous (H) wheat lines at $Q F h s . n a u-2 D L$, Princeton 2011 (Increase lines included). 


\section{LIST OF FIGURES}

Figure A.3.1: Precipitation and mean temperature during wheat growth season in Lexington 2010. Black bars and empty bars correspond 2010 and historic precipitation levels, respectively. 166

Figure A.3.2: Precipitation and mean temperature during wheat growth season in Lexington 2011. Black bars and empty bars correspond 2011 and historic precipitation levels, respectively.

Figure A.3.3: Precipitation and mean temperature during wheat growth season in Princeton 2011. Black bars and empty bars correspond 2011 and historic precipitation levels, respectively.

Figure A.3.4: Regression of deoxynivalenol level (DON) on percentage of Fusarium damaged kernels (FDK) for all populations in Lexington 2010 and 2011 ............. 169

Figure A.3.5: Regression of deoxynivalenol level (DON) on percentage of Fusarium damaged kernels (FDK) in population 1 in Lexington 2010 and 2011 .............. 170

Figure A.3.6: Regression of deoxynivalenol level (DON) on percentage of Fusarium damaged kernels (FDK) in population 2 in Lexington 2010 and 2011.

Figure A.3.7: Regression of deoxynivalenol level (DON) on percentage of Fusarium damaged kernels (FDK) in population 3 in Lexington 2010 and 2011 .............. 172

Figure A.3.8: Regression of deoxynivalenol level (DON) on percentage of Fusarium damaged kernels (FDK) in population 4 in Lexington 2010 and 2011 ............... 173

Figure A.3.9: Regression of deoyinivalenol level (DON) on percentage of Fusarium damaged kernels (FDK) in population 5 in Lexington 2010 and 2011 ............... 174

Figure A.3.10: Regression of deoxynivalenol level (DON) on Fusarium damaged kernels (FDK) for all populations in 2010 and 2011. 175

Figure A.3.11: Regression of deoxynivalenol level (DON) on Fusarium damaged kernels (FDK) for populations 1 in Lexington 2010 and 2011. 176

Figure A.3.12: Regression of deoxynivalenol level (DON) on Fusarium damaged kernels (FDK) for populations 2 in Lexington 2010 and 2011.

Figure A.3.13: Regression of deoxynivalenol level (DON) on Fusarium damaged kernels (FDK) for populations 3 in Lexington 2010 and 2011. 178

Figure A.3.14: Regression of deoxynivalenol level (DON) on Fusarium damaged kernels (FDK) for populations 4 in Lexington 2010 and 2011. 179 
Figure A.3.15: Regression of deoxynivalenol level (DON) on Fusarium damaged kernels (FDK) for populations 5 in Lexington 2010 and 2011.

Figure A.3.16: Regression of Fusarium damaged kernels (FDK) on FHB index for all populations in Lexington 2010 and 2011.

Figure A.3.17: Regression of Fusarium damaged kernels (FDK) on FHB rating for all populations in Lexington 2010 and 2011. 182

Figure A.3.18: Regression of FHB index on FHB rating for all populations in Lexington 2010 and 2011. 183

Fig A.3.19: Regression of Fusarium damaged kernels (FDK) on FHB index for all populations in Lexington 2010 and 2011.

Figure A.3.20: Regression of Fusarium damaged kernels (FDK) on FHB rating for all populations in Lexington 2010 and 2011. 185

Figure A.3.21: Regression of FHB index on FHB rating for all populations in Lexington 2010 and 2011. 186

Figure A.4.1: Regression of flour yield on wheat meal (WM) sodium carbonate solvent retention capacity (SRC) in population 1 in Lexington 2010 and Princeton 2011.

Figure A.4.2: Regression of flour yield on wheat meal (WM) sodium carbonate solvent retention capacity (SRC) in population 2 in Lexington 2010 and Princeton 2011.

Figure A.4.3: Regression of flour yield on wheat meal (WM) sodium carbonate solvent retention capacity (SRC) in population 3 in Lexington 2010 and Princeton 2011.

Figure A.4.4: Regression of flour yield on wheat meal (WM) sodium carbonate solvent retention capacity (SRC) in population 4 in Lexington 2010 and Princeton 2011.

Figure A.4.5: Regression of flour yield on wheat meal (WM) sodium carbonate solvent retention capacity (SRC) in population 5 in Lexington 2010 and Princeton 2011.

Figure A.4.6: Regression of flour sucrose solvent retention capacity (SRC) on wheat meal (WM) sodium carbonate solvent retention capacity (SRC) in population 1 in Lexington 2010 and Princeton 2011. 
Figure A.4.7: Regression of flour sucrose solvent retention capacity (SRC) on wheat meal (WM) sodium carbonate solvent retention capacity (SRC) in population 2 in Lexington 2010 and Princeton 2011. 193

Figure A.4.8: Regression of flour sucrose solvent retention capacity (SRC) on wheat meal (WM) sodium carbonate solvent retention capacity (SRC) in population 3 in Lexington 2010 and Princeton 2011. 194

Figure A.4.9: Regression of flour sucrose solvent retention capacity (SRC) on wheat meal (WM) sodium carbonate solvent retention capacity (SRC) in population 4 in Lexington 2010 and Princeton 2011. 195

Figure A.4.10: Regression of flour sucrose solvent retention capacity (SRC) on wheat meal (WM) sodium carbonate solvent retention capacity (SRC) in population 5 in Lexington 2010 and Princeton 2011.

Figure A.4.11: Regression of flour lactic acid solvent retention capacity (SRC) on wheat meal (WM) SDS sedimentation in population 1 in Lexington 2010 and Princeton 2011.

Figure A.4.12: Regression of flour lactic acid solvent retention capacity (SRC) on wheat meal (WM) SDS sedimentation in population 2 in Lexington 2010 and Princeton 2011.

Figure A.4.13: Regression of flour lactic acid solvent retention capacity (SRC) on wheat meal (WM) SDS sedimentation in population 3 in Lexington 2010 and Princeton 2011.

Figure A.4.14: Regression of flour lactic acid solvent retention capacity (SRC) on wheat meal (WM) SDS sedimentation in population 4 in Lexington 2010 and Princeton 2011.

Figure A.4.15: Regression of flour lactic acid solvent retention capacity (SRC) on wheat meal (WM) SDS sedimentation in population 5 in Lexington 2010 and Princeton 2011.

Figure A.4.16: Regression of softness equivalent on NIR softness equivalent in population 1 in Lexington 2010 and Princeton 2011 ............................ 202

Figure A.4.17: Regression of softness equivalent on NIR softness equivalent in population 2 in Lexington 2010 and Princeton 2011 ........................... 203

Figure A.4.18: Regression of softness equivalent on NIR softness equivalent in population 3 in Lexington 2010 and Princeton 2011. 
Figure A.4.19: Regression of softness equivalent on NIR softness equivalent in population 4 in Lexington 2010 and Princeton 2011. 205

Figure A.4.20: Regression of softness equivalent on NIR softness equivalent in population 5 in Lexington 2010 and Princeton 2011. 206

Figure A.4.21: Regression of flour water solvent retention capacity (SRC) on NIR water SRC in population 1 in Lexington 2010 and Princeton 2011.

Figure A.4.22: Regression of flour water solvent retention capacity (SRC) on NIR water SRC in population 2 in Lexington 2010 and Princeton 2011. 208

Figure A.4.23: Regression of flour water solvent retention capacity (SRC) on NIR water SRC in population 3 in Lexington 2010 and Princeton 2011. 209

Figure A.4.24: Regression of flour water solvent retention capacity (SRC) on NIR water SRC in population 4 in Lexington 2010 and Princeton 2011. 210

Figure A.4.25: Regression of flour water solvent retention capacity (SRC) on NIR water SRC in population 5 in Lexington 2010 and Princeton 2011. 211

Figure A.5.1: Regression of percentage of Fusarium damaged kernels measured by air separation (FDK) on percentage of Fusarium damaged kernels measured with NIR (NIRFDK) in population 1.

Figure A.5.2: Regression of percentage of Fusarium damaged kernels measured by air separation (FDK) on percentage of Fusarium damaged kernels measured with NIR (NIRFDK) in population 2.

Figure A.5.3: Regression of percentage of Fusarium damaged kernels measured by air separation (FDK) on percentage of Fusarium damaged kernels measured with NIR (NIRFDK) in population 3 ................................................. 214

Figure A.5.4: Regression of percentage of Fusarium damaged kernels measured by air separation (FDK) on percentage of Fusarium damaged kernels measured with NIR

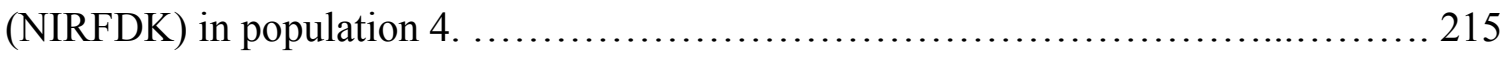

Figure A.5.5: Regression of percentage of Fusarium damaged kernels measured by air separation (FDK) on percentage of Fusarium damaged kernels measured with NIR (NIRFDK) in population 5................................................... 216

Figure A.5.6: Regression of deoxynivalenol level measured by traditional methods (DON) on deoxynivalenol level measured with NIR (NIRDON) in population 1. 
Figure A.5.7: Regression of deoxynivalenol level measured by traditional methods (DON) on deoxynivalenol level measured with NIR (NIRDON) in population 2.

Figure A.5.8: Regression of deoxynivalenol level measured by traditional methods (DON) on deoxynivalenol level measured with NIR (NIRDON) in population 3.

Figure A.5.9: Regression of deoxynivalenol level measured by traditional methods (DON) on deoxynivalenol level measured with NIR (NIRDON) in population 4.

Figure A.5.10: Regression of deoxynivalenol level measured by traditional methods (DON) on deoxynivalenol level measured with NIR (NIRDON) in population 5.

Figure A.5.11: Regression of deoxynivalenol level measured by traditional methods (DON) on percentage of fusarium damaged kernels measured with NIR (NIRFDK) in population 1 .

Figure A.5.12: Regression of deoxynivalenol level measured by traditional methods (DON) on percentage of Fusarium damaged kernels measured by air separation (FDK) in population 1 .

Figure A.5.13: Regression of deoxynivalenol level measured by traditional methods (DON) on percentage of Fusarium damaged kernels measured with NIR (NIRFDK) in

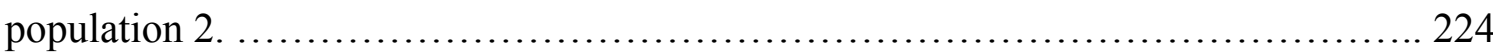

Figure A.5.14: Regression of deoxynivalenol level measured by traditional methods (DON) on percentage of Fusarium damaged kernels measured by air separation (FDK) in population 2

Figure A.5.15: Regression of deoxynivalenol level measured by traditional methods (DON) on percentage of Fusarium damaged kernels measured with NIR (NIRFDK) in population 3 .

Figure A.5.16: Regression of deoxynivalenol level measured with traditional methods (DON) on percentage of Fusarium damaged kernels measured by air separation (FDK) in population 3 .

Figure A.5.17: Regression of deoxynivalenol level measured with traditional methods (DON) on percentage of Fusarium damaged kernels measured with NIR (NIRFDK) in

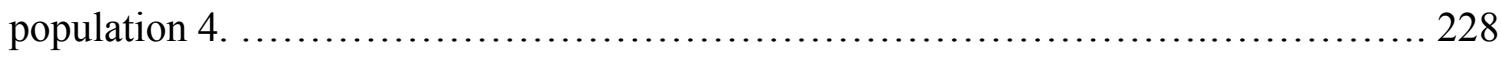


Figure A.5.18: Regression of deoxynivalenol level measured with traditional methods (DON) on percentage of Fusarium damaged kernels measured by air separation (FDK) in

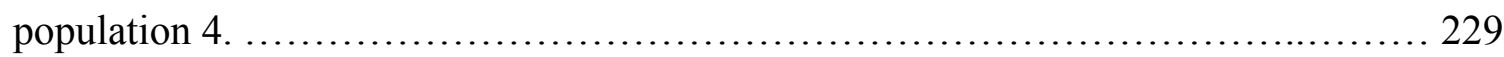

Figure A.5.19: Regression of deoxynivalenol level measured with traditional methods (DON) on percentage of Fusarium damaged kernels measured with NIR (NIRFDK) in

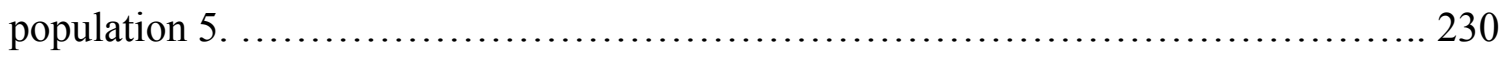

Figure A.5.20: Regression of deoxinivalenol level measured with traditional methods (DON) on percentage of Fusarium damaged kernels measured by air separation (FDK) in

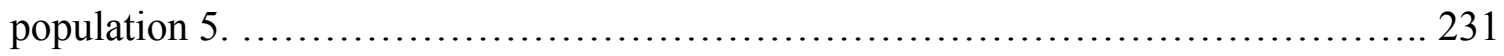


Table A.3.1: Mean, Maximum (Max) and Minimum (Min) Fusarium damaged kernels (FDK), deoxynivalenol level (DON), FHB index, severity, incidence, FHB rating, heading date and plant height for five wheat populations and their different parents in the Lexington, KY scab nursery 2010 and 2011.

\begin{tabular}{|c|c|c|c|c|c|c|c|c|c|}
\hline $\mathbf{N}$ & v & $\begin{array}{l}\text { FDK } \\
(\%)\end{array}$ & $\begin{array}{l}\text { DON } \\
\text { (ppm) }\end{array}$ & Index & $\begin{array}{c}\text { Severity } \\
--(\%)\end{array}$ & Incidence & $\begin{array}{c}\text { Rating } \\
(0-9)\end{array}$ & $\begin{array}{l}\text { Heading date } \\
\text { (Julian) }\end{array}$ & $\begin{array}{l}\text { Height } \\
(\mathrm{cm})\end{array}$ \\
\hline & & \multicolumn{8}{|c|}{ Population 1 (26R58/VA01W-476//KY97C-0574-01) } \\
\hline & Mean & 19.3 & 20.5 & 42.4 & 52.2 & 78.0 & 5.6 & 130.9 & 81.4 \\
\hline 21 & Max & 57.1 & 56.4 & 95.9 & 95.9 & 100.0 & 9.0 & 145.0 & 99.1 \\
\hline & Min & 7.0 & 8.3 & 11.9 & 21.3 & 30.0 & 1.0 & 126.0 & 66.0 \\
\hline & & \multicolumn{8}{|c|}{ Population $2(25 R 54 / V A 01 W-476 / / K Y 97 C-0574-01)$} \\
\hline & Mean & 15.4 & 18.9 & 41.4 & 50.2 & 80.1 & 5.4 & 131.4 & 85.3 \\
\hline 24 & Max & 32.8 & 37.0 & 86.3 & 86.3 & 100.0 & 8.0 & 147.0 & 109.2 \\
\hline & Min & 5.9 & 5.0 & 8.5 & 23.7 & 30.0 & 1.0 & 124.0 & 66.0 \\
\hline & & \multicolumn{8}{|c|}{ Population 3 (25R54/VA01W-476//KY97C-0554-02) } \\
\hline & Mean & 16.5 & 15.3 & 34.8 & 45.5 & 71.4 & 5.9 & 130.4 & 84.0 \\
\hline 36 & Max & 60.8 & 42.5 & 89.2 & 89.2 & 100.0 & 9.0 & 137.0 & 101.6 \\
\hline & Min & 3.7 & 3.5 & 4.7 & 15.9 & 20.0 & 2.0 & 125.0 & 66.0 \\
\hline & & \multicolumn{8}{|c|}{ Population $4(25 R 78 / V A 01 W-476)$} \\
\hline & Mean & 18.4 & 14.1 & 33.7 & 47.5 & 69.4 & 5.8 & 128.7 & 76.5 \\
\hline 45 & $\operatorname{Max}$ & 70.3 & 34.6 & 93.5 & 93.5 & 100.0 & 9.0 & 133.0 & 94.0 \\
\hline & Min & 2.7 & 3.5 & 0.2 & 4.3 & 5.0 & 1.0 & 122.0 & 58.4 \\
\hline & & \multicolumn{8}{|c|}{ Population 5 (KY93C-1238-17-1/VA01W-476) } \\
\hline & Mean & 12.5 & 12.9 & 27.8 & 41.8 & 63.2 & 5.6 & 128.9 & 83.6 \\
\hline 29 & Max & 37.1 & 33.4 & 85.3 & 85.3 & 100.0 & 8.0 & 143.0 & 106.7 \\
\hline & Min & 2.1 & 3.5 & 5.5 & 13.2 & 20.0 & 1.0 & 122.0 & 61.0 \\
\hline
\end{tabular}


Table A.3.1 (continued): Mean, Maximum (Max) and Minimum (Min) Fusarium damaged kernels (FDK), deoxynivalenol level (DON), FHB index, severity, incidence, FHB rating, heading date and plant height for five wheat populations and their different parents in the Lexington, KY scab nursery 2010 and 2011.

\begin{tabular}{|c|c|c|c|c|c|c|c|c|c|c|c|c|c|c|c|c|}
\hline $\mathbf{N}$ & $\begin{array}{c}\text { FDK } \\
(\%)\end{array}$ & & $\begin{array}{l}\text { DON } \\
(\mathrm{ppm})\end{array}$ & & Index & & Severit & & $\begin{array}{l}\text { Inciden } \\
(\%)\end{array}$ & - & $\begin{array}{c}\text { Rating } \\
(0-9)\end{array}$ & & $\begin{array}{c}\text { Headin } \\
\vdots \\
\text { (Julian) }\end{array}$ & & $\begin{array}{r}\text { Height } \\
(\mathrm{cm})\end{array}$ & \\
\hline $25 \mathrm{R} 54$ & 19.8 & (2.8) & 20.6 & $(2.8)$ & 44.9 & (7.14) & 56.8 & (5.7) & 76.5 & (6.7) & 7.1 & (0.6) & 129.4 & $(0.8)$ & 82.3 & $(1.3)$ \\
\hline $25 \mathrm{R} 78$ & 24.9 & $(4.5)$ & 22.8 & (4.4) & 41.8 & (11.3) & 56.2 & $(9.1)$ & 77.5 & (10.6) & 6.3 & $(0.9)$ & 129.5 & (1.3) & 77.5 & (2.0) \\
\hline $26 \mathrm{R} 58$ & 24.7 & $(4.5)$ & 26.8 & (4.4) & 57.5 & (11.3) & 65.5 & $(9.1)$ & 86.3 & (10.6) & 6.5 & $(0.9)$ & 131.0 & $(1.3)$ & 77.5 & (2.0) \\
\hline KY93C-1238-17-1 & 21.7 & (4.5) & 22.8 & (4.4) & 65.6 & (11.3) & 60.5 & $(9.1)$ & 64.8 & (10.6) & 6.3 & $(0.9)$ & 130.3 & (1.3) & 84.5 & (2.0) \\
\hline KY97C-0554-02 & 19.1 & $(4.5)$ & 24.6 & (5.1) & 53.2 & (11.3) & 57.0 & $(9.1)$ & 90.0 & (10.6) & 7.8 & $(0.9)$ & 130.8 & (1.3) & 83.2 & (2.0) \\
\hline KY97C-0574-01 & 19.2 & $(3.2)$ & 30.2 & (3.1) & 58.0 & (8.0) & 61.4 & (6.4) & 95.0 & (7.5) & 6.6 & $(0.7)$ & 132.1 & $(0.9)$ & 84.1 & (1.4) \\
\hline VA01W-476 & 9.7 & (2.1) & 5.5 & (2.1) & 29.0 & (5.8) & 44.2 & (4.7) & 60.4 & (5.5) & 5.4 & $(0.4)$ & 127.0 & (0.6) & 72.7 & (0.9) \\
\hline
\end{tabular}

Numbers in parentheses are standard errors (SE) 
Table A.3.2: Mean, Maximum (Max) and Minimum (Min) Fusarium damaged kernels (FDK), deoxynivalenol level (DON), FHB index, severity, incidence, FHB rating, heading date and plant height for five wheat populations and their different parents in the Lexington, KY scab nursery 2010.

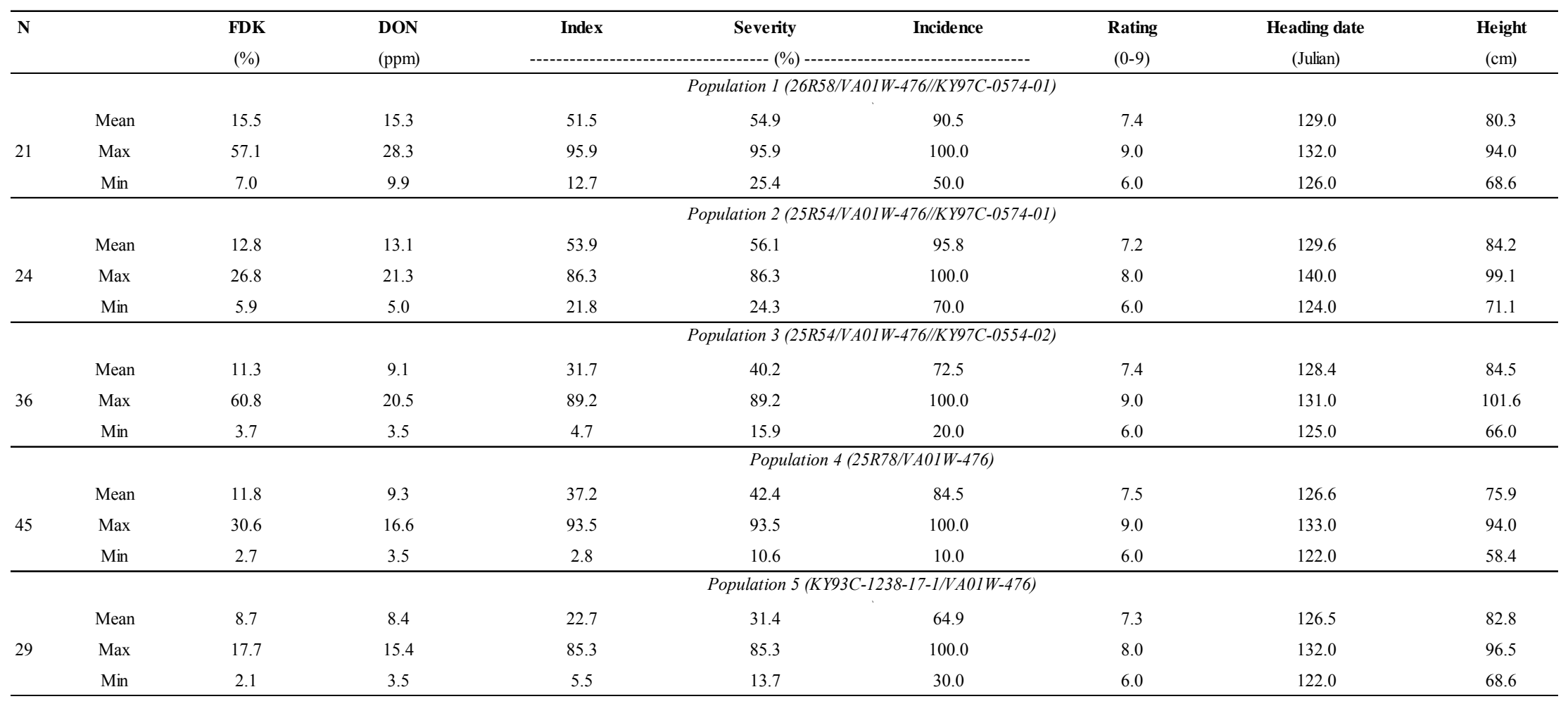


Table A.3.2 (continued): Mean, Maximum (Max) and Minimum (Min) Fusarium damaged kernels (FDK), deoxynivalenol level (DON), FHB index, severity, incidence, FHB rating, heading date and plant height for five wheat populations and their different parents in the Lexington, KY scab nursery 2010.

\begin{tabular}{|c|c|c|c|c|c|c|c|c|c|c|c|c|c|c|c|c|}
\hline & $\begin{array}{c}\text { FDK } \\
(\%)\end{array}$ & & $\begin{array}{l}\text { DON } \\
\text { (ppm) }\end{array}$ & & Index & & Severity & 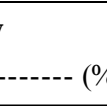 & Incidenc & & $\begin{array}{c}\text { Rating } \\
(0-9)\end{array}$ & & $\begin{array}{c}\text { Heading } \\
\text { (Julian) }\end{array}$ & & $\begin{array}{c}\text { Height } \\
(\mathrm{cm})\end{array}$ & \\
\hline $25 \mathrm{R} 54$ & 15.7 & $(1.7)$ & 13.2 & $(1.4)$ & 55.3 & $(10.4)$ & 64.1 & $(9.2)$ & 85.0 & (9.6) & 8.2 & $(0.3)$ & 127.2 & $(0.7)$ & 81.3 & $(1.4)$ \\
\hline $25 \mathrm{R} 78$ & 18.7 & (2.9) & 14.1 & $(2.4)$ & 48.3 & $(18.0)$ & 48.3 & $(15.8)$ & 100.0 & $(16.6)$ & 8.0 & $(0.5)$ & 127.5 & $(1.2)$ & 76.2 & $(2.5)$ \\
\hline $26 \mathrm{R} 58$ & 21.2 & $(2.9)$ & 23.1 & $(2.4)$ & 73.1 & $(18.0)$ & 73.1 & $(15.8)$ & 100.0 & $(16.6)$ & 8.0 & $(0.5)$ & 129.0 & $(1.2)$ & 76.2 & $(2.5)$ \\
\hline KY93C-1238-17-1 & 13.3 & $(2.9)$ & 15.6 & $(2.4)$ & 57.5 & $(18.0)$ & 62.2 & $(15.8)$ & 90.0 & $(16.6)$ & 8.0 & $(0.5)$ & 128.5 & $(1.2)$ & 81.3 & $(2.5)$ \\
\hline KY97C-0554-02 & 8.6 & (2.9) & 13.4 & (3.4) & 39.3 & $(18.0)$ & 46.9 & $(15.8)$ & 80.0 & $(16.6)$ & 8.0 & $(0.5)$ & 128.5 & $(1.2)$ & 82.6 & $(2.5)$ \\
\hline KY97C-0574-01 & 13.3 & $(2.0)$ & 18.2 & $(1.7)$ & 61.8 & $(12.7)$ & 61.8 & $(11.2)$ & 100.0 & $(11.7)$ & 7.8 & $(0.3)$ & 130.3 & $(1.2)$ & 81.9 & (1.8) \\
\hline VA01W-476 & 6.5 & (1.3) & 3.6 & $(1.1)$ & 28.4 & (9.6) & 39.5 & $(8.44)$ & 64.3 & (8.9) & 7.3 & $(0.2)$ & 125.2 & $(1.2)$ & 72.4 & (1.1) \\
\hline
\end{tabular}

Numbers in parentheses are standard errors (SE) 
Table A.3.3: Mean, Maximum (Max) and Minimum (Min) Fusarium damaged kernels (FDK), deoxynivalenol level (DON), FHB index, severity, incidence, FHB rating, heading date and plant height for five wheat populations and their different parents in the Lexington, KY scab nursery 2011.

\begin{tabular}{|c|c|c|c|c|c|c|c|c|c|}
\hline $\mathbf{N}$ & & $\begin{array}{l}\text { FDK } \\
(\%)\end{array}$ & $\begin{array}{l}\text { DON } \\
\text { (ppm) }\end{array}$ & Index & $\begin{array}{l}\text { Severity } \\
----(\%)\end{array}$ & Incidence & $\begin{array}{c}\text { Rating } \\
(0-9)\end{array}$ & $\begin{array}{l}\text { Heading date } \\
\text { (Julian) }\end{array}$ & $\begin{array}{l}\text { Height } \\
(\mathrm{cm})\end{array}$ \\
\hline & & \multicolumn{8}{|c|}{ Population 1 (26R58/VA01W-476//KY97C-0574-01) } \\
\hline & Mean & 23.0 & 25.8 & 33.2 & 49.4 & 65.5 & 3.9 & 132.7 & 82.6 \\
\hline \multirow[t]{4}{*}{21} & Max & 46.9 & 56.4 & 72.2 & 81.3 & 100.0 & 7.0 & 145.0 & 99.1 \\
\hline & Min & 9.5 & 8.3 & 11.9 & 21.3 & 30.0 & 1.0 & 129.0 & 66.0 \\
\hline & & \multicolumn{8}{|c|}{ Population $2(25 R 54 / V A 01 W-476 / / K Y 97 C-0574-01)$} \\
\hline & Mean & 17.8 & 24.6 & 29.0 & 44.4 & 64.4 & 3.6 & 133.1 & 86.5 \\
\hline \multirow[t]{4}{*}{24} & Max & 32.8 & 37.0 & 65.0 & 72.4 & 100.0 & 8.0 & 147.0 & 109.2 \\
\hline & Min & 6.1 & 14.0 & 8.5 & 23.7 & 30.0 & 1.0 & 130.0 & 66.0 \\
\hline & & \multicolumn{8}{|c|}{ Population 3 (25R54/VA01W-476//KY97C-0554-02) } \\
\hline & Mean & 21.7 & 21.4 & 38.0 & 50.8 & 70.3 & 4.7 & 132.4 & 83.5 \\
\hline \multirow[t]{4}{*}{36} & $\operatorname{Max}$ & 44.8 & 42.5 & 87.0 & 87.0 & 100.0 & 8.0 & 137.0 & 99.1 \\
\hline & Min & 9.1 & 10.7 & 7.2 & 24.0 & 25.0 & 2.0 & 130.0 & 66.0 \\
\hline & & \multicolumn{8}{|c|}{ Population $4(25 R 78 / V A 01 W-476)$} \\
\hline & Mean & 25.2 & 19.1 & 30.3 & 52.2 & 55.3 & 4.0 & 130.9 & 77.0 \\
\hline \multirow[t]{4}{*}{45} & $\operatorname{Max}$ & 70.3 & 34.6 & 65.8 & 78.5 & 95.0 & 8.0 & 133.0 & 94.0 \\
\hline & Min & 4.4 & 7.7 & 0.2 & 4.3 & 5.0 & 1.0 & 126.0 & 61.0 \\
\hline & & \multicolumn{8}{|c|}{ Population 5 (KY93C-1238-17-1/VA01W-476) } \\
\hline & Mean & 16.3 & 17.3 & 32.8 & 51.6 & 62.0 & 4.0 & 131.2 & 84.5 \\
\hline \multirow[t]{2}{*}{29} & Max & 37.1 & 33.4 & 84.1 & 84.1 & 100.0 & 7.0 & 143.0 & 106.7 \\
\hline & Min & 4.4 & 7.9 & 7.2 & 13.2 & 20.0 & 1.0 & 125.0 & 61.0 \\
\hline
\end{tabular}


Table A.3.3 (continued): Mean, Maximum (Max) and Minimum (Min) Fusarium damaged kernels (FDK), deoxynivalenol level (DON), FHB index, severity, incidence, FHB rating, heading date and plant height for five wheat populations and their different parents in the Lexington, KY scab nursery 2011.

\begin{tabular}{|c|c|c|c|c|c|c|c|c|c|c|c|c|c|c|c|c|}
\hline & $\begin{array}{c}\text { FDK } \\
(\%)\end{array}$ & & $\begin{array}{l}\text { DON } \\
\text { (ppm) }\end{array}$ & & Index & & Severit & $-----\cdot$ & Inciden & & $\begin{array}{l}\text { Rating } \\
(0-9)\end{array}$ & & $\begin{array}{l}\text { Headin } \\
\text { (Julian) }\end{array}$ & & $\begin{array}{l}\text { Heigh } \\
(\mathrm{cm})\end{array}$ & \\
\hline $25 \mathrm{R} 54$ & 25.8 & $(3.2)$ & 31.9 & $(2.8)$ & 29.4 & (5.9) & 45.8 & (4.9) & 63.8 & $(9.5)$ & 5.5 & $(0.5)$ & 132.8 & $(0.7)$ & 83.8 & (1.9) \\
\hline $25 \mathrm{R} 78$ & 31.1 & (4.5) & 31.5 & $(4.0)$ & 35.3 & (8.3) & 64.1 & (6.9) & 55.0 & (13.4) & 4.5 & $(0.8)$ & 131.5 & (1.1) & 78.7 & (2.8) \\
\hline $26 \mathrm{R} 58$ & 28.2 & (4.5) & 30.6 & $(4.0)$ & 42.0 & (8.3) & 57.8 & (6.9) & 72.5 & (13.4) & 5.0 & $(0.8)$ & 133.0 & (1.1) & 78.7 & (2.8) \\
\hline KY93C-1238-17-1 & 30.2 & $(4.5)$ & 30 & $(4.0)$ & 41.2 & (8.3) & 58.9 & (6.9) & 67.5 & (13.4) & 4.5 & $(0.8)$ & 132.0 & (1.1) & 87.6 & (2.8) \\
\hline KY97C-0554-02 & 29.6 & (4.5) & 30.2 & $(4.0)$ & 67.2 & (8.3) & 67.2 & (6.9) & 100.0 & (13.4) & 7.5 & $(0.8)$ & 133.0 & (1.1) & 83.8 & (2.8) \\
\hline KY97C-0574-01 & 25 & (3.2) & 42.3 & (2.8) & 54.2 & (5.9) & 61.0 & (4.9) & 90.0 & $(9.5)$ & 5.5 & $(0.5)$ & 134.0 & $(0.7)$ & 86.4 & (1.9) \\
\hline VA01W-476 & 13.7 & (2.2) & 8 & (2.0) & 27.2 & $(4.2)$ & 48.2 & (3.4) & 55.0 & (6.7) & 3.1 & $(0.4)$ & 129.3 & $(0.5)$ & 73.0 & (1.4) \\
\hline
\end{tabular}

Numbers in parentheses are standard errors (SE) 
Table A.3.4: Mean Fusarium damaged kernels (FDK), deoxynivalenol level (DON), FHB index, severity, incidence, FHB rating, heading date and plant height for wheat lines homozygous for resistant (R) and susceptible (S) alleles at Fhb1, Lexington 2010 and 2011.

\begin{tabular}{|c|c|c|c|c|c|c|c|c|c|c|c|c|c|c|c|c|c|}
\hline Fhb1 class & $\mathbf{N}$ & $\begin{array}{c}\text { FDK } \\
(\%) \\
\end{array}$ & & $\begin{array}{l}\text { DON } \\
(\mathrm{ppm})\end{array}$ & & Index & & $\begin{array}{l}\text { Severity } \\
-(\%)--\end{array}$ & $-\cdots--\cdot$ & Incidence & & $\begin{array}{l}\text { Rating } \\
(0-9)\end{array}$ & & $\begin{array}{c}\text { HD } \\
\text { (Julian) }\end{array}$ & & $\begin{array}{c}\text { Height } \\
(\mathrm{cm})\end{array}$ & \\
\hline & & \multicolumn{16}{|c|}{ Population 1 (26R58/VA01W-476//KY97C-0574-01) } \\
\hline $\mathrm{S}$ & 12 & 21.3 & \multirow{2}{*}{ ** } & 21.7 & \multirow{2}{*}{ * } & 40.7 & & 51.6 & & 74.8 & \multirow{2}{*}{ * } & \multirow{2}{*}{\multicolumn{2}{|c|}{$\begin{array}{l}5.6 \\
5.6 \\
\end{array}$}} & 130.5 & \multirow{2}{*}{ * } & 83.1 & \multirow{2}{*}{ ** } \\
\hline $\mathrm{R}$ & 9 & 16.5 & & 19.0 & & 44.6 & & 52.9 & & 82.2 & & & & 131.4 & & 79.2 & \\
\hline & & \multicolumn{16}{|c|}{ Population 2 (25R54/VA01W-476//KY97C-0574-01) } \\
\hline $\mathrm{S}$ & 13 & 17.9 & \multirow{2}{*}{$* *$} & 20.0 & \multirow{2}{*}{$*$} & 46.6 & \multirow{2}{*}{$* *$} & 55.5 & \multirow{2}{*}{$* *$} & 81.4 & & 5.7 & \multirow{2}{*}{$* *$} & 132.1 & \multirow{2}{*}{$* *$} & 86.8 & \multirow{2}{*}{ ** } \\
\hline $\mathrm{R}$ & 11 & 12.5 & & 17.7 & & 35.3 & & 43.9 & & 78.5 & & 5.1 & & 130.5 & & 83.6 & \\
\hline & & \multicolumn{16}{|c|}{ Population 3 (25R54/VA01W-476//KY97C-0554-02) } \\
\hline $\mathrm{S}$ & 15 & 20.2 & \multirow{2}{*}{ ** } & 17.7 & \multirow{2}{*}{$* *$} & 41.4 & \multirow{2}{*}{$* *$} & 50.2 & \multirow{2}{*}{$* *$} & 78.5 & \multirow{2}{*}{$* *$} & 6.4 & \multirow{2}{*}{$* *$} & 130.8 & \multirow{2}{*}{ * } & 83.0 & \multirow{2}{*}{$*$} \\
\hline $\mathrm{R}$ & 21 & 13.8 & & 13.6 & & 30.2 & & 42.1 & & 66.4 & & 5.7 & & 130.2 & & 84.8 & \\
\hline & & \multicolumn{16}{|c|}{ Population $4(25 R 78 / V A 01 W-476)$} \\
\hline $\mathrm{S}$ & 24 & 21.9 & \multirow{2}{*}{ ** } & 16.1 & \multirow{2}{*}{$* *$} & 39.3 & \multirow{2}{*}{$* *$} & 52.5 & \multirow{2}{*}{$* *$} & 73.8 & \multirow{2}{*}{ ** } & 6.2 & \multirow{2}{*}{$* *$} & \multirow{2}{*}{\multicolumn{2}{|c|}{$\begin{array}{l}128.9 \\
128.6\end{array}$}} & 76.5 & \\
\hline $\mathrm{R}$ & 21 & 14.4 & & 11.9 & & 27.5 & & 42.0 & & 64.6 & & 5.3 & & & & 76.5 & \\
\hline & & & & & & & & oulation 5 & KY93 & $238-17-1 / V$ & $401 W$ & & & & & & \\
\hline $\mathrm{S}$ & 15 & 15.8 & $* *$ & 14.8 & $* *$ & 32.7 & $* *$ & 45.6 & $* *$ & 69.8 & $* *$ & 5.9 & ** & 129.2 & * & 84.8 & $*$ \\
\hline $\mathrm{R}$ & 14 & 9.0 & & 10.8 & & 22.8 & & 37.8 & & 56.8 & & 5.4 & & 128.5 & & 82.5 & \\
\hline
\end{tabular}

(Differences between $\mathrm{S}$ and $\mathrm{R}$ classes significant at $\mathrm{P}<0.05,0.01$, respectively) 
Table A.3.5: Mean Fusarium damaged kernels (FDK), deoxynivalenol level (DON), FHB index, severity, incidence, FHB rating, heading date and plant height for wheat lines homozygous for resistant (R) and susceptible (S) alleles at Fhb1, Lexington 2010.

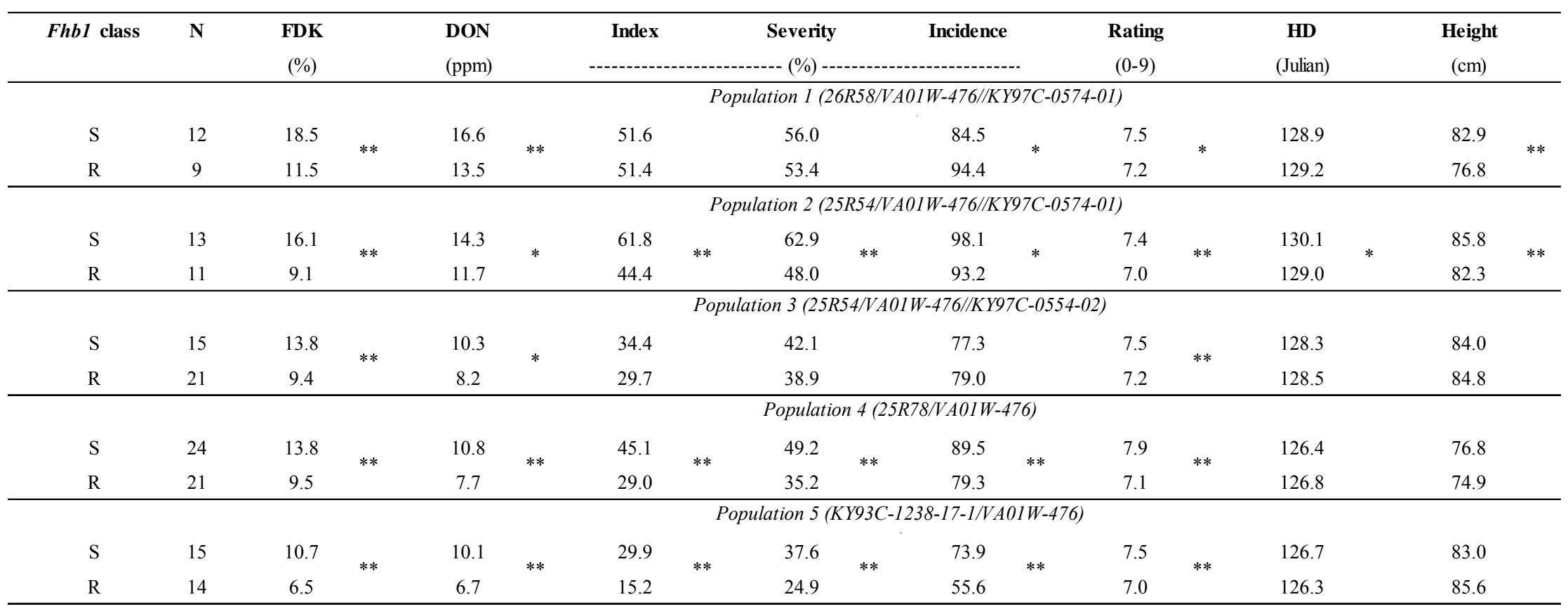

*,** (Differences between $\mathrm{S}$ and $\mathrm{R}$ classes significant at $\mathrm{P}<0.05,0.01$, respectively) 
Table A.3.6: Mean Fusarium damaged kernels (FDK), deoxynivalenol level (DON), FHB index, severity, incidence, FHB rating, heading date and plant height for wheat lines homozygous for resistant (R) and susceptible (S) alleles at Fhb1, Lexington 2011.

\begin{tabular}{|c|c|c|c|c|c|c|c|c|c|c|c|c|c|c|}
\hline Fhb1 class & $\mathbf{N}$ & $\begin{array}{c}\text { FDK } \\
(\%)\end{array}$ & & $\begin{array}{l}\text { DON } \\
(\mathrm{ppm})\end{array}$ & & Index & $\begin{array}{r}\text { Severity } \\
---(\%)\end{array}$ & Incidence & $\begin{array}{c}\text { Rating } \\
(0-9)\end{array}$ & & $\begin{array}{c}\text { HD } \\
\text { (Julian) }\end{array}$ & & $\begin{array}{l}\text { Height } \\
(\mathrm{cm})\end{array}$ & \\
\hline & & \multicolumn{13}{|c|}{ Population 1 (26R58/VA01W-476//KY97C-0574-01) } \\
\hline $\mathrm{S}$ & 12 & 24.1 & & 26.7 & & 37.8 & 52.4 & 62.1 & 4.1 & & 132.0 & \multirow{2}{*}{$* *$} & 83.3 & \\
\hline $\mathrm{R}$ & 9 & 21.5 & & 24.5 & & 29.8 & 47.2 & 70.0 & 3.8 & & 133.6 & & 81.7 & \\
\hline & & \multicolumn{13}{|c|}{ Population 2 (25R54/VA01W-476//KY97C-0574-01) } \\
\hline $\mathrm{S}$ & 13 & 19.6 & \multirow{2}{*}{ * } & 25.4 & & 31.4 & 48.1 & 64.8 & 3.9 & \multirow{2}{*}{ * } & 134.0 & \multirow{2}{*}{$* *$} & 87.7 & \\
\hline $\mathrm{R}$ & 11 & 15.8 & & 23.7 & & 26.2 & 39.9 & 63.9 & 3.2 & & 132.1 & & 85.0 & \\
\hline & & \multicolumn{13}{|c|}{ Population $3(25 R 54 / V A 01 W-476 / / K Y 97 C-0554-02)$} \\
\hline $\mathrm{S}$ & 15 & 26.6 & \multirow{2}{*}{ ** } & 25.0 & \multirow{2}{*}{$* *$} & 48.6 & 58.7 & 79.7 & \multirow{2}{*}{$* *$} & \multirow{2}{*}{$* *$} & 133.2 & \multirow{2}{*}{$* *$} & 82.0 & \multirow{2}{*}{ * } \\
\hline $\mathrm{R}$ & 21 & 18.1 & & 18.9 & & 30.7 & 45.3 & 63.8 & & & 131.9 & & 84.7 & \\
\hline$\infty$ & & & & & & & Populat & on $4(25 R 78 / V A 01 W$ & $476)$ & & & & & \\
\hline $\mathrm{S}$ & 24 & 30.3 & \multirow{2}{*}{$* *$} & 21.6 & \multirow{2}{*}{$* *$} & 34.1 & 55.3 & 59.8 & \multirow{2}{*}{$* *$} & \multirow{2}{*}{$* *$} & 130.8 & & \multirow{2}{*}{\multicolumn{2}{|c|}{$\begin{array}{l}76.1 \\
78.1 \\
\end{array}$}} \\
\hline $\mathrm{R}$ & 21 & 19.4 & & 16.2 & & 26.1 & 48.6 & 50.2 & & & 130.9 & & & \\
\hline & & \multicolumn{13}{|c|}{ Population 5 (KY93C-1238-17-1/VA01W-476) } \\
\hline $\mathrm{S}$ & 15 & 20.9 & \multirow{2}{*}{$* *$} & 19.6 & \multirow{2}{*}{$* *$} & 35.3 & 52.9 & 65.9 & 4.2 & & 131.7 & \multirow{2}{*}{$* *$} & 86.5 & \multirow{2}{*}{ ** } \\
\hline $\mathrm{R}$ & 14 & 11.5 & & 14.8 & & 30.2 & 50.3 & 58.0 & 3.7 & & 130.7 & & 82.4 & \\
\hline
\end{tabular}

*, ** (Differences between $\mathrm{S}$ and $\mathrm{R}$ classes significant at $\mathrm{P}<0.05,0.01$, respectively) 
Table A.3.7: Mean Fusarium damaged kernels (FDK), deoxynivalenol level (DON), FHB index, severity, incidence, FHB rating, heading date and plant height for homozygous susceptible (S), resistant (R) and heterozygous $(\mathrm{H})$ wheat lines at $Q F h s . n a u-2 D L$, Lexington 2010 and 2011.

\begin{tabular}{|c|c|c|c|c|c|c|c|c|c|c|c|c|c|c|c|c|c|}
\hline \multirow[t]{2}{*}{$2 D L$ class } & \multirow[t]{2}{*}{$\mathbf{N}$} & \multicolumn{2}{|l|}{$\begin{array}{c}\text { FDK } \\
(\%)\end{array}$} & \multicolumn{2}{|l|}{$\begin{array}{l}\text { DON } \\
(\mathrm{ppm})\end{array}$} & \multicolumn{2}{|l|}{ Index } & \multicolumn{2}{|c|}{ Severity } & Incidence & & \multicolumn{2}{|c|}{$\begin{array}{c}\text { Rating } \\
(0-9)\end{array}$} & \multicolumn{2}{|l|}{$\begin{array}{c}\text { HD } \\
\text { (Julian) }\end{array}$} & \multicolumn{2}{|c|}{$\begin{array}{c}\text { Height } \\
(\mathrm{cm})\end{array}$} \\
\hline & & \multicolumn{16}{|c|}{ Population 1 (26R58/VA01W-476//KY97C-0574-01) } \\
\hline $\mathrm{S}$ & 9 & 21.9 & $\mathrm{~b}$ & 21.9 & $\mathrm{~b}$ & 47.0 & $\mathrm{~b}$ & 55.9 & $\mathrm{~b}$ & 81.7 & a & 6.0 & $\mathrm{c}$ & 131.1 & a & 79.1 & a \\
\hline $\mathrm{H}$ & 8 & 16.3 & a & 18.3 & a & 37.3 & a & 48.1 & a & 75.8 & a & 5.5 & $\mathrm{~b}$ & 130.5 & a & 82.7 & $\mathrm{~b}$ \\
\hline \multirow[t]{2}{*}{$\mathrm{R}$} & 2 & 16.7 & a & 16.7 & a & 40.3 & $\mathrm{ab}$ & 49.4 & $\mathrm{ab}$ & 73.1 & a & 4.5 & a & 131.5 & a & 84.1 & $\mathrm{~b}$ \\
\hline & & \multicolumn{16}{|c|}{ Population $2(25 R 54 / V A 01 W-476 / / K Y 97 C-0574-01)$} \\
\hline S & 4 & 14.5 & a & 20.6 & $\mathrm{~b}$ & 37.1 & a & 48.7 & a & 77.2 & a & 5.1 & a & 130.9 & a & 85.4 & a \\
\hline $\mathrm{H}$ & 15 & 15.6 & a & 19.3 & $\mathrm{~b}$ & 41.7 & a & 50.0 & a & 80.4 & a & 5.4 & $\mathrm{ab}$ & 132.1 & $\mathrm{~b}$ & 85.4 & a \\
\hline \multirow[t]{2}{*}{$\mathrm{R}$} & 4 & 14.1 & a & 15.0 & a & 42.1 & $\mathrm{a}$ & 49.4 & a & 82.5 & a & 5.8 & $\mathrm{~b}$ & 129.9 & a & 83.8 & $\mathrm{a}$ \\
\hline & & \multicolumn{16}{|c|}{ Population 3 (25R54/VA01W-476//KY97C-0554-02) } \\
\hline S & 17 & 15.0 & a & 14.7 & a & 33.2 & $\mathrm{ab}$ & 45.1 & $a b$ & 68.4 & a & 5.9 & a & 130.0 & a & 84.1 & a \\
\hline $\mathrm{H}$ & 9 & 18.6 & $\mathrm{~b}$ & 15.8 & a & 38.1 & $\mathrm{~b}$ & 47.4 & $\mathrm{~b}$ & 76.9 & $\mathrm{~b}$ & 6.1 & a & 130.7 & $\mathrm{~b}$ & 83.2 & a \\
\hline \multirow[t]{2}{*}{$\mathrm{R}$} & 7 & 16.2 & $\mathrm{ab}$ & 15.4 & a & 29.3 & a & 40.2 & a & 66.4 & a & 5.8 & a & 130.7 & b & 85.0 & a \\
\hline & & \multicolumn{16}{|c|}{ Population $4(25 R 78 / V A 01 W-476)$} \\
\hline $\mathrm{S}$ & 12 & 21.9 & $\mathrm{~b}$ & 16.3 & $\mathrm{~b}$ & 35.3 & a & 48.7 & a & 71.4 & a & 5.9 & a & 128.8 & a & 74.8 & a \\
\hline $\mathrm{H}$ & 20 & 16.6 & a & 13.2 & a & 31.5 & a & 46.0 & a & 66.7 & a & 5.7 & a & 128.6 & $\mathrm{a}$ & 76.7 & $\mathrm{ab}$ \\
\hline \multirow[t]{2}{*}{$\mathrm{R}$} & 7 & 14.3 & a & 12.7 & a & 34.5 & a & 47.8 & a & 71.4 & a & 5.6 & a & 128.6 & a & 78.7 & $\mathrm{~b}$ \\
\hline & & \multicolumn{16}{|c|}{ Population 5 (KY93C-1238-17-1/VA01W-476) } \\
\hline S & 6 & 11.4 & a & 13.3 & $\mathrm{~b}$ & 23.5 & a & 41.0 & a & 55.2 & a & 5.5 & $\mathrm{ab}$ & 129.1 & $\mathrm{~b}$ & 83.9 & $\mathrm{ab}$ \\
\hline $\mathrm{H}$ & 15 & 14.0 & $\mathrm{~b}$ & 14.0 & $\mathrm{~b}$ & 31.0 & $\mathrm{~b}$ & 43.2 & a & 69.1 & $\mathrm{~b}$ & 5.8 & $\mathrm{~b}$ & 129.1 & $\mathrm{~b}$ & 84.7 & $\mathrm{~b}$ \\
\hline $\mathrm{R}$ & 8 & 10.5 & $\mathrm{a}$ & 10.5 & $\mathrm{a}$ & 25.1 & $a b$ & 39.0 & a & 59.2 & $\mathrm{a}$ & 5.4 & $\mathrm{a}$ & 128.3 & $\mathrm{a}$ & 81.5 & $\mathrm{a}$ \\
\hline
\end{tabular}

Means (by population) within the same column followed by different letters are significantly different at $\mathrm{P}<0.05$ 
Table A.3.8: Mean Fusarium damaged kernels (FDK), deoxynivalenol level (DON), FHB index, severity, incidence, FHB rating, heading date and plant height for homozygous susceptible (S), resistant (R) and heterozygous $(\mathrm{H})$ wheat lines at $Q F h s . n a u-2 D L$, Lexington 2010.

\begin{tabular}{|c|c|c|c|c|c|c|c|c|c|c|c|c|c|c|c|c|c|}
\hline $2 D L$ class & $\mathbf{N}$ & $\begin{array}{c}\text { FDK } \\
(\%)\end{array}$ & & \multicolumn{2}{|l|}{$\begin{array}{l}\text { DON } \\
(\mathrm{ppm})\end{array}$} & \multicolumn{2}{|l|}{ Index } & Severity & --- & Incidence & & \multicolumn{2}{|c|}{$\begin{array}{l}\text { Rating } \\
(0-9)\end{array}$} & \multicolumn{2}{|l|}{$\begin{array}{c}\text { HD } \\
\text { (Julian) }\end{array}$} & \multicolumn{2}{|l|}{$\begin{array}{l}\text { Height } \\
(\mathrm{cm})\end{array}$} \\
\hline & & \multicolumn{16}{|c|}{ Population 1 (26R58/VA01W-476//KY97C-0574-01) } \\
\hline $\mathrm{S}$ & 9 & 18.4 & $\mathrm{~b}$ & 15.1 & a & 53.0 & a & 56.5 & a & 91.1 & $\mathrm{ab}$ & 7.8 & $\mathrm{~b}$ & 128.9 & a & 77.6 & a \\
\hline $\mathrm{H}$ & 8 & 12.2 & a & 14.6 & a & 42.6 & a & 47.4 & a & 85.6 & a & 6.9 & a & 128.9 & a & 82.1 & $\mathrm{~b}$ \\
\hline $\mathrm{R}$ & 2 & 13.3 & a & 12.6 & a & 66.2 & a & 66.2 & a & 100.0 & $\mathrm{~b}$ & 7.0 & a & 130.3 & $\mathrm{~b}$ & 81.3 & $\mathrm{~b}$ \\
\hline & & \multicolumn{16}{|c|}{ Population $2(25 R 54 / V A 01 W-476 / / K Y 97 C-0574-01)$} \\
\hline $\mathrm{S}$ & 4 & 11.9 & $\mathrm{ab}$ & 15.5 & $\mathrm{~b}$ & 45.0 & a & 48.3 & a & 95.0 & a & 6.9 & a & 129.9 & $\mathrm{~b}$ & 83.8 & a \\
\hline $\mathrm{H}$ & 15 & 13.5 & $\mathrm{~b}$ & 13.3 & $\mathrm{~b}$ & 53.8 & a & 55.9 & a & 95.7 & a & 7.2 & $\mathrm{~b}$ & 130.1 & $\mathrm{~b}$ & 84.3 & a \\
\hline $\mathrm{R}$ & 4 & 10.5 & a & 8.9 & a & 55.8 & a & 58.0 & a & 96.3 & a & 7.6 & c & 128.1 & a & 83.2 & a \\
\hline & & \multicolumn{16}{|c|}{ Population 3 (25R54/VA01W-476//KY97C-0554-02) } \\
\hline $\mathrm{S}$ & 17 & 9.7 & a & 9.2 & a & 32.8 & $\mathrm{~b}$ & 42.5 & $\mathrm{~b}$ & 71.2 & $\mathrm{ab}$ & 7.4 & $\mathrm{ab}$ & 128.4 & a & 84.3 & a \\
\hline $\mathrm{H}$ & 9 & 13.4 & $\mathrm{~b}$ & 9.2 & a & 37.3 & $\mathrm{~b}$ & 43.6 & $\mathrm{~b}$ & 81.7 & $\mathrm{~b}$ & 7.5 & $\mathrm{~b}$ & 128.6 & a & 84.4 & a \\
\hline $\mathrm{R}$ & 7 & 11.7 & $\mathrm{ab}$ & 8.9 & a & 20.2 & a & 29.4 & a & 61.4 & a & 7.1 & a & 128.4 & a & 85.5 & a \\
\hline & & \multicolumn{16}{|c|}{ Population $4(25 R 78 / V A 01 W-476)$} \\
\hline $\mathrm{S}$ & 12 & 13.5 & c & 10.8 & $\mathrm{~b}$ & 40.7 & a & 44.6 & a & 87.9 & $\mathrm{~b}$ & 7.6 & a & 126.9 & a & 74.8 & a \\
\hline $\mathrm{H}$ & 20 & 11.3 & $\mathrm{~b}$ & 8.8 & a & 33.7 & a & 41.4 & a & 78.7 & a & 7.5 & $\mathrm{a}$ & 126.4 & $\mathrm{a}$ & 75.4 & a \\
\hline $\mathrm{R}$ & 7 & 8.3 & a & 8.6 & a & 39.9 & a & 43.2 & a & 88.1 & $\mathrm{ab}$ & 7.5 & a & 126.4 & a & 78.4 & $\mathrm{~b}$ \\
\hline & & \multicolumn{16}{|c|}{ Population 5 (KY93C-1238-17-1/VA01W-476) } \\
\hline $\mathrm{S}$ & 6 & 8.6 & $\mathrm{ab}$ & 8.0 & $\mathrm{ab}$ & 14.5 & a & 23.3 & a & 56.5 & a & 6.9 & a & 127.1 & $\mathrm{~b}$ & 83.2 & a \\
\hline $\mathrm{H}$ & 15 & 9.3 & $\mathrm{~b}$ & 9.2 & $\mathrm{~b}$ & 26.7 & $\mathrm{~b}$ & 35.0 & $\mathrm{~b}$ & 70.3 & $\mathrm{~b}$ & 7.4 & $\mathrm{~b}$ & 126.6 & $a b$ & 83.1 & a \\
\hline $\mathrm{R}$ & 8 & 7.5 & $\mathrm{a}$ & 7.4 & $\mathrm{a}$ & 21.6 & $\mathrm{ab}$ & 30.9 & $a b$ & 62.0 & $\mathrm{ab}$ & 7.4 & $\mathrm{~b}$ & 126.0 & $\mathrm{a}$ & 81.9 & a \\
\hline
\end{tabular}

Means (by population) within the same column followed by different letters are significantly different at $\mathrm{P}<0.05$ 
Table A.3.9: Mean Fusarium damaged kernels (FDK), deoxynivalenol level (DON), FHB index, severity, incidence, FHB rating, heading date and plant height for homozygous susceptible (S), resistant (R) and heterozygous (H) wheat lines at $Q F h s . n a u-2 D L$, Lexington 2011.

\begin{tabular}{|c|c|c|c|c|c|c|c|c|c|c|c|c|c|c|c|c|c|}
\hline $2 D L$ class & $\mathbf{N}$ & \multicolumn{2}{|l|}{$\begin{array}{c}\text { FDK } \\
(\%)\end{array}$} & \multicolumn{2}{|l|}{$\begin{array}{l}\text { DON } \\
(\mathrm{ppm})\end{array}$} & \multicolumn{2}{|l|}{ Index } & \multicolumn{2}{|c|}{ Severity } & \multicolumn{2}{|c|}{ Incidence } & \multicolumn{2}{|l|}{$\begin{array}{l}\text { Rating } \\
(0-9)\end{array}$} & \multicolumn{2}{|l|}{$\begin{array}{c}\text { HD } \\
\text { (Julian) }\end{array}$} & \multicolumn{2}{|l|}{$\begin{array}{l}\text { Heigth } \\
(\mathrm{cm})\end{array}$} \\
\hline & & \multicolumn{16}{|c|}{ Population 1 (26R58/VA01W-476//KY97C-0574-01) } \\
\hline $\mathrm{S}$ & 9 & 25.4 & $\mathrm{~b}$ & 28.7 & $\mathrm{~b}$ & 41.0 & $\mathrm{c}$ & 55.3 & $\mathrm{~b}$ & 72.2 & a & 4.2 & $\mathrm{~b}$ & 133.4 & $\mathrm{~b}$ & 80.6 & a \\
\hline $\mathrm{H}$ & 8 & 20.3 & a & 21.9 & a & 32.0 & $\mathrm{~b}$ & 48.8 & $\mathrm{~b}$ & 65.9 & a & 4.1 & $\mathrm{~b}$ & 132.1 & a & 83.3 & a \\
\hline $\mathrm{R}$ & 2 & 20.0 & $\mathrm{ab}$ & 20.8 & $\mathrm{a}$ & 14.3 & $\mathrm{a}$ & 32.6 & $\mathrm{a}$ & 46.3 & $\mathrm{~b}$ & 2.0 & a & 132.8 & $\mathrm{ab}$ & 87.0 & a \\
\hline & & \multicolumn{16}{|c|}{ Population $2(25 R 54 / V A 01 W-476 / / K Y 97 C-0574-01)$} \\
\hline $\mathrm{S}$ & 4 & 17.2 & a & 25.7 & $\mathrm{~b}$ & 29.2 & a & 49.1 & a & 59.4 & a & 3.3 & a & 131.9 & a & 87.0 & $\mathrm{a}$ \\
\hline $\mathrm{H}$ & 15 & 17.7 & a & 25.3 & $\mathrm{~b}$ & 29.5 & a & 44.0 & a & 65.2 & a & 3.6 & a & 134.0 & $\mathrm{~b}$ & 86.4 & a \\
\hline $\mathrm{R}$ & 4 & 18.2 & a & 21.4 & $\mathrm{a}$ & 28.4 & a & 40.8 & $\mathrm{a}$ & 68.8 & a & 4.0 & a & 131.6 & $\mathrm{a}$ & 84.5 & a \\
\hline & & \multicolumn{16}{|c|}{ Population 3 (25R54/VA01W-476//KY97C-0554-02) } \\
\hline $\mathrm{S}$ & 17 & 20.2 & a & 20.1 & a & 33.7 & a & 47.8 & a & 65.6 & a & 4.5 & a & 131.7 & a & 83.9 & a \\
\hline $\mathrm{H}$ & 9 & 23.9 & $\mathrm{~b}$ & 22.4 & a & 38.9 & a & 51.3 & a & 72.2 & a & 4.7 & a & 132.9 & $\mathrm{~b}$ & 82.0 & a \\
\hline $\mathrm{R}$ & 7 & 20.8 & $\mathrm{ab}$ & 22.0 & $\mathrm{a}$ & 38.5 & $\mathrm{a}$ & 51.1 & $\mathrm{a}$ & 71.4 & $\mathrm{a}$ & 4.4 & a & 133.0 & $\mathrm{~b}$ & 84.5 & $\mathrm{a}$ \\
\hline & & \multicolumn{16}{|c|}{ Population $4(25 R 78 / V A 01 W-476)$} \\
\hline $\mathrm{S}$ & 12 & 29.9 & $\mathrm{~b}$ & 21.6 & $\mathrm{~b}$ & 30.0 & a & 52.7 & $\mathrm{a}$ & 54.8 & a & 4.3 & a & 130.8 & a & 74.8 & a \\
\hline $\mathrm{H}$ & 20 & 22.0 & a & 17.5 & a & 29.6 & a & 50.9 & a & 54.5 & a & 4.0 & a & 130.7 & a & 78.0 & a \\
\hline $\mathrm{R}$ & 7 & 20.2 & a & 16.8 & a & 28.5 & a & 52.0 & a & 54.3 & a & 3.7 & a & 130.8 & a & 79.1 & a \\
\hline & & \multicolumn{16}{|c|}{ Population 5 (KY93C-1238-17-1/VA01W-476) } \\
\hline $\mathrm{S}$ & 6 & 14.1 & a & 18.6 & $\mathrm{~b}$ & 33.2 & a & 58.0 & $\mathrm{~b}$ & 55.8 & $\mathrm{ab}$ & 4.1 & $\mathrm{ab}$ & 131.2 & $\mathrm{ab}$ & 84.7 & $\mathrm{ab}$ \\
\hline $\mathrm{H}$ & 15 & 18.7 & $\mathrm{~b}$ & 18.7 & $\mathrm{~b}$ & 35.7 & $\mathrm{a}$ & 51.0 & $a b$ & 68.5 & $\mathrm{~b}$ & 4.2 & $\mathrm{~b}$ & 131.5 & $\mathrm{~b}$ & 86.3 & $\mathrm{a}$ \\
\hline $\mathrm{R}$ & 8 & 13.5 & a & 13.6 & a & 29.1 & a & 48.1 & $\mathrm{a}$ & 56.6 & $\mathrm{a}$ & 3.4 & a & 130.6 & a & 81.1 & b \\
\hline
\end{tabular}

Means (by population) within the same column followed by different letters are significantly different at $\mathrm{P}<0.05$ 
Table A.3.10: Mean Fusarium damaged kernels (FDK), deoxynivalenol level (DON), FHB index, severity, incidence, FHB rating, heading date and plant height for wheat lines in each QTL combination class, Lexington 2010 and 2011.

\begin{tabular}{|c|c|c|c|c|c|c|c|c|c|c|c|c|c|c|c|c|c|}
\hline \multirow{3}{*}{$\begin{array}{c}\begin{array}{c}F h b 1 \text { and } 2 D L \\
\text { combination class }\end{array} \\
\text { RH }\end{array}$} & \multirow{3}{*}{$\begin{array}{l}\mathbf{N} \\
4 \\
\end{array}$} & \multicolumn{2}{|l|}{$\begin{array}{l}\text { FDK } \\
(\%)\end{array}$} & \multicolumn{2}{|c|}{$\begin{array}{l}\text { DON } \\
\text { (ppm) }\end{array}$} & \multicolumn{2}{|c|}{$\begin{array}{l}\text { Index } \\
-\end{array}$} & \multicolumn{2}{|c|}{ Severity } & \multicolumn{2}{|c|}{ Incidence } & \multicolumn{2}{|c|}{$\begin{array}{c}\text { Rating } \\
(0-9)\end{array}$} & \multicolumn{2}{|l|}{$\begin{array}{c}\text { Heading } \\
\quad \cdot \\
\text { (Julian) }\end{array}$} & \multicolumn{2}{|c|}{$\begin{array}{c}\text { Heigth } \\
(\mathrm{cm})\end{array}$} \\
\hline & & \multicolumn{16}{|c|}{ Population 1 (26R58/VA01W-476//KY97C-0574-01) } \\
\hline & & 15.4 & a & 19 & a & 26 & a & 45 & a & 55.1 & a & 5.3 & $a b c$ & 130.4 & a & 80.8 & $a b c$ \\
\hline RR & 1 & 17 & a & 16.2 & a & 33.4 & ab & 55.4 & $\mathrm{ab}$ & 53.2 & $\mathrm{ab}$ & 4.5 & a & 131.5 & $\mathrm{ab}$ & 75.6 & a \\
\hline RS & 4 & 17.5 & a & 19.7 & a & 43.6 & $\mathrm{~b}$ & 60.2 & $\mathrm{~b}$ & 68.2 & $\mathrm{~b}$ & 6.3 & d & 132.3 & $\mathrm{~b}$ & 78.6 & a \\
\hline SH & 4 & 17.1 & a & 17.6 & a & 29.8 & a & 51.2 & $\mathrm{ab}$ & 53.4 & a & 5.7 & $\mathrm{~cd}$ & 130.5 & a & 84.6 & c \\
\hline SR & 1 & 16.4 & a & 17.2 & a & 27.3 & $\mathrm{ab}$ & 43.4 & a & 59.3 & $\mathrm{ab}$ & 4.7 & a & 131.5 & $\mathrm{ab}$ & 92.7 & d \\
\hline SS & 5 & 25.5 & $\mathrm{~b}$ & 23.6 & $\mathrm{~b}$ & 33.2 & $\mathrm{ab}$ & 52.4 & $\mathrm{ab}$ & 58.2 & $\mathrm{ab}$ & 5.8 & $\mathrm{~cd}$ & 130.2 & a & 79.5 & $\mathrm{ab}$ \\
\hline & & \multicolumn{16}{|c|}{ Population $2(25 R 54 / V A 01$ W-476//KY97C-0574-01) } \\
\hline RH & 7 & 11.5 & $\mathrm{a}$ & 17.4 & bcd & 23.2 & a & 43.1 & a & 51.7 & $\mathrm{a}$ & 4.7 & $\mathrm{a}$ & 131 & $\mathrm{bcd}$ & 86.3 & $\mathrm{~b}$ \\
\hline $\mathrm{RR}$ & 2 & 13.8 & $\mathrm{a}$ & 16.4 & $\mathrm{ab}$ & 25.2 & $\mathrm{ab}$ & 44.1 & $\mathrm{ab}$ & 58.5 & $\mathrm{ab}$ & 5.9 & $\mathrm{~b}$ & 129.5 & a & 80.3 & a \\
\hline RS & 2 & 14.5 & a & 20.0 & bde & 27.8 & $\mathrm{abc}$ & 46.9 & $\mathrm{abc}$ & 58.3 & $\mathrm{ab}$ & 5.6 & $\mathrm{~b}$ & 130 & $\mathrm{ab}$ & 77.8 & a \\
\hline SH & 8 & 19.2 & $\mathrm{~b}$ & 21.0 & e & 38.4 & $\mathrm{c}$ & 56.0 & $\mathrm{c}$ & 66.3 & $\mathrm{~b}$ & 5.9 & $\mathrm{~b}$ & 133.0 & $\mathrm{e}$ & 84.6 & $\mathrm{~b}$ \\
\hline SR & 2 & 14.5 & a & 13.6 & a & 38.3 & $\mathrm{bc}$ & 54.7 & $\mathrm{bc}$ & 66.0 & $\mathrm{~b}$ & 5.8 & $\mathrm{~b}$ & 130.3 & abcd & 87.3 & $\mathrm{~b}$ \\
\hline SS & 2 & 14.5 & a & 21.2 & $\mathrm{e}$ & 23.9 & a & 50.5 & $a b c$ & 46.0 & a & 4.5 & a & 131.8 & de & 93.0 & c \\
\hline & & \multicolumn{16}{|c|}{ Population 3 (25R54/VA01W-476//KY97C-0554-02) } \\
\hline RH & 2 & 15.6 & abcd & 14.8 & $\mathrm{ab}$ & 22 & abc & 39.7 & $\mathrm{abc}$ & 51.3 & abcd & 5.9 & $\mathrm{ab}$ & 130.4 & $\mathrm{ab}$ & 83.5 & $\mathrm{ab}$ \\
\hline RR & 3 & 14.5 & $\mathrm{ab}$ & 14.2 & ab & 14.7 & a & 32.7 & a & 35.1 & $\mathrm{ab}$ & 5.2 & a & 130.7 & $\mathrm{ab}$ & 86.4 & $\mathrm{~b}$ \\
\hline RS & 14 & 12.8 & a & 12.8 & a & 21.6 & $\mathrm{ab}$ & 42.4 & $\mathrm{~b}$ & 45.9 & $\mathrm{abc}$ & 5.6 & $\mathrm{a}$ & 129.9 & a & 84.5 & $\mathrm{ab}$ \\
\hline SH & 7 & 19.5 & d & 16.1 & $\mathrm{~b}$ & 31.0 & $\mathrm{c}$ & 49.7 & $\mathrm{~cd}$ & 55.8 & d & 6.1 & $\mathrm{~b}$ & 130.8 & $\mathrm{~b}$ & 83.1 & a \\
\hline SR & 4 & 17.5 & $\mathrm{bcd}$ & 16.4 & $\mathrm{~b}$ & 29.3 & $\mathrm{bc}$ & 45.9 & $\mathrm{bc}$ & 53.9 & $c d$ & 6.2 & b & 130.8 & b & 84.0 & $a b$ \\
\hline SS & 3 & 25.1 & e & 23.9 & c & 43.9 & d & 57.9 & d & 64.7 & d & 7.3 & c & 130.4 & $a b$ & 82.3 & a \\
\hline
\end{tabular}

Means within the same column and within population followed by different letters are significantly different at $\mathrm{P}<0.05$ 
Table A.3.10 (continued): Mean Fusarium damaged kernels (FDK), deoxynivalenol level (DON), FHB index, severity, incidence, FHB rating, heading date and plant height for wheat lines in each QTL combination class, Lexington 2010 and 2011.

\begin{tabular}{|c|c|c|c|c|c|c|c|c|c|c|c|c|c|c|c|c|c|}
\hline \multirow[t]{2}{*}{$\begin{array}{l}F h b 1 \text { and } 2 D L \\
\text { combination class }\end{array}$} & \multirow[t]{2}{*}{$\mathbf{N}$} & \multicolumn{2}{|l|}{$\begin{array}{c}\text { FDK } \\
(\%) \\
\end{array}$} & \multicolumn{2}{|l|}{$\begin{array}{l}\text { DON } \\
(\mathrm{ppm})\end{array}$} & \multicolumn{2}{|c|}{ Index } & $\begin{array}{l}\text { Severity } \\
\text { (\%) - }-\end{array}$ & & \multicolumn{2}{|c|}{ Incidence } & \multicolumn{2}{|c|}{$\begin{array}{c}\text { Rating } \\
(0-9)\end{array}$} & \multicolumn{2}{|l|}{$\begin{array}{c}\text { Heading } \\
\dot{\cdot} \text { (Julian) }\end{array}$} & \multicolumn{2}{|c|}{$\begin{array}{c}\text { Heigth } \\
(\mathrm{cm})\end{array}$} \\
\hline & & \multicolumn{16}{|c|}{ Population $4(25 R 78 / V A 01 W-476)$} \\
\hline $\mathrm{RH}$ & 11 & 14.3 & a & 11.2 & a & 19 & $\mathrm{a}$ & 41.4 & a & 39.2 & a & 5.3 & $\mathrm{a}$ & 128.6 & $\mathrm{ab}$ & 76.4 & $\mathrm{ab}$ \\
\hline $\mathrm{RR}$ & 4 & 13.2 & a & 12.1 & ab & 20.9 & $\mathrm{ab}$ & 45.3 & $\mathrm{ab}$ & 43 & $\mathrm{ab}$ & 5.3 & a & 128.9 & $\mathrm{ab}$ & 78.3 & $\mathrm{~b}$ \\
\hline RS & 5 & 16.6 & $\mathrm{ab}$ & 13.2 & $\mathrm{~b}$ & 17.2 & a & 40.9 & a & 36.7 & a & 5.4 & a & 129.2 & $\mathrm{~b}$ & 74.8 & a \\
\hline $\mathrm{SH}$ & 9 & 19.6 & $\mathrm{~b}$ & 15.6 & $\mathrm{c}$ & 28.0 & bc & 51.8 & $\mathrm{bc}$ & 49.9 & $\mathrm{bc}$ & 6.3 & $\mathrm{~b}$ & 128.5 & $\mathrm{ab}$ & 77.0 & $\mathrm{ab}$ \\
\hline SR & 3 & 15.7 & $\mathrm{ab}$ & 14.5 & bc & 30.5 & $\mathrm{bc}$ & 51.5 & $\mathrm{bc}$ & 54.1 & $\mathrm{bc}$ & 6.0 & $\mathrm{~b}$ & 128.2 & a & 79.0 & $\mathrm{~b}$ \\
\hline SS & 7 & 25.8 & $\mathrm{c}$ & 18.5 & $\mathrm{~d}$ & 33.0 & c & 54.2 & c & 55.6 & c & 6.4 & $\mathrm{~b}$ & 128.5 & $\mathrm{ab}$ & 74.8 & a \\
\hline & & \multicolumn{16}{|c|}{ Population 5 (KY93C-1238-17-1/VA01W-476) } \\
\hline $\mathrm{RH}$ & 5 & 8.1 & $\mathrm{a}$ & 11.9 & c & 26.1 & $\mathrm{ab}$ & 39.8 & $\mathrm{ab}$ & 63.0 & $\mathrm{~b}$ & 5.4 & $\mathrm{a}$ & 128.7 & $\mathrm{ab}$ & 84.2 & $\mathrm{~b}$ \\
\hline $\mathrm{RR}$ & 5 & 8.8 & a & 9.2 & ab & 22.4 & a & 36.3 & a & 56.7 & $\mathrm{ab}$ & 5.3 & $\mathrm{a}$ & 128.7 & $\mathrm{ab}$ & 79.4 & $\mathrm{a}$ \\
\hline RS & 4 & 10.4 & a & 11.4 & $\mathrm{bc}$ & 19.5 & a & 37 & a & 49.7 & a & 5.4 & a & 128.1 & a & 84.1 & $\mathrm{~b}$ \\
\hline SH & 10 & 17.0 & $\mathrm{c}$ & 15.0 & ef & 33.4 & $\mathrm{~b}$ & 45.0 & $\mathrm{~b}$ & 72.2 & $\mathrm{c}$ & 6.0 & $\mathrm{~b}$ & 129.3 & $\mathrm{~b}$ & 84.9 & $\mathrm{~b}$ \\
\hline SR & 3 & 13.4 & $\mathrm{~b}$ & 12.7 & cde & 29.3 & ab & 43.4 & $\mathrm{ab}$ & 62.9 & $\mathrm{bc}$ & 5.6 & $\mathrm{ab}$ & 127.7 & a & 85.1 & $\mathrm{~b}$ \\
\hline SS & 2 & 13.4 & $\mathrm{~b}$ & 17.1 & $\mathrm{f}$ & 33.0 & $\mathrm{ab}$ & 49.4 & $\mathrm{~b}$ & 67.5 & $\mathrm{bc}$ & 5.6 & $\mathrm{ab}$ & 131.1 & $\mathrm{c}$ & 83.5 & $\mathrm{~b}$ \\
\hline
\end{tabular}

Means within the same column and within population followed by different letters are significantly different at $\mathrm{P}<0.05$ 
Table A.3.11: Mean Fusarium damaged kernels (FDK), deoxynivalenol level (DON), FHB index, severity, incidence, FHB rating, heading date and plant height for wheat lines in each QTL combination class when all populations were combined, Lexington 2010 and 2011.

\begin{tabular}{|c|c|c|c|c|c|c|c|c|c|c|c|c|c|c|c|c|c|}
\hline $\begin{array}{c}\text { Fhb1 and } 2 D L \text { combination } \\
\text { class }\end{array}$ & $\mathbf{N}$ & $\begin{array}{c}\text { FDK } \\
(\%)\end{array}$ & & $\begin{array}{l}\text { DON } \\
(\mathrm{ppm})\end{array}$ & & $\begin{array}{l}\text { Index } \\
--\cdot-\end{array}$ & - & $\begin{array}{r}\text { Severity } \\
---(\%)--. .\end{array}$ & & Incidence & & $\begin{array}{c}\text { Rating } \\
(0-9)\end{array}$ & & $\begin{array}{c}\text { HD } \\
\text { (Julian) }\end{array}$ & & $\begin{array}{c}\text { Height } \\
(\mathrm{cm})\end{array}$ & \\
\hline RH & 29 & 12.8 & $\mathrm{a}$ & 14.2 & $\mathrm{c}$ & 30.1 & $\mathrm{a}$ & 41.9 & $\mathrm{a}$ & 69 & $a b$ & 5.2 & $\mathrm{a}$ & 129.6 & $a b$ & 81.2 & $\mathrm{a}$ \\
\hline $\mathrm{RR}$ & 15 & 12.3 & $\mathrm{a}$ & 12.4 & $\mathrm{a}$ & 27.3 & $\mathrm{a}$ & 40.4 & $\mathrm{a}$ & 63.4 & $\mathrm{a}$ & 5.3 & a & 129.4 & a & 80.4 & a \\
\hline RS & 29 & 13.9 & $\mathrm{ab}$ & 14.1 & $\mathrm{c}$ & 32.0 & a & 44.2 & $\mathrm{ab}$ & 67.3 & $\mathrm{a}$ & 5.6 & $\mathrm{~b}$ & 129.9 & $\mathrm{~b}$ & 81.5 & ab \\
\hline SH & 38 & 18.5 & c & 16.9 & b & 39.4 & b & 50.6 & $\mathrm{~cd}$ & 75.7 & c & 6.0 & $\mathrm{c}$ & 130.3 & c & 82.6 & b \\
\hline SR & 13 & 15.6 & $\mathrm{~b}$ & 14.5 & d & 37.6 & $\mathrm{~b}$ & 47.8 & $\mathrm{bc}$ & 74.2 & $\mathrm{bc}$ & 5.8 & $\mathrm{cb}$ & 129.4 & ab & 84.3 & $\mathrm{c}$ \\
\hline SS & 19 & 23.1 & d & 20.8 & $\mathrm{e}$ & 41.9 & b & 53.6 & d & 76.1 & c & 6.1 & $\mathrm{c}$ & 129.9 & $\mathrm{ab}$ & 80.1 & a \\
\hline
\end{tabular}

Means within the same column followed by different letters are significantly different at $\mathrm{P}<0.05$ 
Table A.3.12: Heritabilities and their 90\% confidence intervals in parentheses, based on 2 year ANOVA of five wheat populations. Traits evaluated were: deoxynivalenol level (DON), Fusarium damaged kernels (FDK), FHB rating, FHB index, severity and incidence in the Lexington, KY scab nursery 2010 and 2011.

\begin{tabular}{ccccccccccccccccc}
\hline & \multicolumn{2}{c}{$\begin{array}{c}\text { DON } \\
(\mathrm{ppm})\end{array}$} & \multicolumn{2}{c}{$\begin{array}{c}\text { FDK } \\
(\%)\end{array}$} & \multicolumn{2}{c}{ Rating } & \multicolumn{3}{c}{ Index } & \multicolumn{3}{c}{ Severity } & \multicolumn{2}{c}{ Incidence } \\
\hline Overall & 0.75 & $(0.8-0.7)$ & 0.65 & $(0.7-0.6)$ & 0.52 & $(0.6-0.4)$ & 0.33 & $(0.5-0.2)$ & 0.32 & $(0.5-0.2)$ & 0.34 & $(0.5-0.2)$ \\
Population 1 & 0.71 & $(0.8-0.5)$ & 0.82 & $(0.9-0.7)$ & 0.15 & $(0.5--0.5)$ & 0.51 & $(0.7-0.1)$ & 0.51 & $(0.7-0.1)$ & 0.36 & $(0.6--0.1)$ \\
Population 2 & 0.63 & $(0.8-0.4)$ & 0.60 & $(0.8-0.3)$ & 0.59 & $(0.8-0.3)$ & 0.26 & $(0.6--0.3)$ & 0.36 & $(0.7--0.1)$ & 0.49 & $(0.7--0.1)$ \\
Population 3 & 0.59 & $(0.7-0.4)$ & 0.63 & $(0.8-0.4)$ & 0.40 & $(0.6-0.1)$ & 0.41 & $(0.6-0.1)$ & 0.48 & $(0.7-0.2)$ & 0.28 & $(0.5-0.1)$ \\
Population 4 & 0.75 & $(0.8-0.6)$ & 0.58 & $(0.7-0.4)$ & 0.75 & $(0.8-0.6)$ & 0.59 & $(0.7-0.4)$ & 0.63 & $(0.8-0.5)$ & 0.60 & $(0.7-0.4)$ \\
Population 5 & 0.59 & $(0.8-0.3)$ & 0.67 & $(0.8-0.5)$ & 0.40 & $(0.6-0.0)$ & 0.66 & $(0.8-0.4)$ & 0.44 & $(0.7-0.1)$ & 0.66 & $(0.8-0.4)$ \\
\hline
\end{tabular}


Table A.3.13: Genotype mean Pearson correlation coefficients between Fusarium damaged kernels (FDK), deoxynivalenol level (DON), FHB rating, severity, incidence, FHB index, heading date and plant height, in Lexington, KY scab nursery 2010 and 2011.

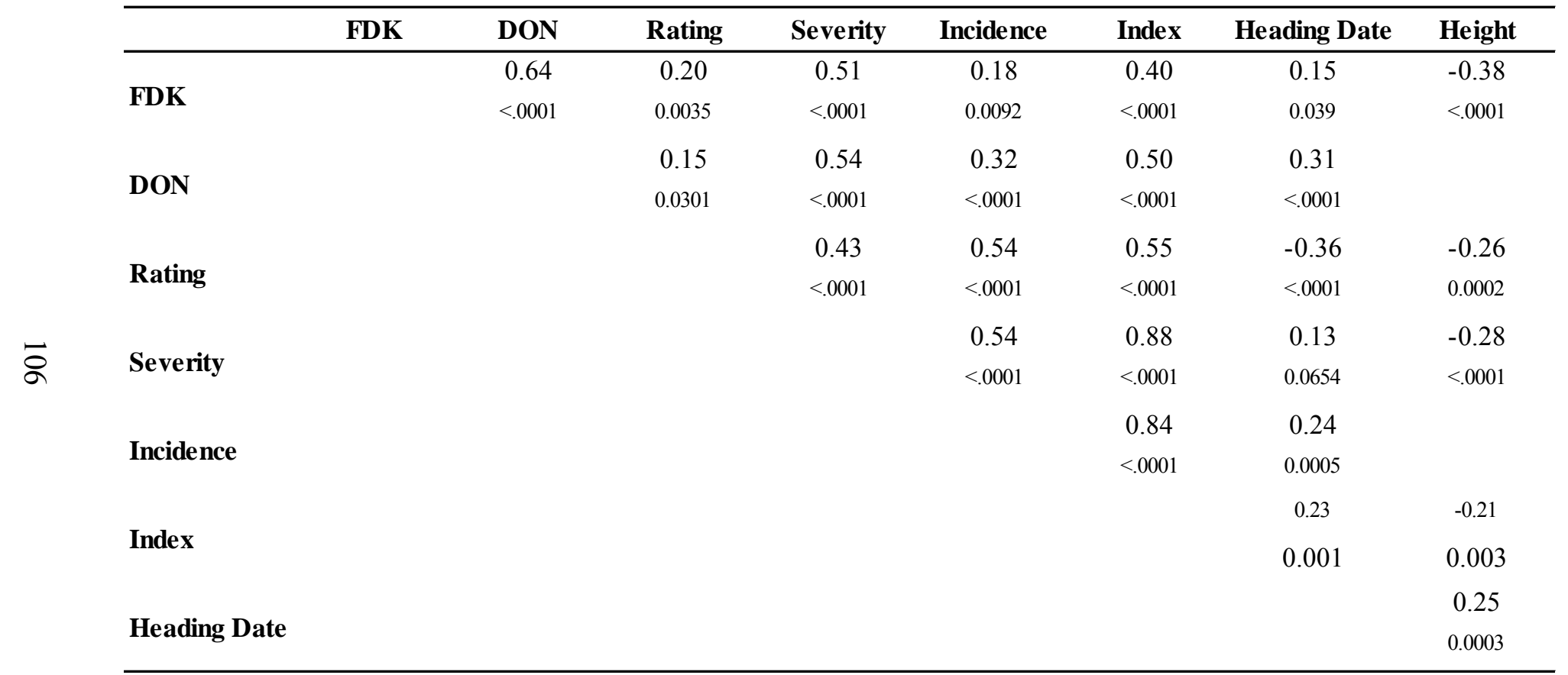

P-values are indicated below each Pearson correlation coefficient. Only significant coefficients are presented in this table. 
Table A.3.14: Mean, Maximum (Max) and Minimum (Min) yield, test weight (TWT), plant height and heading date (HD) for five populations and their different parents in Lexington 2010 and 2011, and Princeton 2011.

\begin{tabular}{|c|c|c|c|c|c|c|c|c|c|}
\hline $\mathbf{N}$ & & \multicolumn{2}{|l|}{$\begin{array}{c}\text { Yield } \\
(\mathrm{kg} / \mathrm{ha})\end{array}$} & \multicolumn{2}{|c|}{$\begin{array}{l}\text { TWT } \\
(\mathrm{kg} / \mathrm{hl})\end{array}$} & \multicolumn{2}{|c|}{$\begin{array}{c}\text { Height } \\
(\mathrm{cm})\end{array}$} & \multicolumn{2}{|l|}{$\begin{array}{c}\text { HD } \\
\text { (Julian) }\end{array}$} \\
\hline \multicolumn{10}{|c|}{ Population 1 (26R58/VA01W-476//KY97C-0574-01) } \\
\hline \multirow{3}{*}{21} & Mean & \multicolumn{2}{|l|}{4107} & \multicolumn{2}{|l|}{70.8} & \multicolumn{2}{|l|}{86.5} & \multicolumn{2}{|l|}{126.1} \\
\hline & Max & \multicolumn{2}{|l|}{5249} & \multicolumn{2}{|l|}{75.8} & \multicolumn{2}{|l|}{111.8} & \multicolumn{2}{|l|}{141.0} \\
\hline & Min & \multicolumn{2}{|l|}{2114} & \multicolumn{2}{|l|}{63.0} & \multicolumn{2}{|l|}{68.6} & \multicolumn{2}{|l|}{119.0} \\
\hline \multicolumn{10}{|c|}{ Population 2 (25R54/VA01W-476//KY97C-0574-01) } \\
\hline & Mean & \multicolumn{2}{|c|}{4095} & \multicolumn{2}{|c|}{71.9} & \multicolumn{2}{|l|}{89.9} & \multicolumn{2}{|l|}{127.0} \\
\hline \multirow[t]{2}{*}{24} & Max & \multicolumn{2}{|l|}{5419} & \multicolumn{2}{|l|}{76.1} & \multicolumn{2}{|l|}{116.8} & \multicolumn{2}{|l|}{145.0} \\
\hline & Min & 2237 & & \multicolumn{2}{|l|}{62.1} & 66.0 & & 119.0 & \\
\hline & & Populat & on $3(25 I$ & $4 / V A 011$ & $-476 / / K$ & $C-0554$ & & & \\
\hline & Mean & 4205 & & 71.1 & & 83.0 & & 126.5 & \\
\hline 36 & Max & 5471 & & 75.8 & & 101.6 & & 157.0 & \\
\hline & Min & 1758 & & 58.8 & & 66.0 & & 119.0 & \\
\hline & & & Populati & $4(25 R 7$ & $8 / V A 011$ & & & & \\
\hline & Mean & 3824 & & 70.7 & & 79.8 & & 125.2 & \\
\hline 45 & Max & 5219 & & 76.8 & & 104.1 & & 150.0 & \\
\hline & Min & 1598 & & 57.1 & & 50.8 & & 119.0 & \\
\hline & & Рори & ation 5 (1 & $93 C-12$ & $8-17-11$ & $1 W-476$ & & & \\
\hline & Mean & 4028 & & 72.6 & & 86.7 & & 125.3 & \\
\hline 29 & Max & 5328 & & 77.9 & & 119.4 & & 139.0 & \\
\hline & Min & 2584 & & 65.3 & & 61.0 & & 119.0 & \\
\hline & $25 \mathrm{R} 54$ & 4058 & (117.6) & 68.5 & $(0.4)$ & 83.8 & (1.2) & 125.8 & $(0.9)$ \\
\hline & $25 \mathrm{R} 78$ & 4350 & (170.8) & 69.8 & $(0.6)$ & 79.6 & (1.9) & 125.1 & (1.4) \\
\hline & $26 \mathrm{R} 58$ & 4499 & (170.8) & 66.3 & $(0.6)$ & 81.0 & (1.9) & 127.4 & (1.4) \\
\hline & KY93C-1238-17-1 & 4528 & (170.8) & 70.0 & $(0.6)$ & 86.4 & (1.9) & 125.3 & (1.4) \\
\hline & KY97C-0554-02 & 4881 & (170.8) & 71.0 & $(0.6)$ & 82.1 & (1.9) & 126.7 & (1.4) \\
\hline & KY97C-0574-01 & 4450 & (120.8) & 71.1 & $(0.5)$ & 85.5 & (1.4) & 127.8 & (1.0) \\
\hline & VA01W-476 & 3103 & $(85.4)$ & 73.0 & $(0.3)$ & 75.7 & $(0.9)$ & 123.5 & $(0.7)$ \\
\hline
\end{tabular}

Numbers in parentheses are standard errors (SE) 
Table A.3.15: Mean, Maximum (Max) and Minimum (Min) yield, test weight (TWT), plant height and heading date (HD) for five populations and their different parents in Lexington 2010.

\begin{tabular}{|c|c|c|c|c|c|c|c|c|c|}
\hline $\mathbf{N}$ & & $\begin{array}{c}\text { Yield } \\
(\mathrm{kg} / \mathrm{ha})\end{array}$ & & $\begin{array}{l}\text { TWT } \\
(\mathrm{kg} / \mathrm{hl})\end{array}$ & & $\begin{array}{c}\text { Height } \\
(\mathrm{cm})\end{array}$ & & $\begin{array}{c}\text { HD } \\
\text { (Julian) }\end{array}$ & \\
\hline \multicolumn{10}{|c|}{ Population 1 (26R58/VA01W-476//KY97C-0574-01) } \\
\hline \multirow{3}{*}{21} & Mean & 4041 & & 72.4 & & 83.5 & & 125.9 & \\
\hline & Max & 4743 & & 75.8 & & 99.1 & & 129.0 & \\
\hline & Min & 3084 & & 67.9 & & 68.6 & & 121.0 & \\
\hline \multicolumn{10}{|c|}{ Population $2(25 R 54 / V A 01 W-476 / / K Y 97 C-0574-01)$} \\
\hline \multirow{3}{*}{24} & Mean & 4117 & & 72.2 & & 87.9 & & 126.5 & \\
\hline & Max & 4886 & & 76.1 & & 104.1 & & 132.0 & \\
\hline & Min & 2237 & & 66.4 & & 66.0 & & 123.0 & \\
\hline \multicolumn{10}{|c|}{ Population $3(25 R 54 / V A 01 W-476 / / K Y 97 C-0554-02)$} \\
\hline \multirow{3}{*}{36} & Mean & 4069 & & 71.8 & & 82.2 & & 126.8 & \\
\hline & Max & 5307 & & 75.8 & & 99.1 & & 157.0 & \\
\hline & Min & 2634 & & 65.3 & & 68.6 & & 121.0 & \\
\hline \multicolumn{10}{|c|}{ Population $4(25 R 78 / V A 01 W-476)$} \\
\hline \multirow{3}{*}{45} & Mean & 4055 & & 72.4 & & 81.0 & & 124.1 & \\
\hline & $\operatorname{Max}$ & 5219 & & 76.8 & & 101.6 & & 128.0 & \\
\hline & Min & 2661 & & 67.6 & & 68.6 & & 121.0 & \\
\hline \multicolumn{10}{|c|}{ Population 5 (KY93C-1238-17-1/VA01W-476) } \\
\hline \multirow{10}{*}{29} & Mean & 3626 & & 83.6 & & 74.6 & & 124.4 & \\
\hline & Max & 4536 & & 99.1 & & 77.9 & & 130.0 & \\
\hline & Min & 2584 & & 68.6 & & 71.0 & & 121.0 & \\
\hline & $25 \mathrm{R} 54$ & 3774 & $(140.7)$ & 69.0 & $(0.6)$ & 84.2 & $(1.1)$ & 126.9 & $\overline{(0.5)}$ \\
\hline & $25 \mathrm{R} 78$ & 5098 & $(214.8)$ & 71.8 & $(0.8)$ & 83.0 & (1.6) & 124.3 & $(0.8)$ \\
\hline & 26R58 & 4193 & (214.8) & 66.7 & $(0.8)$ & 76.2 & (1.6) & 126.7 & $(0.8)$ \\
\hline & KY93C-1238-17-1 & 4091 & $(214.8)$ & 72.7 & $(0.8)$ & 83.0 & (1.6) & 123.7 & $(0.8)$ \\
\hline & KY97C-0554-02 & 4998 & $(214.8)$ & 72.7 & $(0.8)$ & 83.0 & (1.6) & 126.7 & $(0.8)$ \\
\hline & KY97C-0574-01 & 4231 & (151.9) & 71.7 & $(0.6)$ & 86.8 & (1.6) & 129.0 & (0.6) \\
\hline & VA01W-476 & 2946 & $(107.4)$ & 74.5 & $(0.4)$ & 76.4 & $(0.8)$ & 122.8 & $(0.4)$ \\
\hline
\end{tabular}

Numbers in parentheses are standard errors (SE) 
Table A.3.16: Mean, Maximum (Max) and Minimum (Min) yield, test weight (TWT), plant height and heading date (HD) for five populations and their different parents in Lexington 2011.

\begin{tabular}{|c|c|c|c|c|c|c|c|c|c|c|c|}
\hline $\mathbf{N}$ & & $\begin{array}{c}\text { Yield } \\
(\mathrm{kg} / \mathrm{ha})\end{array}$ & & $\begin{array}{l}\text { TWT } \\
(\mathrm{kg} / \mathrm{hl})\end{array}$ & & $\begin{array}{c}\text { Height } \\
(\mathrm{cm})\end{array}$ & & $\begin{array}{c}\text { HD } \\
\text { (Julian) }\end{array}$ & & $\begin{array}{c}\text { Rating } \\
(0-9)\end{array}$ & \\
\hline \multicolumn{12}{|c|}{ Population 1 (26R58/VA01W-476//KY97C-0574-01) } \\
\hline \multirow{3}{*}{21} & Mean & 3899 & & 68.9 & & 85.0 & & 130.7 & & 3.5 & \\
\hline & Max & 5114 & & 74.1 & & 106.7 & & 141.0 & & 7.0 & \\
\hline & Min & 2114 & & 63.0 & & 68.6 & & 128.0 & & 0.0 & \\
\hline \multicolumn{12}{|c|}{ Population $2(25 R 54 / V A 01 W-476 / / K Y 97 C-0574-01)$} \\
\hline \multirow{3}{*}{24} & Mean & 3839 & & 71.4 & & 88.0 & & 131.8 & & 2.6 & \\
\hline & Max & 5085 & & 74.8 & & 109.2 & & 145.0 & & 6.0 & \\
\hline & Min & 2440 & & 62.1 & & 71.1 & & 129.0 & & 1.0 & \\
\hline \multicolumn{12}{|c|}{ Population $3(25 R 54 / V A 01 W-476 / / K Y 97 C-0554-02)$} \\
\hline \multirow{3}{*}{36} & Mean & 4059 & & 69.7 & & 81.0 & & 130.9 & & 3.6 & \\
\hline & Max & 5367 & & 74.6 & & 101.6 & & 134.0 & & 8.0 & \\
\hline & Min & 1758 & & 58.8 & & 66.0 & & 127.0 & & 0.0 & \\
\hline \multicolumn{12}{|c|}{ Population $4(25 R 78 / V A 01 W-476)$} \\
\hline \multirow{3}{*}{45} & Mean & 3492 & & 68.7 & & 75.9 & & 129.9 & & 2.1 & \\
\hline & Max & 4971 & & 74.6 & & 96.5 & & 132.0 & & 5.0 & \\
\hline & Min & 1846 & & 57.1 & & 50.8 & & 126.0 & & 0.0 & \\
\hline \multicolumn{12}{|c|}{ Population 5 (KY93C-1238-17-1/VA01W-476) } \\
\hline \multirow{10}{*}{29} & Mean & 4075 & & 70.3 & & 82.1 & & 130.1 & & 3.2 & \\
\hline & Max & 5328 & & 74.9 & & 104.1 & & 139.0 & & 7.0 & \\
\hline & Min & 3031 & & 65.3 & & 61.0 & & 126.0 & & 1.0 & \\
\hline & $25 \mathrm{R} 54$ & 4054 & $(162.2)$ & 67.2 & $(0.6)$ & 80.9 & $(1.8)$ & 131.2 & $(0.4)$ & 5.0 & $(0.4)$ \\
\hline & $25 \mathrm{R} 78$ & 3372 & $(229.3)$ & 66.6 & $(0.8)$ & 72.8 & (2.6) & 131.0 & $(0.5)$ & 3.0 & $(0.6)$ \\
\hline & $26 \mathrm{R} 58$ & 4284 & $(229.3)$ & 63.9 & $(0.8)$ & 80.4 & (2.6) & 131.3 & $(0.5)$ & 5.7 & $(0.6)$ \\
\hline & KY93C-1238-17-1 & 4504 & (229.3) & 67.0 & $(0.8)$ & 82.1 & (2.6) & 130.3 & $(0.5)$ & 6.7 & (0.6) \\
\hline & KY97C-0554-02 & 4789 & (229.3) & 70.1 & $(0.8)$ & 83.0 & (2.6) & 131.0 & $(0.5)$ & 3.7 & $(0.6)$ \\
\hline & KY97C-0574-01 & 4317 & $(162.2)$ & 69.6 & (0.6) & 82.6 & (1.8) & 131.7 & $(0.4)$ & 4.2 & (0.4) \\
\hline & VA01W-476 & 2881 & (114.7) & 71.6 & $(0.4)$ & 70.1 & (1.3) & 127.5 & $(0.3)$ & 2.4 & $(0.3)$ \\
\hline
\end{tabular}

Numbers in parentheses are standard errors (SE) 
Table A.3.17: Mean, Maximum (Max) and Minimum (Min) yield, test weight (TWT), plant height and heading date (HD) for five populations and their different parents in Princeton 2011.

\begin{tabular}{|c|c|c|c|c|c|c|c|c|c|c|c|}
\hline $\mathbf{N}$ & & \multicolumn{2}{|l|}{$\begin{array}{c}\text { Yield } \\
(\mathrm{kg} / \mathrm{ha})\end{array}$} & \multicolumn{2}{|l|}{$\begin{array}{c}\text { TWT } \\
(\mathrm{kg} / \mathrm{hl})\end{array}$} & \multicolumn{2}{|l|}{$\begin{array}{c}\text { Height } \\
\text { (cm) }\end{array}$} & \multicolumn{2}{|l|}{$\begin{array}{c}\text { HD } \\
\text { (Julian) }\end{array}$} & \multicolumn{2}{|l|}{$\begin{array}{c}\text { Rating } \\
(0-9)\end{array}$} \\
\hline \multicolumn{12}{|c|}{ Population 1 (26R58/VA01 W-476//KY97C-0574-01) } \\
\hline \multirow{3}{*}{21} & Mean & 4380 & & 71.1 & & 91.1 & & 121.7 & & 2.5 & \\
\hline & Max & 5249 & & 74.3 & & 111.8 & & 130.0 & & 6.0 & \\
\hline & Min & 2263 & & 66.6 & & 76.2 & & 119.0 & & 0.0 & \\
\hline \multicolumn{12}{|c|}{ Population $2(25 R 54 / V A 01 W-476 / / K Y 97 C-0574-01)$} \\
\hline \multirow{3}{*}{24} & Mean & 4339 & & 72.2 & & 93.7 & & 123.2 & & 2.6 & \\
\hline & Max & 5419 & & 76.0 & & 116.8 & & 130.0 & & 5.0 & \\
\hline & Min & 2966 & & 63.3 & & 76.2 & & 119.0 & & 0.0 & \\
\hline \multicolumn{12}{|c|}{ Population 3 (25R54/VA01 W-476//KY97C-0554-02) } \\
\hline \multirow{3}{*}{36} & Mean & 4486 & & 71.7 & & 85.8 & & 121.9 & & 3.6 & \\
\hline & Max & 5471 & & 75.0 & & 101.6 & & 131.0 & & 6.0 & \\
\hline & Min & 3044 & & 67.0 & & 71.1 & & 119.0 & & 0.0 & \\
\hline \multicolumn{12}{|c|}{ Population 4 (25R78/VA01W-476) } \\
\hline \multirow{3}{*}{45} & Mean & 3927 & & 70.9 & & 82.5 & & 121.8 & & 0.9 & \\
\hline & Max & 5003 & & 75.5 & & 104.1 & & 150.0 & & 5.0 & \\
\hline & Min & 1598 & & 60.8 & & 63.5 & & 119.0 & & 0.0 & \\
\hline \multicolumn{12}{|c|}{ Population 5 (KY93C-1238-17-1/VA01 W-476) } \\
\hline \multirow{10}{*}{29} & Mean & 4383 & & 72.8 & & 94.6 & & 121.5 & & 1.3 & \\
\hline & $\operatorname{Max}$ & 5157 & & 76.4 & & 119.4 & & 130.0 & & 5.0 & \\
\hline & Min & 3326 & & 65.6 & & 71.1 & & 119.0 & & 0.0 & \\
\hline & 25R54 & 4392 & $(189.5)$ & 68.8 & $(0.5)$ & 86.4 & $(2.0)$ & 122.0 & $(0.6)$ & 6.2 & $(0.5)$ \\
\hline & $25 \mathrm{R} 78$ & 4579 & $(268.0)$ & 70.8 & $(0.6)$ & 83.0 & $(2.8)$ & 120.0 & $(0.8)$ & 5.0 & $(0.6)$ \\
\hline & $26 \mathrm{R} 58$ & 5020 & $(268.0)$ & 68.2 & $(0.6)$ & 86.4 & $(2.8)$ & 124.3 & $(0.8)$ & 6.0 & $(0.6)$ \\
\hline & KY93C-1238-17-1 & 4991 & $(268.0)$ & 70.4 & $(0.6)$ & 94.0 & $(2.8)$ & 122.0 & $(0.8)$ & 6.3 & $(0.6)$ \\
\hline & KY97C-0554-02 & 4856 & $(268.0)$ & 70.2 & $(0.6)$ & 80.4 & $(2.8)$ & 122.3 & $(0.8)$ & 6.0 & $(0.6)$ \\
\hline & KY97C-0574-01 & 4800 & $(189.5)$ & 72.1 & $(0.5)$ & 87.2 & $(2.0)$ & 122.8 & $(0.5)$ & 5.2 & $(0.5)$ \\
\hline & VA01W-476 & 3482 & $(134.0)$ & 73.1 & $(0.3)$ & 80.6 & $(1.4)$ & 120.2 & $(0.4)$ & 3.5 & $(0.3)$ \\
\hline
\end{tabular}

Numbers in parentheses are standard errors (SE) 
Table A.3.18: Mean yield, test weight (TWT), plant height and heading date (HD) for wheat lines homozygous for resistant (R) and susceptible (S) alleles at Fhbl, Lexington 2010 and 2011, and Princeton 2011.

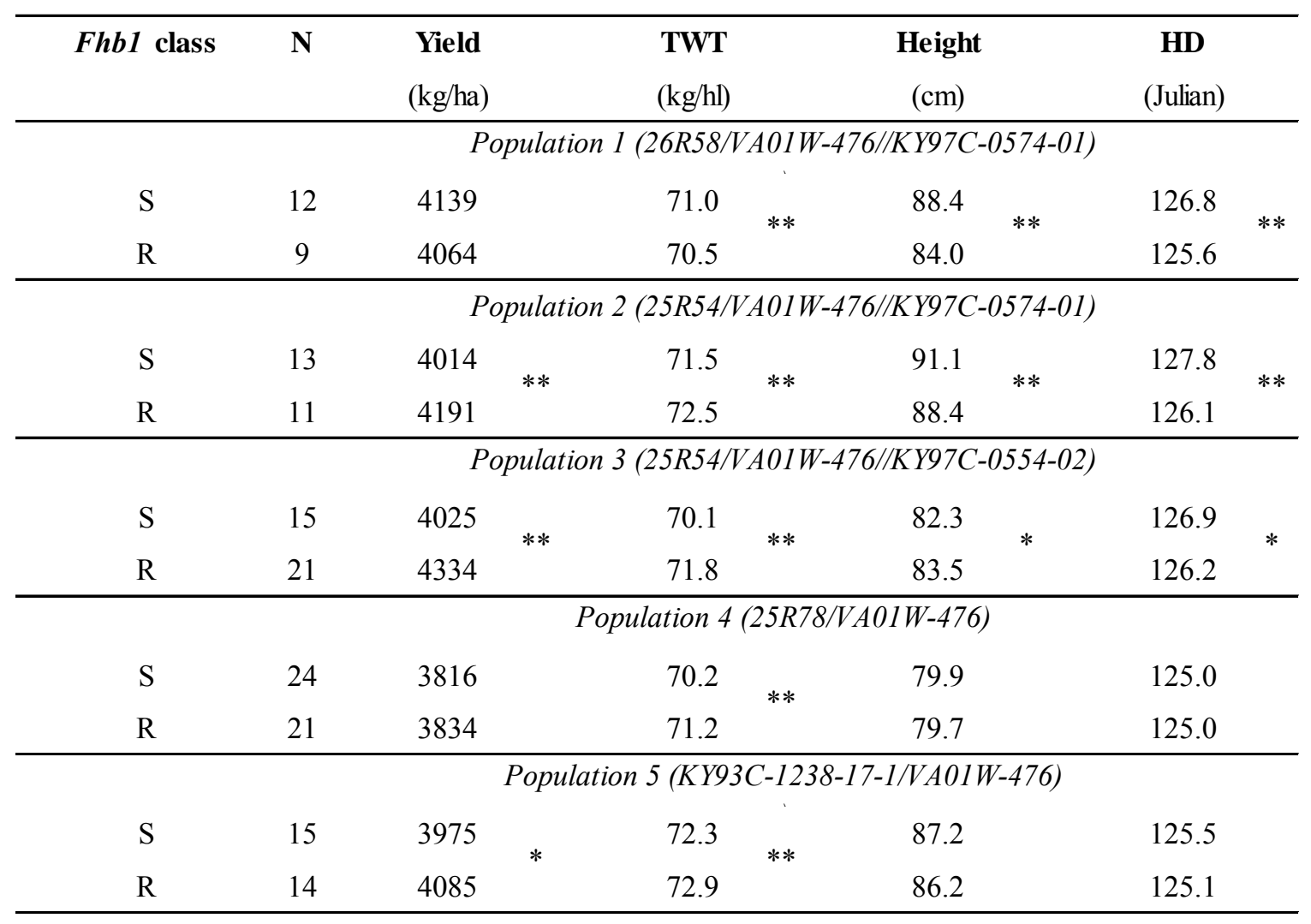

3
4 $\quad * *$ (Difference between $\mathrm{S}$ and $\mathrm{R}$ classes significant at $\mathrm{P}<0.05,0.01$, respectively) 
5 Table A.3.19: Mean yield, test weight (TWT), plant height and heading date (HD) for wheat lines homozygous for resistant (R) and susceptible (S) alleles at $F h b 1$, Lexington 2010.

\begin{tabular}{|c|c|c|c|c|c|c|}
\hline Fhb1 class & $\mathbf{N}$ & $\begin{array}{c}\text { Yield } \\
(\mathrm{kg} / \mathrm{ha})\end{array}$ & $\begin{array}{l}\text { TWT } \\
(\mathrm{kg} / \mathrm{hl})\end{array}$ & $\begin{array}{r}\text { Height } \\
\text { (cm) }\end{array}$ & $\begin{array}{c}\text { HD } \\
\text { (Julian) }\end{array}$ & \\
\hline & \multicolumn{6}{|c|}{ Population 1 (26R58/VA01W-476//KY97C-0574-01) } \\
\hline \multirow{3}{*}{$\mathrm{R}$} & 12 & 4073 & 72.6 & 85.5 & 125.6 & \multirow{2}{*}{$* *$} \\
\hline & 9 & 3999 & 72.1 & 80.7 & 126.3 & \\
\hline & \multicolumn{6}{|c|}{ Population $2(25 R 54 / V A 01 \mathrm{~W}-476 / / K Y 97 C-0574-01)$} \\
\hline $\mathrm{S}$ & 13 & 4106 & 71.7 & 88.6 & 126.9 & \multirow{2}{*}{$* *$} \\
\hline $\mathrm{R}$ & 11 & 4130 & 72.8 & 87.1 & 126.0 & \\
\hline & \multicolumn{6}{|c|}{ Population $3(25 R 54 / V A 01 \mathrm{~W}-476 / / K Y 97 C-0554-02)$} \\
\hline $\mathrm{S}$ & 15 & 3932 & 71.3 & 80.7 & \multirow{2}{*}{\multicolumn{2}{|c|}{$\begin{array}{l}126.7 \\
126.8\end{array}$}} \\
\hline \multirow[t]{2}{*}{$\mathrm{R}$} & 21 & 4167 & 72.2 & 83.3 & & \\
\hline & \multicolumn{6}{|c|}{ Population $4(25 R 78 / V A 01 W-476)$} \\
\hline $\mathrm{S}$ & 24 & 4228 & 72.2 & 81.1 & \multicolumn{2}{|l|}{124.0} \\
\hline \multirow[t]{2}{*}{$\mathrm{R}$} & 21 & 3971 & 72.6 & 80.8 & 124.2 & \\
\hline & \multicolumn{6}{|c|}{ Population 5 (KY93C-1238-17-1/VA01W-476) } \\
\hline $\mathrm{S}$ & 15 & 3666 & 74.7 & 86.4 & 124.3 & \\
\hline $\mathrm{R}$ & 14 & 3584 & 74.6 & 82.9 & 124.5 & \\
\hline
\end{tabular}

7

*, ** (Differences between $\mathrm{S}$ and $\mathrm{R}$ classes significant at $\mathrm{P}<0.05,0.01$, respectively) 
Table A.3.20: Mean yield, test weight (TWT), plant height, heading date (HD) and FHB rating measured in plots for wheat lines homozygous for resistant (R) and susceptible (S) alleles at Fhb1, Lexington 2011.

\begin{tabular}{|c|c|c|c|c|c|c|c|c|}
\hline Fhb1 class & $\mathbf{N}$ & $\begin{array}{c}\text { Yield } \\
(\mathrm{kg} / \mathrm{ha})\end{array}$ & $\begin{array}{c}\text { TWT } \\
(\mathrm{kg} / \mathrm{hl})\end{array}$ & $\begin{array}{c}\text { Height } \\
(\mathrm{cm})\end{array}$ & $\begin{array}{c}\text { HD } \\
\text { (Julian) }\end{array}$ & & $\begin{array}{c}\text { Rating } \\
(0-9)\end{array}$ & \\
\hline & \multicolumn{8}{|c|}{ Population 1 (26R58/VA01W-476//KY97C-0574-01) } \\
\hline $\mathrm{S}$ & 12 & 3940 & 69.2 & 88.2 & 130.2 & \multirow{2}{*}{$* *$} & 3.9 & \multirow{2}{*}{$* *$} \\
\hline $\mathrm{R}$ & 9 & 3845 & 69.6 & 80.8 & 131.4 & & 2.9 & \\
\hline
\end{tabular}

Population 2 (25R54/VA01W-476//KY97C-0574-01)

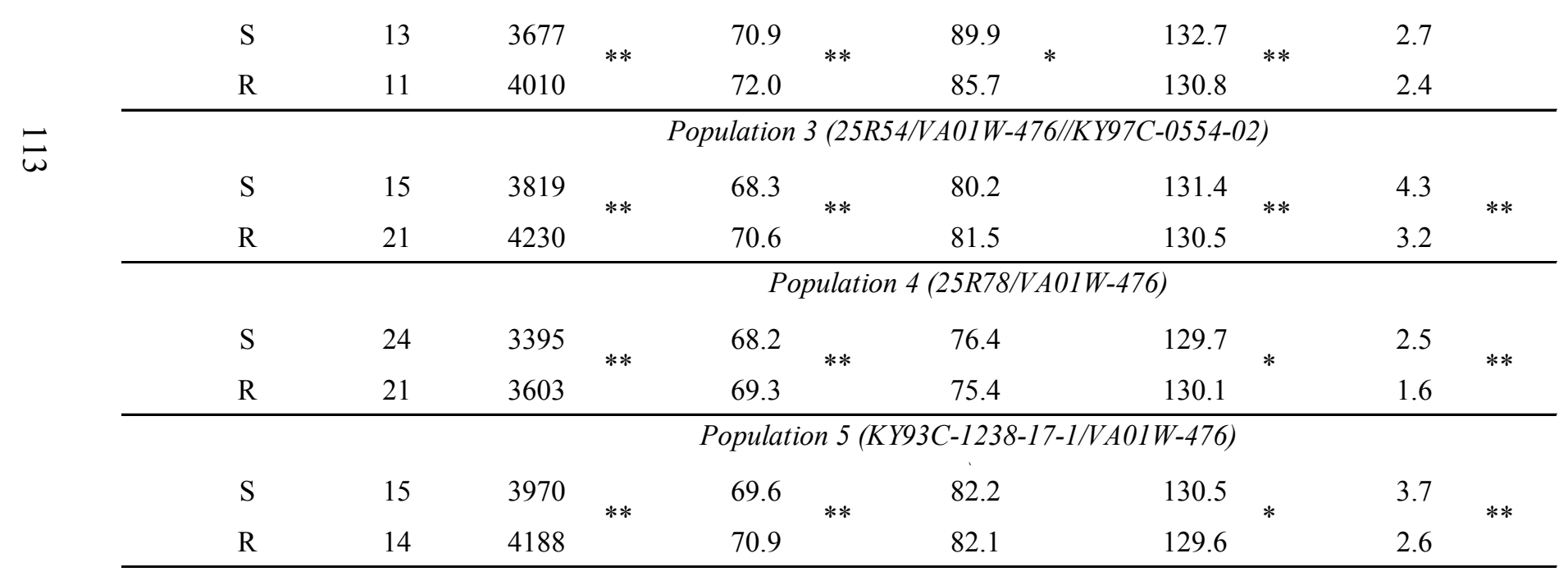

*, ** (Differences between $\mathrm{S}$ and $\mathrm{R}$ classes significant at $\mathrm{P}<0.05,0.01$, respectively) 
Table A.3.21: Mean yield, test weight (TWT), plant height, heading date (HD) and FHB rating measured in plots for wheat lines homozygous for resistant (R) and susceptible (S) alleles at Fhb1, Princeton 2011.

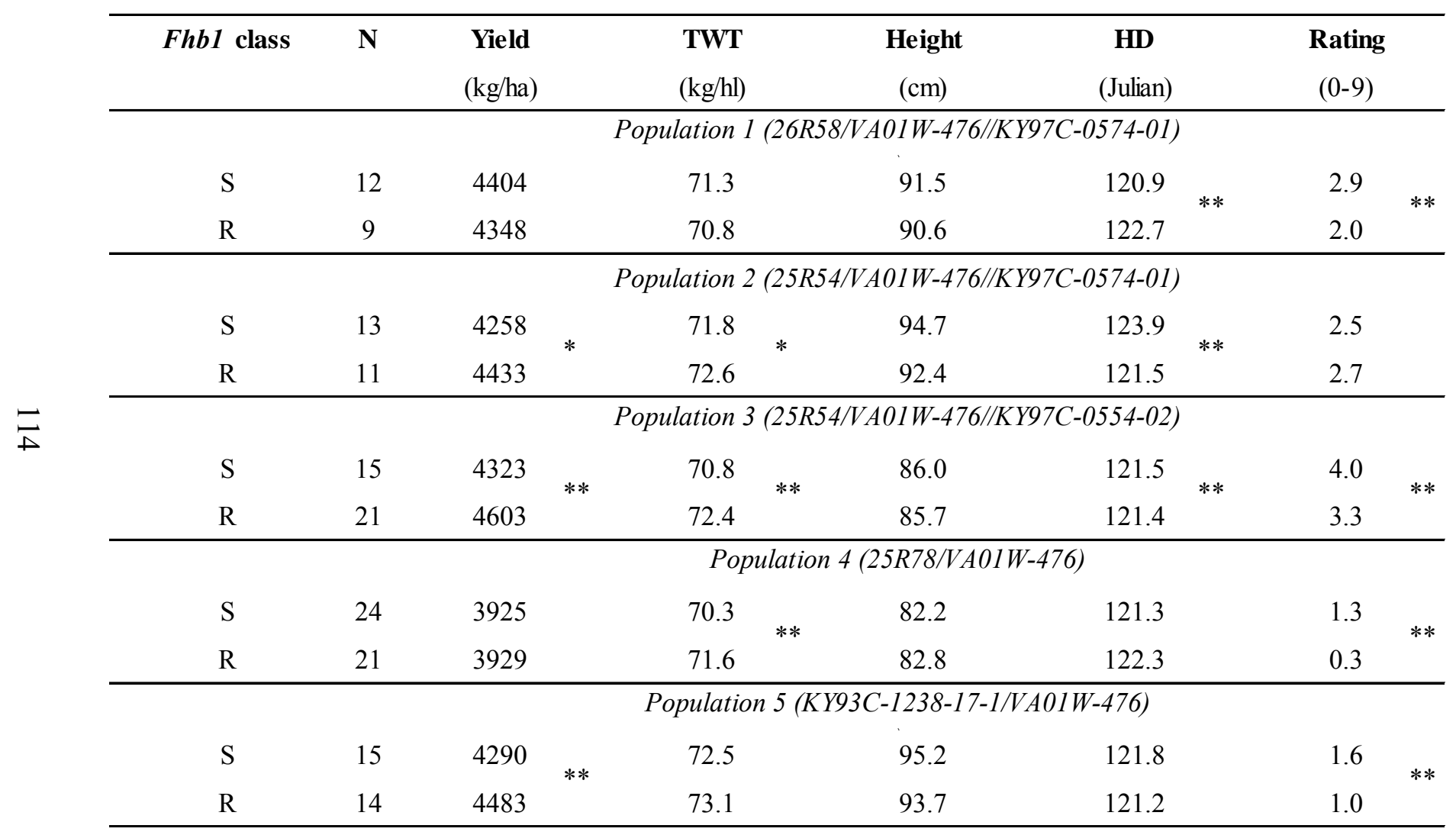

$*, * *$ (Differences between $\mathrm{S}$ and $\mathrm{R}$ classes significant at $\mathrm{P}<0.05,0.01$, respectively) 
Table A.3.22: Mean yield, test weight (TWT), plant height and heading date (HD) for homozygous susceptible $(\mathrm{S})$, resistant $(\mathrm{R})$ and heterozygous $(\mathrm{H})$ wheat lines at QFhs.nau-2DL, Lexington 2010 and 2011, and Princeton 2011.

\begin{tabular}{|c|c|c|c|c|c|c|c|c|c|}
\hline $2 D L$ class & $\mathbf{N}$ & $\begin{array}{c}\text { Yield } \\
(\mathrm{kg} / \mathrm{ha})\end{array}$ & & $\begin{array}{l}\text { TWT } \\
(\mathrm{kg} / \mathrm{hl})\end{array}$ & & $\begin{array}{c}\text { Height } \\
\text { (cm) }\end{array}$ & & $\begin{array}{c}\text { HD } \\
\text { (Julian) }\end{array}$ & \\
\hline \multicolumn{10}{|c|}{ Population 1 (26R58/VA01W-476//KY97C-0574-01) } \\
\hline $\mathrm{S}$ & 9 & 4059 & a & 70.0 & a & 84.3 & a & 126.4 & $a b$ \\
\hline $\mathrm{H}$ & 8 & 4193 & $\mathrm{~b}$ & 71.5 & $\mathrm{~b}$ & 87.2 & $\mathrm{~b}$ & 126.0 & a \\
\hline $\mathrm{R}$ & 2 & 3971 & a & 71.4 & b & 88.9 & $\mathrm{~b}$ & 126.9 & $\mathrm{~b}$ \\
\hline \multicolumn{10}{|c|}{ Population $2(25 R 54 / V A 01 W-476 / / K Y 97 C-0574-01)$} \\
\hline $\mathrm{S}$ & 4 & 4060 & a & 70.8 & a & 87.0 & a & 127.2 & $\mathrm{~b}$ \\
\hline $\mathrm{H}$ & 15 & 4071 & a & 72.0 & $\mathrm{~b}$ & 90.9 & $\mathrm{~b}$ & 127.5 & $\mathrm{~b}$ \\
\hline $\mathrm{R}$ & 4 & 4154 & a & 72.5 & $\mathrm{~b}$ & 88.0 & a & 125.0 & a \\
\hline $\mathrm{S}$ & 17 & 4314 & $\mathrm{~b}$ & 71.7 & $\mathrm{~b}$ & 83.9 & $\mathrm{~b}$ & 126.1 & a \\
\hline $\mathrm{H}$ & 9 & 4085 & a & 70.3 & $\mathrm{a}$ & 81.1 & a & 126.7 & $\mathrm{~b}$ \\
\hline $\mathrm{R}$ & 7 & 4071 & a & 70.7 & a & 83.1 & $\mathrm{~b}$ & 126.9 & $\mathrm{~b}$ \\
\hline \multicolumn{10}{|c|}{ Population $4(25 R 78 / V A 01 W-476)$} \\
\hline $\mathrm{S}$ & 12 & 3756 & a & 70.1 & a & 77.5 & a & 125.3 & $\mathrm{a}$ \\
\hline $\mathrm{H}$ & 20 & 3868 & $\mathrm{~b}$ & 71.2 & $\mathrm{~b}$ & 79.7 & $\mathrm{~b}$ & 125.0 & $\mathrm{a}$ \\
\hline $\mathrm{R}$ & 7 & 3903 & $\mathrm{~b}$ & 71.0 & $\mathrm{~b}$ & 84.6 & $\mathrm{c}$ & 125.4 & $\mathrm{a}$ \\
\hline \multicolumn{10}{|c|}{ Population 5 (KY93C-1238-17-1/VA01W-476) } \\
\hline $\mathrm{S}$ & 6 & 4025 & a & 73.0 & $\mathrm{~b}$ & 87.6 & $\mathrm{~b}$ & 125.6 & $\mathrm{~b}$ \\
\hline $\mathrm{H}$ & 15 & 4053 & $\mathrm{a}$ & 72.6 & $\mathrm{~b}$ & 87.5 & $\mathrm{~b}$ & 125.5 & $\mathrm{~b}$ \\
\hline $\mathrm{R}$ & 8 & 3984 & a & 72.1 & $\mathrm{a}$ & 84.7 & $\mathrm{a}$ & 124.7 & $\mathrm{a}$ \\
\hline
\end{tabular}

Means (by population) within the same column followed by different letters are significantly different at $\mathrm{P}<0.05$ 
Table A.3.23: Mean yield, test weight (TWT), plant height and heading date (HD) for homozygous susceptible $(\mathrm{S})$, resistant $(\mathrm{R})$ and heterozygous $(\mathrm{H})$ wheat lines at OFhs.nau-2DL, Lexington 2010.

\begin{tabular}{|c|c|c|c|c|c|c|c|c|c|}
\hline $2 D L$ class & $\mathbf{N}$ & $\begin{array}{l}\text { Yield } \\
(\mathrm{kg} / \mathrm{ha})\end{array}$ & & $\begin{array}{l}\text { TWT } \\
(\mathrm{kg} / \mathrm{hl})\end{array}$ & & $\begin{array}{l}\text { Height } \\
\text { (cm) }\end{array}$ & & $\begin{array}{c}\text { HD } \\
\text { (Julian) }\end{array}$ & \\
\hline & \multicolumn{9}{|c|}{ Population 1 (26R58/VA01W-476//KY97C-0574-01) } \\
\hline $\mathrm{S}$ & 9 & 3991 & a & 71.6 & a & 80.5 & a & 125.8 & a \\
\hline $\mathrm{H}$ & 8 & 4052 & a & 73.0 & $\mathrm{~b}$ & 85.1 & $\mathrm{~b}$ & 126.4 & $\mathrm{~b}$ \\
\hline \multirow[t]{2}{*}{$\mathrm{R}$} & 2 & 4042 & a & 73.4 & $\mathrm{~b}$ & 82.6 & $\mathrm{ab}$ & 126.0 & $\mathrm{ab}$ \\
\hline & \multicolumn{9}{|c|}{ Population $2(25 R 54 / V A 01 W-476 / / K Y 97 C-0574-01)$} \\
\hline $\mathrm{S}$ & 4 & 4182 & a & 71.6 & a & 85.1 & a & 127.2 & $\mathrm{~b}$ \\
\hline $\mathrm{H}$ & 15 & 4079 & a & 72.3 & $\mathrm{~b}$ & 88.6 & $\mathrm{~b}$ & 127.0 & $\mathrm{~b}$ \\
\hline \multirow[t]{2}{*}{$\mathrm{R}$} & 4 & 4161 & a & 72.5 & $\mathrm{~b}$ & 86.8 & $\mathrm{ab}$ & 124.3 & a \\
\hline & \multicolumn{9}{|c|}{ Population 3 (25R54/VA01W-476//KY97C-0554-02) } \\
\hline $\mathrm{S}$ & 17 & 4176 & $\mathrm{~b}$ & 72.4 & $\mathrm{~b}$ & 83.8 & $\mathrm{c}$ & 126.5 & a \\
\hline $\mathrm{H}$ & 9 & 3943 & a & 71.1 & a & 79.3 & a & 126.8 & a \\
\hline \multirow[t]{2}{*}{$\mathrm{R}$} & 7 & 3964 & $\mathrm{a}$ & 71.7 & a & 82.0 & $\mathrm{~b}$ & 126.7 & a \\
\hline & \multicolumn{9}{|c|}{ Population 4 (25R78/VA01W-476) } \\
\hline $\mathrm{S}$ & 12 & 4062 & a & 71.7 & $\mathrm{~b}$ & 79.2 & a & 123.6 & a \\
\hline $\mathrm{H}$ & 20 & 4011 & a & 72.8 & a & 80.9 & a & 123.9 & a \\
\hline \multirow[t]{2}{*}{$\mathrm{R}$} & 7 & 4248 & $\mathrm{~b}$ & 72.4 & a & 84.5 & $\mathrm{~b}$ & 124.4 & $\mathrm{~b}$ \\
\hline & \multicolumn{9}{|c|}{ Population 5 (KY93C-1238-17-1/VA01W-476) } \\
\hline $\mathrm{S}$ & 6 & 3555 & a & 75.0 & b' & 83.4 & $\mathrm{ab}$ & 124.5 & a \\
\hline $\mathrm{H}$ & 15 & 3775 & $\mathrm{~b}$ & 74.8 & $\mathrm{~b}$ & 84.6 & $\mathrm{~b}$ & 124.4 & a \\
\hline $\mathrm{R}$ & 8 & 3400 & $\mathrm{a}$ & 74.0 & a & 82.1 & a & 124.1 & a \\
\hline
\end{tabular}

Means (by population) within the same column followed by different letters are significantly different at $\mathrm{P}<0.05$ 
Table A.3.24: Mean yield, test weight (TWT), plant height, heading date (HD) and FHB rating measured in plots for homozygous susceptible (S), resistant (R) and heterozygous (H) wheat lines at QFhs.nau-2DL, Lexington 2011.

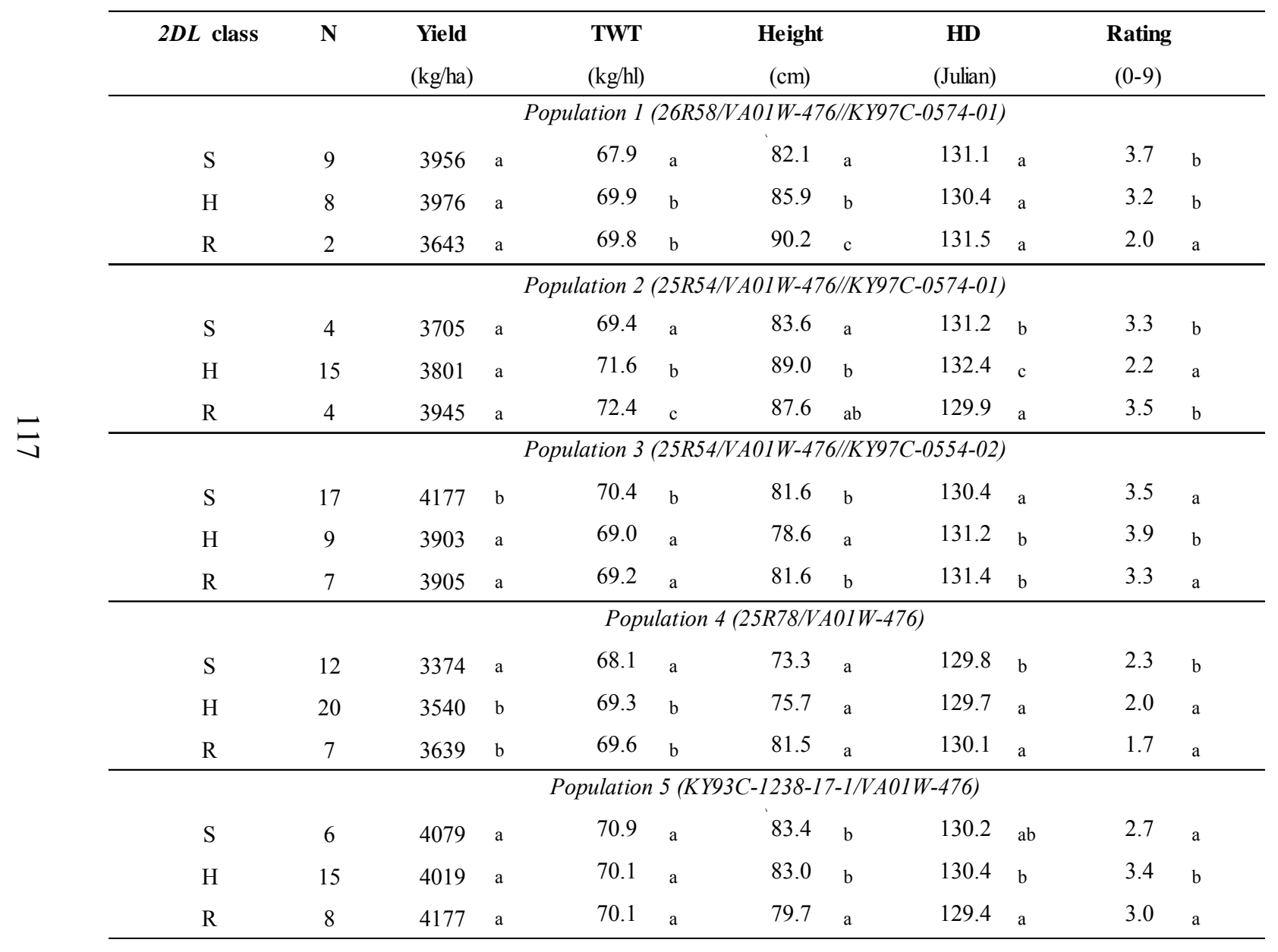

Means (by population) within the same column followed by different letters are significantly different at $\mathrm{P}<0.05$ 
Table A.3.25: Mean yield, test weight (TWT), plant height, heading date (HD) and FHB rating measured in plots for homozygous susceptible (S), resistant $(\mathrm{R})$ and heterozygous $(\mathrm{H})$ wheat lines at QFhs.nau-2DL, Princeton 2011.

\begin{tabular}{|c|c|c|c|c|c|c|c|c|c|c|c|}
\hline $2 D L$ class & $\mathbf{N}$ & $\begin{array}{c}\text { Yield } \\
(\mathrm{kg} / \mathrm{ha})\end{array}$ & & $\begin{array}{l}\text { TWT } \\
(\mathrm{kg} / \mathrm{hl})\end{array}$ & & $\begin{array}{l}\text { Height } \\
(\mathrm{cm})\end{array}$ & & $\begin{array}{c}\text { HD } \\
\text { (Julian) }\end{array}$ & & $\begin{array}{c}\text { Rating } \\
(0-9)\end{array}$ & \\
\hline & \multicolumn{11}{|c|}{ Population 1 (26R58/VA01 W-476//KY97C-0574-01) } \\
\hline $\mathrm{S}$ & 9 & 4230 & a & 70.5 & a & 90.3 & a & 122.2 & $\mathrm{~b}$ & 2.7 & a \\
\hline $\mathrm{H}$ & 8 & 4552 & $\mathrm{~b}$ & 71.5 & $\mathrm{~b}$ & 90.6 & a & 121.1 & a & 2.3 & a \\
\hline $\mathrm{R}$ & 2 & 4227 & $\mathrm{ab}$ & 71.0 & $\mathrm{ab}$ & 94.0 & a & 123.2 & $\mathrm{~b}$ & 2.0 & a \\
\hline & \multicolumn{11}{|c|}{ Population $2(25 R 54 / V A 01 W-476 / / K Y 97 C-0574-01)$} \\
\hline $\mathrm{S}$ & 4 & 4293 & a & 71.3 & a & 92.3 & $\mathrm{ab}$ & 123.2 & $\mathrm{~b}$ & 2.7 & $\mathrm{ab}$ \\
\hline $\mathrm{H}$ & 15 & 4333 & a & 72.3 & $\mathrm{~b}$ & 95.1 & $\mathrm{~b}$ & 123.3 & $\mathrm{~b}$ & 2.3 & a \\
\hline 二 & 4 & 4356 & a & 72.5 & $\mathrm{~b}$ & 89.5 & a & 120.8 & a & 3.3 & $\mathrm{~b}$ \\
\hline & \multicolumn{11}{|c|}{ Population 3 (25R54/VA01W-476//KY97C-0554-02) } \\
\hline $\mathrm{S}$ & 17 & 4588 & $\mathrm{~b}$ & 72.4 & $\mathrm{~b}$ & 86.3 & a & 121.3 & a & 3.5 & a \\
\hline $\mathrm{H}$ & 9 & 4409 & $\mathrm{ab}$ & 71.0 & a & 85.3 & a & 122.0 & $\mathrm{ab}$ & 3.9 & $\mathrm{~b}$ \\
\hline $\mathrm{R}$ & 7 & 4344 & a & 71.3 & a & 85.6 & a & 122.6 & $\mathrm{~b}$ & 3.5 & $\mathrm{ab}$ \\
\hline & \multicolumn{11}{|c|}{ Population $4(25 R 78 / V A 01 W-476)$} \\
\hline $\mathrm{S}$ & 12 & 3832 & a & 70.4 & a & 80.2 & a & 122.4 & a & 1.1 & $\mathrm{~b}$ \\
\hline $\mathrm{H}$ & 20 & 4052 & a & 71.6 & $\mathrm{~b}$ & 82.5 & a & 121.4 & a & 0.7 & a \\
\hline $\mathrm{R}$ & 7 & 3824 & a & 71.1 & $\mathrm{ab}$ & 87.8 & $\mathrm{~b}$ & 121.7 & $\mathrm{a}$ & 0.7 & $\mathrm{a}$ \\
\hline & \multicolumn{11}{|c|}{ Population 5 (KY93C-1238-17-1/VA01W-476) } \\
\hline $\mathrm{S}$ & 6 & 4440 & $\mathrm{a}$ & 73.1 & a & 96.1 & $\mathrm{~b}$ & 122.1 & $\mathrm{~b}$ & 1.2 & $\mathrm{ab}$ \\
\hline $\mathrm{H}$ & 15 & 4366 & a & 72.9 & a & 95.0 & $\mathrm{~b}$ & 121.8 & $\mathrm{~b}$ & 1.6 & $\mathrm{~b}$ \\
\hline $\mathrm{R}$ & 8 & 4374 & $\mathrm{a}$ & 72.3 & a & 92.2 & $\mathrm{a}$ & 120.7 & a & 1.0 & $\mathrm{a}$ \\
\hline
\end{tabular}

Means (by population) within the same column followed by different letters are significantly different at $\mathrm{P}<0.05$ 
Table A.3.26: Mean yield, test weight (TWT), plant height and heading date (HD) for wheat lines in each QTL combination class when all populations were combined, Lexington 2010 and 2011, and Princeton 2011.

\begin{tabular}{cccccccccc}
\hline $\begin{array}{c}\text { Fhb1 } \text { and } 2 \boldsymbol{L} \boldsymbol{L} \\
\text { combination class }\end{array}$ & N & $\begin{array}{c}\text { Yield } \\
(\mathrm{kg} / \mathrm{ha})\end{array}$ & $\begin{array}{c}\text { TWT } \\
(\mathrm{kg} / \mathrm{h})\end{array}$ & & $\begin{array}{c}\text { Height } \\
(\mathrm{cm})\end{array}$ & \multicolumn{2}{c}{$\begin{array}{c}\text { HD } \\
(\text { Julian})\end{array}$} \\
\hline RH & 29 & 4060 & $\mathrm{~b}$ & 72.0 & $\mathrm{~d}$ & 84.5 & $\mathrm{~b}$ & 125.7 & $\mathrm{ab}$ \\
RR & 15 & 4006 & $\mathrm{ab}$ & 71.5 & $\mathrm{bc}$ & 83.0 & $\mathrm{a}$ & 125.8 & $\mathrm{ab}$ \\
$\mathrm{RS}$ & 29 & 4153 & $\mathrm{c}$ & 71.7 & $\mathrm{c}$ & 83.3 & $\mathrm{a}$ & 126.0 & $\mathrm{bc}$ \\
$\mathrm{SH}$ & 38 & 3995 & $\mathrm{~b}$ & 71.3 & $\mathrm{~b}$ & 85.5 & $\mathrm{c}$ & 126.3 & $\mathrm{c}$ \\
$\mathrm{SR}$ & 13 & 4012 & $\mathrm{~b}$ & 71.5 & $\mathrm{bc}$ & 87.4 & $\mathrm{~d}$ & 125.5 & $\mathrm{a}$ \\
$\mathrm{SS}$ & 19 & 3900 & $\mathrm{a}$ & 70.3 & $\mathrm{a}$ & 82.6 & $\mathrm{a}$ & 125.9 & $\mathrm{abc}$ \\
\hline
\end{tabular}

Means within the same column followed by different letters are significantly different at $\mathrm{P}<0.05$ 
Table A.3.27: Mean, maximum (Max) and minimum (Min) whole grain hardness (WGH), whole grain protein (WGP), flour yield (FY), softness equivalent (SEQ) and flour protein (FP) for five wheat populations and their parents, Lexington 2010 and Princeton 2011.

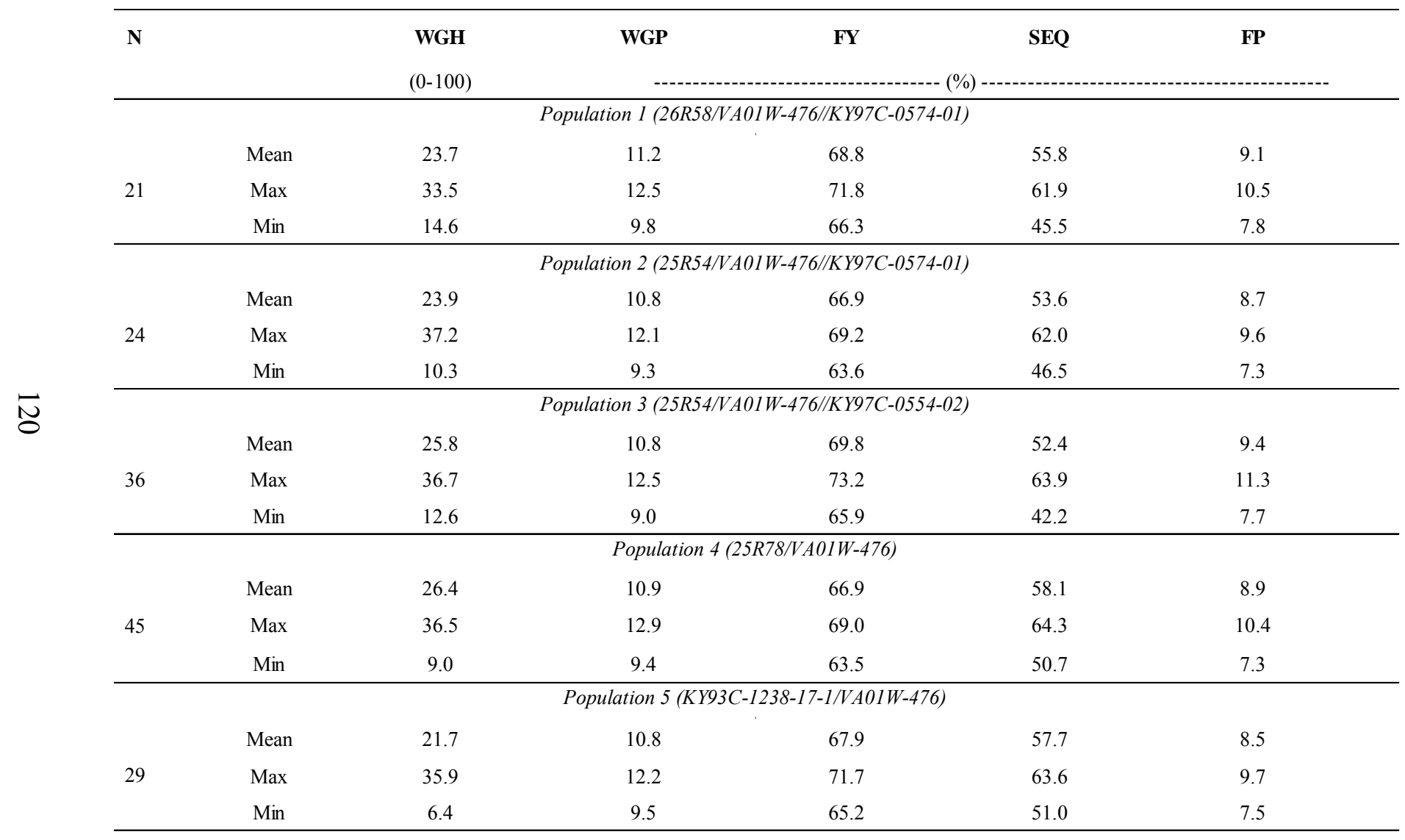


Table A.3.27 (continued): Mean, maximum (Max) and minimum (Min) whole grain hardness (WGH), whole grain protein (WGP), flour yield (FY), softness equivalent (SEQ) and flour protein (FP) for five wheat populations and their parents, Lexington 2010 and Princeton 2011.

\begin{tabular}{|c|c|c|c|c|c|c|c|c|c|c|}
\hline & \multirow{2}{*}{\multicolumn{2}{|c|}{$\begin{array}{l}\text { WGH } \\
(0-100)\end{array}$}} & \multicolumn{2}{|l|}{ WGP } & \multicolumn{2}{|l|}{$\mathbf{F Y}$} & \multicolumn{2}{|l|}{ SEQ } & \multicolumn{2}{|l|}{$\mathbf{F P}$} \\
\hline & & & & & ----- & $-\cdots($ & & & & -- \\
\hline $25 \mathrm{R} 54$ & 17.3 & (1.3) & 10.2 & $(0.1)$ & 70.1 & $(0.2)$ & 62.1 & $(0.5)$ & 8.3 & $(0.1)$ \\
\hline $25 \mathrm{R} 78$ & 24.7 & (1.9) & 10.6 & $(0.2)$ & 69.2 & $(0.3)$ & 59.1 & $(0.7)$ & 8.6 & $(0.1)$ \\
\hline 26R58 & 20.8 & (1.9) & 10.7 & $(0.2)$ & 68.4 & $(0.3)$ & 58.2 & $(0.7)$ & 8.7 & $(0.1)$ \\
\hline KY93C-1238-17-1 & 17.5 & (1.9) & 10.6 & $(0.2)$ & 70.5 & $(0.3)$ & 61.9 & $(0.7)$ & 8.3 & $(0.1)$ \\
\hline KY97C-0554-02 & 30.8 & (1.9) & 10.2 & $(0.2)$ & 72.8 & $(0.3)$ & 48.0 & $(0.7)$ & 9.3 & $(0.1)$ \\
\hline KY97C-0574-01 & 22.9 & (1.3) & 10.8 & $(0.1)$ & 69.0 & $(0.2)$ & 56.5 & $(0.5)$ & 8.7 & $(0.1)$ \\
\hline VA01W-476 & 28.9 & $(1.0)$ & 11.6 & $(0.1)$ & 64.8 & $(0.2)$ & 54.8 & $(0.4)$ & 9.5 & $(0.1)$ \\
\hline
\end{tabular}

Numbers in parentheses are standard errors (SE) 
Table A.3.28: Mean, maximum (Max) and minimum (Min) whole grain hardness (WGH), whole grain protein (WGP), flour yield (FY), softness equivalent (SEQ) and flour protein (FP) for five wheat populations and their parents, Lexington 2010.

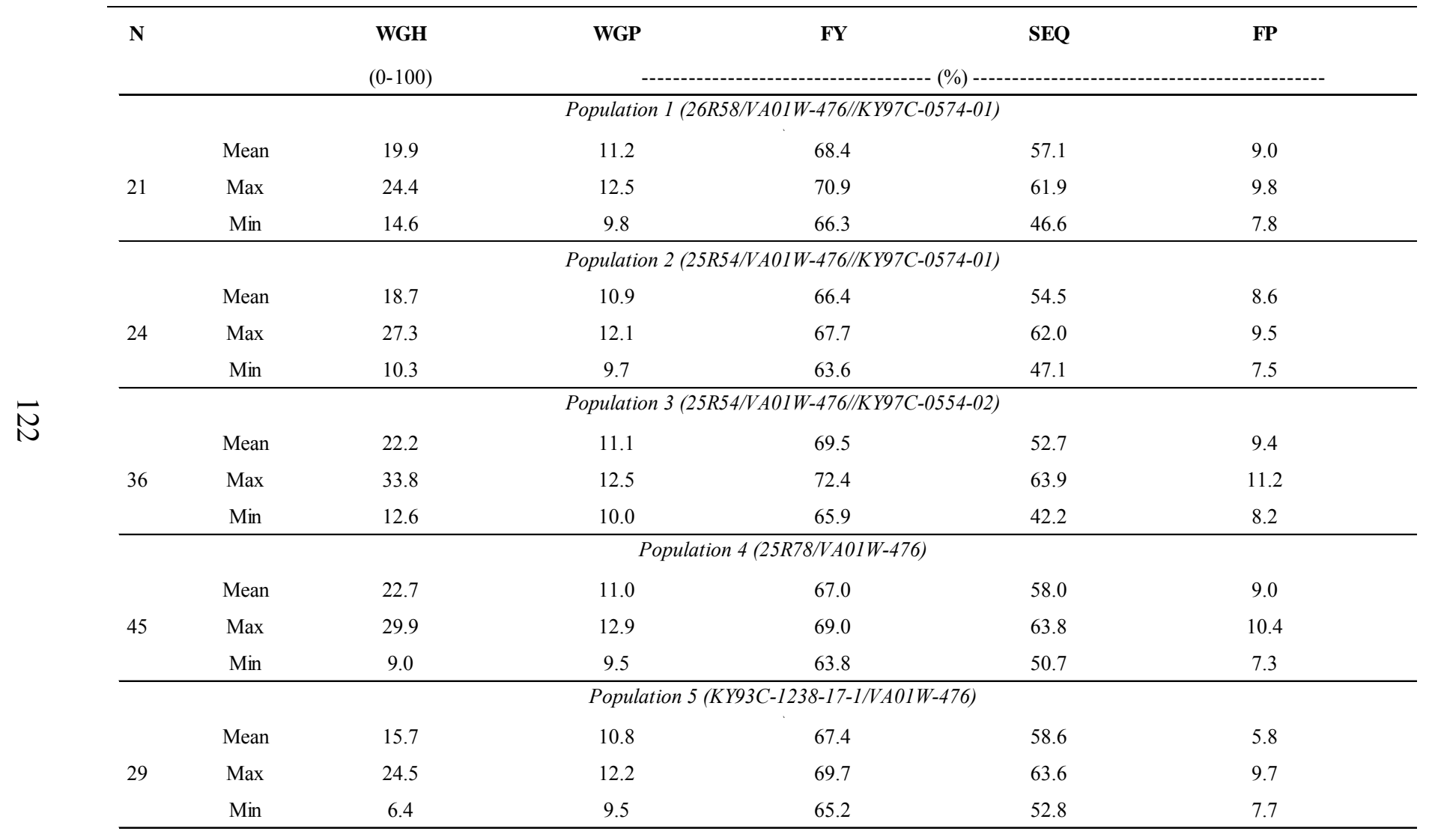


Table A.3.28 (continued): Mean, maximum (Max) and minimum (Min) whole grain hardness (WGH), whole grain protein (WGP), flour yield (FY), softness equivalent (SEQ) and flour protein (FP) for five wheat populations and their parents, Lexington 2010.

\begin{tabular}{|c|c|c|c|c|c|c|c|c|c|c|}
\hline & \multirow{2}{*}{\multicolumn{2}{|c|}{$\begin{array}{c}\text { WGH } \\
(0-100)\end{array}$}} & \multicolumn{2}{|l|}{ WGP } & \multicolumn{2}{|l|}{ FY } & \multicolumn{2}{|l|}{ SEQ } & \multicolumn{2}{|l|}{ FP } \\
\hline & & & & $---\cdot-$ & 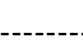 & -- $(\%)$ & 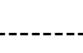 & $---\cdot-$ & 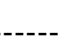 & \\
\hline $25 \mathrm{R} 54$ & 14.0 & $(0.7)$ & 10.5 & $(0.1)$ & 69.8 & $(0.2)$ & 63.5 & $(0.6)$ & 8.3 & $\overline{(0.1)}$ \\
\hline $25 \mathrm{R} 78$ & 20.0 & (1.1) & 10.6 & $(0.2)$ & 69.1 & $(0.4)$ & 58.2 & $(0.9)$ & 8.6 & $(0.2)$ \\
\hline $26 \mathrm{R} 58$ & 16.1 & (1.1) & 10.6 & $(0.2)$ & 67.5 & $(0.4)$ & 60.3 & $(0.9)$ & 8.4 & $(0.2)$ \\
\hline KY93C-1238-17-1 & 11.3 & (1.1) & 10.4 & $(0.2)$ & 70.5 & $(0.4)$ & 62.8 & $(0.9)$ & 8.2 & $(0.2)$ \\
\hline KY97C-0554-02 & 28.0 & (1.1) & 10.5 & $(0.2)$ & 72.8 & $(0.4)$ & 47.6 & $(0.9)$ & 9.4 & $(0.2)$ \\
\hline KY97C-0574-01 & 18.8 & $(0.8)$ & 10.8 & $(0.3)$ & 68.5 & $(0.3)$ & 58.0 & $(0.6)$ & 8.6 & $(0.1)$ \\
\hline VA01W-476 & 26.6 & $(0.6)$ & 11.8 & $(0.1)$ & 64.6 & $(0.2)$ & 54.4 & $(0.4)$ & 9.6 & $(0.1)$ \\
\hline
\end{tabular}

Numbers in parentheses are standard errors (SE) 
Table A.3.29: Mean, maximum (Max) and minimum (Min) whole grain hardness (WGH), whole grain protein (WGP), flour yield (FY), softness equivalent (SEQ) and flour protein (FP) for five wheat populations and their parents, Princeton 2011.

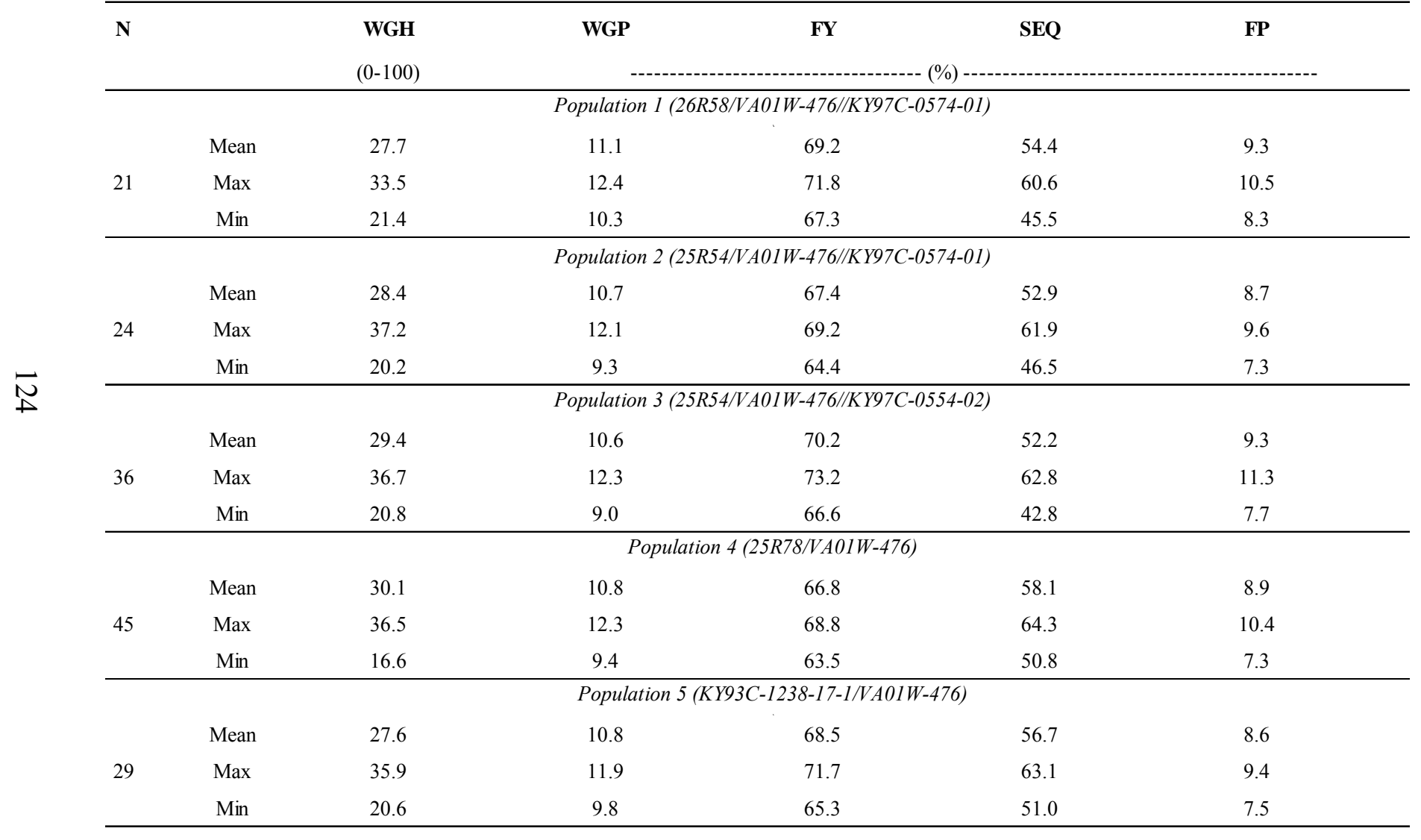


Table A.3.29 (continued): Mean, maximum (Max) and minimum (Min) whole grain hardness (WGH), whole grain protein (WGP), flour yield (FY), softness equivalent (SEQ) and flour protein (FP) for five wheat populations and their parents, Princeton 2011.

\begin{tabular}{|c|c|c|c|c|c|c|c|c|c|c|}
\hline & \multirow{2}{*}{\multicolumn{2}{|c|}{$\begin{array}{l}\text { WGH } \\
(0-100)\end{array}$}} & \multicolumn{2}{|l|}{ WGP } & \multicolumn{2}{|l|}{$\mathbf{F Y}$} & \multicolumn{2}{|l|}{ SEQ } & \multicolumn{2}{|l|}{ FP } \\
\hline & & & & ----- & & ---- (\%) (\%) & & & & \\
\hline $25 \mathrm{R} 54$ & 21.3 & $(0.9)$ & 10.0 & $(0.1)$ & 70.5 & $(0.3)$ & 60.6 & $(0.3)$ & 8.3 & $(0.1)$ \\
\hline $25 \mathrm{R} 78$ & 29.5 & (1.3) & 10.6 & $(0.2)$ & 69.2 & $(0.4)$ & 60.1 & $(0.5)$ & 8.7 & $(0.2)$ \\
\hline $26 \mathrm{R} 58$ & 25.4 & (1.3) & 10.8 & $(0.2)$ & 69.3 & $(0.4)$ & 56.1 & $(0.5)$ & 9.0 & $(0.2)$ \\
\hline KY93C-1238-17-1 & 23.7 & (1.3) & 10.7 & $(0.2)$ & 70.6 & $(0.4)$ & 60.9 & $(0.5)$ & 8.3 & $(0.2)$ \\
\hline KY97C-0554-02 & 33.6 & (1.3) & 9.9 & $(0.2)$ & 72.9 & $(0.4)$ & 48.4 & $(0.5)$ & 9.1 & $(0.2)$ \\
\hline KY97C-0574-01 & 27.0 & $(0.9)$ & 10.8 & $(0.1)$ & 69.5 & $(0.3)$ & 55.0 & $(0.3)$ & 8.9 & $(0.1)$ \\
\hline VA01W-476 & 31.2 & $(0.6)$ & 11.3 & $(0.1)$ & 64.9 & $(0.2)$ & 55.2 & $(0.2)$ & 9.4 & $(0.1)$ \\
\hline
\end{tabular}

Numbers in parentheses are standard errors (SE) 
Table A.3.30: Mean, maximum (Max) and minimum (Min) Gluten performance index (GPI), flour lactic acid solvent retention capacity (LASRC), flour sucrose solvent retention capacity (SSRC), flour water solvent retention capacity (WSRC), flour sodium carbonate solvent retention capacity (SCSRC), estimated cookie diameter (ECD), wheat meal sedimentation test (SDS) and wheat meal SCSRC (WMSCSRC) for five wheat populations and their parents in Lexington 2010 and Princeton 2011.

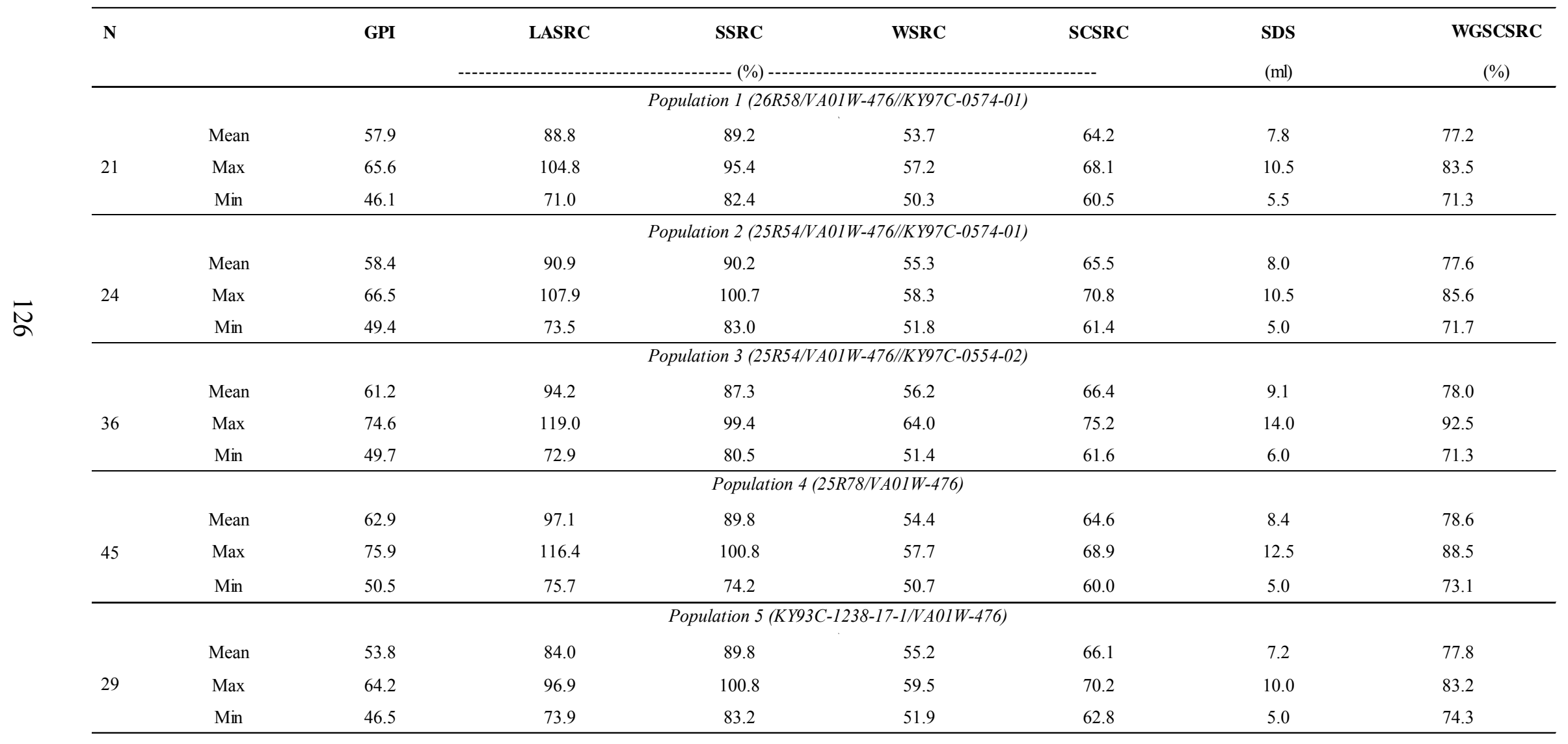


Table A.3.30 (continued): Mean, maximum (Max) and minimum (Min) Gluten performance index (GPI), flour lactic acid solvent retention capacity (LASRC), flour sucrose solvent retention capacity (SSRC), flour water solvent retention capacity (WSRC), flour sodium carbonate solvent retention capacity (SCSRC), estimated cookie diameter (ECD), wheat meal sedimentation test (SDS) and wheat meal SCSRC (WMSCSRC) for five wheat populations and their parents in Lexington 2010 and Princeton 2011.

\begin{tabular}{|c|c|c|c|c|c|c|c|c|c|c|c|c|c|c|}
\hline & \multicolumn{2}{|l|}{ GPI } & \multicolumn{2}{|c|}{ LASRC } & \multicolumn{2}{|l|}{ SSRC } & \multicolumn{2}{|l|}{ WSRC } & \multicolumn{2}{|l|}{ SCSRC } & \multicolumn{2}{|c|}{$\begin{array}{l}\text { SDS } \\
\text { (ml) }\end{array}$} & \multicolumn{2}{|c|}{$\begin{array}{c}\text { WGSCSRC } \\
(\%)\end{array}$} \\
\hline $25 \mathrm{R} 54$ & 60.7 & $(0.0)$ & 90.5 & $(1.0)$ & 87.2 & $(0.7)$ & 52.1 & $(0.4)$ & 61.9 & $(0.3)$ & 7.0 & $(0.3)$ & 76.9 & $(0.4)$ \\
\hline $25 \mathrm{R} 78$ & 58.6 & $(0.0)$ & 89.6 & $(1.5)$ & 88.2 & $(1.0)$ & 55.1 & $(0.5)$ & 64.8 & $(0.5)$ & 7.6 & $(0.4)$ & 76.8 & $(0.6)$ \\
\hline $26 \mathrm{R} 58$ & 51.0 & $(0.0)$ & 75.4 & $(1.5)$ & 84.5 & $(1.0)$ & 52.9 & $(0.5)$ & 63.3 & $(0.5)$ & 6.4 & $(0.4)$ & 76.3 & $(0.6)$ \\
\hline KY93C-1238-17-1 & 47.3 & $(0.0)$ & 71.2 & $(1.5)$ & 86.0 & (1.0) & 54.5 & $(0.5)$ & 64.7 & $(0.5)$ & 4.3 & $(0.4)$ & 77.2 & $(0.6)$ \\
\hline KY97C-0554-02 & 59.7 & $(0.0)$ & 91.1 & $(1.5)$ & 84.6 & (1.0) & 57.3 & $(0.5)$ & 68.2 & $(0.5)$ & 8.9 & $(0.4)$ & 80.2 & $(0.6)$ \\
\hline KY97C-0574-01 & 60.2 & $(0.0)$ & 93.3 & (1.1) & 90.7 & $(0.7)$ & 53.8 & $(0.4)$ & 64.4 & $(0.3)$ & 8.1 & $(0.3)$ & 76.8 & $(0.4)$ \\
\hline VA01W-476 & 65.3 & $(0.0)$ & 103.8 & $(0.8)$ & 92.6 & $(0.5)$ & 56.0 & $(0.3)$ & 66.5 & $(0.2)$ & 9.3 & $(0.3)$ & 80.5 & $(0.3)$ \\
\hline
\end{tabular}

Numbers in parentheses are standard errors (SE) 
Table A.3.31: Mean, maximum (Max) and minimum (Min) Gluten performance index (GPI), flour lactic acid solvent retention capacity (LASRC), flour sucrose solvent retention capacity (SSRC), flour water solvent retention capacity (WSRC), flour sodium carbonate solvent retention capacity (SCSRC), estimated cookie diameter (ECD), wheat meal sedimentation test (SDS) and wheat meal SCSRC (WMSCSRC) for five wheat populations and their parents in Lexington 2010.

\begin{tabular}{|c|c|c|c|c|c|c|c|c|c|}
\hline \multirow{2}{*}{$\mathbf{N}$} & & GPI & LASRC & SSRC & WSRC & SCSRC & \multirow{2}{*}{$\begin{array}{l}\text { ECD } \\
(\mathrm{cm})\end{array}$} & \multirow{2}{*}{$\begin{array}{l}\text { SDS } \\
\text { (ml) }\end{array}$} & \multirow{2}{*}{$\begin{array}{c}\text { WGSCSRC } \\
(\%)\end{array}$} \\
\hline & & & ---------.- & $---(\%)-$ & - & & & & \\
\hline & \multicolumn{9}{|c|}{ Population $1(26 R 58 / V A 01$ W-476//KY97C-0574-01) } \\
\hline & Mean & 59.0 & 91.2 & 90.5 & 52.9 & 64.1 & 17.9 & 7.9 & 77.2 \\
\hline 21 & $\operatorname{Max}$ & 65.6 & 104.8 & 95.4 & 56.9 & 68.1 & 18.4 & 10.5 & 81.6 \\
\hline & Min & 50.3 & 73.0 & 86.9 & 50.3 & 60.5 & 17.5 & 5.5 & 73.3 \\
\hline & \multicolumn{9}{|c|}{ Population $2(25 R 54 / V A 01$ W-476//KY97C-0574-01) } \\
\hline & Mean & 59.2 & 92.8 & 90.8 & 54.5 & 66.1 & 17.8 & 8.3 & 78.3 \\
\hline 24 & Max & 66.2 & 107.9 & 97.1 & 57.0 & 70.8 & 18.2 & 10.5 & 84.9 \\
\hline & Min & 51.5 & 80.8 & 83.8 & 51.8 & 62.0 & 17.3 & 5.5 & 74.1 \\
\hline & & \multicolumn{8}{|c|}{ Population 3 (25R54/VA01W-476//KY97C-0554-02) } \\
\hline & Mean & 63.4 & 96.9 & 87.0 & 55.7 & 65.8 & 17.9 & 9.9 & 77.4 \\
\hline 36 & $\operatorname{Max}$ & 73.6 & 119.0 & 97.1 & 60.7 & 73.6 & 18.5 & 14.0 & 88.7 \\
\hline & Min & 61.6 & 82.8 & 80.5 & 51.4 & 61.6 & 17.3 & 6.0 & 71.3 \\
\hline & & \multicolumn{8}{|c|}{ Population $4(25 R 78 / V A 01 W-476)$} \\
\hline & Mean & 65.1 & 98.8 & 88.3 & 53.6 & 63.7 & 18.1 & 8.9 & 77.5 \\
\hline 45 & $\operatorname{Max}$ & 75.9 & 114.2 & 95.3 & 57.2 & 67.9 & 18.9 & 12.0 & 83.3 \\
\hline & Min & 53.3 & 83.0 & 74.2 & 50.7 & 60.0 & 17.5 & 5.5 & 73.1 \\
\hline & \multicolumn{9}{|c|}{ Population 5 (KY93C-1238-17-1/VA01W-476) } \\
\hline & Mean & 55.6 & 86.0 & 89.3 & 54.8 & 65.5 & 18.0 & 7.4 & 77.5 \\
\hline 29 & $\operatorname{Max}$ & 64.2 & 96.9 & 83.3 & 58.2 & 68.8 & 18.5 & 10.0 & 81.4 \\
\hline & Min & 49.1 & 76.5 & 98.2 & 51.9 & 62.8 & 17.4 & 5.5 & 74.4 \\
\hline
\end{tabular}


Table A.3.31 (continued): Mean, maximum (Max) and minimum (Min) Gluten performance index (GPI), flour lactic acid solvent retention capacity (LASRC), flour sucrose solvent retention capacity (SSRC), flour water solvent retention capacity (WSRC), flour sodium carbonate solvent retention capacity (SCSRC), estimated cookie diameter (ECD), wheat meal sedimentation test (SDS) and wheat meal SCSRC (WMSCSRC) for five wheat populations and their parents in Lexington 2010.

\begin{tabular}{|c|c|c|c|c|c|c|c|c|c|c|c|c|c|c|c|c|}
\hline & GPI & & LASRC & & $\begin{array}{l}\text { SSRC } \\
-(\%)--\end{array}$ & & WSRC & & SCSRC & & $\begin{array}{l}\text { ECD } \\
(\mathrm{cm})\end{array}$ & & $\begin{array}{l}\text { SDS } \\
(\mathrm{ml})\end{array}$ & & $\begin{array}{c}\text { WGSCSRC } \\
(\%)\end{array}$ & \\
\hline $25 \mathrm{R} 54$ & 0.6 & $(0.0)$ & 90.4 & $(1.2)$ & 86.5 & $(0.9)$ & 51.4 & $(0.4)$ & 62.1 & $(0.3)$ & 18.4 & $(0.1)$ & 7.1 & $(0.3)$ & 77.3 & $(0.4)$ \\
\hline $25 \mathrm{R} 78$ & 0.6 & $(0.0)$ & 88.9 & (1.8) & 87.4 & (1.4) & 54.7 & $(0.7)$ & 64.3 & $(0.4)$ & 18.1 & $(0.1)$ & 7.7 & $(0.5)$ & 75.4 & $(0.7)$ \\
\hline $26 \mathrm{R} 58$ & 0.5 & $(0.0)$ & 75.2 & (1.8) & 82.7 & (1.4) & 51.7 & $(0.7)$ & 64.1 & $(0.4)$ & 18.5 & $(0.1)$ & 6.3 & $(0.5)$ & 76.2 & $(0.7)$ \\
\hline KY93C-1238-17-1 & 0.5 & $(0.0)$ & 70.3 & (1.8) & 84.1 & (1.4) & 53.9 & $(0.7)$ & 63.5 & $(0.4)$ & 18.5 & $(0.1)$ & 4.3 & $(0.5)$ & 77.2 & $(0.7)$ \\
\hline KY97C-0554-02 & 0.6 & $(0.0)$ & 94.2 & (1.8) & 84.1 & (1.4) & 56.2 & $(0.7)$ & 67.1 & $(0.4)$ & 17.8 & $(0.1)$ & 8.8 & $(0.5)$ & 78.6 & $(0.7)$ \\
\hline KY97C-0574-01 & 0.6 & $(0.0)$ & 95.4 & $(1.3)$ & 92.6 & $(1.0)$ & 52.5 & $(0.5)$ & 64.7 & $(0.3)$ & 17.8 & $(0.1)$ & 8.3 & $(0.4)$ & 77.1 & $0.5)$ \\
\hline VA01W-476 & 0.7 & $(0.0)$ & 107.3 & $(0.9)$ & 92.1 & $(0.7)$ & 55.3 & $(0.3)$ & 65.5 & $(0.2)$ & 17.7 & $(0.0)$ & 10.2 & $(0.3)$ & 80.1 & $(0.3)$ \\
\hline
\end{tabular}

Numbers in parentheses are standard errors (SE) 
Table A.3.32: Mean, maximum (Max) and minimum (Min) Gluten performance index (GPI), flour lactic acid solvent retention capacity (LASRC), flour sucrose solvent retention capacity (SSRC), flour water solvent retention capacity (WSRC), flour sodium carbonate solvent retention capacity (SCSRC), estimated cookie diameter (ECD), wheat meal sedimentation test (SDS) and wheat meal SCSRC (WMSCSRC) for five wheat populations and their parents in Princeton 2011.

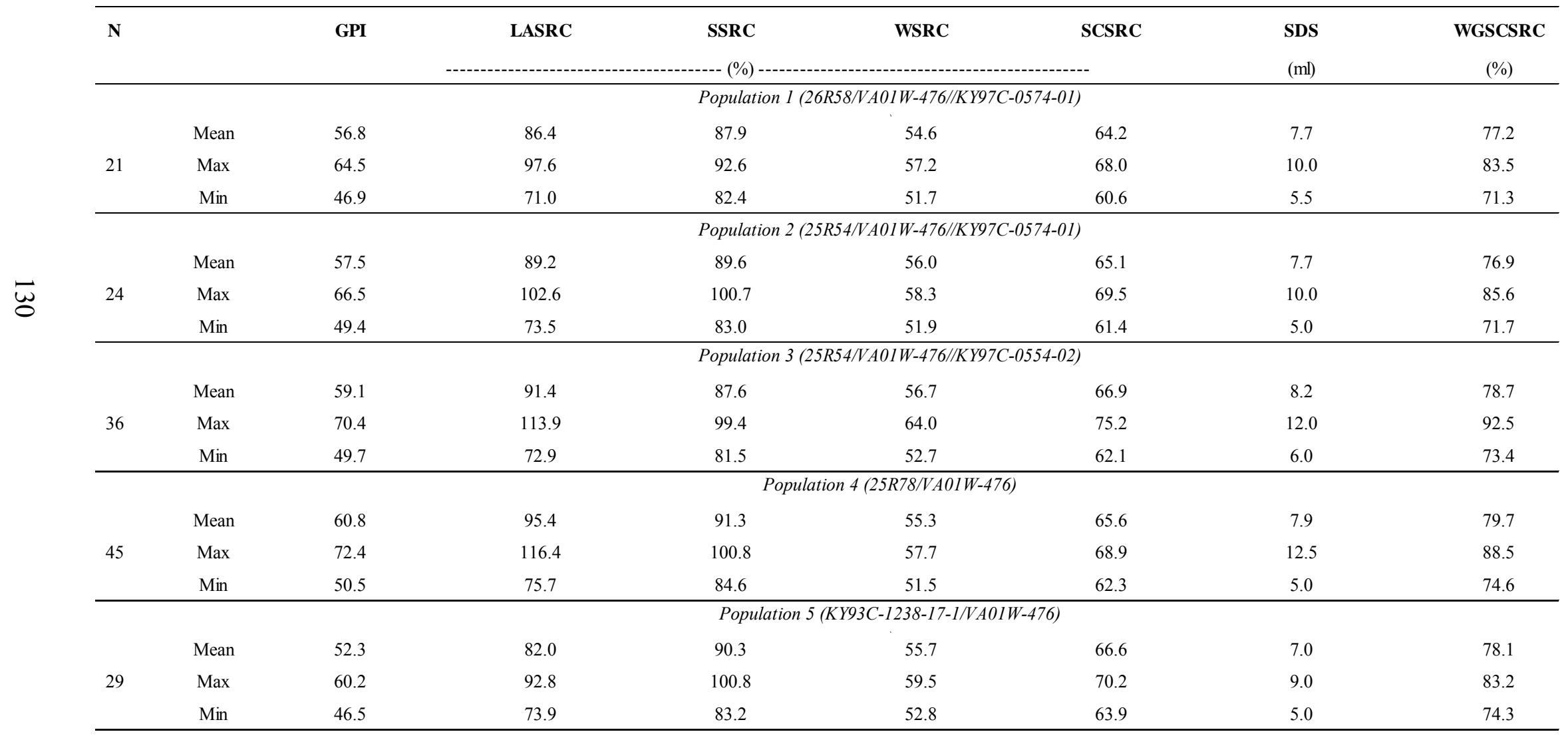


Table A.3.32: Mean, maximum (Max) and minimum (Min) Gluten performance index (GPI), flour lactic acid solvent retention capacity (LASRC), flour sucrose solvent retention capacity (SSRC), flour water solvent retention capacity (WSRC), flour sodium carbonate solvent retention capacity (SCSRC), estimated cookie diameter (ECD), wheat meal sedimentation test (SDS) and wheat meal SCSRC (WMSCSRC) for five wheat populations and their parents in Princeton 2011.

\begin{tabular}{|c|c|c|c|c|c|c|c|c|c|c|c|c|c|c|}
\hline & GPI & & LASRC & & SSRC & & WSRC & & SCSRC & & SDS & & WGSCSRC & \\
\hline $25 \mathrm{R} 54$ & 0.6 & $(0.0)$ & 90.5 & (1.1) & 88.0 & $(1.0)$ & 52.8 & $(0.3)$ & 61.6 & $(0.4)$ & 6.9 & $(0.4)$ & 76.4 & $(0.6)$ \\
\hline $25 \mathrm{R} 78$ & 0.6 & $(0.0)$ & 90.3 & (1.6) & 89.0 & (1.4) & 55.6 & $(0.5)$ & 65.2 & (0.6) & 7.5 & $(0.6)$ & 78.1 & $(0.8)$ \\
\hline 26R58 & 0.5 & $(0.0)$ & 75.5 & (1.6) & 86.2 & (1.4) & 54.0 & $(0.5)$ & 62.6 & (0.6) & 6.5 & (0.6) & 76.5 & $(0.8)$ \\
\hline KY93C-1238-17-1 & 0.5 & $(0.0)$ & 72.2 & (1.6) & 88.0 & (1.4) & 55.1 & $(0.5)$ & 65.9 & (0.6) & 4.3 & $(0.6)$ & 77.2 & $(0.8)$ \\
\hline KY97C-0554-02 & 0.6 & $(0.0)$ & 88.1 & (1.6) & 85.1 & (1.4) & 58.3 & $(0.5)$ & 69.2 & $(0.6)$ & 9.0 & $(0.6)$ & 81.8 & $(0.8)$ \\
\hline KY97C-0574-01 & 0.6 & $(0.0)$ & 91.3 & (1.1) & 88.8 & (1.0) & 55.2 & $(0.3)$ & 64.1 & $(0.4)$ & 7.9 & $(0.4)$ & 76.4 & $(0.6)$ \\
\hline VA01W-476 & 0.6 & $(0.0)$ & 100.2 & $(0.8)$ & 93.2 & $(0.7)$ & 56.7 & $(0.2)$ & 67.5 & $(0.3)$ & 8.5 & $(0.3)$ & 81.0 & $(0.4)$ \\
\hline
\end{tabular}

Numbers in parentheses are standard errors (SE) 
Table A.3.33: Mean whole grain hardness (WGH), whole grain protein (WGP), flour yield (FY), softness equivalent (SEQ) and flour protein (FP) for wheat lines homozygous for resistant (R) and susceptible (S) alleles at Fhb1, Lexington 2010 and Princeton 2011.

\begin{tabular}{|c|c|c|c|c|c|c|c|c|c|c|c|}
\hline Fhb1 class & $\mathbf{N}$ & WGH & & WGP & & $\mathbf{F Y}$ & & SEQ & & \multicolumn{2}{|l|}{ FP } \\
\hline & \multicolumn{11}{|c|}{ Population 1 (26R58/VA01W-476//KY97C-0574-01) } \\
\hline $\mathrm{S}$ & 12 & 24.0 & \multirow{2}{*}{ * } & 11.3 & \multirow{2}{*}{$* *$} & 68.9 & \multirow{2}{*}{$* *$} & 55.0 & \multirow{2}{*}{ ** } & 9.2 & \multirow{2}{*}{ ** } \\
\hline $\mathrm{R}$ & 9 & 23.4 & & 11.1 & & 68.6 & & 56.7 & & 9.0 & \\
\hline & \multicolumn{11}{|c|}{ Population $2(25 R 54 / V A 01 W-476 / / K Y 97 C-0574-01)$} \\
\hline $\mathrm{S}$ & 13 & 24.0 & & 10.9 & \multirow{2}{*}{$* *$} & 67.1 & \multirow{2}{*}{$* *$} & 53.9 & \multirow{2}{*}{$* *$} & 8.7 & \\
\hline $\mathrm{R}$ & 11 & 23.7 & & 10.8 & & 66.8 & & 53.3 & & 8.8 & \\
\hline & \multicolumn{11}{|c|}{ Population $3(25 R 54 / V A 01 W-476 / / K Y 97 C-0554-02)$} \\
\hline $\mathrm{S}$ & 15 & 26.3 & \multirow{2}{*}{$* *$} & 10.7 & \multirow{2}{*}{$* *$} & 70.1 & \multirow{2}{*}{$* *$} & 52.9 & \multirow{2}{*}{$* *$} & 9.3 & \multirow{2}{*}{ ** } \\
\hline $\mathrm{R}$ & 21 & 25.4 & & 11.0 & & 69.6 & & 52.1 & & 9.4 & \\
\hline & \multicolumn{11}{|c|}{ Population $4(25 R 78 / V A 01 W-476)$} \\
\hline $\mathrm{S}$ & 24 & 26.8 & \multirow{2}{*}{$* *$} & 10.9 & & 67.0 & \multirow{2}{*}{$* *$} & 58.4 & \multirow{2}{*}{ ** } & 8.9 & \\
\hline $\mathrm{R}$ & 21 & 26.0 & & 10.9 & & 66.7 & & 57.6 & & 8.9 & \\
\hline & \multicolumn{11}{|c|}{ Population 5 (KY93C-1238-17-1/VA01W-476) } \\
\hline $\mathrm{S}$ & 15 & 21.3 & \multirow{2}{*}{$* *$} & 10.9 & \multirow{2}{*}{ * } & 68.2 & \multirow{2}{*}{$* *$} & 58.2 & \multirow{2}{*}{ ** } & 8.6 & \multirow{2}{*}{ ** } \\
\hline $\mathrm{R}$ & 14 & 22.1 & & 10.8 & & 67.7 & & 57.1 & & 8.5 & \\
\hline
\end{tabular}

*, ** (Difference between $\mathrm{S}$ and $\mathrm{R}$ classes significant at $\mathrm{P}<0.05,0.01$, respectively) 
Table A.3.34: Mean gluten performance index (GPI), flour lactic acid solvent retention capacity (LASRC), flour sucrose solvent retention capacity (SSRC), flour water solvent retention capacity (WSRC), flour sodium carbonate solvent retention capacity (SCSRC), wheat meal sedimentation test (SDS) and wheat meal SCSRC (WMSCSRC) for wheat lines homozygous for resistant (R) and susceptible (S) alleles at Fhb1, Lexington 2010 and Princeton 2011.

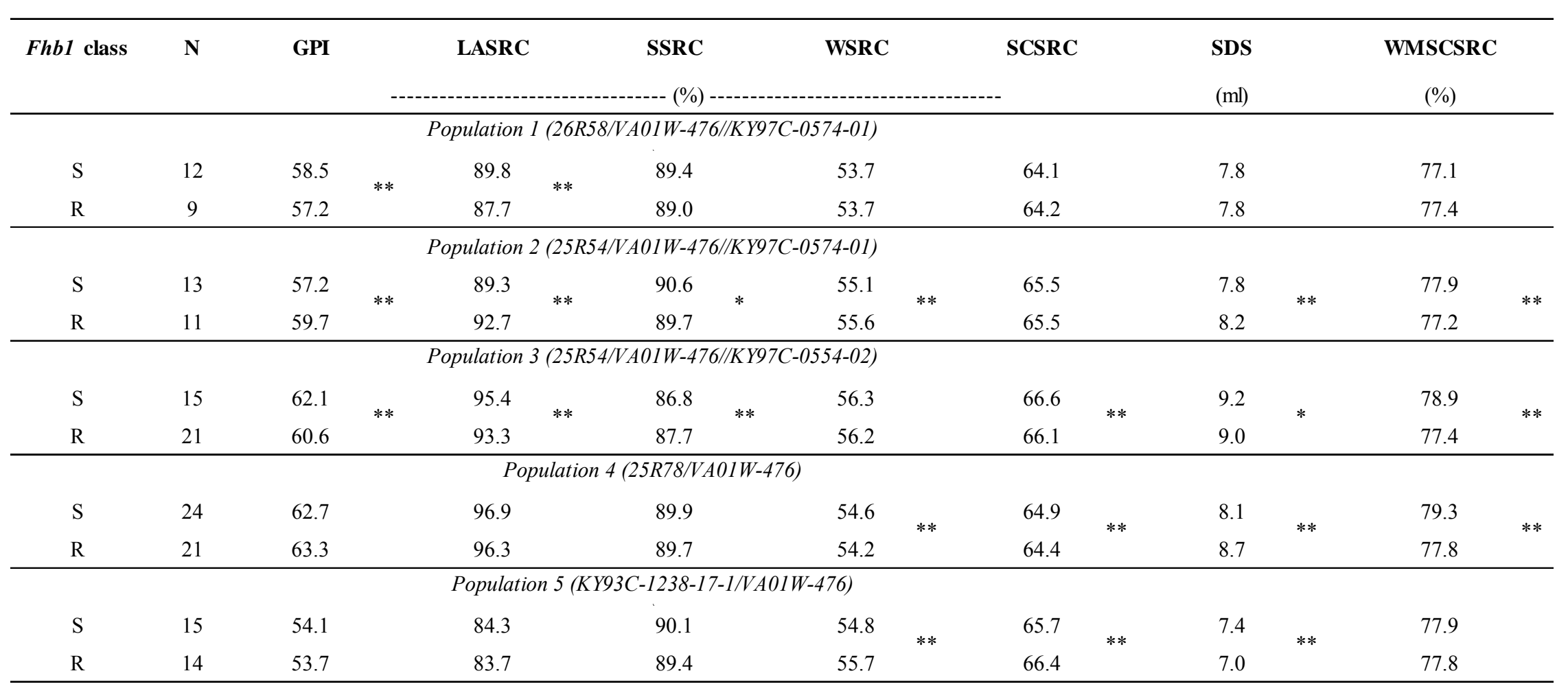

*,** (Difference between $\mathrm{S}$ and $\mathrm{R}$ classes significant at $\mathrm{P}<0.05,0.01$, respectively) 
Table A.3.35: Mean whole grain hardness (WGH), whole grain protein (WGP), flour yield (FY), softness equivalent (SEQ) and flour protein (FP) for wheat lines homozygous for resistant (R) and susceptible (S) alleles at Fhbl, Lexington 2010.

\begin{tabular}{|c|c|c|c|c|c|c|c|c|c|c|c|}
\hline Fhb1 class & $\mathbf{N}$ & WGH & & WGP & & $\mathbf{F Y}$ & & SEQ & & FP & \\
\hline & \multicolumn{11}{|c|}{ Population 1 (26R58/VA01W-476//KY97C-0574-01) } \\
\hline $\mathrm{S}$ & 12 & 20.3 & \multirow{2}{*}{ ** } & 11.3 & \multirow{2}{*}{$* *$} & 68.5 & \multirow{2}{*}{$* *$} & 56.4 & \multirow{2}{*}{$* *$} & 9.0 & \\
\hline $\mathrm{R}$ & 9 & 19.4 & & 11.1 & & 68.2 & & 58.0 & & 8.9 & \\
\hline & \multicolumn{11}{|c|}{ Population 2 (25R54/VA01W-476//KY97C-0574-01) } \\
\hline $\mathrm{S}$ & 13 & 18.5 & & 11.0 & \multirow{2}{*}{ ** } & \multicolumn{2}{|c|}{66.4} & \multicolumn{2}{|l|}{54.6} & \multicolumn{2}{|l|}{8.7} \\
\hline $\mathrm{R}$ & 11 & 19.0 & & 10.9 & & \multicolumn{2}{|l|}{66.4} & \multicolumn{2}{|l|}{54.3} & \multicolumn{2}{|l|}{8.6} \\
\hline & \multicolumn{11}{|c|}{ Population 3 (25R54/VA01W-476//KY97C-0554-02) } \\
\hline $\mathrm{S}$ & 15 & 22.9 & \multirow{2}{*}{$* *$} & 11.0 & \multirow{2}{*}{$* *$} & 69.8 & \multirow{2}{*}{$* *$} & 53.1 & \multirow{2}{*}{$* *$} & 9.3 & \multirow{2}{*}{$* *$} \\
\hline $\mathrm{R}$ & 21 & 21.6 & & 11.3 & & 69.2 & & 52.4 & & 9.5 & \\
\hline & \multicolumn{11}{|c|}{ Population $4(25 R 78 / V A 01 W-476)$} \\
\hline $\mathrm{S}$ & 24 & 23.1 & \multirow{2}{*}{$* *$} & 11.0 & & 67.2 & \multirow{2}{*}{$* *$} & 58.3 & \multirow{2}{*}{$* *$} & 8.9 & \\
\hline $\mathrm{R}$ & 21 & 22.3 & & 11.0 & & 66.7 & & 57.7 & & 9.0 & \\
\hline & \multicolumn{11}{|c|}{ Population 5 (KY93C-1238-17-1/VA01W-476) } \\
\hline $\mathrm{S}$ & 15 & 15.3 & \multirow{2}{*}{$* *$} & 10.9 & \multirow{2}{*}{$* *$} & 67.7 & \multirow{2}{*}{ ** } & 58.9 & \multirow{2}{*}{$*$} & 8.6 & \multirow{2}{*}{$* *$} \\
\hline $\mathrm{R}$ & 14 & 16.1 & & 10.7 & & 67.1 & & 58.3 & & 8.4 & \\
\hline
\end{tabular}

*, ** (Differences between $\mathrm{S}$ and $\mathrm{R}$ classes significant at $\mathrm{P}<0.05,0.01$, respectively) 
Table A.3.36: Mean gluten performance index (GPI), flour lactic acid solvent retention capacity (LASRC), flour sucrose solvent retention capacity (SSRC), flour water solvent retention capacity (WSRC), flour sodium carbonate solvent retention capacity (SCSRC), estimated cookie diameter (ECD), wheat meal sedimentation test (SDS) and wheat meal SCSRC (WMSCSRC) for wheat lines homozygous for resistant (R) and susceptible (S) alleles at Fhb1, Lexington 2010.

\begin{tabular}{|c|c|c|c|c|c|c|c|c|c|c|c|c|c|c|c|c|c|}
\hline Fhb1 class & $\mathbf{N}$ & GPI & & LASRC & & $\begin{array}{l}\text { SSRC } \\
--(\%)\end{array}$ & & WSRC & --- & SCSRC & & $\begin{array}{l}\text { ECD } \\
(\mathrm{cm})\end{array}$ & & $\begin{array}{l}\text { SDS } \\
\text { (ml) }\end{array}$ & & $\begin{array}{c}\text { WMSCSRC } \\
(\%)\end{array}$ & \\
\hline $\mathrm{S}$ & 12 & 59.5 & \multirow{2}{*}{$* *$} & 92.1 & \multirow{2}{*}{$* *$} & \multirow{2}{*}{\multicolumn{2}{|c|}{$\begin{array}{l}90.8 \\
90.1\end{array}$}} & \multirow{2}{*}{\multicolumn{2}{|c|}{$\begin{array}{l}52.8 \\
53.0\end{array}$}} & \multirow{2}{*}{\multicolumn{2}{|c|}{$\begin{array}{l}64.1 \\
64.2\end{array}$}} & 18.0 & \multirow{2}{*}{$* *$} & 8.0 & \multicolumn{3}{|c|}{77.1} \\
\hline $\mathrm{R}$ & 9 & 58.3 & & 89.9 & & & & & & & & 17.9 & & 7.8 & \multicolumn{3}{|c|}{77.5} \\
\hline $\mathrm{S}$ & 13 & 58.2 & \multirow{2}{*}{$* *$} & 91.6 & \multirow{2}{*}{$* *$} & \multirow{2}{*}{\multicolumn{2}{|c|}{$\begin{array}{l}91.3 \\
90.3\end{array}$}} & \multirow{2}{*}{\multicolumn{2}{|c|}{$\begin{array}{l}54.3 \\
54.8\end{array}$}} & \multirow{2}{*}{\multicolumn{2}{|c|}{$\begin{array}{l}66.2 \\
65.9\end{array}$}} & \multirow{2}{*}{\multicolumn{2}{|c|}{$\begin{array}{l}17.7 \\
17.8\end{array}$}} & 8.1 & \multirow{2}{*}{$* *$} & \multirow{2}{*}{$\begin{array}{l}78.4 \\
78.1\end{array}$} & \\
\hline $\mathrm{R}$ & 11 & 60.3 & & 94.2 & & & & & & & & & & 8.5 & & & \\
\hline $\mathrm{S}$ & 15 & 64.5 & \multirow{2}{*}{$* *$} & 98.3 & \multirow{2}{*}{$* *$} & 86.1 & \multirow{2}{*}{$* *$} & 55.9 & & 66.0 & \multirow{2}{*}{$* *$} & 17.9 & \multirow{2}{*}{$* *$} & 10.0 & & 78.4 & \multirow{2}{*}{$* *$} \\
\hline $\mathrm{R}$ & 21 & 62.6 & & 98.9 & & 87.7 & & 55.6 & & 65.6 & & 17.8 & & 9.8 & & 76.6 & \\
\hline $\mathrm{S}$ & 24 & 64.9 & & 98.5 & & 88.1 & & 53.8 & \multirow{2}{*}{$* *$} & 63.9 & \multirow{2}{*}{$* *$} & 18.1 & \multirow{2}{*}{$*$} & 8.7 & \multirow{2}{*}{ * } & 78.2 & \multirow{2}{*}{$* *$} \\
\hline $\mathrm{R}$ & 21 & 65.3 & & 99.2 & & 88.6 & & 53.4 & & 63.5 & & 18.0 & & 9.1 & & 76.8 & \\
\hline $\mathrm{S}$ & 15 & 56.1 & \multirow{2}{*}{ * } & 86.5 & & 89.2 & & 54.3 & \multirow{2}{*}{$* *$} & 65.1 & \multirow{2}{*}{$* *$} & 18.1 & & 7.6 & \multirow{2}{*}{$* *$} & 75.5 & \multirow{2}{*}{$* *$} \\
\hline $\mathrm{R}$ & 14 & 55.1 & & 85.5 & & 89.4 & & 55.4 & & 65.9 & & 18.0 & & 7.1 & & 75.5 & \\
\hline
\end{tabular}

*, ** (Difference between $\mathrm{S}$ and $\mathrm{R}$ classes significant at $\mathrm{P}<0.05,0.01$, respectively) 
Table A.3.37: Mean whole grain hardness (WGH), whole grain protein (WGP), flour yield (FY), softness equivalent (SEQ) and flour protein (FP) for wheat lines homozygous for resistant (R) and susceptible (S) alleles at Fhb1, Princeton 2011.

\begin{tabular}{|c|c|c|c|c|c|c|c|c|c|c|}
\hline Fhb1 class & $\mathbf{N}$ & WGH & & WGP & $\mathbf{F Y}$ & & \multicolumn{2}{|l|}{ SEQ } & & FP \\
\hline & \multicolumn{10}{|c|}{ Population 1 (26R58/VA01W-476//KY97C-0574-01) } \\
\hline $\mathrm{S}$ & 12 & 28.0 & & 11.2 & 69.3 & \multirow{2}{*}{ * } & 53.6 & \multirow{2}{*}{$* *$} & 9.3 & \multirow{2}{*}{$* *$} \\
\hline $\mathrm{R}$ & 9 & 27.3 & & 11.0 & 69.0 & & 55.5 & & 9.1 & \\
\hline & \multicolumn{10}{|c|}{ Population $2(25 R 54 / V A 01 W-476 / / K Y 97 C-0574-01)$} \\
\hline $\mathrm{S}$ & 13 & 28.7 & & 10.7 & 67.2 & \multirow{2}{*}{$* *$} & 53.4 & \multirow{2}{*}{$* *$} & 8.7 & \\
\hline $\mathrm{R}$ & 11 & 28.1 & & 10.6 & 67.7 & & 52.4 & & 8.7 & \\
\hline & \multicolumn{10}{|c|}{ Population 3 (25R54/VA01W-476//KY97C-0554-02) } \\
\hline $\mathrm{S}$ & 15 & 29.7 & \multirow{2}{*}{$*$} & 10.4 & 70.5 & \multirow{2}{*}{$* *$} & 52.7 & \multirow{2}{*}{$* *$} & 9.2 & \\
\hline $\mathrm{R}$ & 21 & 29.1 & & 10.7 & 70.0 & & 51.8 & & 9.3 & \\
\hline & \multicolumn{10}{|c|}{ Population $4(25 R 78 / V A 01 W-476)$} \\
\hline $\mathrm{S}$ & 24 & 30.4 & & 10.8 & 66.8 & & 58.6 & \multirow{2}{*}{$* *$} & 8.9 & \\
\hline $\mathrm{R}$ & 21 & 29.8 & & 10.8 & 66.8 & & 57.6 & & 8.9 & \\
\hline & \multicolumn{10}{|c|}{ Population 5 (KY93C-1238-17-1/VA01W-476) } \\
\hline $\mathrm{S}$ & 15 & 27.2 & \multirow[t]{2}{*}{ * } & 10.8 & 68.7 & \multirow{2}{*}{$* *$} & 57.4 & \multirow{2}{*}{$* *$} & 8.7 & \\
\hline $\mathrm{R}$ & 14 & 28.0 & & 10.8 & 68.3 & & 55.9 & & 8.6 & \\
\hline
\end{tabular}

*,** (Differences between $\mathrm{S}$ and $\mathrm{R}$ classes significant at $\mathrm{P}<0.05,0.01$, respectively) 
Table A.3.38: Mean gluten performance index (GPI), flour lactic acid solvent retention capacity (LASRC), flour sucrose solvent retention capacity (SSRC), flour water solvent retention capacity (WSRC), flour sodium carbonate solvent retention capacity (SCSRC), wheat meal sedimentation test (SDS) and wheat meal SCSRC (WMSCSRC) for wheat lines homozygous for resistant (R) and susceptible (S) alleles at $F h b 1$. Princeton 2011.

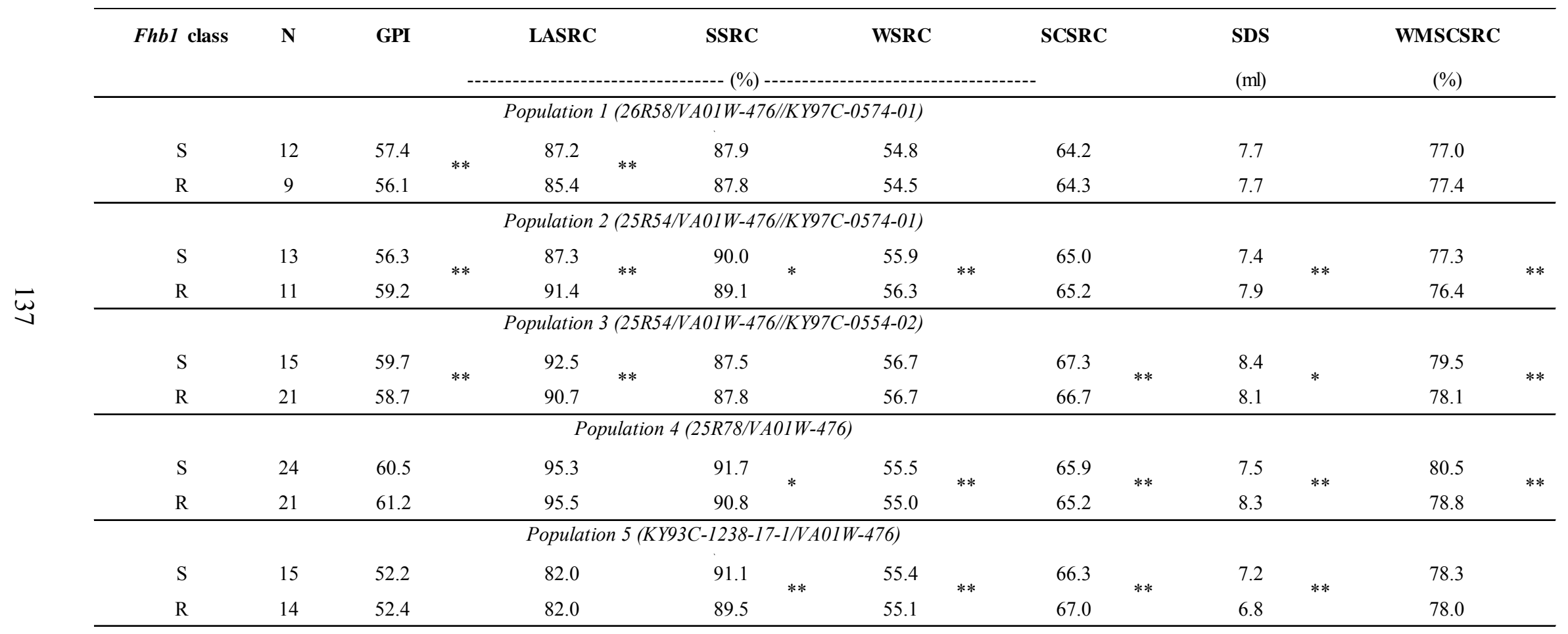

*, ** (Differences between $\mathrm{S}$ and $\mathrm{R}$ classes significant at $\mathrm{P}<0.05,0.01$ ) 
Table A.3.39: Mean whole grain hardness (WGH), whole grain protein (WGP), flour yield (FY), softness equivalent (SEQ) and flour protein (FP) for homozygous susceptible (S), resistant (R) and heterozygous (H) wheat lines at $Q F h s . n a u-2 D L$, Lexington 2010 and Princeton 2011.

\begin{tabular}{|c|c|c|c|c|c|c|c|c|c|c|c|}
\hline \multirow{2}{*}{$2 D L$ class } & $\mathbf{N}$ & \multicolumn{2}{|l|}{$\begin{array}{c}\text { WGH } \\
(0-100)\end{array}$} & WGP & & \multicolumn{2}{|l|}{ FY } & \multicolumn{2}{|l|}{ SEQ } & \multicolumn{2}{|l|}{ FP } \\
\hline & \multicolumn{11}{|c|}{ Population 1 (26R58/VA01W-476//KY97C-0574-01) } \\
\hline $\mathrm{S}$ & 9 & 24.6 & $\mathrm{~b}$ & 11.2 & $\mathrm{~b}$ & 68.5 & $\mathrm{a}$ & 56.6 & $\mathrm{~b}$ & 9.1 & $\mathrm{~b}$ \\
\hline $\mathrm{H}$ & 8 & 22.8 & a & 11.0 & a & 69.0 & $\mathrm{~b}$ & 56.1 & a & 9.0 & $\mathrm{a}$ \\
\hline \multirow[t]{2}{*}{$\mathrm{R}$} & 2 & 24.0 & $\mathrm{~b}$ & 11.3 & $\mathrm{~b}$ & 68.3 & $\mathrm{a}$ & 55.7 & $\mathrm{a}$ & 9.2 & $\mathrm{~b}$ \\
\hline & \multicolumn{11}{|c|}{ Population $2(25 R 54 / V A 01 W-476 / / K Y 97 C-0574-01)$} \\
\hline $\mathrm{S}$ & 4 & 21.2 & a & 10.3 & $\mathrm{a}$ & 67.3 & $\mathrm{c}$ & 56.5 & c & 8.3 & $\mathrm{a}$ \\
\hline $\mathrm{H}$ & 15 & 23.8 & $\mathrm{~b}$ & 10.9 & $\mathrm{~b}$ & 66.9 & $\mathrm{~b}$ & 53.9 & $\mathrm{~b}$ & 8.7 & $\mathrm{~b}$ \\
\hline \multirow[t]{2}{*}{$\mathrm{R}$} & 4 & 23.6 & $\mathrm{~b}$ & 11.1 & c & 66.5 & a & 50.4 & $\mathrm{a}$ & 8.9 & $\mathrm{c}$ \\
\hline & \multicolumn{11}{|c|}{ Population 3 (25R54/VA01W-476//KY97C-0554-02) } \\
\hline $\mathrm{S}$ & 17 & 25.1 & a & 11.1 & $\mathrm{c}$ & 69.7 & $\mathrm{a}$ & 51.8 & a & 9.5 & $\mathrm{c}$ \\
\hline $\mathrm{H}$ & 9 & 26.2 & b & 10.7 & b & 70.2 & $\mathrm{c}$ & 52.2 & b & 9.4 & $\mathrm{~b}$ \\
\hline $\mathrm{R}$ & 7 & 25.9 & $\mathrm{~b}$ & 10.5 & $\mathrm{a}$ & 70.0 & b & 53.7 & $\mathrm{c}$ & 9.0 & $\mathrm{a}$ \\
\hline
\end{tabular}

Means (by population) within the same column followed by different letters are significantly different at $\mathrm{P}<0.05$ 
Table A.3.39 (continued): Mean whole grain hardness (WGH), whole grain protein (WGP), flour yield (FY), softness equivalent (SEQ) and flour protein (FP) for homozygous susceptible (S), resistant (R) and heterozygous (H) wheat lines at QFhs.nau-2DL, Lexington 2010 and Princeton 2011.

\begin{tabular}{|c|c|c|c|c|c|c|c|c|c|c|c|}
\hline \multirow[t]{3}{*}{$2 D L$ class } & $\mathbf{N}$ & \multirow{2}{*}{\multicolumn{2}{|c|}{$\begin{array}{c}\text { WGH } \\
(0-100) \\
\end{array}$}} & WGP & & \multicolumn{2}{|l|}{$\mathbf{F Y}$} & \multicolumn{2}{|l|}{ SEQ } & \multicolumn{2}{|l|}{$\mathbf{F P}$} \\
\hline & & & & ------ & $-\cdots$ & 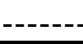 & 5 & ------- & $----\cdot$ & ---- & \\
\hline & \multicolumn{11}{|c|}{ Population $4(25 R 78 / V A 01 W-476)$} \\
\hline $\mathrm{S}$ & 12 & 26.6 & $\mathrm{ab}$ & 10.9 & a & 66.9 & $\mathrm{~b}$ & 58.1 & $\mathrm{~b}$ & 9.0 & a \\
\hline $\mathrm{H}$ & 20 & 26.2 & a & 11.0 & $\mathrm{~b}$ & 66.8 & $\mathrm{~b}$ & 57.7 & a & 9.0 & a \\
\hline \multirow[t]{2}{*}{$\mathrm{R}$} & 7 & 27.2 & $\mathrm{~b}$ & 10.8 & a & 66.6 & $\mathrm{a}$ & 57.7 & $a b$ & 8.9 & a \\
\hline & \multicolumn{11}{|c|}{ Population 5 (KY93C-1238-17-1/VA01W-476) } \\
\hline $\mathrm{S}$ & 6 & 21.8 & $\mathrm{ab}$ & 11.1 & $\mathrm{c}$ & 67.8 & $\mathrm{~b}$ & 57.3 & $\mathrm{a}$ & 8.7 & $\mathrm{~b}$ \\
\hline $\mathrm{H}$ & 15 & 21.3 & a & 10.8 & $\mathrm{~b}$ & 68.3 & $\mathrm{c}$ & 57.6 & a & 8.6 & b \\
\hline $\mathrm{R}$ & 8 & 22.3 & $\mathrm{~b}$ & 10.6 & $\mathrm{a}$ & 67.4 & $\mathrm{a}$ & 58.1 & $\mathrm{~b}$ & 8.4 & a \\
\hline
\end{tabular}

Means (by population) within the same column followed by different letters are significantly different at $\mathrm{P}<0.05$ 
Table A.3.40: Mean gluten performance index (GPI), flour lactic acid solvent retention capacity (LASRC), flour sucrose solvent retention capacity (SSRC), flour water solvent retention capacity (WSRC), sodium carbonate solvent retention capacity (SCSRC), wheat meal sedimentation test (SDS) and wheat meal SCSRC (WMSCSRC) for homozygous susceptible (S), resistant (R) and heterozygous (H) wheat lines at QFhs.nau-2DL, Lexington 2010 and Princeton 2011.

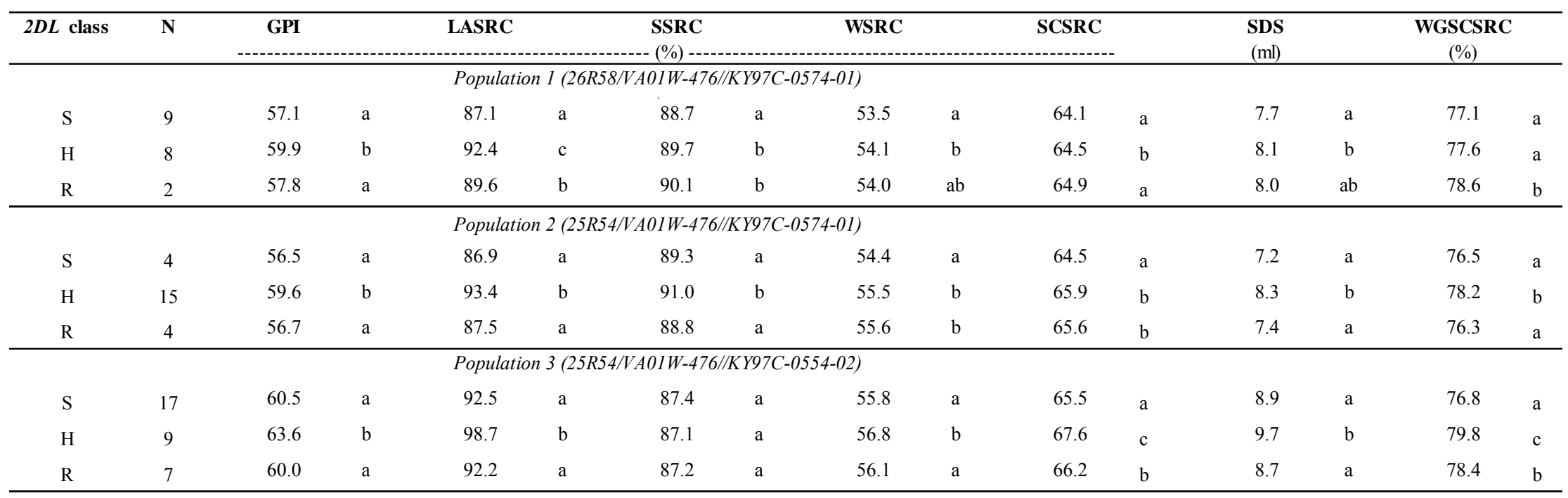

Means (by population) within the same column followed by different letters are significantly different at $\mathrm{P}<0.05$ 
Table A.3.40 (continued): Mean gluten performance index (GPI), flour lactic acid solvent retention capacity (LASRC),flour sucrose solvent retention capacity (SSRC), flour water solvent retention capacity (WSRC), sodium carbonate solvent retention capacity (SCSRC), wheat meal sedimentation test (SDS) and wheat meal SCSRC (WMSCSRC) for homozygous susceptible (S), resistant (R) and heterozygous (H) wheat lines at QFhs.nau-2DL, Lexington 2010 and Princeton 2011.

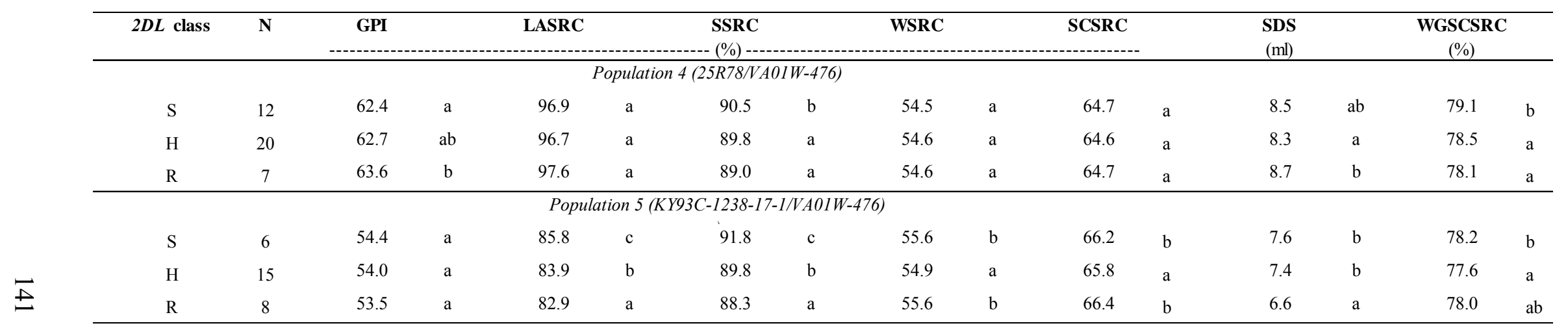

Means (by population) within the same column followed by different letters are significantly different at $\mathrm{P}<0.05$ 
Table A.3.41: Mean whole grain hardness (WGH), whole grain protein (WGP), flour yield (FY), softness equivalent (SEQ) and flour protein (FP) for homozygous susceptible (S), resistant (R) and heterozygous (H) wheat lines at QFhs.nau-2DL, Lexington 2010.

\begin{tabular}{|c|c|c|c|c|c|c|c|c|c|c|c|}
\hline $2 D L$ class & $\mathbf{N}$ & WGH & & WGP & & FY & & SEQ & & \multicolumn{2}{|l|}{$\mathbf{F P}$} \\
\hline & \multicolumn{11}{|c|}{ Population 1 (26R58/VA01W-476//KY97C-0574-01) } \\
\hline $\mathrm{S}$ & 9 & 20.4 & $\mathrm{~b}$ & 11.2 & a & 68.2 & $\mathrm{~b}$ & 58.3 & $\mathrm{c}$ & 8.9 & a \\
\hline $\mathrm{H}$ & 8 & 19.4 & a & 11.2 & $\mathrm{a}$ & 68.4 & $\mathrm{c}$ & 57.2 & $\mathrm{~b}$ & 8.9 & a \\
\hline $\mathrm{R}$ & 2 & 20.4 & $a b$ & 11.5 & $\mathrm{~b}$ & 67.6 & $\mathrm{a}$ & 56.2 & $\mathrm{a}$ & 9.3 & $\mathrm{~b}$ \\
\hline & \multicolumn{11}{|c|}{ Population $2(25 R 54 / V A 01 W-476 / / K Y 97 C-0574-01)$} \\
\hline $\mathrm{S}$ & 4 & 16.3 & $\mathrm{a}$ & 10.4 & $\mathrm{a}$ & 66.8 & $\mathrm{c}$ & 57.7 & c & 8.3 & a \\
\hline $\mathrm{H}$ & 15 & 18.9 & $\mathrm{~b}$ & 11.0 & $\mathrm{~b}$ & 66.3 & $\mathrm{~b}$ & 54.7 & $\mathrm{~b}$ & 8.7 & $\mathrm{~b}$ \\
\hline $\mathrm{R}$ & 4 & 18.9 & $\mathrm{~b}$ & 11.2 & $\mathrm{c}$ & 66.0 & $\mathrm{a}$ & 51.3 & $\mathrm{a}$ & 8.8 & $\mathrm{c}$ \\
\hline & \multicolumn{11}{|c|}{ Population 3 (25R54/VA01 W-476//KY97C-0554-02) } \\
\hline $\mathrm{S}$ & 17 & 21.4 & $\mathrm{a}$ & 11.4 & $\mathrm{c}$ & 69.3 & $\mathrm{a}$ & 51.9 & a & 9.6 & $\mathrm{~b}$ \\
\hline $\mathrm{H}$ & 9 & 22.5 & $\mathrm{~b}$ & 11.0 & $\mathrm{~b}$ & 69.8 & $\mathrm{c}$ & 52.5 & $\mathrm{~b}$ & 9.5 & $\mathrm{c}$ \\
\hline $\mathrm{R}$ & 7 & 22.4 & $\mathrm{~b}$ & 10.7 & $\mathrm{a}$ & 69.6 & $\mathrm{~b}$ & 54.2 & c & 9.0 & $\mathrm{a}$ \\
\hline
\end{tabular}

Means (by population) within the same column followed by different letters are significantly different at $\mathrm{P}<0.05$ 
Table A.3.41 (continued): Mean whole grain hardness (WGH), whole grain protein (WGP), flour yield (FY), softness equivalent (SEQ) and flour protein (FP) for homozygous susceptible (S), resistant (R) and heterozygous (H) wheat lines at QFhs.nau-2DL, Lexington 2010.

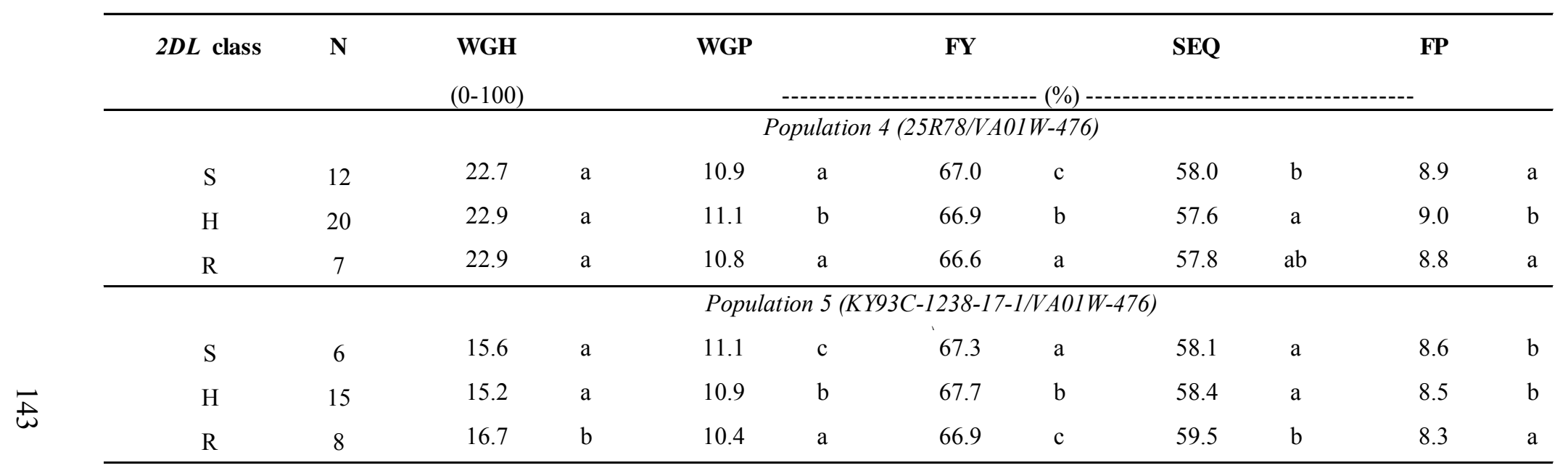

Means (by population) within the same column followed by different letters are significantly different at $\mathrm{P}<0.05$ 
Table A.3.42: Mean gluten performance index (GPI), flour lactic acid solvent retention capacity (LASRC),flour sucrose solvent retention capacity (SSRC), flour water solvent retention capacity (WSRC), sodium carbonate solvent retention capacity (SCSRC), estimated cookie diameter, wheat meal sedimentation test (SDS) and wheat meal SCSRC (WMSCSRC) for homozygous susceptible (S), resistant (R) and heterozygous (H) wheat lines at QFhs.nau-2DL, Lexington 2010.

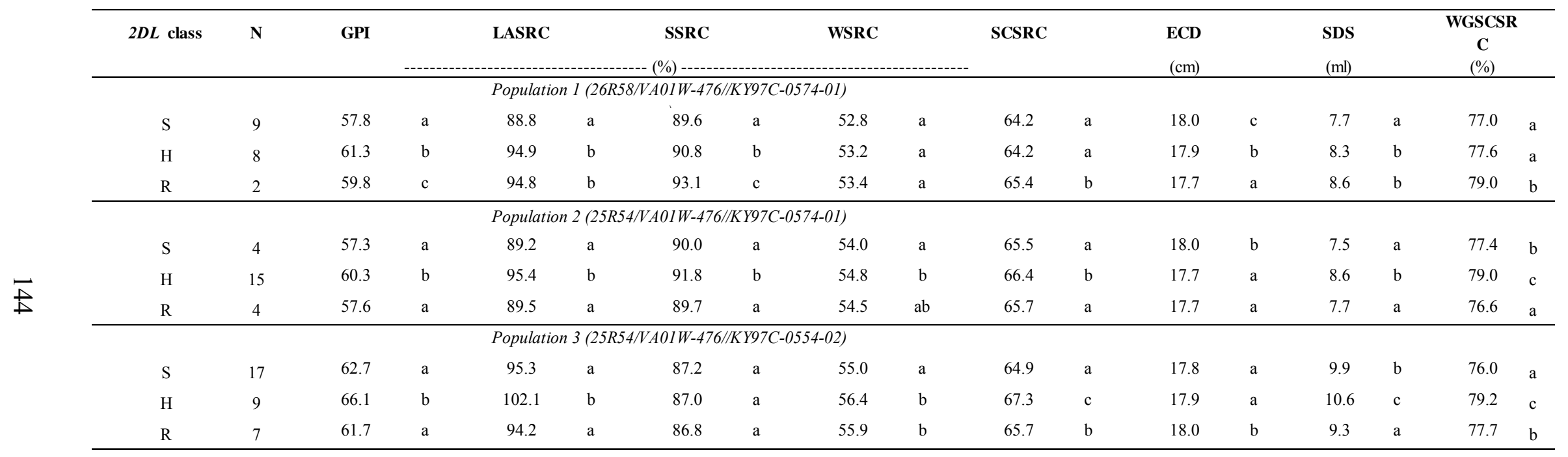

Means (by population) within the same column followed by different letters are significantly different at $\mathrm{P}<0.05$ 
Table A.3.42 (continued): Mean gluten performance index (GPI), flour lactic acid solvent retention capacity (LASRC), flour sucrose solvent retention capacity (SSRC), flour water solvent retention capacity (WSRC), sodium carbonate solvent retention capacity (SCSRC), estimated cookie diameter, wheat meal sedimentation test (SDS) and wheat meal SCSRC (WMSCSRC) for homozygous susceptible (S), resistant (R) and heterozygous (H) wheat lines at QFhs.nau-2DL, Lexington 2010.

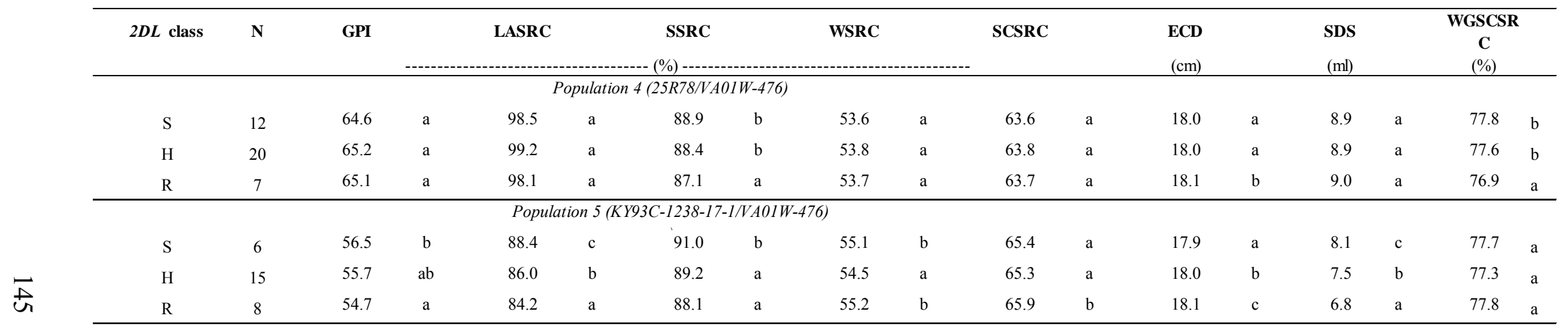

Means (by population) within the same column followed by different letters are significantly different at $\mathrm{P}<0.05$ 
Table A.3.43: Mean whole grain hardness (WGH), whole grain protein (WGP), flour yield (FY), softness equivalent (SEQ) and flour protein (FP) for homozygous susceptible (S), resistant (R) and heterozygous (H) wheat lines at QFhs.nau-2DL, Princeton 2011.

\begin{tabular}{|c|c|c|c|c|c|c|c|c|c|c|c|}
\hline $2 D L$ class & $\mathbf{N}$ & WGH & & WGP & & FY & & SEQ & & \multicolumn{2}{|l|}{ FP } \\
\hline & \multicolumn{11}{|c|}{ Population 1 (26R58/VA01W-476//KY97C-0574-01) } \\
\hline S & 9 & 28.8 & $\mathrm{c}$ & 11.2 & $\mathrm{~b}$ & 68.8 & a & 54.8 & $\mathrm{a}$ & 9.3 & $\mathrm{~b}$ \\
\hline $\mathrm{H}$ & 8 & 26.2 & a & 10.9 & a & 69.5 & $\mathrm{~b}$ & 55.0 & a & 9.1 & $\mathrm{a}$ \\
\hline $\mathrm{R}$ & 2 & 27.6 & $\mathrm{~b}$ & 11.1 & $\mathrm{ab}$ & 69.0 & a & 55.2 & a & 9.1 & a \\
\hline & \multicolumn{11}{|c|}{ Population $2(25 R 54 / V A 01 W-476 / / K Y 97 C-0574-01)$} \\
\hline $\mathrm{S}$ & 4 & 26.1 & a & 10.1 & $\mathrm{a}$ & 67.8 & $\mathrm{~b}$ & 55.5 & $\mathrm{c}$ & 8.4 & $\mathrm{a}$ \\
\hline $\mathrm{H}$ & 15 & 28.7 & $\mathrm{~b}$ & 10.8 & $\mathrm{~b}$ & 67.4 & $\mathrm{~b}$ & 53.1 & $\mathrm{~b}$ & 8.7 & $\mathrm{~b}$ \\
\hline $\mathrm{R}$ & 4 & 28.2 & $\mathrm{~b}$ & 11.1 & $\mathrm{c}$ & 66.9 & $\mathrm{a}$ & 49.5 & a & 9.0 & $\mathrm{c}$ \\
\hline & \multicolumn{11}{|c|}{ Population 3 (25R54/VA01W-476//KY97C-0554-02) } \\
\hline $\mathrm{S}$ & 17 & 28.9 & a & 10.8 & $\mathrm{~b}$ & 70.0 & a & 51.6 & a & 9.4 & $\mathrm{~b}$ \\
\hline $\mathrm{H}$ & 9 & 29.8 & $\mathrm{~b}$ & 10.4 & $\mathrm{a}$ & 70.7 & $\mathrm{c}$ & 51.9 & $\mathrm{a}$ & 9.3 & $\mathrm{~b}$ \\
\hline $\mathrm{R}$ & 7 & 29.5 & $\mathrm{ab}$ & 10.3 & $\mathrm{a}$ & 70.4 & $\mathrm{~b}$ & 53.3 & $\mathrm{~b}$ & 9.0 & a \\
\hline
\end{tabular}

Means (by population) within the same column followed by different letters are significantly different at $\mathrm{P}<0.05$ 
Table A.3.43 (continued): Mean whole grain hardness (WGH), whole grain protein (WGP), flour yield (FY), softness equivalent (SEQ) and flour protein (FP) for homozygous susceptible (S), resistant (R) and heterozygous (H) wheat lines at QFhs.nau-2DL, Princeton 2011.

\begin{tabular}{|c|c|c|c|c|c|c|c|c|c|c|c|}
\hline \multirow[t]{2}{*}{$2 D L$ class } & $\mathbf{N}$ & WGH & & WGP & & $\mathbf{F Y}$ & & SEQ & & FP & \\
\hline & \multicolumn{11}{|c|}{ Population 4 (25R78/VA01W-476) } \\
\hline $\mathrm{S}$ & 12 & 30.5 & $\mathrm{~b}$ & 10.8 & a & 66.8 & a & 58.3 & $\mathrm{~b}$ & 9.0 & $\mathrm{a}$ \\
\hline $\mathrm{H}$ & 20 & 29.4 & $\mathrm{a}$ & 10.8 & $\mathrm{a}$ & 66.7 & a & 57.7 & a & 8.9 & $\mathrm{a}$ \\
\hline \multirow[t]{2}{*}{$\mathrm{R}$} & 7 & 31.5 & $\mathrm{~b}$ & 10.8 & $\mathrm{a}$ & 66.6 & a & 57.7 & $\mathrm{ab}$ & 9.0 & $\mathrm{a}$ \\
\hline & \multicolumn{11}{|c|}{ Population 5 (KY93C-1238-17-1/VA01W-476) } \\
\hline $\mathrm{S}$ & 6 & 27.9 & a & 11.1 & $\mathrm{~b}$ & 68.2 & $\mathrm{a}$ & 56.5 & a & 8.8 & b \\
\hline $\mathrm{H}$ & 15 & 27.4 & $\mathrm{a}$ & 10.8 & $\mathrm{a}$ & 68.9 & $\mathrm{~b}$ & 56.8 & $\mathrm{a}$ & 8.6 & $\mathrm{~b}$ \\
\hline $\mathrm{R}$ & 8 & 27.9 & $\mathrm{a}$ & 10.7 & $\mathrm{a}$ & 67.9 & $\mathrm{a}$ & 56.7 & $\mathrm{a}$ & 8.5 & $\mathrm{a}$ \\
\hline
\end{tabular}

Means (by population) within the same column followed by different letters are significantly different at $\mathrm{P}<0.05$ 
Table A.3.44: Mean gluten performance index (GPI), flour lactic acid solvent retention capacity (LASRC),flour sucrose solvent retention capacity (SSRC), flour water solvent retention capacity (WSRC), sodium carbonate solvent retention capacity (SCSRC), wheat meal sedimentation test (SDS) and wheat meal SCSRC (WMSCSRC) for homozygous susceptible (S), resistant (R) and heterozygous (H) wheat lines at QFhs.nau-2DL, Princeton 2011.

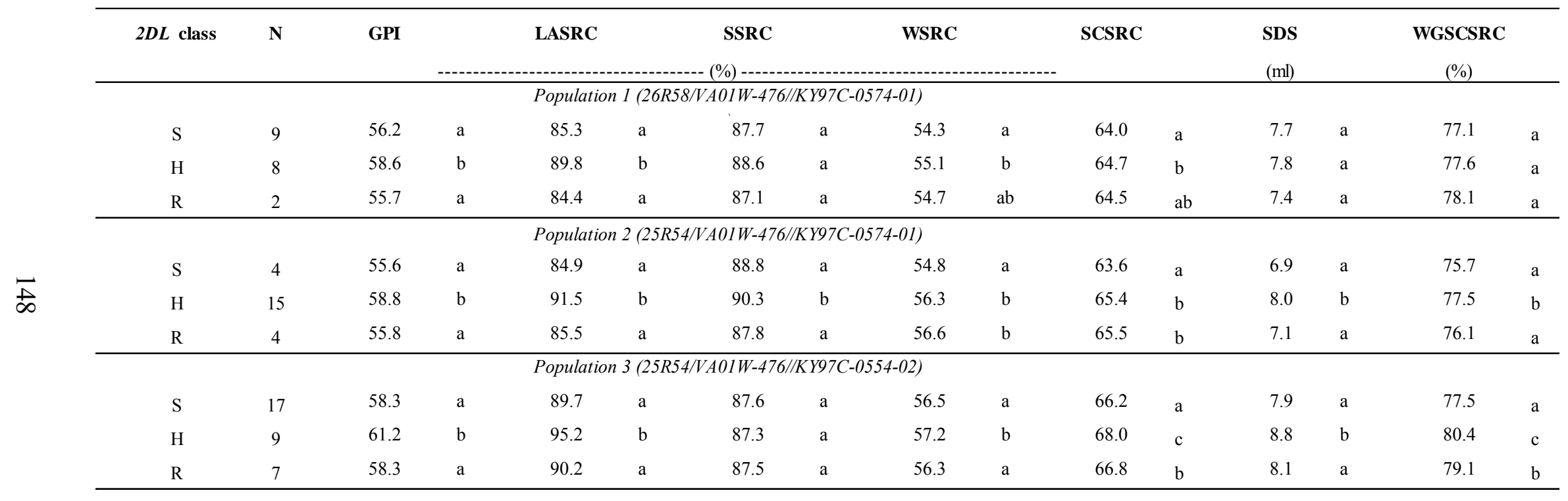

Means (by population) within the same column followed by different letters are significantly different at $\mathrm{P}<0.05$ 
Table A.3.44 (continued): Mean gluten performance index (GPI), flour lactic acid solvent retention capacity (LASRC),flour sucrose solvent retention capacity (SSRC), flour water solvent retention capacity (WSRC), sodium carbonate solvent retention capacity (SCSRC), wheat meal sedimentation test (SDS) and wheat meal SCSRC (WMSCSRC) for homozygous susceptible (S), resistant (R) and heterozygous (H) wheat lines at QFhs.nau-2DL, Princeton 2011.

\begin{tabular}{|c|c|c|c|c|c|c|c|c|c|c|c|c|c|c|c|}
\hline $2 D L$ class & $\mathbf{N}$ & GPI & & LASRC & ---- & $\begin{array}{l}\text { SSRC } \\
\%)\end{array}$ & & WSRC & & SCSRC & & \multirow[t]{2}{*}{$\begin{array}{l}\text { SDS } \\
(\mathrm{ml}) \\
\end{array}$} & & \multicolumn{2}{|c|}{$\begin{array}{c}\text { WGSCSRC } \\
(\%) \\
\end{array}$} \\
\hline \multicolumn{12}{|c|}{ Population $4(25 R 78 / V A 01 W-476)$} & & & & \\
\hline $\mathrm{S}$ & 12 & 60.3 & a & 95.3 & $\mathrm{ab}$ & 92.2 & $\mathrm{~b}$ & 55.4 & a & 65.9 & $\mathrm{~b}$ & 8.1 & $\mathrm{~b}$ & 80.4 & $\mathrm{~b}$ \\
\hline $\mathrm{H}$ & 20 & 60.2 & a & 94.2 & a & 91.1 & a & 55.3 & a & 65.4 & a & 7.7 & $\mathrm{a}$ & 79.4 & a \\
\hline $\mathrm{R}$ & 7 & 62.0 & $\mathrm{~b}$ & 97.0 & $\mathrm{~b}$ & 90.8 & a & 55.4 & a & 65.6 & $\mathrm{ab}$ & 8.3 & $\mathrm{~b}$ & 79.4 & a \\
\hline \multicolumn{16}{|c|}{ Population 5 (KY93C-1238-17-1/VA01W-476) } \\
\hline $\mathrm{S}$ & 6 & 52.2 & a & 83.2 & $\mathrm{~b}$ & 92.5 & $\mathrm{c}$ & 56.1 & $\mathrm{~b}$ & 67.0 & $\mathrm{~b}$ & 7.1 & $\mathrm{~b}$ & 78.7 & $\mathrm{~b}$ \\
\hline $\mathrm{H}$ & 15 & 52.2 & a & 81.8 & a & 90.3 & $\mathrm{~b}$ & 55.4 & a & 66.3 & a & 7.3 & $\mathrm{~b}$ & 77.9 & a \\
\hline $\mathrm{R}$ & 8 & 52.4 & a & 81.5 & a & 88.6 & a & 56.0 & $\mathrm{~b}$ & 67.0 & $\mathrm{~b}$ & 6.5 & a & 78.1 & $\mathrm{ab}$ \\
\hline
\end{tabular}

Means (by population) within the same column followed by different letters are significantly different at $\mathrm{P}<0.05$ 
Table A.3.45: Mean whole grain hardness (WGH), whole grain protein (WGP), flour yield (FY), softness equivalent (SEQ) and flour protein (FP) for wheat lines in each QTL combination class when all populations were combined, Lexington 2010 and Princeton 2011.

\begin{tabular}{|c|c|c|c|c|c|c|c|c|c|c|c|}
\hline \multirow{3}{*}{$\begin{array}{c}\begin{array}{c}F h b 1 \text { and } 2 D L \\
\text { combination class }\end{array} \\
R H\end{array}$} & \multirow{3}{*}{$\begin{array}{l}\mathbf{N} \\
29\end{array}$} & \multirow{2}{*}{\multicolumn{2}{|c|}{$\begin{array}{c}\text { WGH } \\
(0-100)\end{array}$}} & \multirow{2}{*}{\multicolumn{2}{|c|}{ WGP }} & \multicolumn{2}{|l|}{ FY } & \multicolumn{2}{|l|}{ SEQ } & \multicolumn{2}{|l|}{ FP } \\
\hline & & & & & & ------ & & ------ & -- & - & --- \\
\hline & & 23.6 & $\mathrm{a}$ & 10.9 & $\mathrm{bc}$ & 67.7 & $\mathrm{~b}$ & 55.8 & $\mathrm{c}$ & 8.8 & $\mathrm{a}$ \\
\hline $\mathrm{RR}$ & 15 & 25.4 & d & 10.7 & $\mathrm{a}$ & 67.2 & $\mathrm{a}$ & 55.9 & $\mathrm{c}$ & 8.8 & $\mathrm{a}$ \\
\hline $\mathrm{RS}$ & 29 & 24.6 & bc & 11.0 & $\mathrm{~d}$ & 68.6 & $d$ & 54.5 & $\mathrm{a}$ & 9.2 & $\mathrm{c}$ \\
\hline SH & 38 & 24.6 & $\mathrm{c}$ & 10.9 & $\mathrm{c}$ & 68.0 & $\mathrm{c}$ & 55.9 & $\mathrm{c}$ & 8.9 & $\mathrm{~b}$ \\
\hline SR & 13 & 24.0 & $a b$ & 10.8 & $a b$ & 68.5 & $d$ & 55.4 & $\mathrm{~b}$ & 8.9 & $a b$ \\
\hline SS & 19 & 24.6 & $\mathrm{bc}$ & 10.9 & $\mathrm{~cd}$ & 68.0 & $\mathrm{c}$ & 56.5 & $\mathrm{~d}$ & 9.0 & $\mathrm{~b}$ \\
\hline
\end{tabular}

Means within the same column followed by different letters are significantly different at $\mathrm{P}<0.05$ 
Table A.3.46: Mean gluten performance index (GPI), flour lactic acid solvent retention capacity (LASRC), flour sucrose solvent retention capacity (SSRC), flour water solvent retention capacity (WSRC), flour sodium carbonate solvent retention capacity (SCSRC), wheat meal sedimentation test (SDS) and wheat meal SCSRC (WMSCSRC) for wheat lines in each QTL combination class when all populations were combined, Lexington 2010 and Princeton 2011.

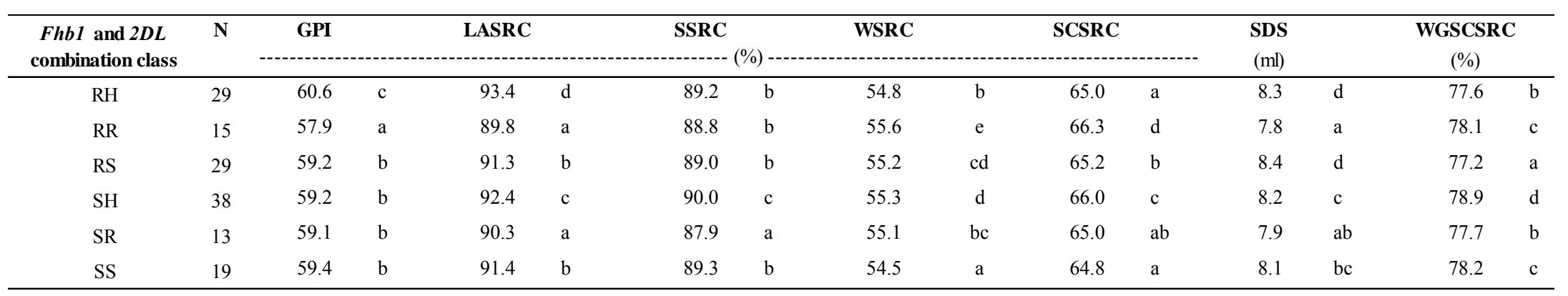

Means within the same column followed by different letters are significantly different at $\mathrm{P}<0.05$ 
Table A.3.47: Heritabilities and their $90 \%$ confidence intervals in parentheses based on 3 year ANOVA of five wheat populations. Traits evaluated were: yield, test weight (TWT), heading date (HD) and plant height, Lexington 2010 and 2011, and Princeton 2011.

\begin{tabular}{cccccccccc}
\hline & \multicolumn{2}{c}{ Yield } & \multicolumn{2}{c}{ TWT } & \multicolumn{2}{c}{ HD } & \multicolumn{2}{c}{ Height } \\
\hline Overall & 0.67 & $(0.72-0.74)$ & 0.85 & $(0.88-0.82)$ & 0.78 & $(0.81-0.73)$ & 0.91 & $(0.93-0.90)$ \\
Population 1 & 0.79 & $(0.87-0.63)$ & 0.90 & $(0.94-0.83)$ & 0.83 & $(0.90-0.71)$ & 0.90 & $(0.94-0.83)$ \\
Population 2 & 0.84 & $(0.91-0.74)$ & 0.91 & $(0.94-0.85)$ & 0.87 & $(0.92-0.79)$ & 0.92 & $(0.95-0.87)$ \\
Population 3 & 0.83 & $(0.88-0.74)$ & 0.84 & $(0.89-0.76)$ & 0.58 & $(0.71-0.38)$ & 0.91 & $(0.94-0.87)$ \\
Population 4 & 0.46 & $(0.61-0.23)$ & 0.89 & $(0.92-0.84)$ & 0.53 & $(0.66-0.33)$ & 0.92 & $(0.94-0.89)$ \\
Population 5 & 0.41 & $(0.61-0.08)$ & 0.78 & $(0.85-0.66)$ & 0.88 & $(0.92-0.81)$ & 0.94 & $(0.96-0.90)$ \\
\hline
\end{tabular}


Table A.3.48: Heritabilities and their 90\% confidence intervals in parentheses based on 2 year ANOVA of five wheat populations. Traits evaluated were: whole grain hardness (WGH), whole grain protein (WGP), flour yield (FY), softness equivalent (SEQ) and flour protein (FP), Lexington 2010 and Princeton 2011.

\begin{tabular}{cccccccccccc}
\hline & \multicolumn{2}{c}{ WGH } & \multicolumn{2}{c}{ WGP } & \multicolumn{2}{c}{ FY } & \multicolumn{2}{c}{ SEQ } & \multicolumn{3}{c}{ FP } \\
\hline Overall & 0.81 & $(0.84-0.76)$ & 0.81 & $(0.85-0.77)$ & 0.94 & $(0.95-0.92)$ & 0.95 & $(0.96-0.94)$ & 0.91 & $(0.92-0.88)$ \\
Population 1 & 0.73 & $(0.85-0.52)$ & 0.72 & $(0.85-0.50)$ & 0.85 & $(0.92-0.74)$ & 0.93 & $(0.96-0.88)$ & 0.85 & $(0.92-0.73)$ \\
Population 2 & 0.94 & $(0.97-0.90)$ & 0.95 & $(0.97-0.91)$ & 0.90 & $(0.94-0.84)$ & 0.98 & $(0.99-0.96)$ & 0.96 & $(0.98-0.93)$ \\
Population 3 & 0.80 & $(0.87-0.69)$ & 0.92 & $(0.95-0.88)$ & 0.95 & $(0.97-0.93)$ & 0.98 & $(0.99-0.96)$ & 0.95 & $(0.97-0.92)$ \\
Population 4 & 0.80 & $(0.86-0.70)$ & 0.80 & $(0.86-0.70)$ & 0.88 & $(0.92-0.82)$ & 0.91 & $0.94-0.86)$ & 0.79 & $(0.86-0.69)$ \\
Population 5 & 0.89 & $(0.93-0.82)$ & 0.64 & $(0.78-0.41)$ & 0.93 & $(0.96-0.88)$ & 0.89 & $(0.93-0.82)$ & 0.78 & $(0.86-0.63)$ \\
\hline
\end{tabular}


Table A.3.49: Heritabilities and their 90\% confidence intervals in parentheses based on 2 year ANOVA of five wheat populations. Traits evaluated were: gluten performance index (GPI), flour lactic acid solvent retention capacity (LASRC), flour sucrose solvent retention capacity (SSRC), flour water solvent retention capacity (WSRC) and flour sodium carbonate solvent retention capacity (SCSRC), Lexington 2010 and Princeton 2011.

\begin{tabular}{cccccccccccc}
\hline & \multicolumn{2}{c}{ GPI } & \multicolumn{2}{c}{ LASRC } & \multicolumn{2}{c}{ SSRC } & \multicolumn{3}{c}{ WSRC } & \multicolumn{2}{c}{ SCSRC } \\
\hline Overall & 0.93 & $(0.94-0.91)$ & 0.95 & $(0.96-0.93)$ & 0.68 & $(0.74-0.61)$ & 0.89 & $(0.91-0.87)$ & 0.84 & $(0.87-0.80)$ \\
Population 1 & 0.95 & $(0.97-0.91)$ & 0.93 & $(0.96-0.87)$ & 0.50 & $(0.72-0.10)$ & 0.83 & $(0.90-0.69)$ & 0.84 & $(0.91-0.71)$ \\
Population 2 & 0.95 & $(0.97-0.92)$ & 0.95 & $(0.97-0.92)$ & 0.54 & $(0.73-0.21)$ & 0.85 & $(0.91-0.73)$ & 0.75 & $(0.85-0.56)$ \\
Population 3 & 0.93 & $(0.95-0.89)$ & 0.96 & $(0.97-0.94)$ & 0.86 & $(0.92-0.79)$ & 0.92 & $(0.95-0.87)$ & 0.93 & $(0.96-0.90)$ \\
Population 4 & 0.79 & $(0.86-0.69)$ & 0.82 & $(0.88-0.74)$ & 0.76 & $(0.84-0.74)$ & 0.80 & $(0.87-0.71)$ & 0.89 & $(0.93-0.84)$ \\
Population 5 & 0.80 & $(0.88-0.67)$ & 0.89 & $(0.93-0.82)$ & 0.82 & $(0.89-0.70)$ & 0.84 & $(0.90-0.73)$ & 0.85 & $(0.91-0.75)$ \\
\hline
\end{tabular}


Table A.3.50: Genotype mean Pearson correlation coefficients between test weight (TWT) whole grain hardness (WGH), whole grain protein (WGP), flour yield (FY), softness equivalent (SEQ) and flour protein (FP), gluten performance index (GPI), flour lactic acid solvent retention capacity (LASRC), flour sucrose solvent retention capacity (SSRC), flour water solvent retention capacity (WSRC) and flour sodium carbonate solvent retention capacity (SCSRC), Lexington 2010 and Princeton 2011.

\begin{tabular}{|c|c|c|c|c|c|c|c|c|c|c|c|}
\hline & WGP & WGH & FY & SEQ & FP & LASRC & SSRC & ECD & WSRC & SCSRC & GPI \\
\hline \multirow{2}{*}{ TWT } & 0.37 & -0.12 & -0.04 & -0.30 & 0.12 & -0.22 & 0.06 & -0.33 & 0.09 & -0.02 & -0.24 \\
\hline & $<.0001$ & 0.1526 & 0.6544 & 0.0001 & 0.1371 & 0.005 & 0.4931 & $<.0001$ & 0.2742 & 0.7792 & 0.0029 \\
\hline \multirow{2}{*}{ WGP } & & 0.37 & -0.12 & -0.39 & 0.79 & 0.23 & 0.25 & -0.54 & 0.02 & -0.13 & 0.20 \\
\hline & & $<.0001$ & 0.1335 & $<.0001$ & $<.0001$ & 0.004 & 0.0018 & $<.0001$ & 0.8418 & 0.1168 & 0.011 \\
\hline \multirow{2}{*}{ WGH } & & & -0.21 & -0.27 & 0.60 & 0.55 & 0.06 & -0.24 & 0.27 & 0.19 & 0.53 \\
\hline & & & 0.009 & 0.0006 & $<.0001$ & $<.0001$ & 0.4354 & 0.0021 & 0.0008 & 0.0193 & $<.0001$ \\
\hline \multirow{2}{*}{ FY } & & & & -0.34 & 0.17 & -0.26 & -0.56 & 0.03 & 0.02 & -0.02 & -0.15 \\
\hline & & & & $<.0001$ & 0.0301 & 0.0013 & $<.0001$ & 0.7349 & 0.7664 & 0.8185 & 0.0671 \\
\hline \multirow{2}{*}{ SEQ } & & & & & -0.61 & -0.09 & 0.28 & 0.73 & -0.53 & -0.32 & -0.11 \\
\hline & & & & & $<.0001$ & 0.2428 & 0.0004 & $<.0001$ & $<.0001$ & $<.0001$ & 0.1839 \\
\hline \multirow{2}{*}{ FP } & & & & & & 0.44 & -0.03 & -0.53 & 0.23 & 0.06 & 0.45 \\
\hline & & & & & & $<.0001$ & 0.6778 & $<.0001$ & 0.0038 & 0.4383 & $<.0001$ \\
\hline \multirow{2}{*}{ LASRC } & & & & & & & 0.23 & -0.19 & 0.26 & 0.19 & 0.96 \\
\hline & & & & & & & 0.0035 & 0.0196 & 0.0008 & 0.0178 & $<.0001$ \\
\hline \multirow{2}{*}{ SSRC } & & & & & & & & -0.39 & 0.14 & 0.25 & -0.01 \\
\hline & & & & & & & & $<.0001$ & 0.0716 & 0.0015 & 0.9359 \\
\hline \multirow{2}{*}{ ECD } & & & & & & & & & -0.50 & -0.38 & -0.05 \\
\hline & & & & & & & & & $<.0001$ & $<.0001$ & 0.5261 \\
\hline \multirow{2}{*}{ WSRC } & & & & & & & & & & 0.90 & 0.11 \\
\hline & & & & & & & & & & $<.0001$ & 0.1688 \\
\hline \multirow{2}{*}{ SCSRC } & & & & & & & & & & & -0.01 \\
\hline & & & & & & & & & & & 0.9479 \\
\hline
\end{tabular}


Table A.3.51: Mean Fusarium damaged kernels (FDK), deoxynivalenol level (DON), FHB index, severity, incidence, FHB rating, heading date and plant height for wheat lines homozygous for resistant (R) and susceptible (S) alleles at Fhb1, Lexington 2011 (Increase lines included).

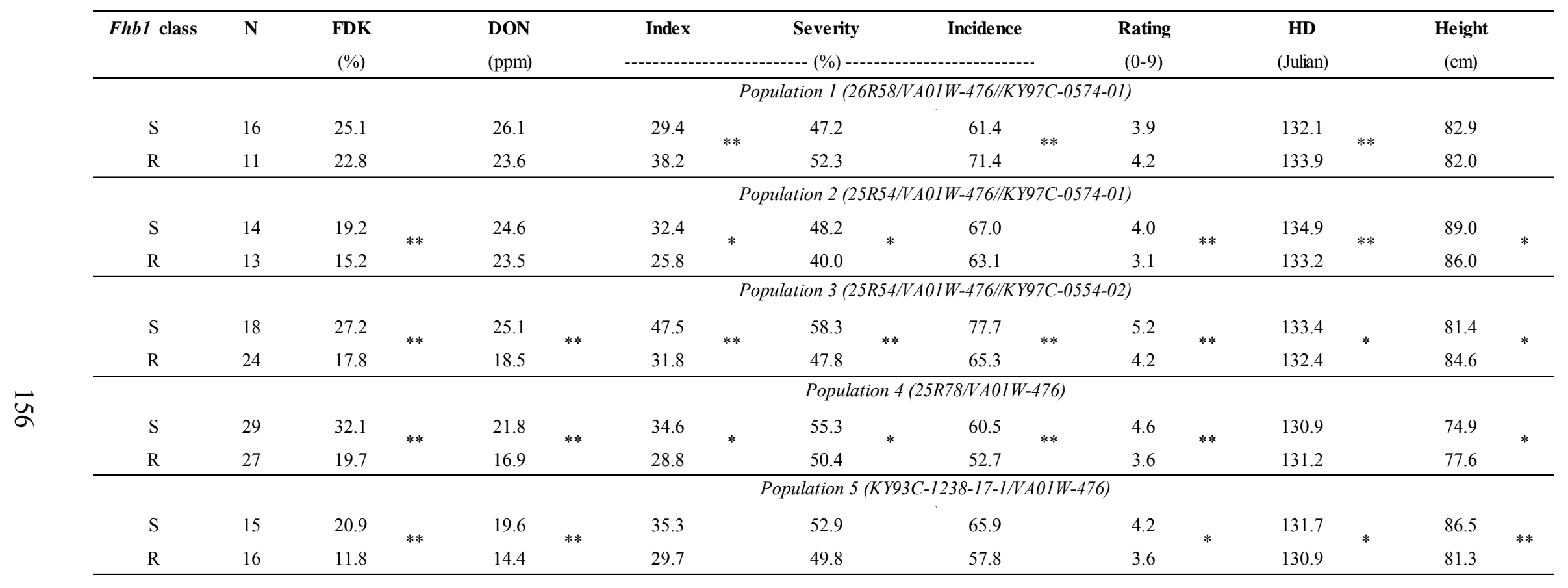

*, ** (Differences between $\mathrm{S}$ and $\mathrm{R}$ classes significant at $\mathrm{P}<0.05,0.01$ ) 
Table A.3.52: Mean yield, test weight (TWT), plant height, heading date (HD) and FHB rating measured in plots for wheat lines homozygous for resistant (R) and susceptible (S) alleles at Fhbl, Lexington 2011 (Increase lines included).

\begin{tabular}{|c|c|c|c|c|c|c|c|c|c|c|}
\hline Fhb1 class & $\mathbf{N}$ & $\begin{array}{c}\text { Yield } \\
\text { (kg/ha) }\end{array}$ & & $\begin{array}{l}\text { TWT } \\
(\mathrm{kg} / \mathrm{hl})\end{array}$ & & $\begin{array}{c}\text { Height } \\
\text { (cm) }\end{array}$ & $\begin{array}{c}\text { HD } \\
\text { (Julian) }\end{array}$ & & $\begin{array}{c}\text { Rating } \\
(0-9)\end{array}$ & \\
\hline \multirow[b]{3}{*}{$\mathrm{R}$} & \multicolumn{10}{|c|}{ Population 1 (26R58/VA01 W-476//KY97C-0574-01) } \\
\hline & \multirow{2}{*}{$\begin{array}{l}16 \\
11 \\
\end{array}$} & \multirow{2}{*}{\multicolumn{2}{|c|}{$\begin{array}{l}3844 \\
3760 \\
\end{array}$}} & \multirow{2}{*}{\multicolumn{2}{|c|}{$\begin{array}{l}68.6 \\
68.1\end{array}$}} & 86.1 & 130.2 & \multirow{2}{*}{$* *$} & 4.0 & \multirow{2}{*}{ ** } \\
\hline & & & & & & 81.5 & 131.5 & & 3.0 & \\
\hline & \multicolumn{10}{|c|}{ Population $2(25 R 54 / V A 01 W-476 / / K Y 97 C-0574-01)$} \\
\hline $\mathrm{S}$ & 14 & 3681 & \multirow{2}{*}{$* *$} & 71.3 & \multirow{2}{*}{$* *$} & 91.4 & 133.4 & \multirow{2}{*}{$* *$} & 2.6 & \multirow{2}{*}{ * } \\
\hline $\mathrm{R}$ & 13 & 4035 & & 71.9 & & 86.9 & 131.7 & & 2.3 & \\
\hline & \multicolumn{10}{|c|}{ Population $3(25 R 54 / V A 01 \mathrm{~W}-476 / / K Y 97 C-0554-02)$} \\
\hline $\mathrm{S}$ & 18 & 3676 & \multirow{2}{*}{$* *$} & 67.7 & \multirow{2}{*}{$* *$} & 80.0 & 131.4 & \multirow{2}{*}{$* *$} & 4.2 & \multirow{2}{*}{$* *$} \\
\hline $\mathrm{R}$ & 24 & 4068 & & 70.4 & & 81.9 & 131.9 & & 3.1 & \\
\hline & \multicolumn{10}{|c|}{ Population $4(25 R 78 / V A 01 W-476)$} \\
\hline $\mathrm{S}$ & 29 & 3305 & \multirow{2}{*}{$* *$} & 67.6 & \multirow{2}{*}{$* *$} & 76.0 & 129.8 & \multirow{2}{*}{$* *$} & 2.6 & \multirow{2}{*}{ ** } \\
\hline \multirow[t]{2}{*}{$\mathrm{R}$} & 27 & 3522 & & 68.8 & & 74.4 & 130.3 & & 1.6 & \\
\hline & \multicolumn{10}{|c|}{ Population 5 (KY93C-1238-17-1/VA01W-476) } \\
\hline $\mathrm{S}$ & 15 & 3970 & & 69.6 & \multirow{2}{*}{$* *$} & 82.2 & 130.5 & \multirow{2}{*}{ * } & 3.7 & \multirow{2}{*}{ ** } \\
\hline $\mathrm{R}$ & 16 & 4106 & & 70.9 & & 82.1 & 129.7 & & 2.6 & \\
\hline
\end{tabular}

*,** (Differences between $\mathrm{S}$ and $\mathrm{R}$ classes significant at $\mathrm{P}<0.05,0.01$ ) 
Table A.3.53: Mean yield, test weight (TWT), plant height, heading date (HD) and FHB rating measured in plots for wheat lines homozygous for resistant (R) and susceptible (S) alleles at Fhb1, Princeton 2011 (Increase lines included).

\begin{tabular}{|c|c|c|c|c|c|c|c|c|c|c|}
\hline Fhb1 class & $\mathbf{N}$ & $\begin{array}{c}\text { Yield } \\
(\mathrm{kg} / \mathrm{ha})\end{array}$ & & $\begin{array}{l}\text { TWT } \\
(\mathrm{kg} / \mathrm{hl})\end{array}$ & & $\begin{array}{l}\text { Height } \\
(\mathrm{cm})\end{array}$ & $\begin{array}{c}\text { HD } \\
\text { (Julian) }\end{array}$ & & $\begin{array}{c}\text { Rating } \\
(0-9)\end{array}$ & \\
\hline & \multicolumn{10}{|c|}{ Population 1 (26R58/VA01W-476//KY97C-0574-01) } \\
\hline $\mathrm{S}$ & 16 & 4447 & & 71.0 & & 90.4 & 121.1 & \multirow{2}{*}{$* *$} & 3.0 & \multirow{2}{*}{ ** } \\
\hline $\mathrm{R}$ & 11 & 4270 & & 70.4 & & 91.4 & 122.8 & & 2.1 & \\
\hline & \multicolumn{10}{|c|}{ Population $2(25 R 54 / V A 01$ W-476//KY97C-0574-01) } \\
\hline $\mathrm{S}$ & 14 & 4261 & \multirow{2}{*}{$* *$} & 72.0 & & 95.7 & 124.2 & \multirow{2}{*}{ ** } & \multirow{2}{*}{\multicolumn{2}{|c|}{$\begin{array}{l}2.3 \\
2.3 \\
\end{array}$}} \\
\hline $\mathrm{R}$ & 13 & 4453 & & 72.7 & & 94.0 & 122.1 & & & \\
\hline & \multicolumn{10}{|c|}{ Population 3 (25R54/VA01W-476//KY97C-0554-02) } \\
\hline $\mathrm{S}$ & 18 & 4182 & \multirow{2}{*}{ ** } & 70.4 & \multirow{2}{*}{ ** } & 85.2 & 122.9 & \multirow{2}{*}{ * } & 4.0 & \multirow{2}{*}{ ** } \\
\hline $\mathrm{R}$ & 24 & 4453 & & 72.2 & & 86.4 & 122.1 & & 3.1 & \\
\hline & \multicolumn{10}{|c|}{ Population $4(25 R 78 / V A 01 W-476)$} \\
\hline $\mathrm{S}$ & 29 & 3823 & & 69.9 & \multirow{2}{*}{ ** } & 81.0 & 121.4 & & 1.2 & \multirow{2}{*}{$* *$} \\
\hline $\mathrm{R}$ & 27 & 3842 & & 71.1 & & 81.6 & 121.1 & & 0.4 & \\
\hline & \multicolumn{10}{|c|}{ Population 5 (KY93C-1238-17-1/VA01W-476) } \\
\hline $\mathrm{S}$ & 15 & 4290 & \multirow{2}{*}{$* *$} & 72.5 & \multirow{2}{*}{ * } & 95.2 & 121.8 & & 1.6 & \multirow{2}{*}{ ** } \\
\hline $\mathrm{R}$ & 16 & 4464 & & 73.1 & & 94.0 & 121.2 & & 1.0 & \\
\hline
\end{tabular}

*, ** (Differences between $\mathrm{S}$ and $\mathrm{R}$ classes significant at $\mathrm{P}<0.05,0.01$ ) 
Table A.3.54: Mean whole grain hardness (WGH), whole grain protein (WGP), flour yield (FY), softness equivalent (SEQ) and flour protein (FP) for wheat lines homozygous for resistant (R) and susceptible (S) alleles at Fhb1, Princeton 2011 (Increase lines included).

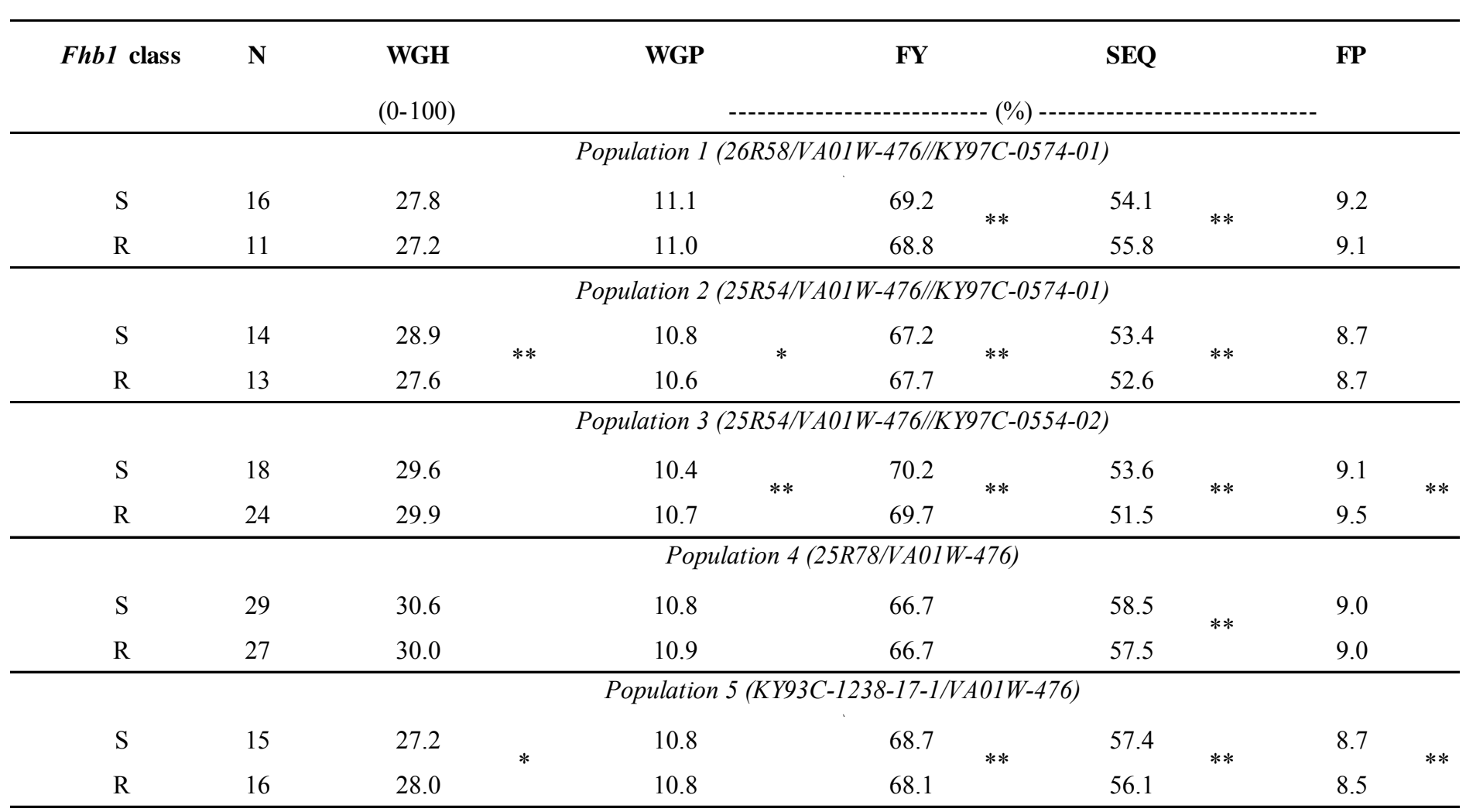

*, ** (Differences between $\mathrm{S}$ and $\mathrm{R}$ classes significant at $\mathrm{P}<0.05,0.01$ ) 
Table A.3.55: Mean gluten performance index (GPI), flour lactic acid solvent retention capacity (LASRC), flour sucrose solvent retention capacity (SSRC), flour water solvent retention capacity (WSRC), flour sodium carbonate solvent retention capacity (SCSRC), wheat meal sedimentation test (SDS) and wheat meal SCSRC (WMSCSRC) for wheat lines homozygous for resistant (R) and susceptible (S) alleles at Fhbl. Princeton 2011 (Increase lines included).

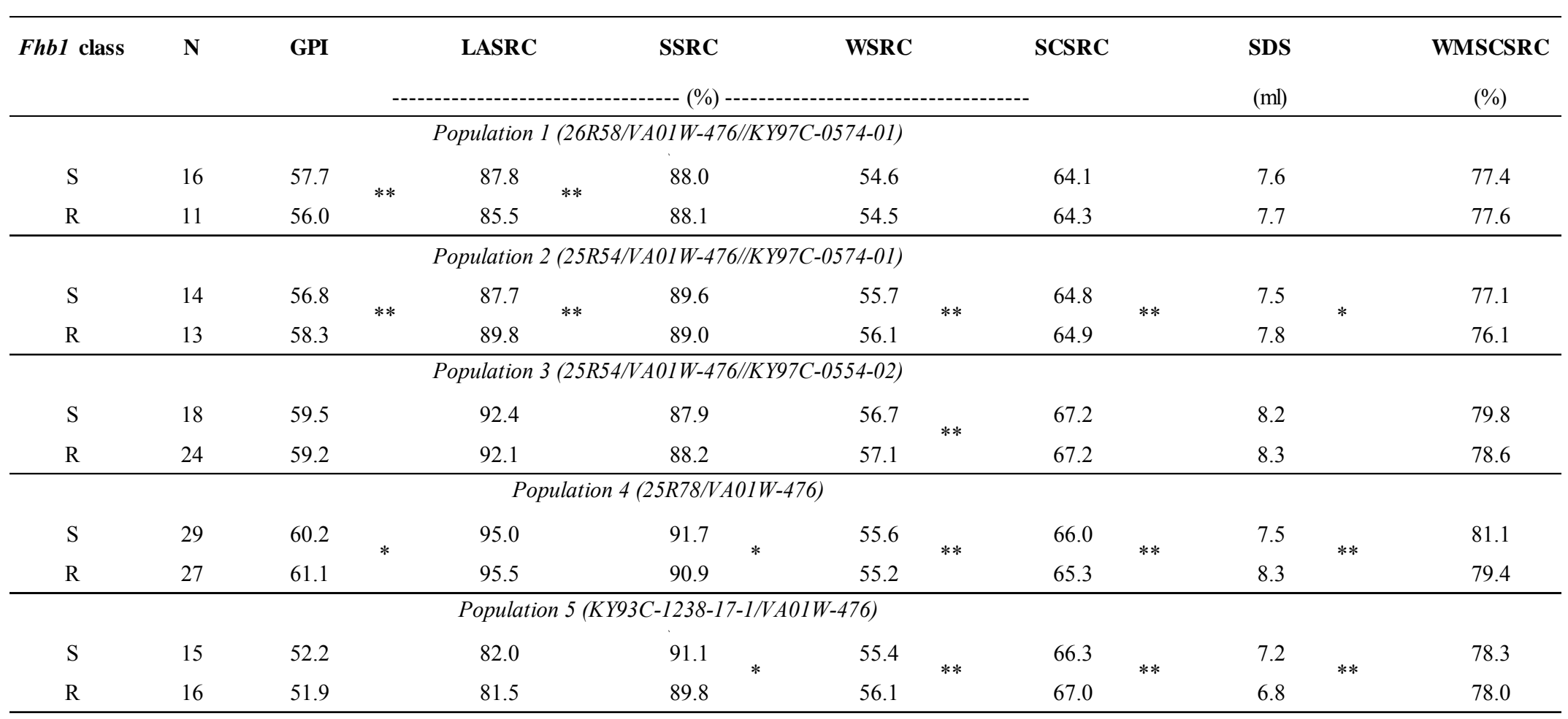

*, ** (Differences between $\mathrm{S}$ and $\mathrm{R}$ classes significant at $\mathrm{P}<0.05,0.01$ ) 
Table A.3.56: Mean Fusarium damaged kernels (FDK), deoxynivalenol level (DON), FHB index, severity, incidence, FHB rating, heading date and plant height for wheat lines homozygous susceptible (S), resistant (R) and heterozygous $(\mathrm{H})$ wheat lines at QFhs.nau-2DL, Lexington 2011 (Increase lines included).

\begin{tabular}{|c|c|c|c|c|c|c|c|c|c|c|c|c|c|c|c|c|c|}
\hline $2 D L$ class & $\mathbf{N}$ & $\begin{array}{c}\text { FDK } \\
(\%)\end{array}$ & & $\begin{array}{l}\text { DON } \\
(\mathrm{ppm})\end{array}$ & & Index & --- & $\begin{array}{c}\text { Severity } \\
---(\%)\end{array}$ & & Incidence & & $\begin{array}{c}\text { Rating } \\
(0-9)\end{array}$ & & $\begin{array}{c}\text { HD } \\
\text { (Julian) }\end{array}$ & & $\begin{array}{c}\text { Height } \\
(\mathrm{cm})\end{array}$ & \\
\hline & & \multicolumn{16}{|c|}{ Population 1 (26R58/VA01W-476//KY97C-0574-01) } \\
\hline S & 13 & 26.6 & a & 26.5 & a & 38.7 & a & 52.7 & a & 71.7 & a & 4.3 & a & 133.3 & a & 81.4 & a \\
\hline $\mathrm{H}$ & 9 & 21.8 & $\mathrm{~b}$ & 22.8 & $a b$ & 33.4 & a & 49.4 & $a b$ & 67.8 & a & 4.2 & a & 132.0 & $\mathrm{~b}$ & 82.6 & a \\
\hline $\mathrm{R}$ & 3 & 19.7 & $\mathrm{~b}$ & 20.4 & $\mathrm{~b}$ & 14.3 & $\mathrm{~b}$ & 38.8 & $\mathrm{~b}$ & 40.0 & $\mathrm{~b}$ & 2.0 & $\mathrm{~b}$ & 133.3 & $\mathrm{ab}$ & 86.4 & a \\
\hline & & \multicolumn{16}{|c|}{ Population $2(25 R 54 / V A 01 W-476 / / K Y 97 C-0574-01)$} \\
\hline $\mathrm{S}$ & 4 & 17.0 & a & 25.8 & a & 29.2 & a & 49.1 & a & 59.4 & a & 3.3 & a & 131.9 & $\mathrm{~b}$ & 87.0 & a \\
\hline $\mathrm{H}$ & 18 & 16.9 & a & 24.4 & $a b$ & 29.7 & a & 43.8 & a & 66.1 & a & 3.5 & a & 135.3 & a & 88.1 & a \\
\hline $\mathrm{R}$ & 4 & 18.2 & a & 21.4 & $\mathrm{~b}$ & 28.4 & a & 40.8 & a & 68.8 & a & 4.0 & a & 131.6 & $\mathrm{~b}$ & 84.5 & a \\
\hline & & \multicolumn{16}{|c|}{ Population $3(25 R 54 / V A 01$ W-476//KY97C-0554-02) } \\
\hline $\mathrm{S}$ & 18 & 19.7 & $\mathrm{~b}$ & 19.5 & a & 33.2 & a & 47.1 & a & 65.3 & a & 4.4 & a & 131.6 & $\mathrm{~b}$ & 84.1 & a \\
\hline $\mathrm{H}$ & 13 & 25.4 & a & 22.8 & $\mathrm{~b}$ & 40.9 & a & 52.9 & a & 72.9 & a & 4.7 & a & 133.7 & a & 80.8 & a \\
\hline $\mathrm{R}$ & 8 & 19.5 & $\mathrm{~b}$ & 21.9 & $\mathrm{ab}$ & 38.6 & a & 51.3 & a & 71.9 & a & 4.2 & a & 133.6 & a & 84.9 & a \\
\hline & & \multicolumn{16}{|c|}{ Population $4(25 R 78 / V A 01 W-476)$} \\
\hline $\mathrm{S}$ & 15 & 31.7 & a & 21.8 & a & 30.9 & a & 53.3 & a & 55.5 & a & 4.3 & a & 130.8 & a & 74.8 & a \\
\hline $\mathrm{H}$ & 26 & 22.4 & $\mathrm{~b}$ & 18.4 & $\mathrm{~b}$ & 31.8 & a & 52.2 & a & 56.4 & a & 4.1 & a & 131.0 & a & 77.4 & a \\
\hline $\mathrm{R}$ & 9 & 23.4 & $\mathrm{~b}$ & 16.8 & $\mathrm{~b}$ & 30.8 & a & 52.5 & a & 57.2 & $\mathrm{a}$ & 3.9 & a & 130.9 & a & 75.4 & a \\
\hline & & \multicolumn{16}{|c|}{ Population 5 (KY93C-1238-17-1/VA01W-476) } \\
\hline $\mathrm{S}$ & 6 & 14.1 & $\mathrm{~b}$ & 18.6 & a & 33.2 & a & 58.0 & a & 55.8 & $\mathrm{ab}$ & 4.1 & $\mathrm{ab}$ & 131.2 & a & 84.7 & a \\
\hline $\mathrm{H}$ & 15 & 18.7 & a & 18.7 & $\mathrm{a}$ & 35.7 & a & 51.0 & $a b$ & 68.5 & a & 4.2 & a & 131.5 & a & 86.3 & a \\
\hline $\mathrm{R}$ & 10 & 13.6 & $\mathrm{~b}$ & 13.2 & $\mathrm{~b}$ & 28.4 & $\mathrm{a}$ & 47.7 & $\mathrm{~b}$ & 56.5 & $\mathrm{~b}$ & 3.3 & $\mathrm{~b}$ & 131.0 & a & 79.6 & $\mathrm{~b}$ \\
\hline
\end{tabular}

Means (by population) within the same column followed by different letters are significantly different at $\mathrm{P}<0.05$ 
1 Table A.3.57: Mean yield, test weight (TWT), plant height, heading date (HD) and FHB rating measured in plots for homozygous susceptible (S), resistant $(\mathrm{R})$ and heterozygous (H) wheat lines at QFhs.nau-2DL, Lexington 2011 (Increase lines included).

\begin{tabular}{|c|c|c|c|c|c|c|c|c|c|c|c|}
\hline $2 D L$ class & $\mathbf{N}$ & $\begin{array}{c}\text { Yield } \\
(\mathrm{kg} / \mathrm{ha})\end{array}$ & & $\begin{array}{l}\text { TWT } \\
(\mathrm{kg} / \mathrm{hl})\end{array}$ & & $\begin{array}{c}\text { Height } \\
(\mathrm{cm})\end{array}$ & & $\begin{array}{c}\text { HD } \\
\text { (Julian) }\end{array}$ & & $\begin{array}{c}\text { Rating } \\
(0-9)\end{array}$ & \\
\hline & \multicolumn{11}{|c|}{ Population 1 (26R58/VA01W-476//KY97C-0574-01) } \\
\hline S & 13 & 3799 & a & 67.3 & $\mathrm{~b}$ & 81.6 & c & 130.9 & $\mathrm{ab}$ & 3.8 & $\mathrm{a}$ \\
\hline $\mathrm{H}$ & 9 & 3915 & $\mathrm{a}$ & 69.4 & $\mathrm{a}$ & 84.5 & $\mathrm{~b}$ & 130.5 & $\mathrm{~b}$ & 3.4 & $\mathrm{ab}$ \\
\hline \multirow[t]{2}{*}{$\mathrm{R}$} & 3 & 3685 & $\mathrm{a}$ & 70.3 & $\mathrm{a}$ & 91.7 & a & 131.3 & $\mathrm{a}$ & 2.7 & $\mathrm{~b}$ \\
\hline & \multicolumn{11}{|c|}{ Population 2 (25R54/VA01W-476//KY97C-0574-01) } \\
\hline S & 4 & 3705 & a & 69.4 & $\mathrm{c}$ & 83.6 & $\mathrm{~b}$ & 131.2 & $\mathrm{~b}$ & 3.3 & a \\
\hline $\mathrm{H}$ & 18 & 3839 & $\mathrm{a}$ & 71.8 & $\mathrm{~b}$ & 90.7 & $\mathrm{a}$ & 133.5 & $\mathrm{a}$ & 2.0 & $\mathrm{~b}$ \\
\hline \multirow[t]{2}{*}{$\mathrm{R}$} & 4 & 3945 & $\mathrm{a}$ & 72.4 & $\mathrm{a}$ & 87.6 & $a b$ & 129.9 & c & 3.5 & $\mathrm{a}$ \\
\hline & \multicolumn{11}{|c|}{ Population $3(25 R 54 / V A 01$ W-476//KY97C-0554-02) } \\
\hline S & 18 & 4126 & $\mathrm{a}$ & 70.5 & $\mathrm{a}$ & 81.9 & a & 130.3 & $\mathrm{c}$ & 3.5 & a \\
\hline $\mathrm{H}$ & 13 & 3574 & $\mathrm{c}$ & 67.7 & $\mathrm{c}$ & 78.8 & $\mathrm{~b}$ & 131.5 & $\mathrm{~b}$ & 3.7 & a \\
\hline \multirow[t]{2}{*}{$\mathrm{R}$} & 8 & 3803 & $\mathrm{~b}$ & 69.3 & $\mathrm{~b}$ & 82.4 & a & 132.0 & $\mathrm{a}$ & 3.0 & $\mathrm{~b}$ \\
\hline & \multicolumn{11}{|c|}{ Population $4(25 R 78 / V A 01 W-476)$} \\
\hline S & 15 & 3248 & $\mathrm{~b}$ & 67.3 & $\mathrm{~b}$ & 73.7 & $\mathrm{~b}$ & 129.9 & $\mathrm{a}$ & 2.4 & a \\
\hline $\mathrm{H}$ & 26 & 3456 & $\mathrm{a}$ & 68.6 & $\mathrm{a}$ & 74.9 & $\mathrm{~b}$ & 129.9 & a & 2.0 & $\mathrm{~b}$ \\
\hline \multirow[t]{2}{*}{$\mathrm{R}$} & 9 & 3554 & $\mathrm{a}$ & 69.0 & $\mathrm{a}$ & 78.5 & $\mathrm{a}$ & 130.2 & $\mathrm{a}$ & 1.9 & $\mathrm{~b}$ \\
\hline & \multicolumn{11}{|c|}{ Population 5 (KY93C-1238-17-1/VA01W-476) } \\
\hline S & 6 & 4079 & a & 70.9 & a & 83.4 & a & 130.2 & $\mathrm{ab}$ & 2.7 & $\mathrm{~b}$ \\
\hline $\mathrm{H}$ & 15 & 4019 & $\mathrm{a}$ & 70.1 & $\mathrm{a}$ & 83.0 & $\mathrm{a}$ & 130.4 & $\mathrm{a}$ & 3.4 & $\mathrm{a}$ \\
\hline $\mathrm{R}$ & 10 & 4049 & $\mathrm{a}$ & 70.3 & $\mathrm{a}$ & 80.1 & $\mathrm{~b}$ & 129.5 & $\mathrm{~b}$ & 2.9 & $\mathrm{~b}$ \\
\hline
\end{tabular}

3 Means (by population) within the same column followed by different letters are significantly different at $\mathrm{P}<0.05$ 
Table A.3.58: Mean yield, test weight (TWT), plant height, heading date (HD) and FHB

5 rating measured in plots for homozygous susceptible $(\mathrm{S})$, resistant $(\mathrm{R})$ and heterozygous (H) wheat lines at QFhs.nau-2DL, Princeton 2011 (Increase lines included).

\begin{tabular}{|c|c|c|c|c|c|c|c|c|c|c|c|}
\hline $2 D L$ class & $\mathbf{N}$ & $\begin{array}{c}\text { Yield } \\
(\mathrm{kg} / \mathrm{ha})\end{array}$ & & $\begin{array}{c}\text { TWT } \\
(\mathrm{kg} / \mathrm{hl})\end{array}$ & & $\begin{array}{c}\text { Height } \\
\text { (cm) }\end{array}$ & & $\begin{array}{c}\text { HD } \\
\text { (Julian) }\end{array}$ & & $\begin{array}{c}\text { Rating } \\
(0-9)\end{array}$ & \\
\hline & \multicolumn{11}{|c|}{ Population 1 (26R58/VA01W-476//KY97C-0574-01) } \\
\hline $\mathrm{S}$ & 13 & 4229 & a & 70.1 & $\mathrm{~b}$ & 90.1 & $\mathrm{a}$ & 122.2 & $\mathrm{a}$ & 2.6 & $\mathrm{a}$ \\
\hline $\mathrm{H}$ & 9 & 4559 & $\mathrm{~b}$ & 71.1 & $\mathrm{a}$ & 89.9 & $\mathrm{a}$ & 121.2 & $\mathrm{~b}$ & 2.4 & $\mathrm{a}$ \\
\hline \multirow[t]{2}{*}{$\mathrm{R}$} & 3 & 4357 & $a b$ & 71.9 & $\mathrm{a}$ & 94.5 & $\mathrm{a}$ & 123.1 & $\mathrm{a}$ & 2.1 & $\mathrm{a}$ \\
\hline & \multicolumn{11}{|c|}{ Population $2(25 R 54 / V A 01 W-476 / / K Y 97 C-0574-01)$} \\
\hline $\mathrm{S}$ & 4 & 4293 & $\mathrm{a}$ & 71.3 & $\mathrm{~b}$ & 92.3 & $\mathrm{~b}$ & 123.2 & $\mathrm{a}$ & 2.7 & $\mathrm{~b}$ \\
\hline $\mathrm{H}$ & 18 & 4356 & $\mathrm{a}$ & 72.4 & $\mathrm{a}$ & 96.7 & $\mathrm{a}$ & 123.9 & $\mathrm{a}$ & 2.0 & $\mathrm{~b}$ \\
\hline \multirow[t]{2}{*}{$\mathrm{R}$} & 4 & 4356 & $\mathrm{a}$ & 72.5 & a & 89.5 & $\mathrm{~b}$ & 120.8 & $\mathrm{~b}$ & 3.3 & $\mathrm{a}$ \\
\hline & \multicolumn{11}{|c|}{ Population 3 (25R54/VA01W-476//KY97C-0554-02) } \\
\hline $\mathrm{S}$ & 18 & 4540 & $\mathrm{a}$ & 72.5 & $\mathrm{a}$ & 86.9 & $\mathrm{a}$ & 121.3 & b & 3.6 & a \\
\hline $\mathrm{H}$ & 13 & 4100 & $\mathrm{~b}$ & 69.9 & $\mathrm{c}$ & 84.3 & $\mathrm{~b}$ & 123.3 & a & 3.7 & a \\
\hline \multirow[t]{2}{*}{$\mathrm{R}$} & 8 & 4213 & $\mathrm{~b}$ & 71.6 & b & 86.5 & $a b$ & 123.5 & $\mathrm{a}$ & 3.0 & $\mathrm{~b}$ \\
\hline & \multicolumn{11}{|c|}{ Population $4(25 R 78 / V A 01 W-476)$} \\
\hline $\mathrm{S}$ & 15 & 3753 & $\mathrm{a}$ & 69.7 & b & 80.2 & $\mathrm{~b}$ & 122.1 & $\mathrm{a}$ & 0.9 & a \\
\hline $\mathrm{H}$ & 26 & 3897 & $\mathrm{a}$ & 71.1 & $\mathrm{a}$ & 80.9 & b & 121.5 & $\mathrm{a}$ & 0.6 & $\mathrm{~b}$ \\
\hline \multirow[t]{2}{*}{$\mathrm{R}$} & 9 & 3786 & $\mathrm{a}$ & 70.7 & $\mathrm{a}$ & 84.6 & $\mathrm{a}$ & 122.0 & $\mathrm{a}$ & 0.9 & $\mathrm{ab}$ \\
\hline & \multicolumn{11}{|c|}{ Population 5 (KY93C-1238-17-1/VA01W-476) } \\
\hline S & 6 & 4440 & $\mathrm{a}$ & 73.1 & a & 96.1 & $\mathrm{a}$ & 122.1 & $\mathrm{a}$ & 1.2 & $\mathrm{ab}$ \\
\hline $\mathrm{H}$ & 15 & 4366 & $\mathrm{a}$ & 72.9 & $\mathrm{a}$ & 95.0 & $\mathrm{a}$ & 121.8 & $\mathrm{a}$ & 1.6 & $\mathrm{a}$ \\
\hline $\mathrm{R}$ & 10 & 4365 & $\mathrm{a}$ & 72.6 & $\mathrm{a}$ & 93.0 & $\mathrm{a}$ & 120.8 & $\mathrm{~b}$ & 0.9 & $\mathrm{~b}$ \\
\hline
\end{tabular}

Means (by population) within the same column followed by different letters are significantly different at $\mathrm{P}<0.05$ 
Table A.3.59: Mean whole grain hardness (WGH), whole grain protein (WGP), flour yield (FY), softness equivalent (SEQ) and flour protein (FP) for homozygous susceptible (S), resistant (R) and heterozygous (H) wheat lines at QFhs.nau-2DL, Princeton 2011 (Increase lines included).

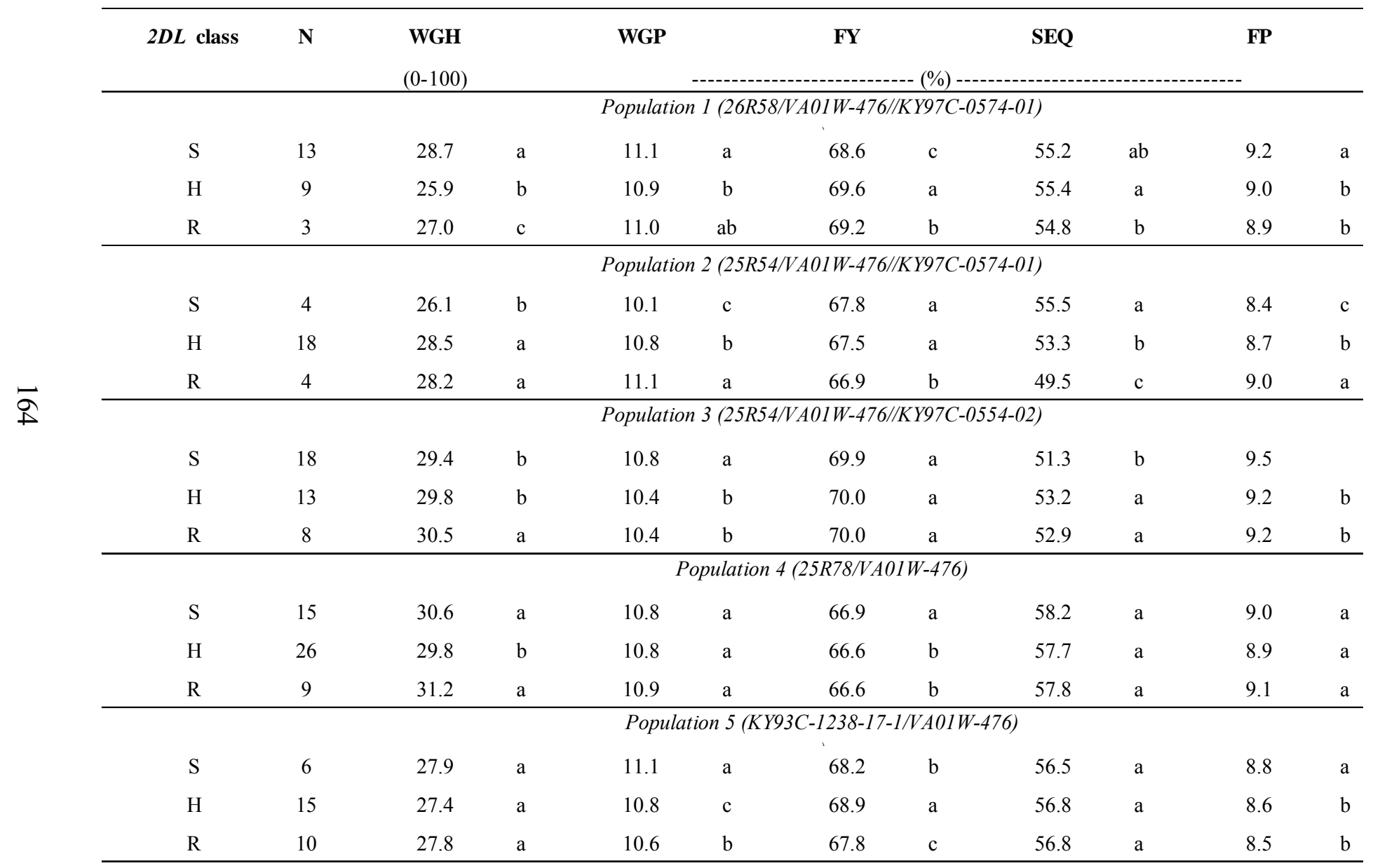

Means (by population) within the same column followed by different letters are significantly different at $\mathrm{P}<0.05$ 
Table A.3.60: Mean gluten performance index (GPI), flour lactic acid solvent retention capacity (LASRC),flour sucrose solvent retention capacity (SSRC), flour water solvent retention capacity (WSRC), sodium carbonate solvent retention capacity (SCSRC), wheat meal sedimentation test (SDS) and wheat meal SCSRC (WMSCSRC) for homozygous susceptible (S), resistant (R) and heterozygous (H) wheat lines at QFhs.nau-2DL, Princeton 2011 (Increase lines included).

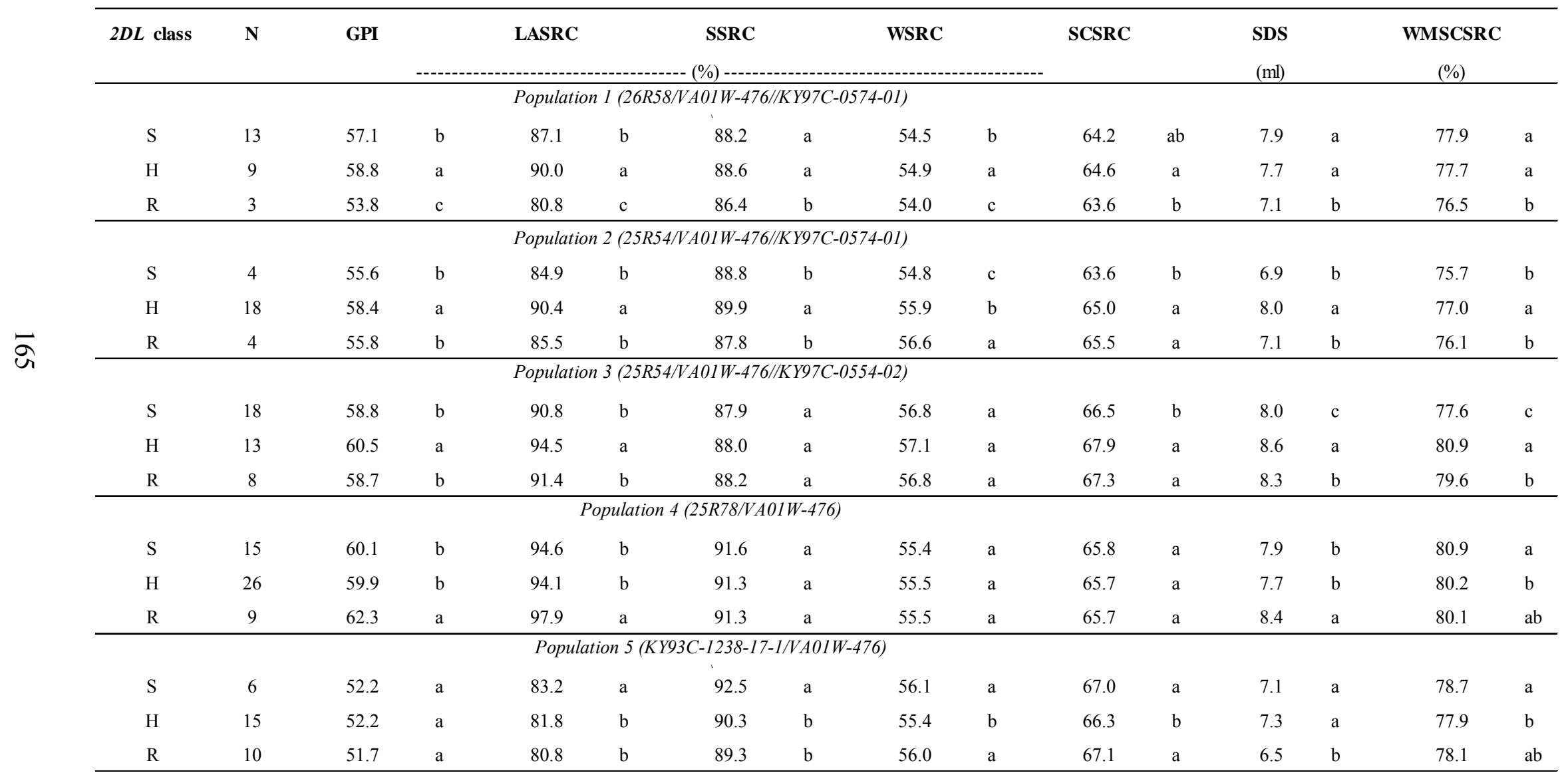

Means (by population) within the same column followed by different letters are significantly different at $\mathrm{P}<0.05$ 
Figure A.3.1: Precipitation and mean temperature during wheat growth season in Lexington 2010. Black bars and empty bars correspond 2010 and historic precipitation levels, respectively.

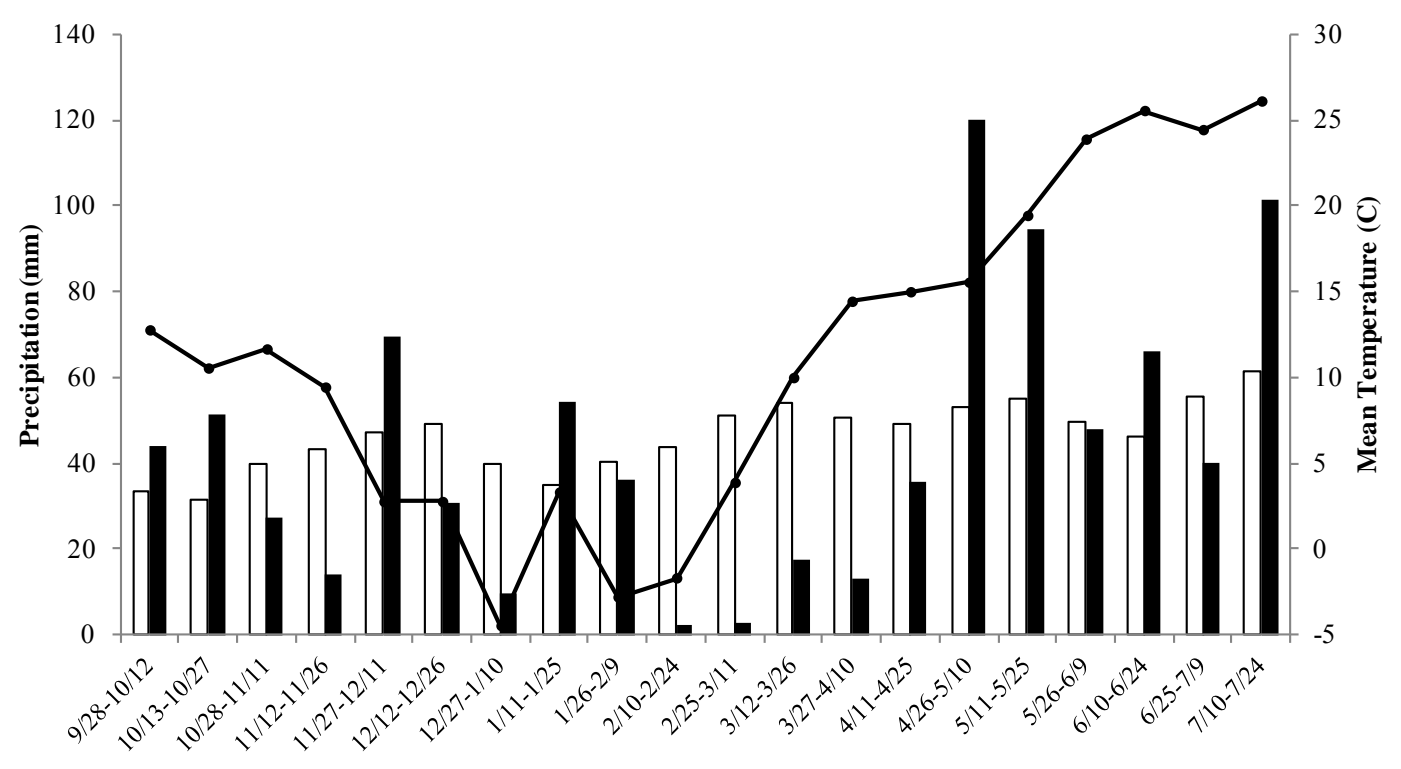


Figure A.3.2: Precipitation and mean temperature during wheat growth season in Lexington 2011. Black bars and empty bars correspond 2011 and historic precipitation levels, respectively.

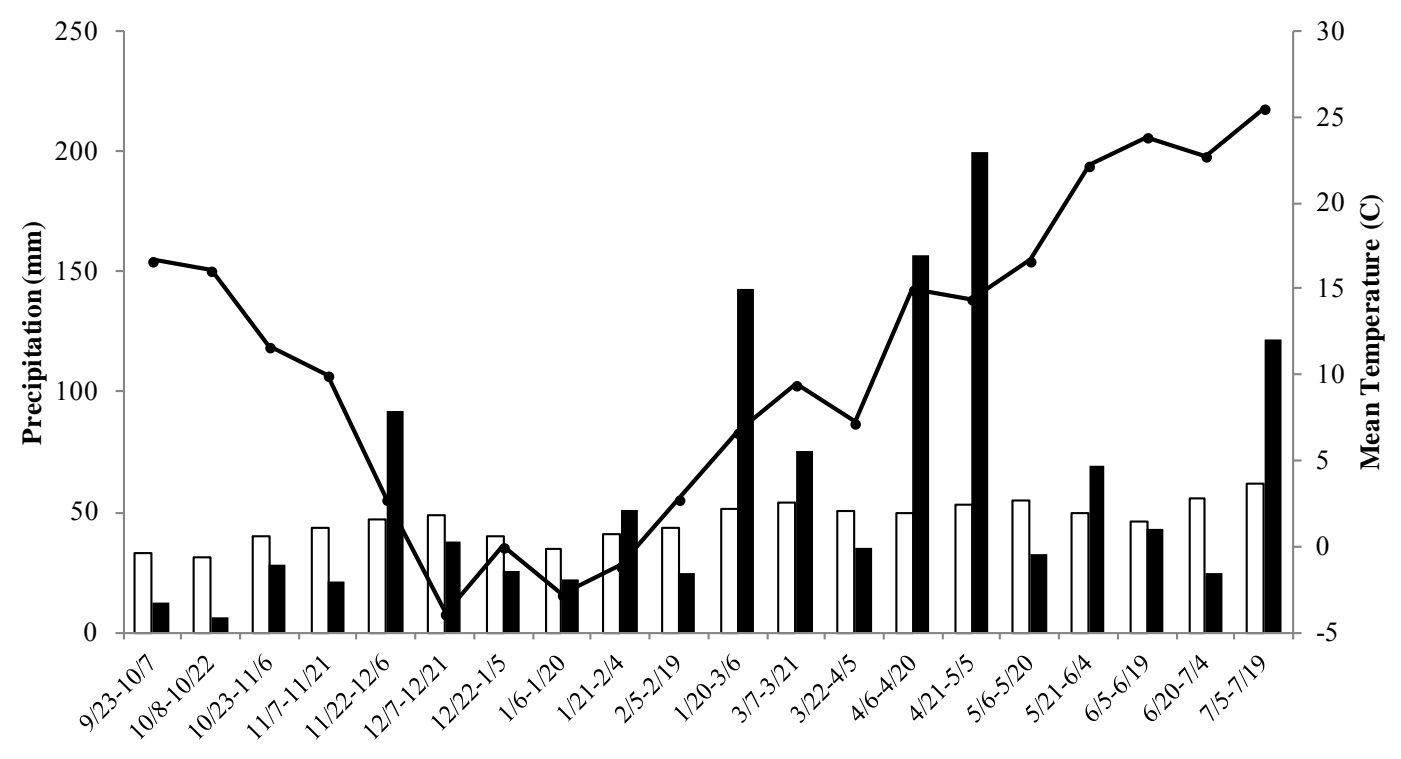


Figure A.3.3: Precipitation and mean temperature during wheat growth season in Princeton 2011. Black bars and empty bars correspond 2011 and historic precipitation levels, respectively.

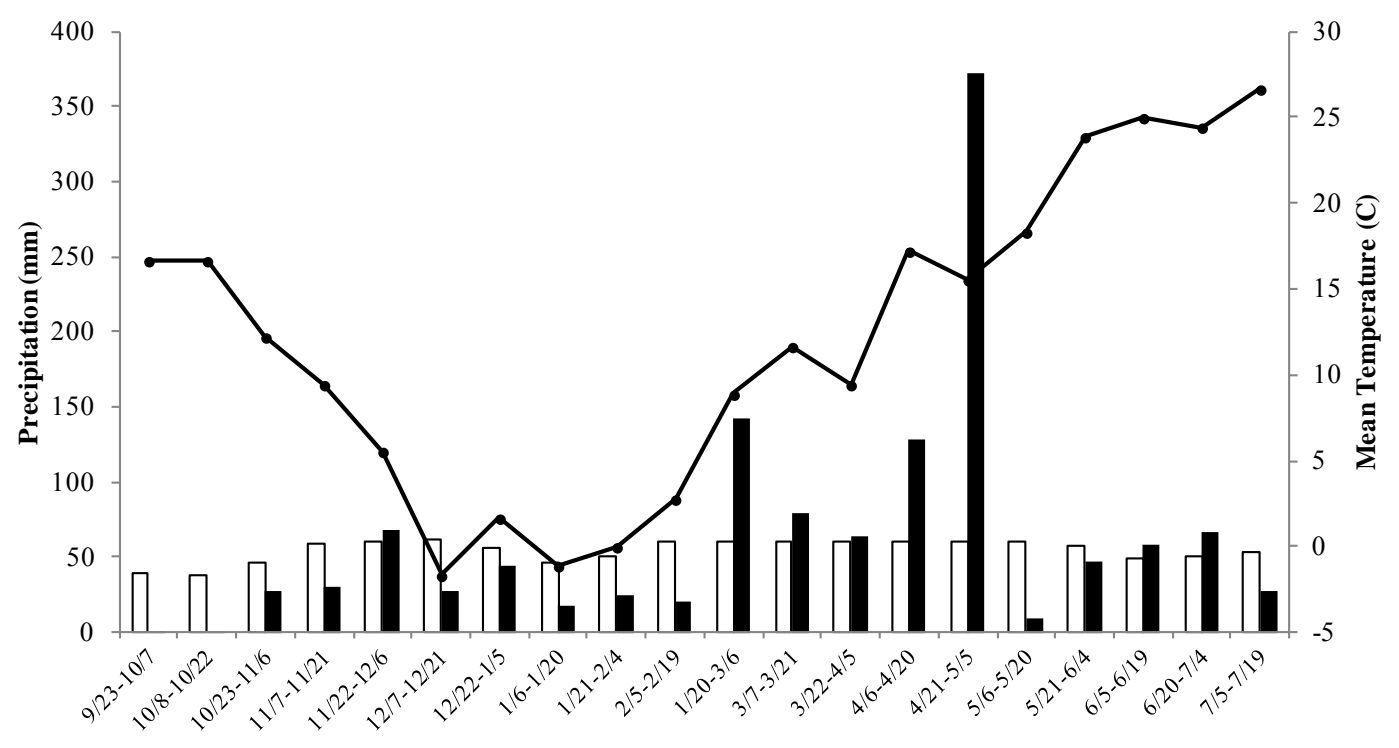


Figure A.3.4: Regression of deoxynivalenol level (DON) on percentage of

Fusarium damaged kernels (FDK) for all populations in Lexington 2010 and 2011.

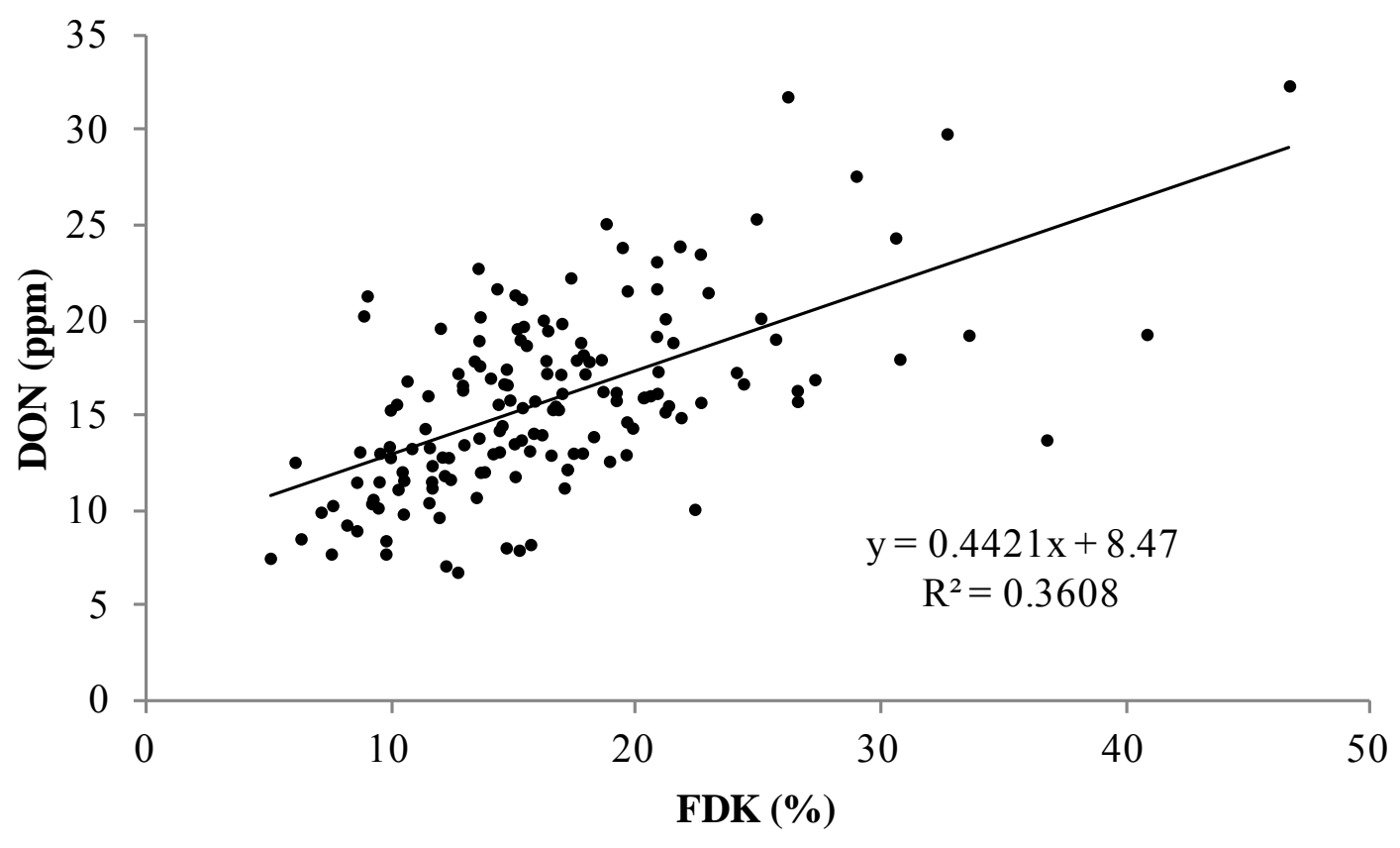


Figure A.3.5: Regression of deoxynivalenol level (DON) on percentage of Fusarium damaged kernels (FDK) in population 1 in Lexington 2010 and 2011.

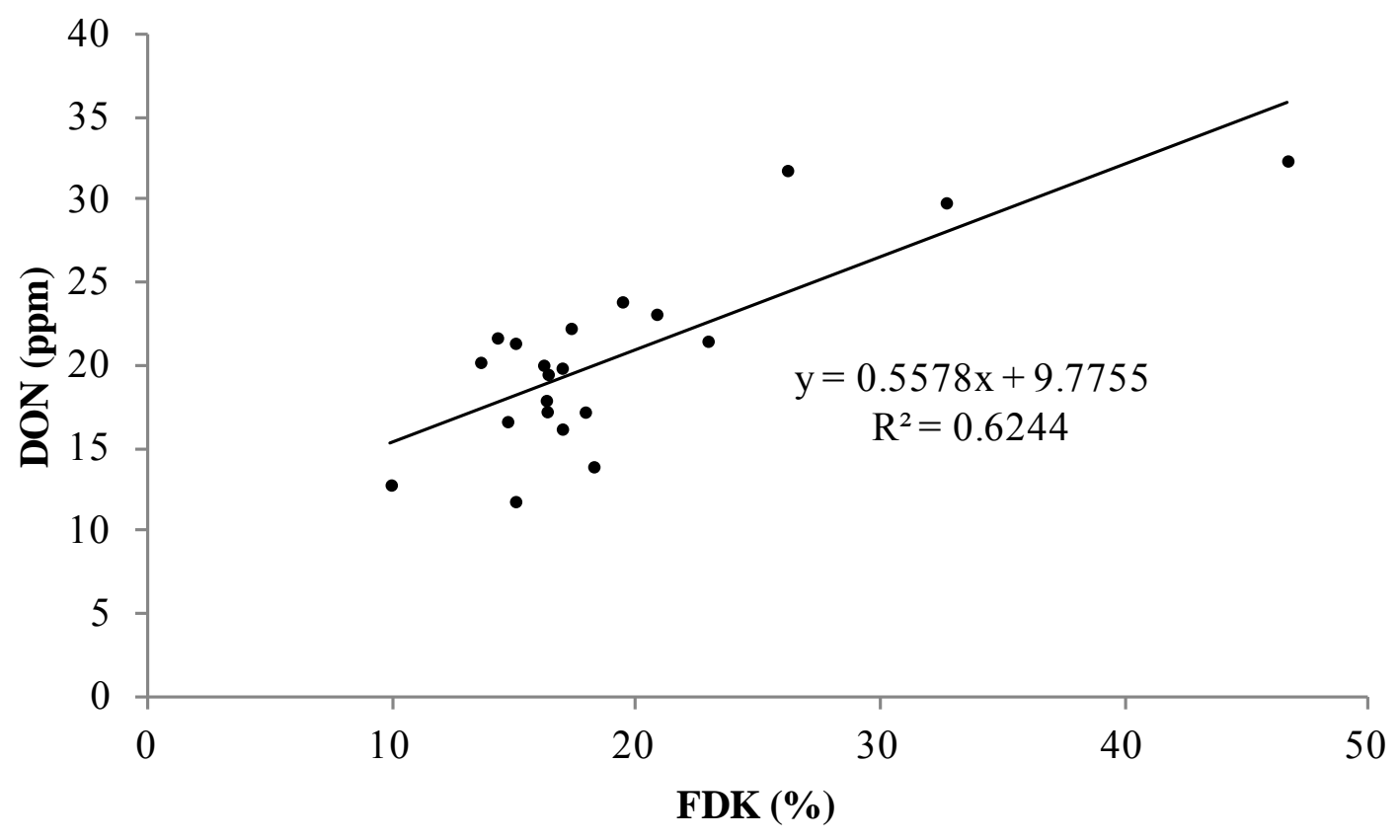


Figure A.3.6: Regression of deoxynivalenol level (DON) on percentage of Fusarium damaged kernels (FDK) in population 2 in Lexington 2010 and 2011.

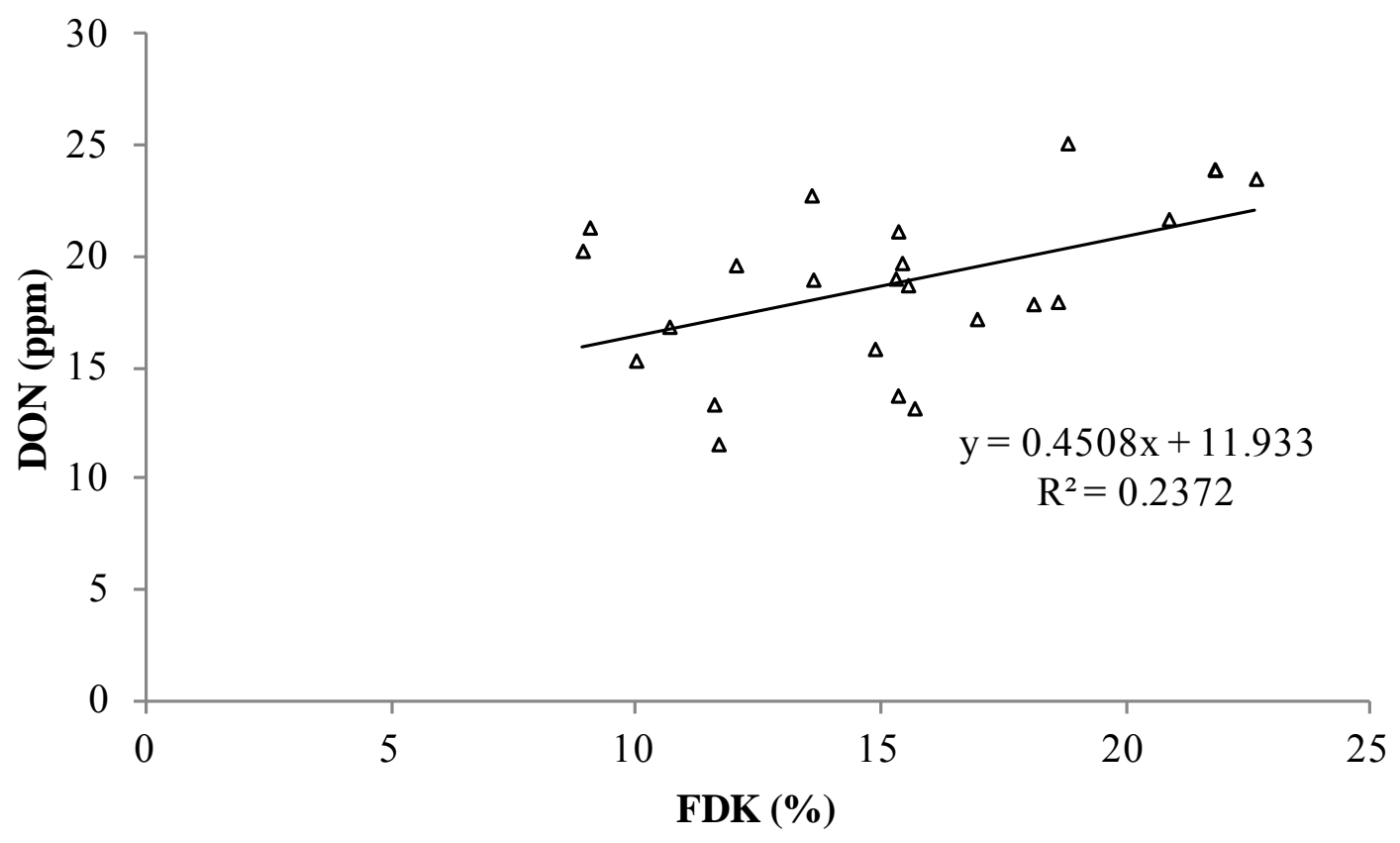


Figure A.3.7: Regression of deoxynivalenol level (DON) on percentage of Fusarium damaged kernels (FDK) in population 3 in Lexington 2010 and 2011.

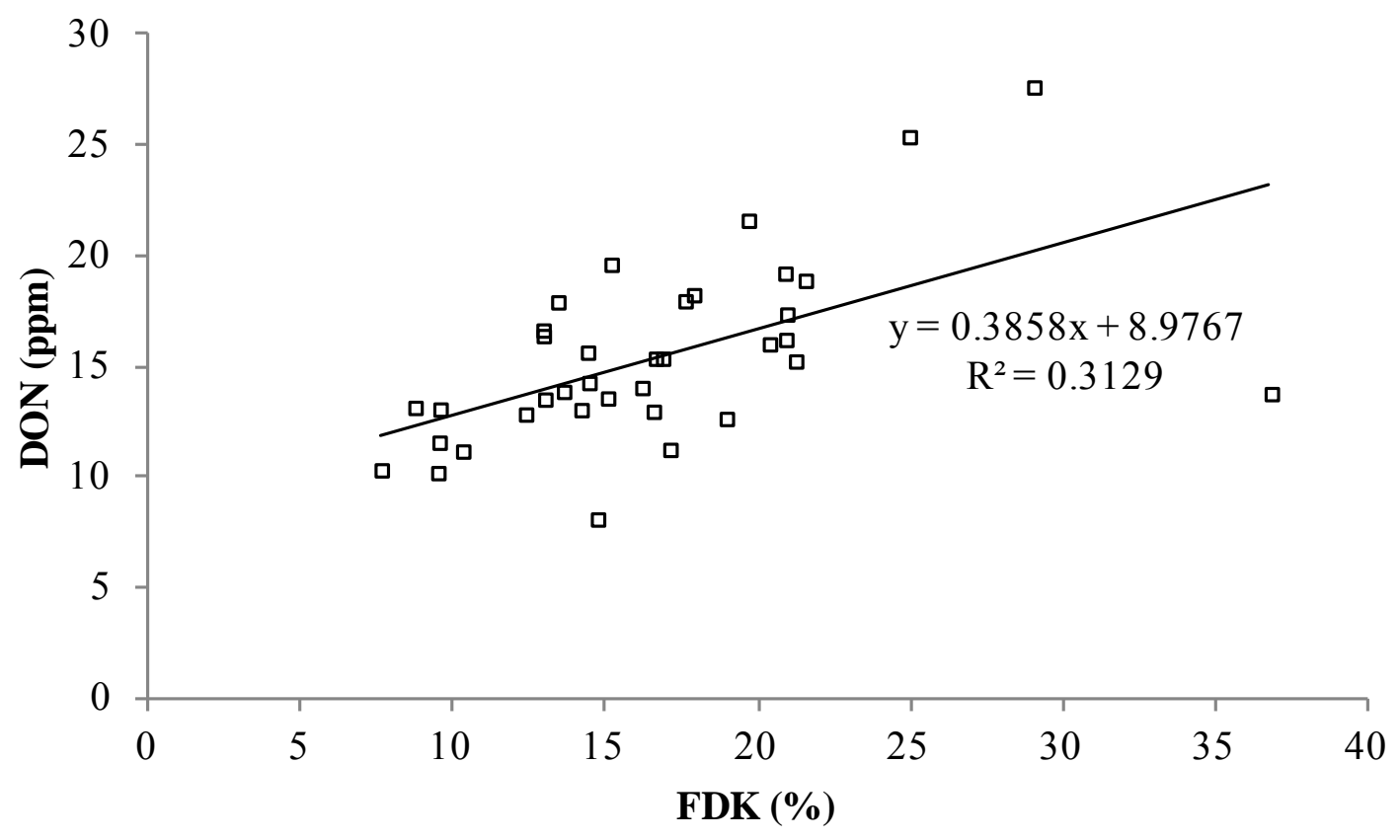


Figure A.3.8: Regression of deoxynivalenol level (DON) on percentage of Fusarium damaged kernels (FDK) in population 4 in Lexington 2010 and 2011.

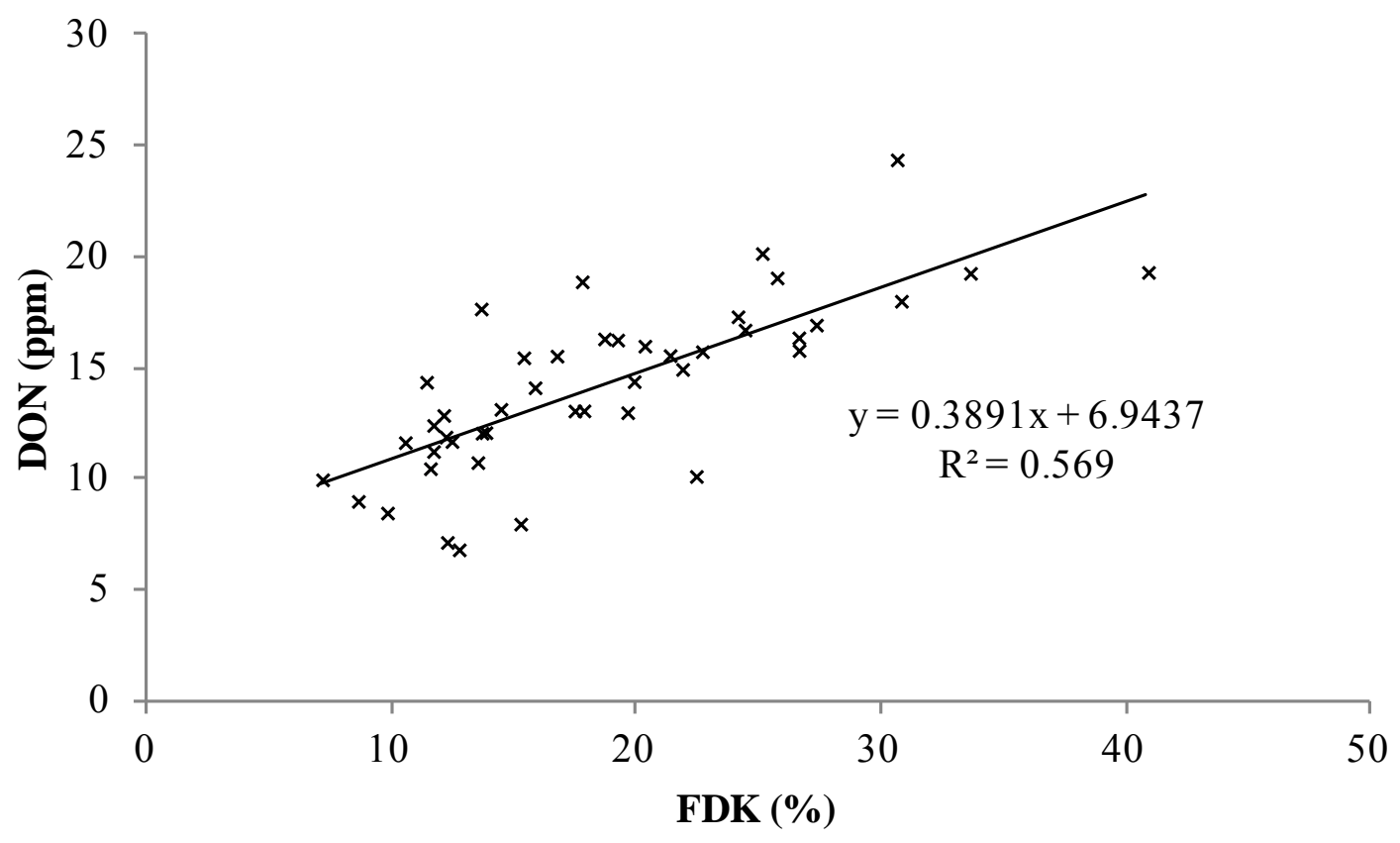


Figure A.3.9: Regression of deoyinivalenol level (DON) on percentage of Fusarium damaged kernels (FDK) in population 5 in Lexington 2010 and 2011.

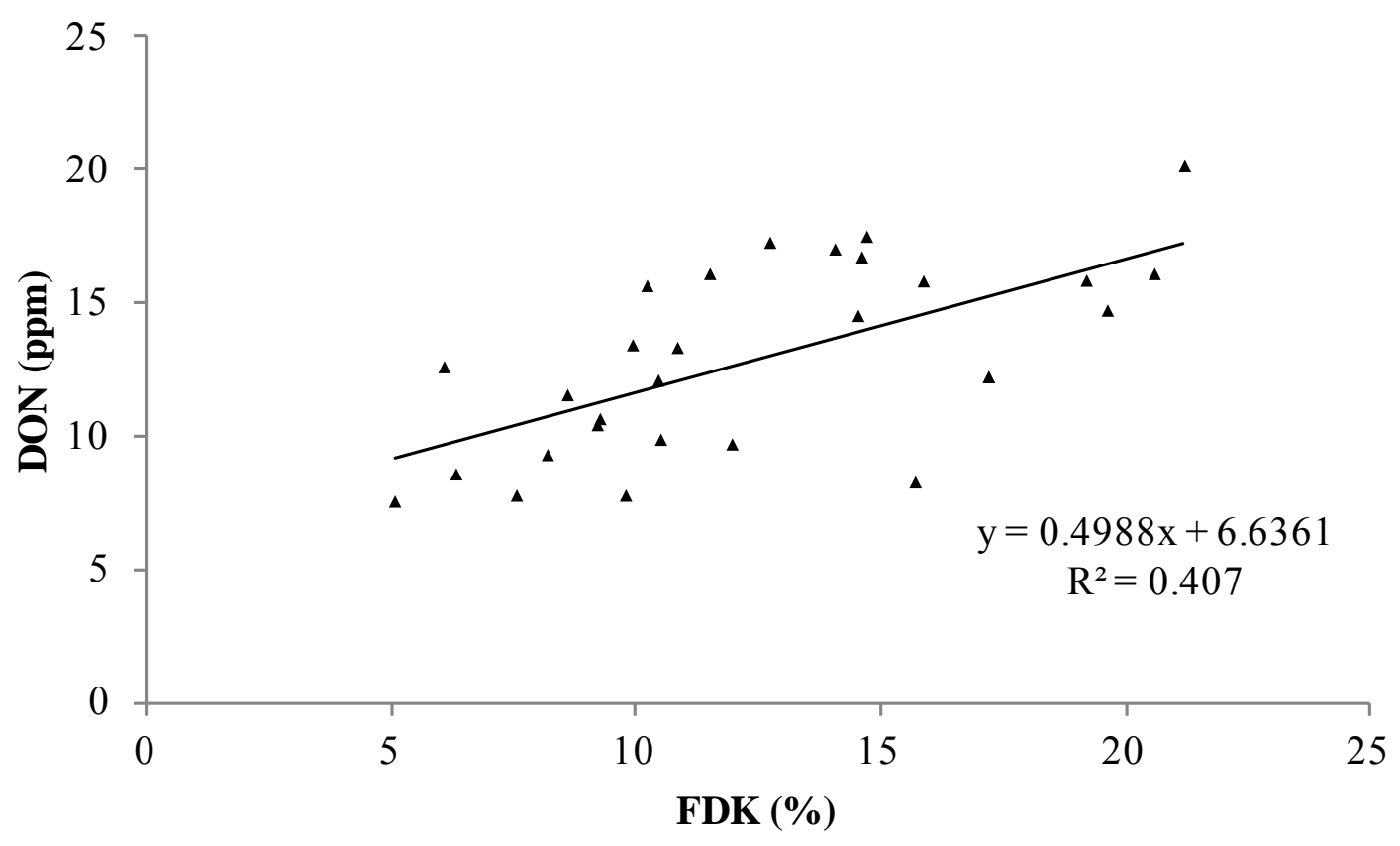


Figure A.3.10: Regression of deoxynivalenol level (DON) on Fusarium damaged kernels (FDK) for all populations in 2010 and 2011.

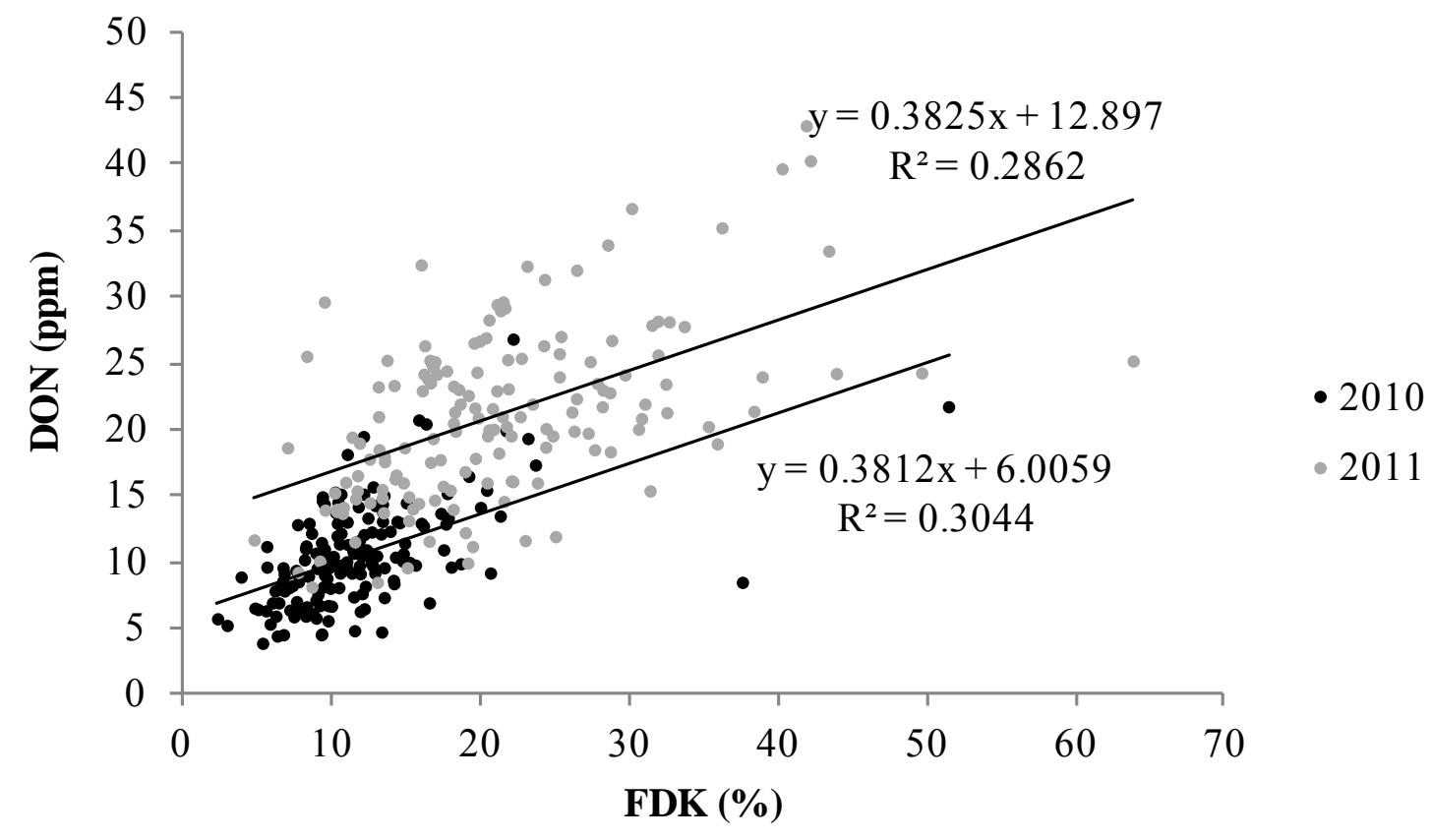


Figure A.3.11: Regression of deoxynivalenol level (DON) on Fusarium damaged kernels (FDK) for populations 1 in Lexington 2010 and 2011.

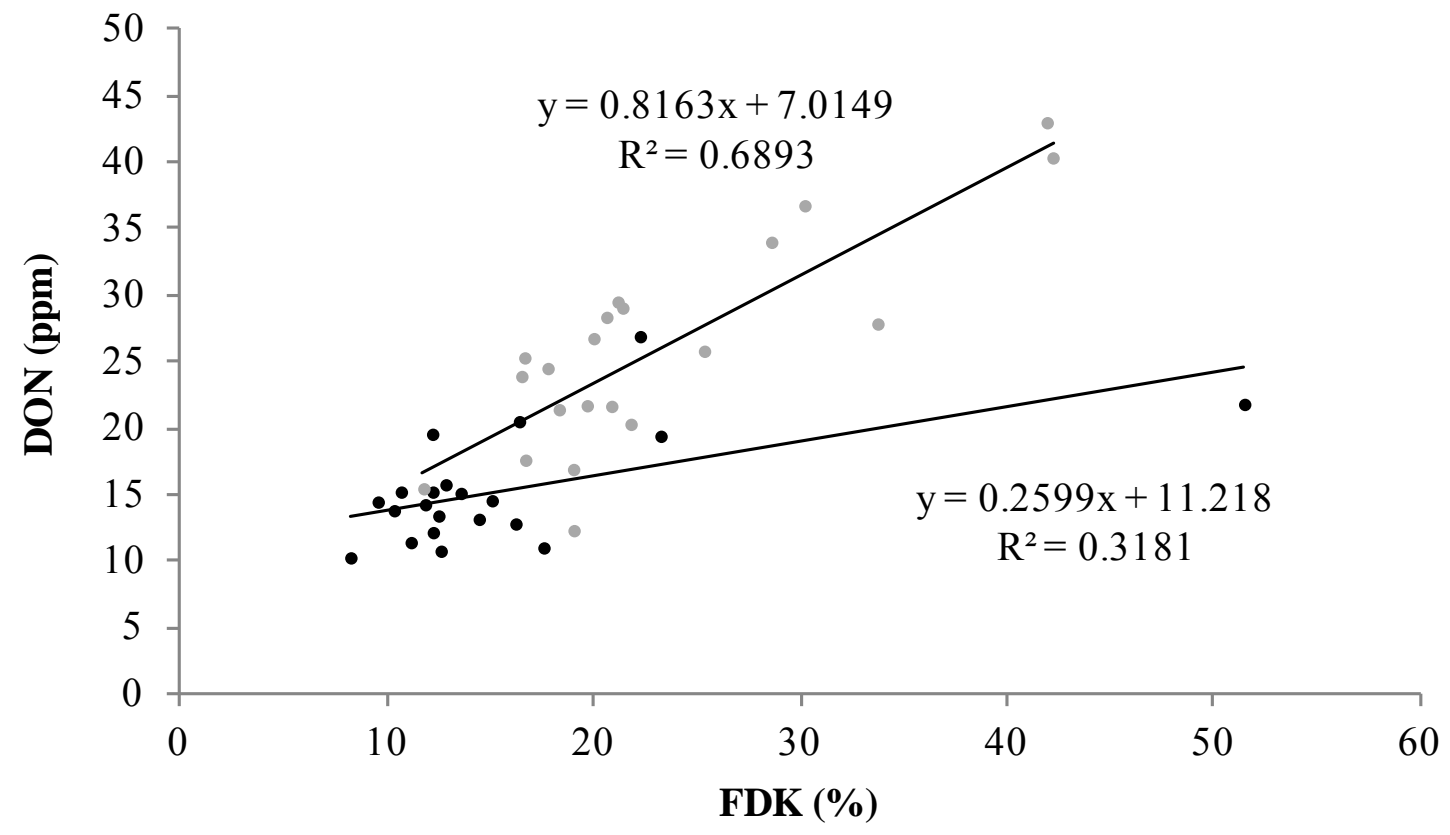


Figure A.3.12: Regression of deoxynivalenol level (DON) on Fusarium damaged kernels (FDK) for populations 2 in Lexington 2010 and 2011.

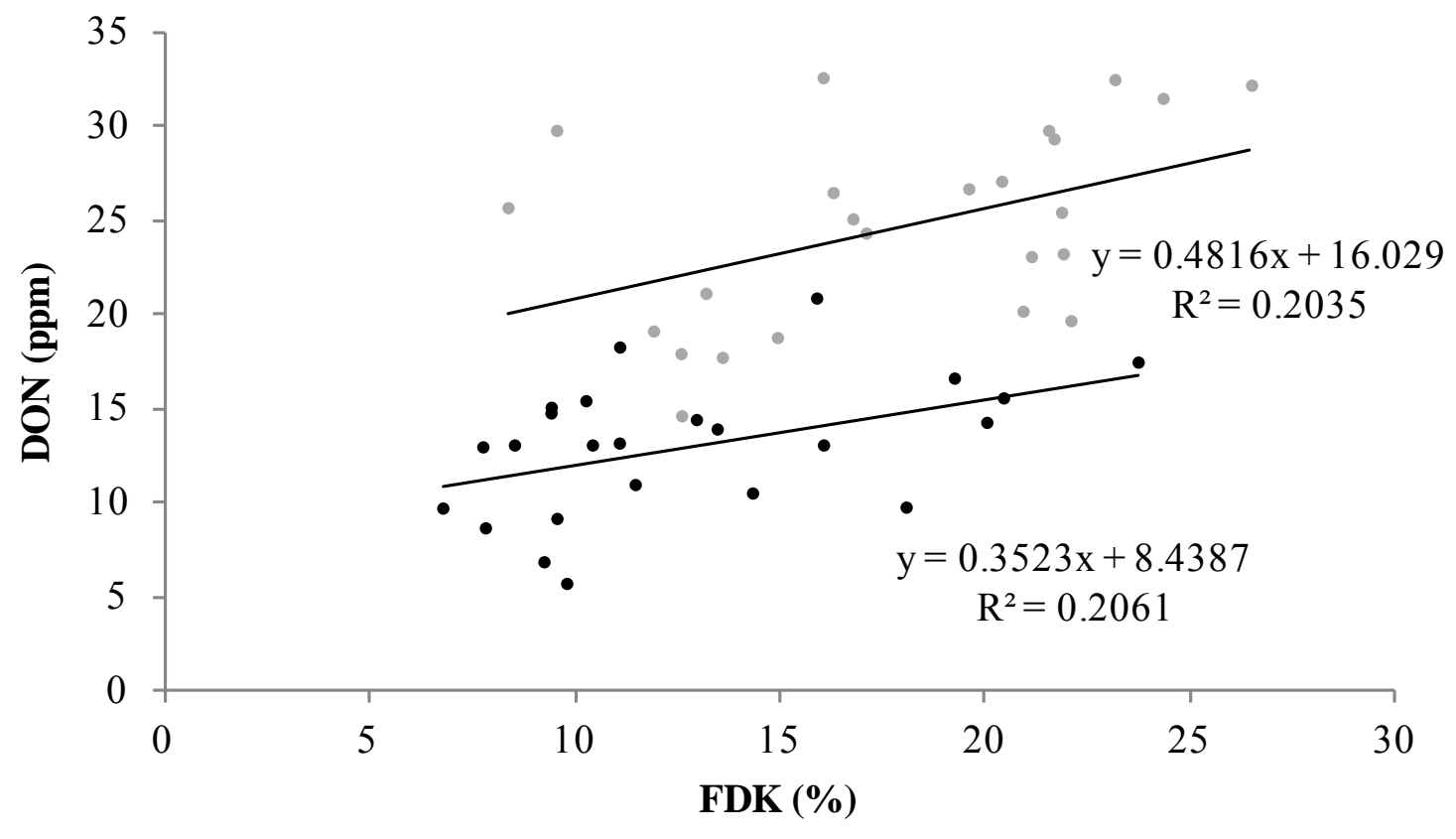


Figure A.3.13: Regression of deoxynivalenol level (DON) on Fusarium damaged kernels (FDK) for populations 3 in Lexington 2010 and 2011.

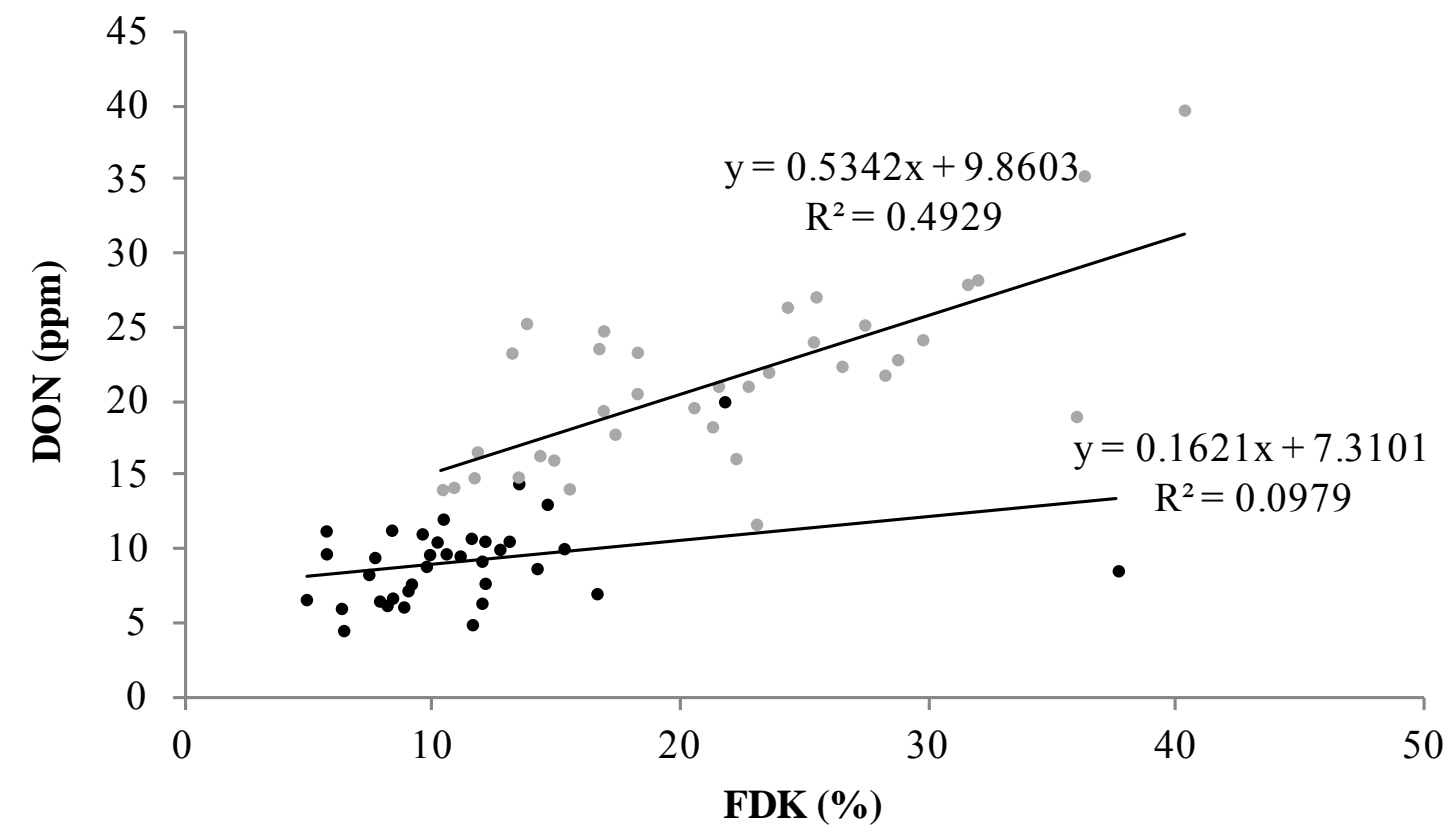


Figure A.3.14: Regression of deoxynivalenol level (DON) on Fusarium damaged kernels (FDK) for populations 4 in Lexington 2010 and 2011.

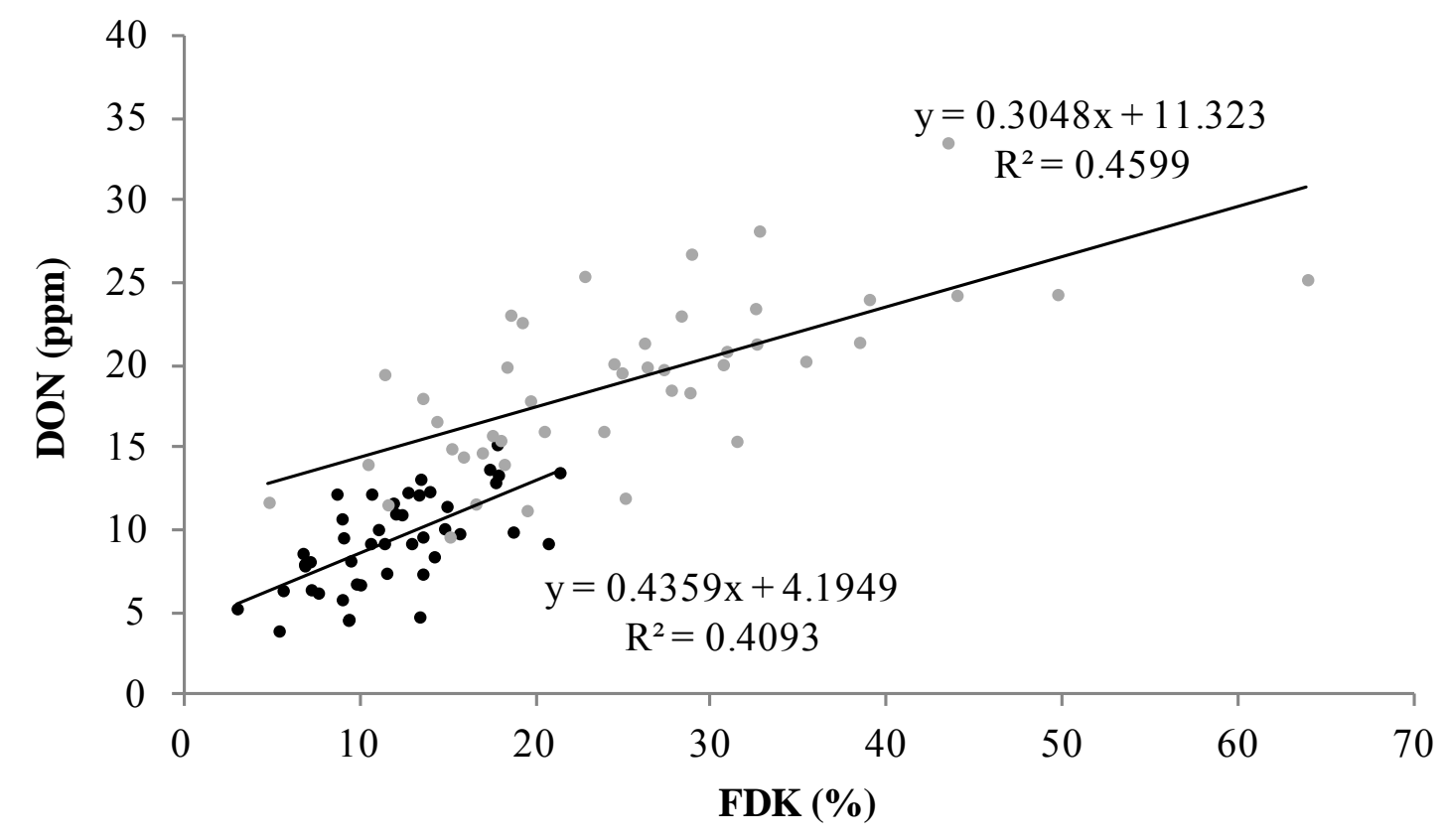


Figure A.3.15: Regression of deoxynivalenol level (DON) on Fusarium damaged kernels (FDK) for populations 5 in Lexington 2010 and 2011.

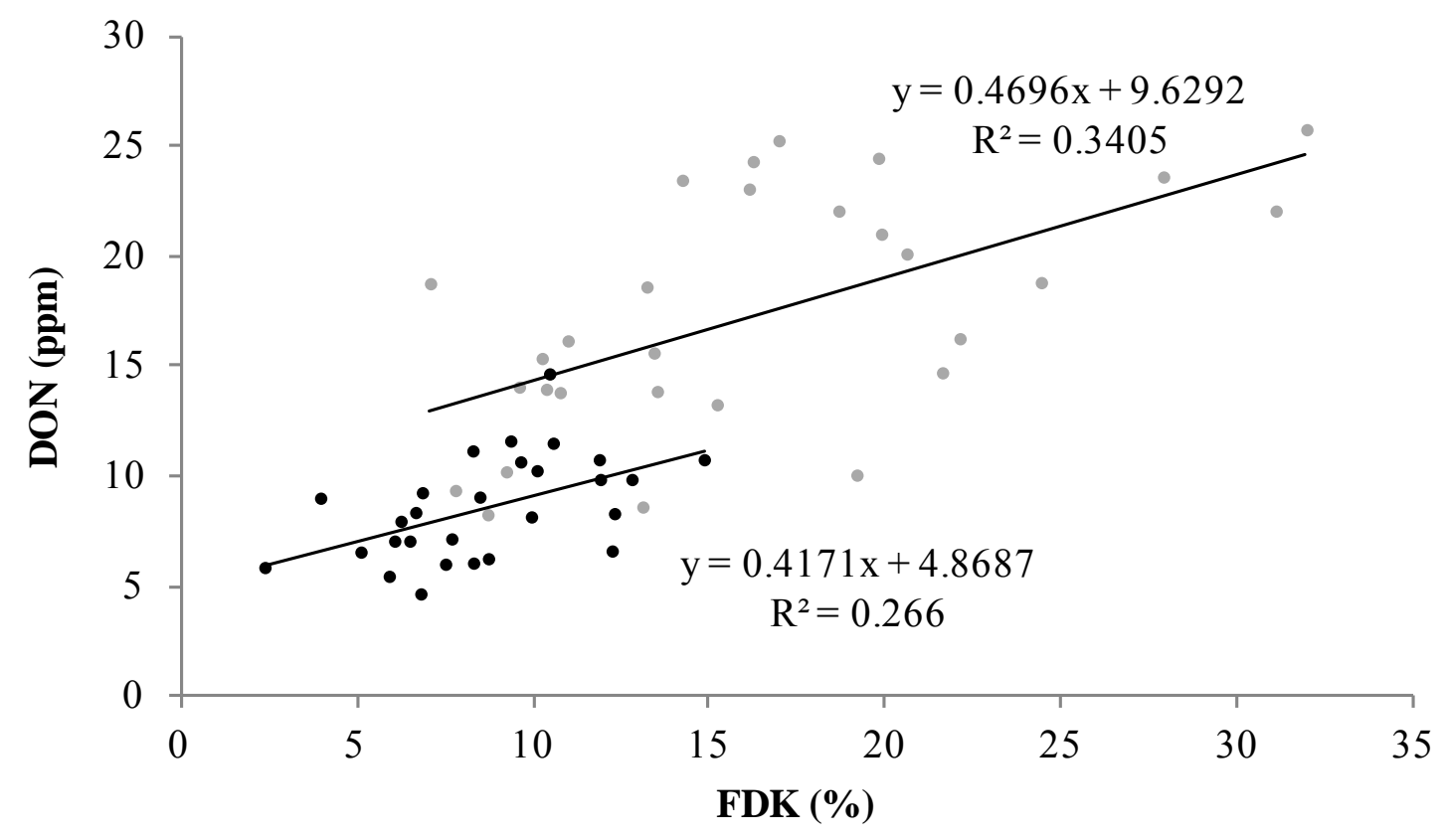


Figure A.3.16: Regression of Fusarium damaged kernels (FDK) on FHB index for all populations in Lexington 2010 and 2011.

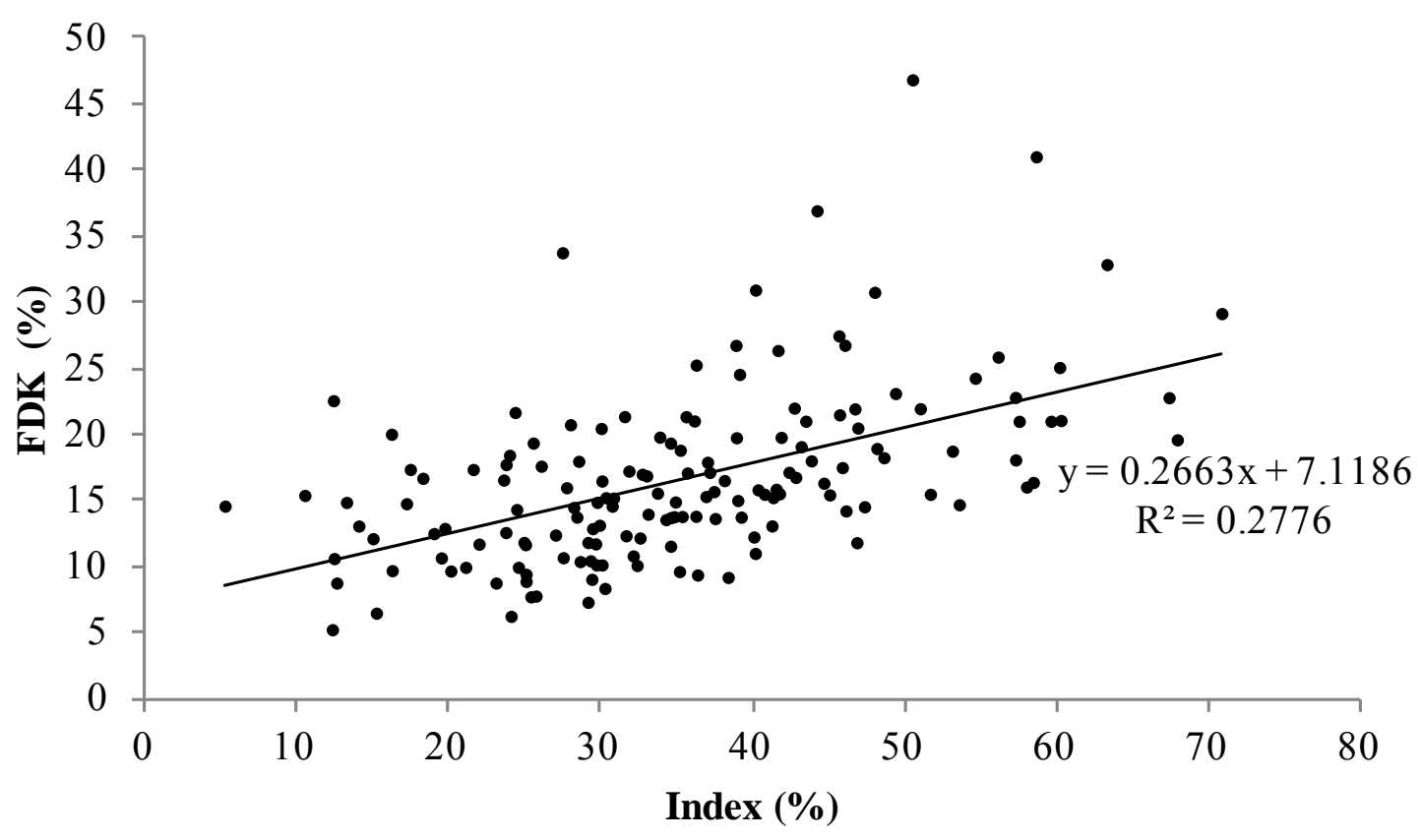


Figure A.3.17: Regression of Fusarium damaged kernels (FDK) on FHB rating for all populations in Lexington 2010 and 2011.

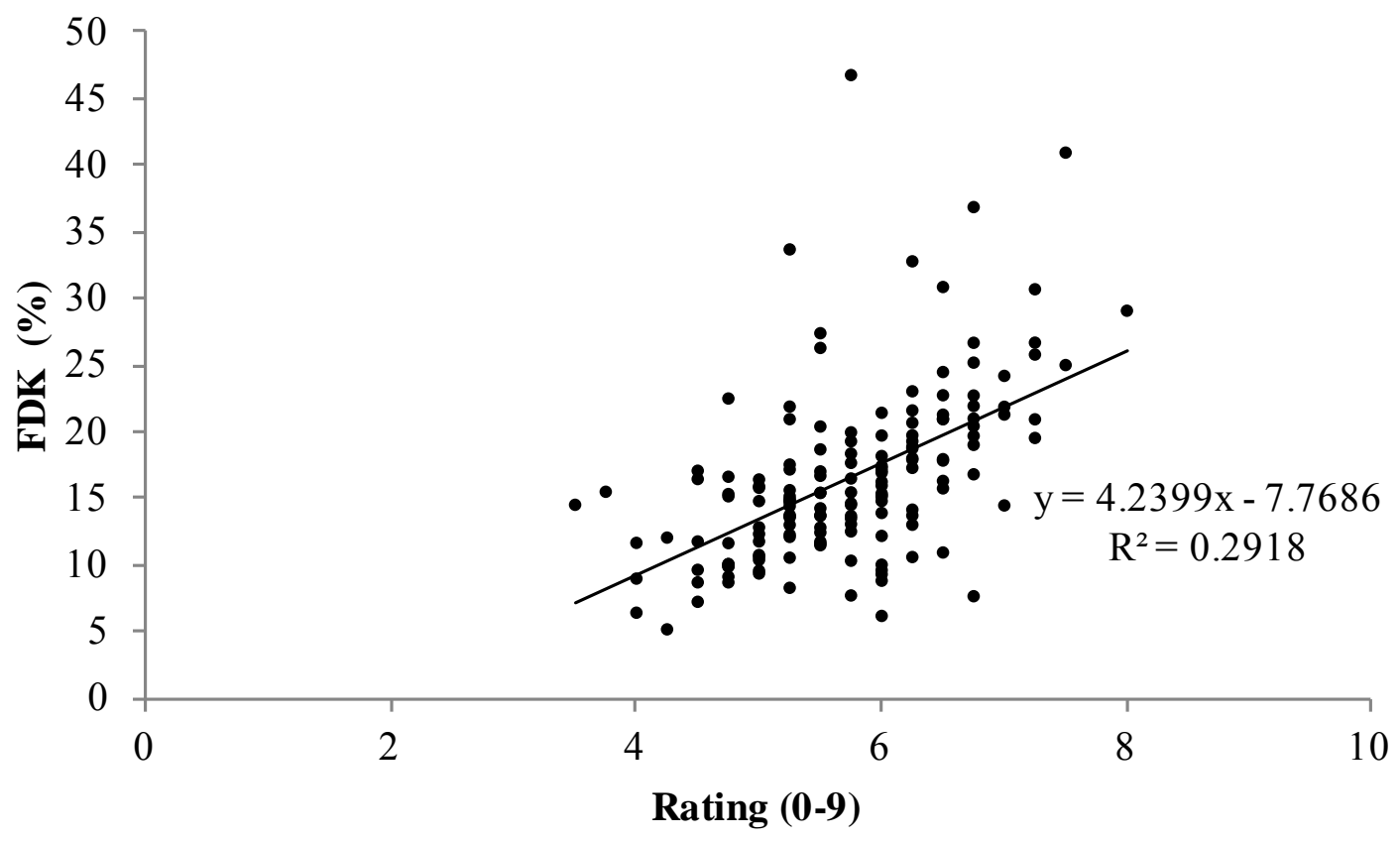


Figure A.3.18: Regression of FHB index on FHB rating for all populations in Lexington 2010 and 2011.

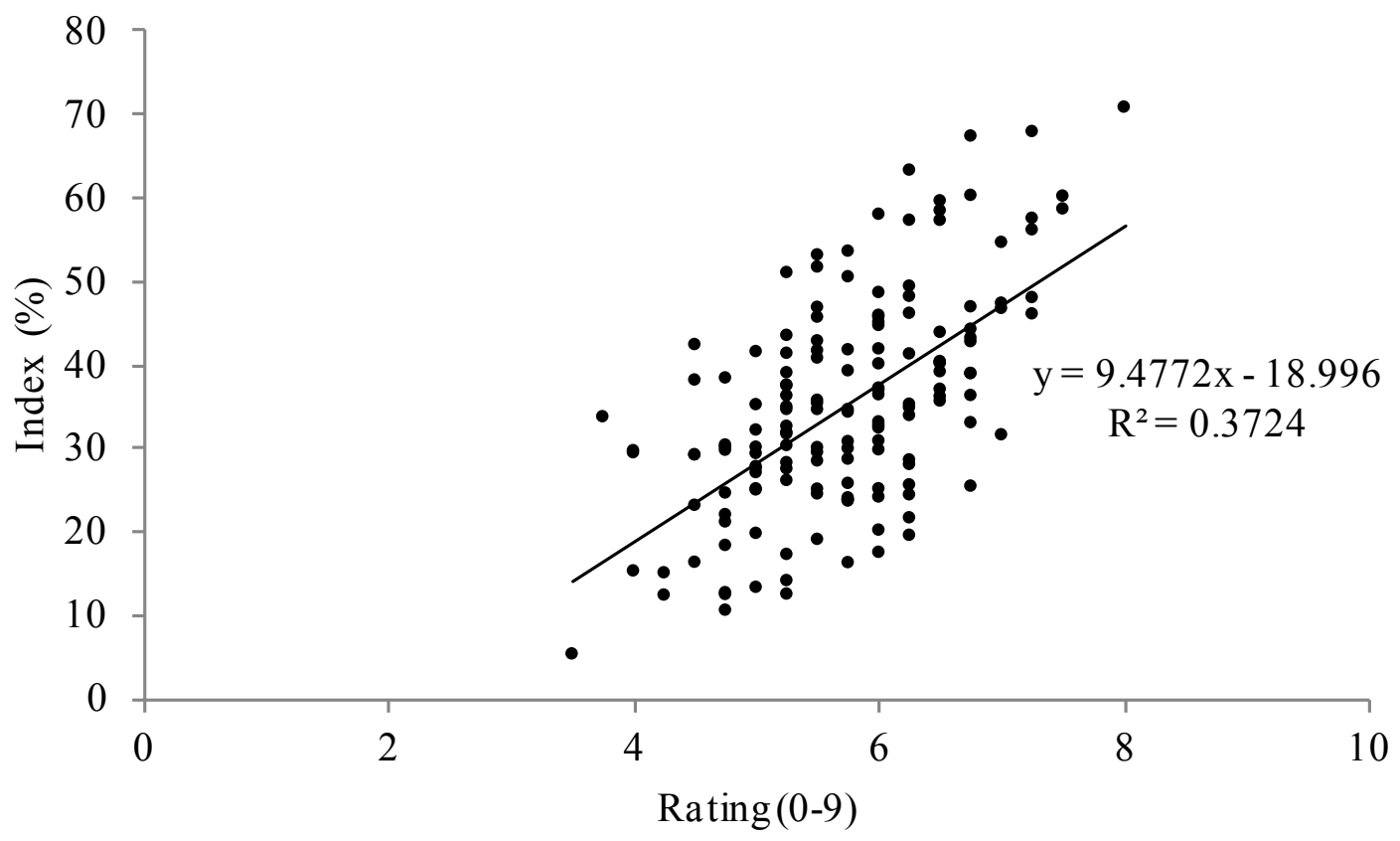


Fig A.3.19: Regression of Fusarium damaged kernels (FDK) on FHB index for all populations in Lexington 2010 and 2011.

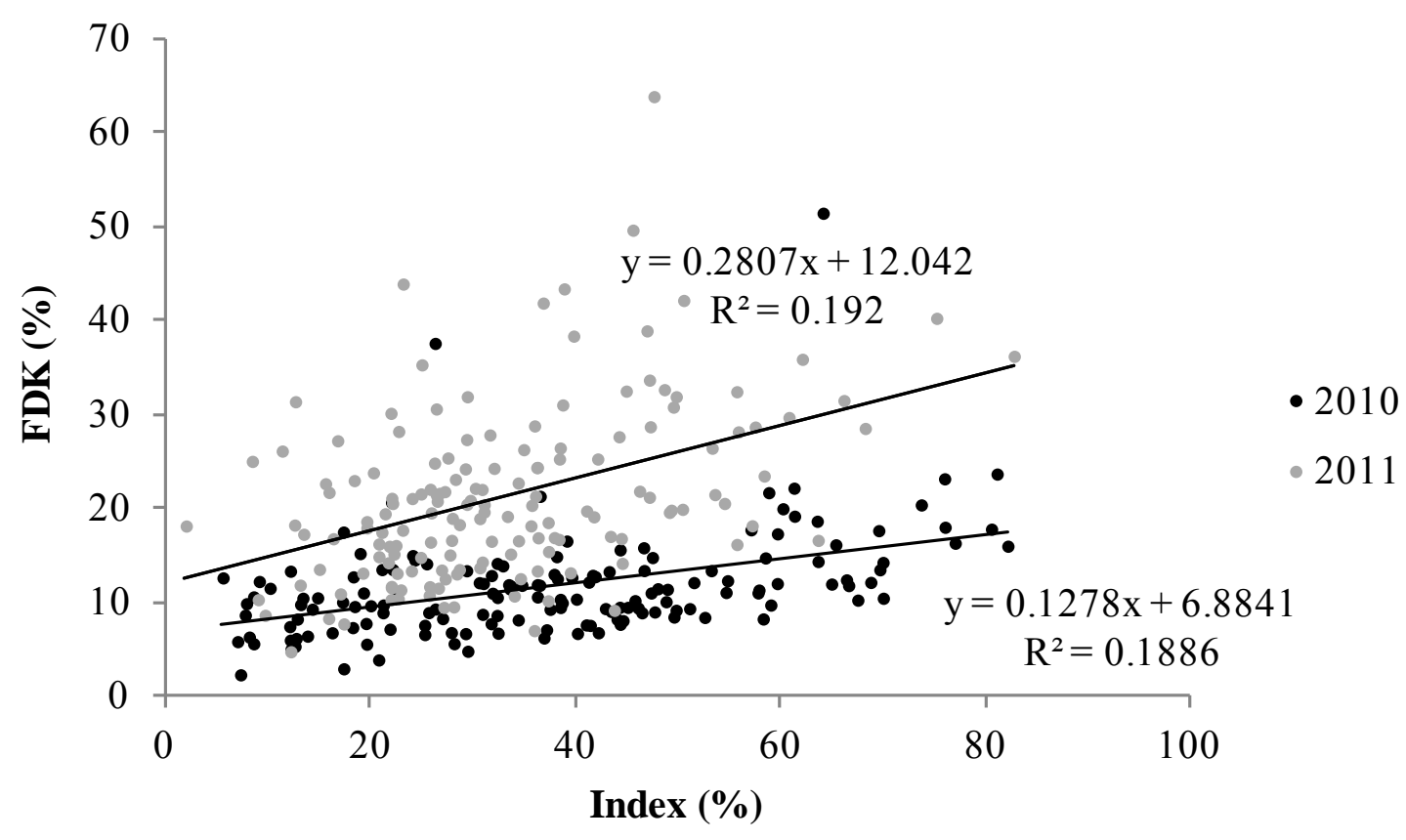


Figure A.3.20: Regression of Fusarium damaged kernels (FDK) on FHB rating for all populations in Lexington 2010 and 2011.

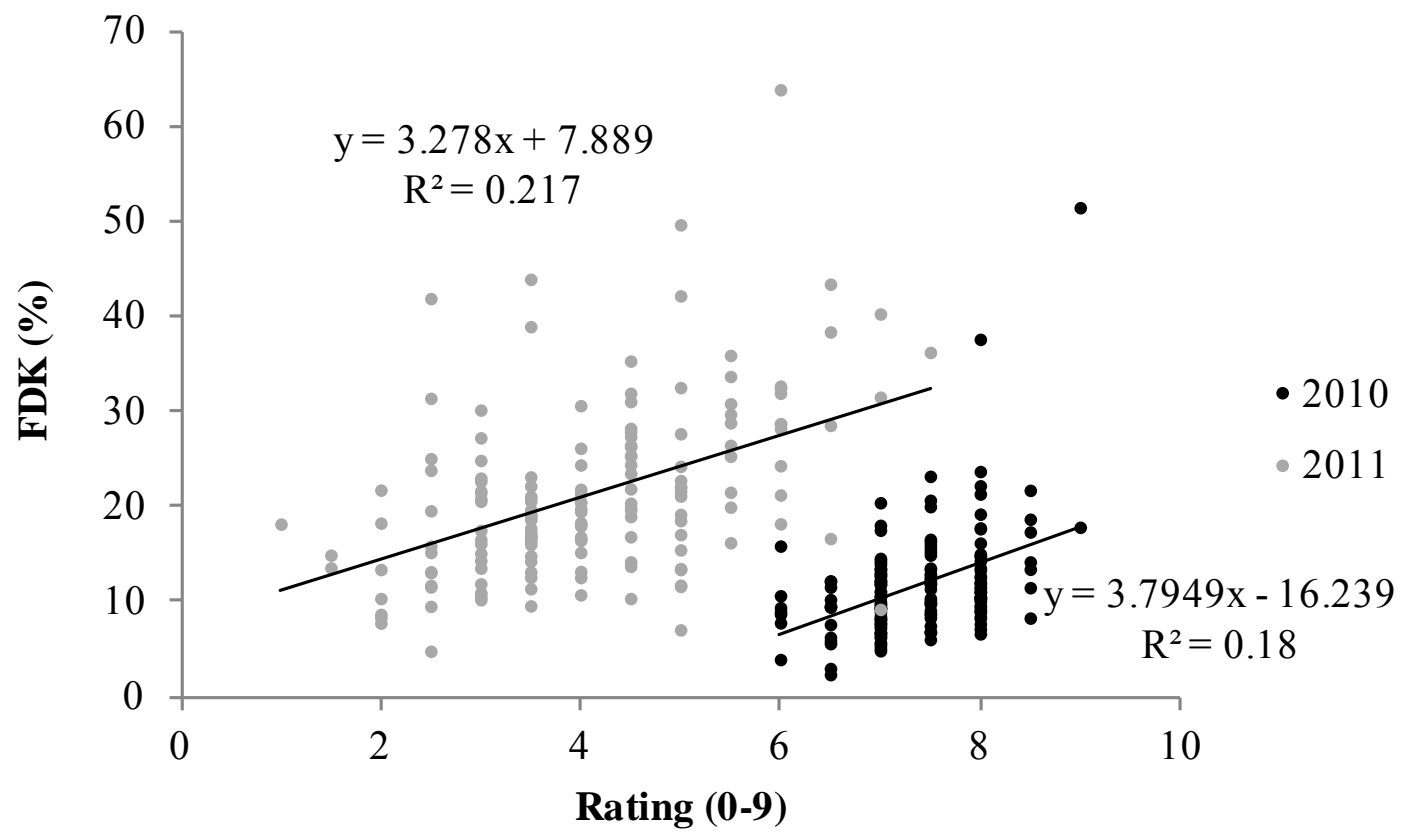


Figure A.3.21: Regression of FHB index on FHB rating for all populations in Lexington 2010 and 2011.

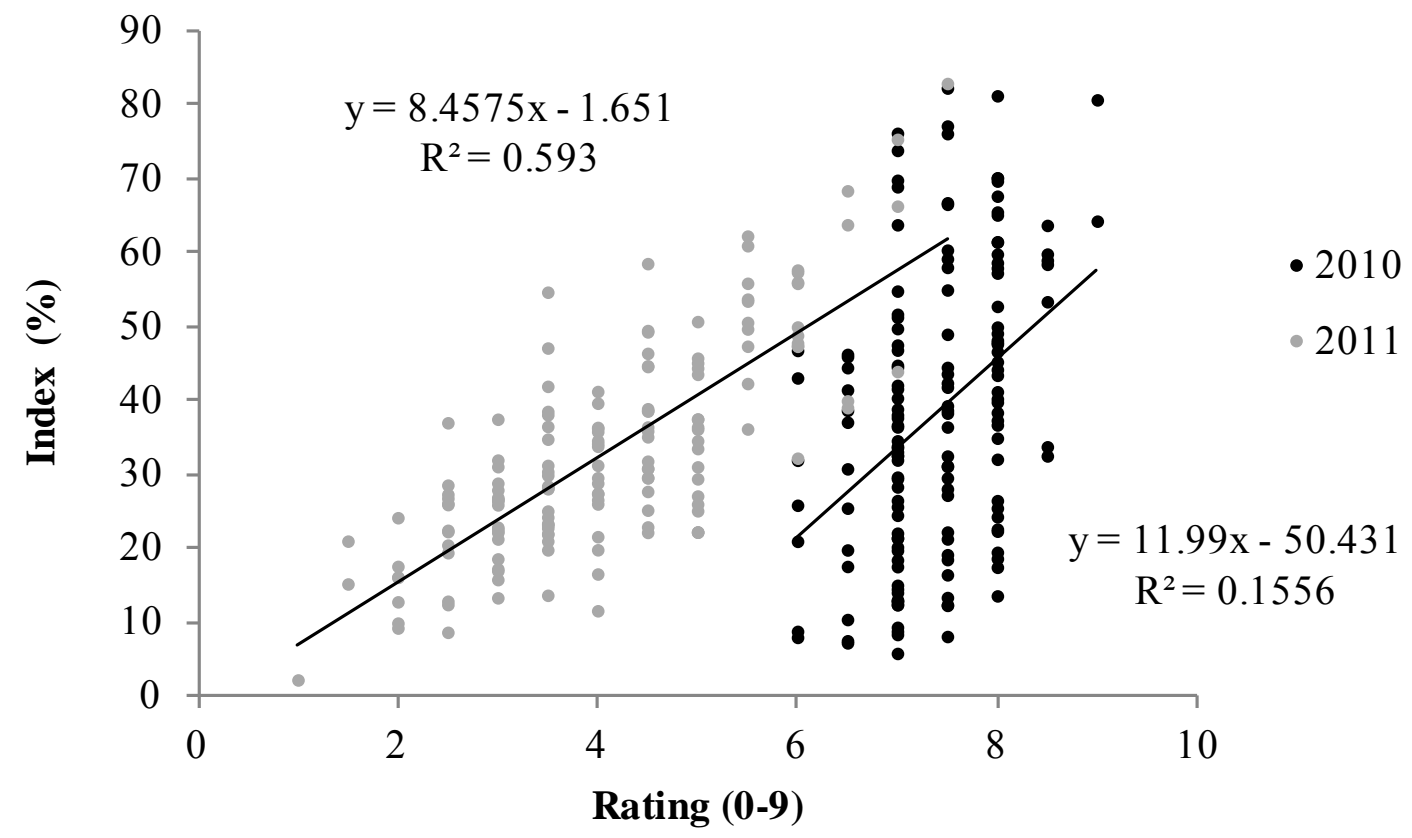


Figure A.4.1: Regression of flour yield on wheat meal (WM) sodium carbonate solvent retention capacity (SRC) in population 1 in Lexington 2010 and Princeton 2011.

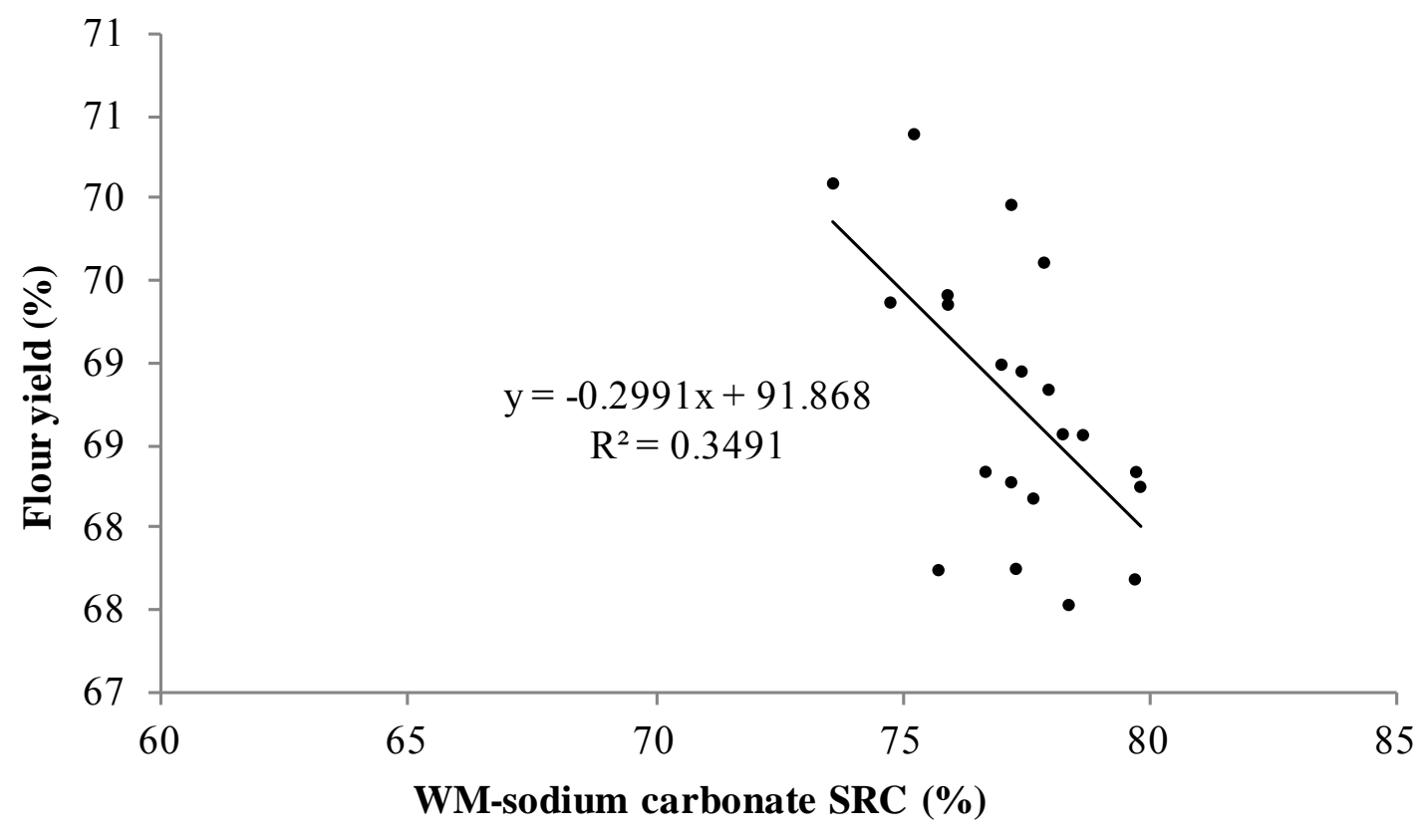


Figure A.4.2: Regression of flour yield on wheat meal (WM) sodium carbonate solvent retention capacity (SRC) in population 2 in Lexington 2010 and Princeton 2011.

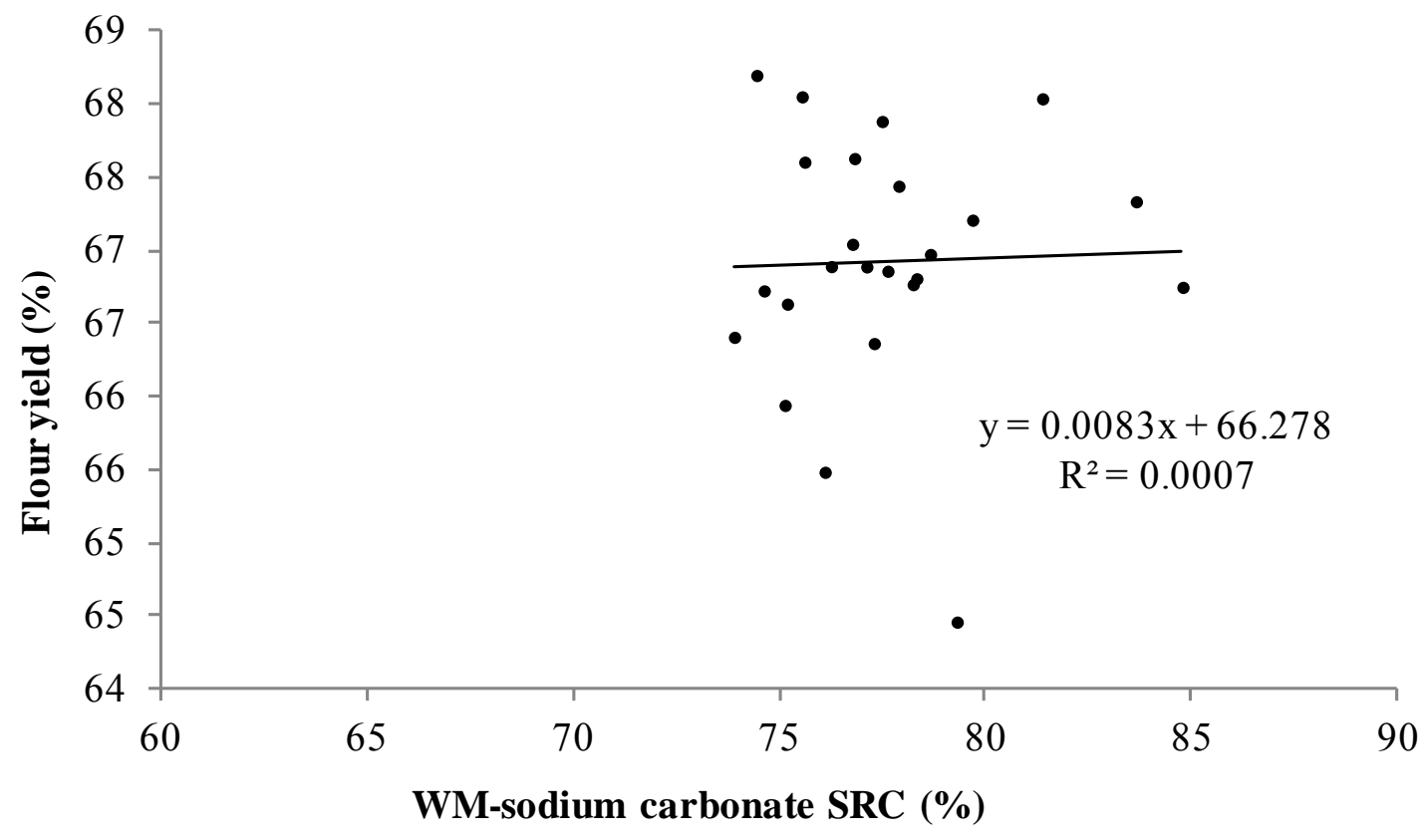


Figure A.4.3: Regression of flour yield on wheat meal (WM) sodium carbonate solvent retention capacity (SRC) in population 3 in Lexington 2010 and Princeton 2011.

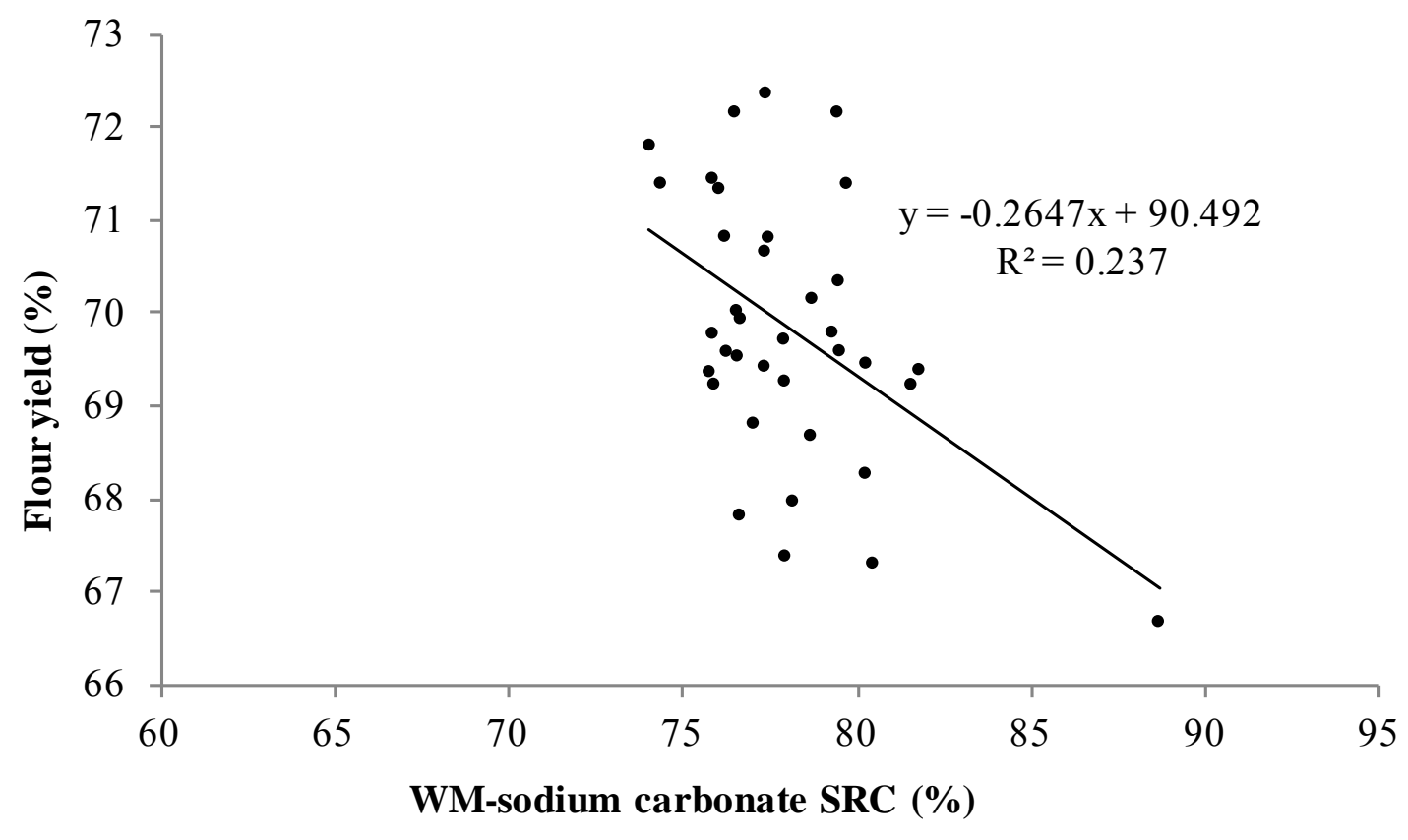


Figure A.4.4: Regression of flour yield on wheat meal (WM) sodium carbonate solvent retention capacity (SRC) in population 4 in Lexington 2010 and Princeton 2011.

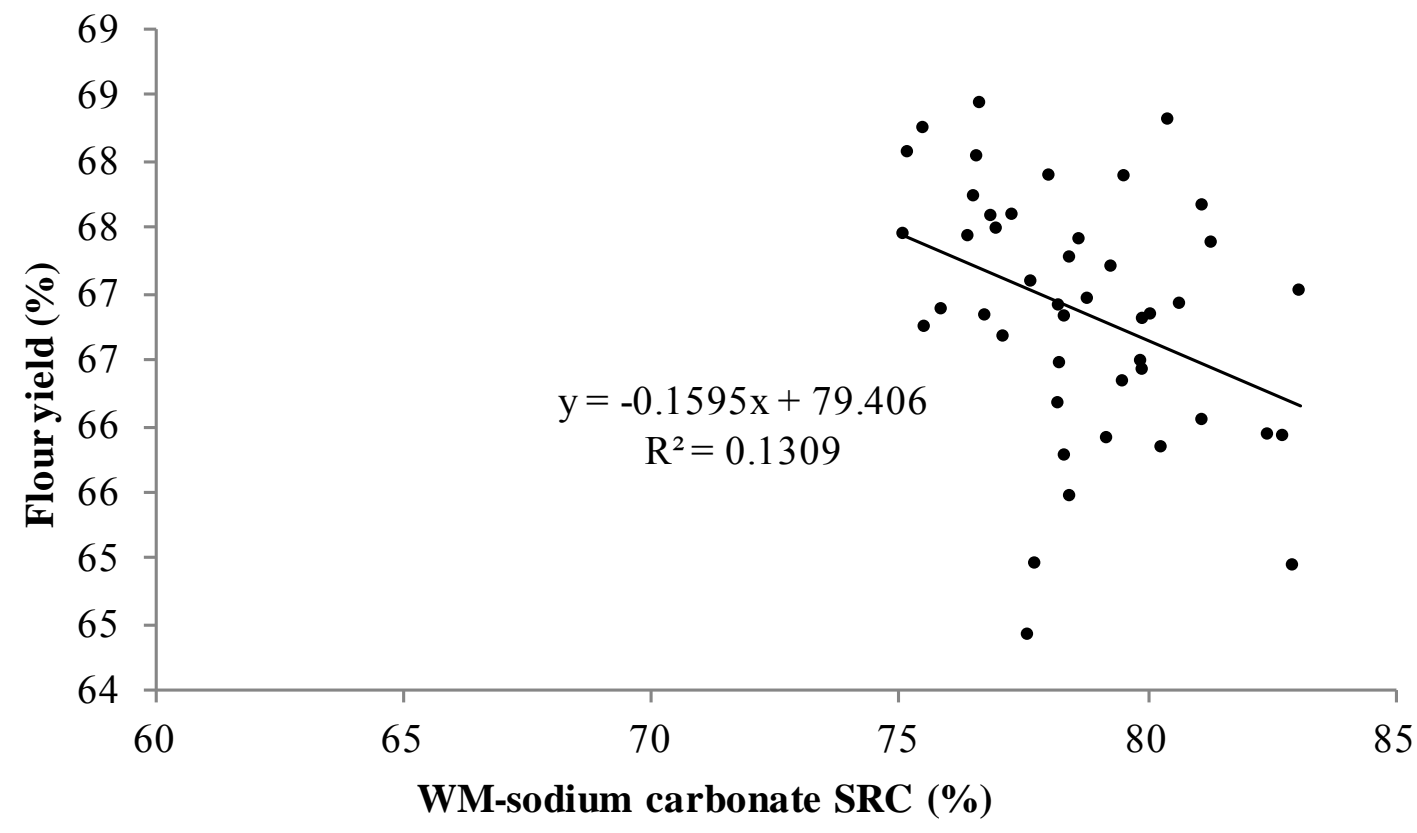


Figure A.4.5: Regression of flour yield on wheat meal (WM) sodium carbonate solvent retention capacity (SRC) in population 5 in Lexington 2010 and Princeton 2011.

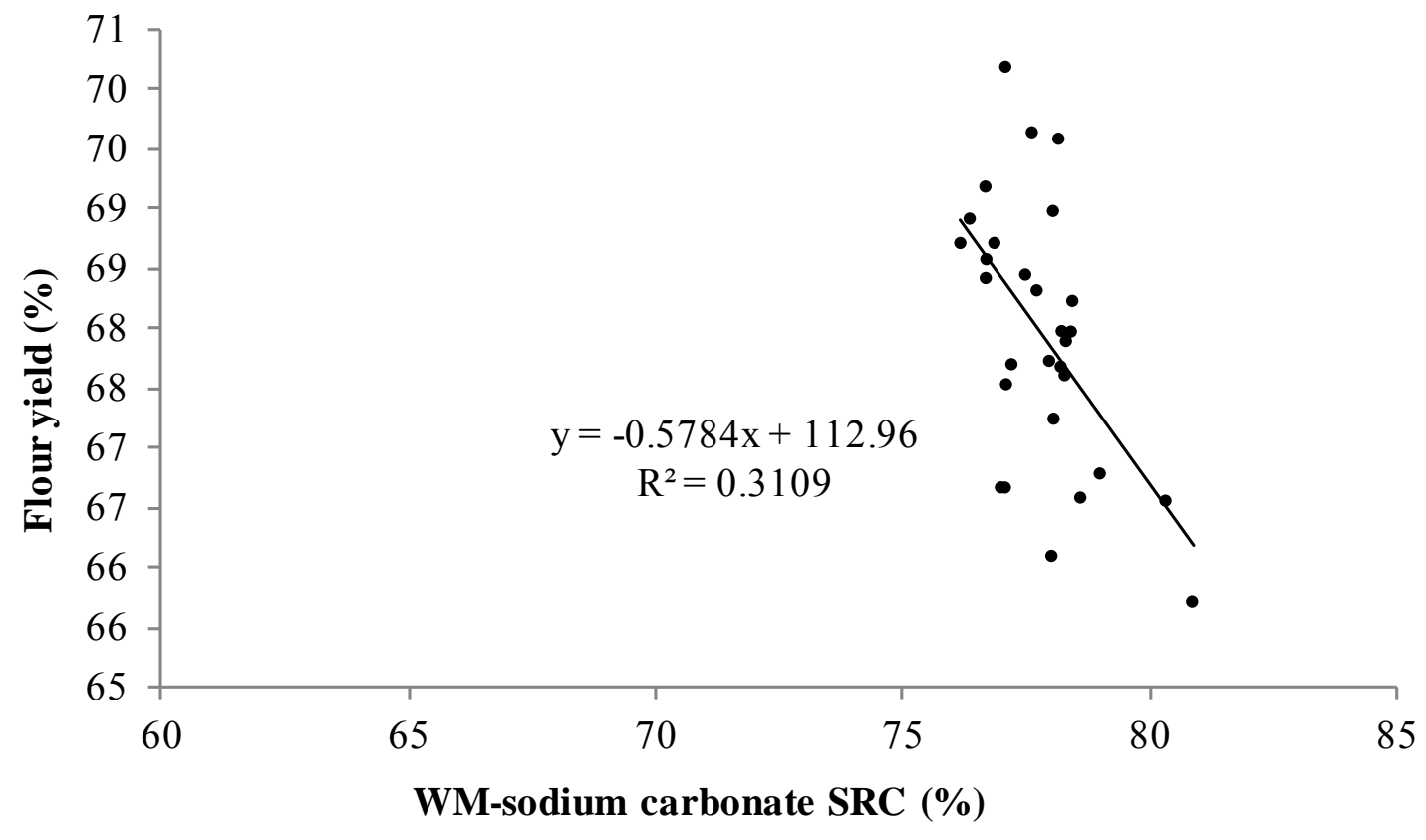


Figure A.4.6: Regression of flour sucrose solvent retention capacity (SRC) on wheat meal (WM) sodium carbonate solvent retention capacity (SRC) in population 1 in Lexington 2010 and Princeton 2011.

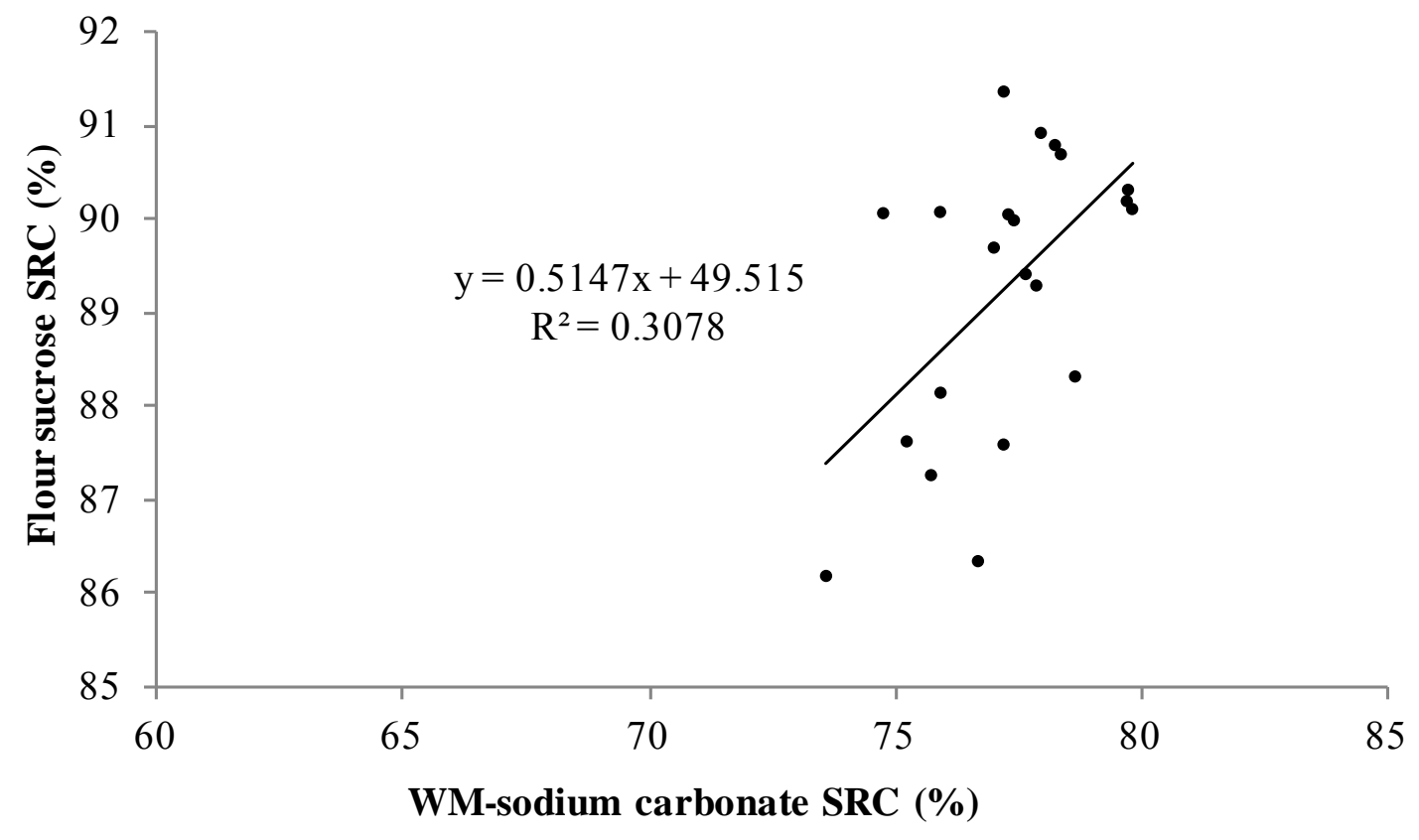


Figure A.4.7: Regression of flour sucrose solvent retention capacity (SRC) on wheat meal (WM) sodium carbonate solvent retention capacity (SRC) in population 2 in Lexington 2010 and Princeton 2011.

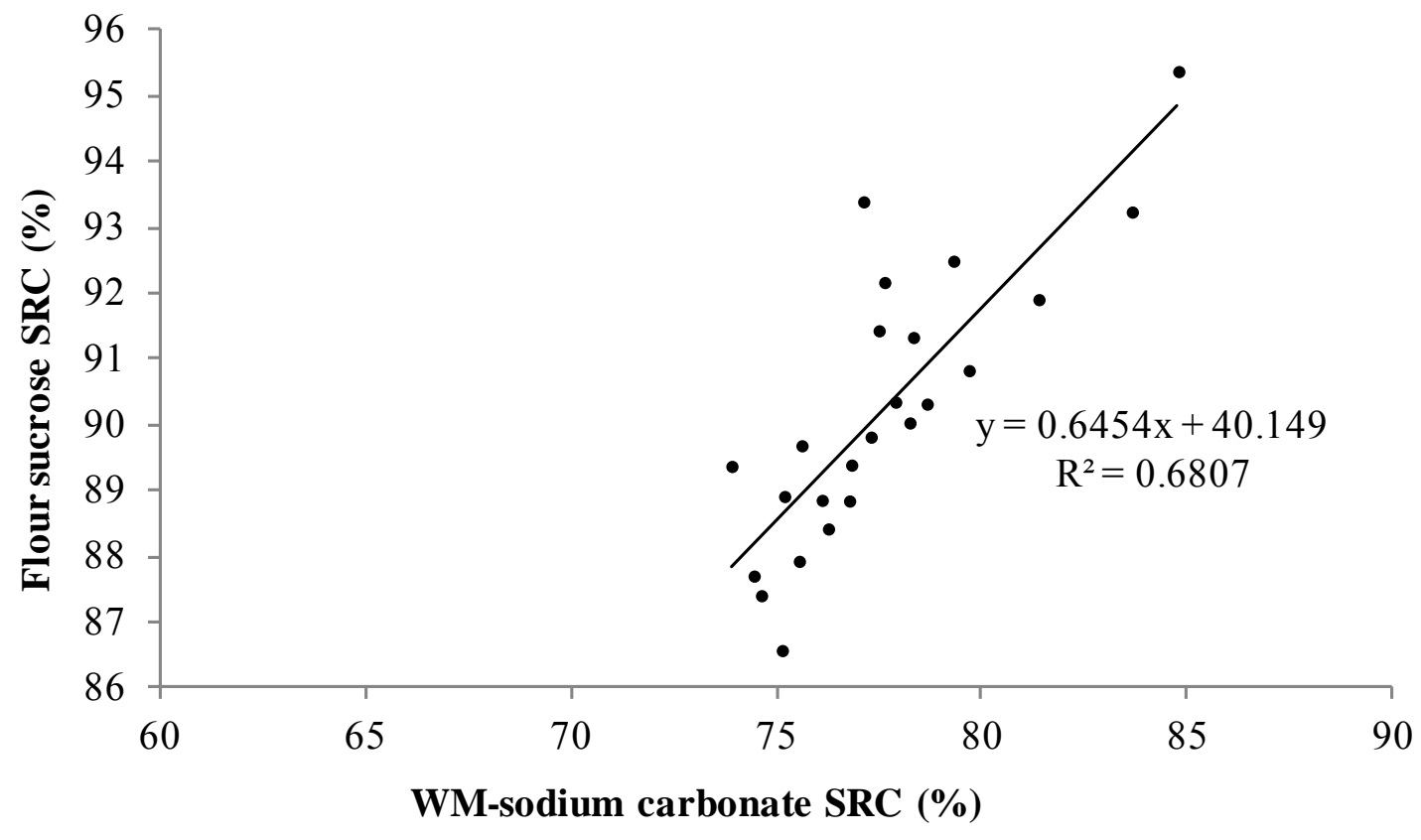


Figure A.4.8: Regression of flour sucrose solvent retention capacity (SRC) on wheat meal (WM) sodium carbonate solvent retention capacity (SRC) in population 3 in Lexington 2010 and Princeton 2011.

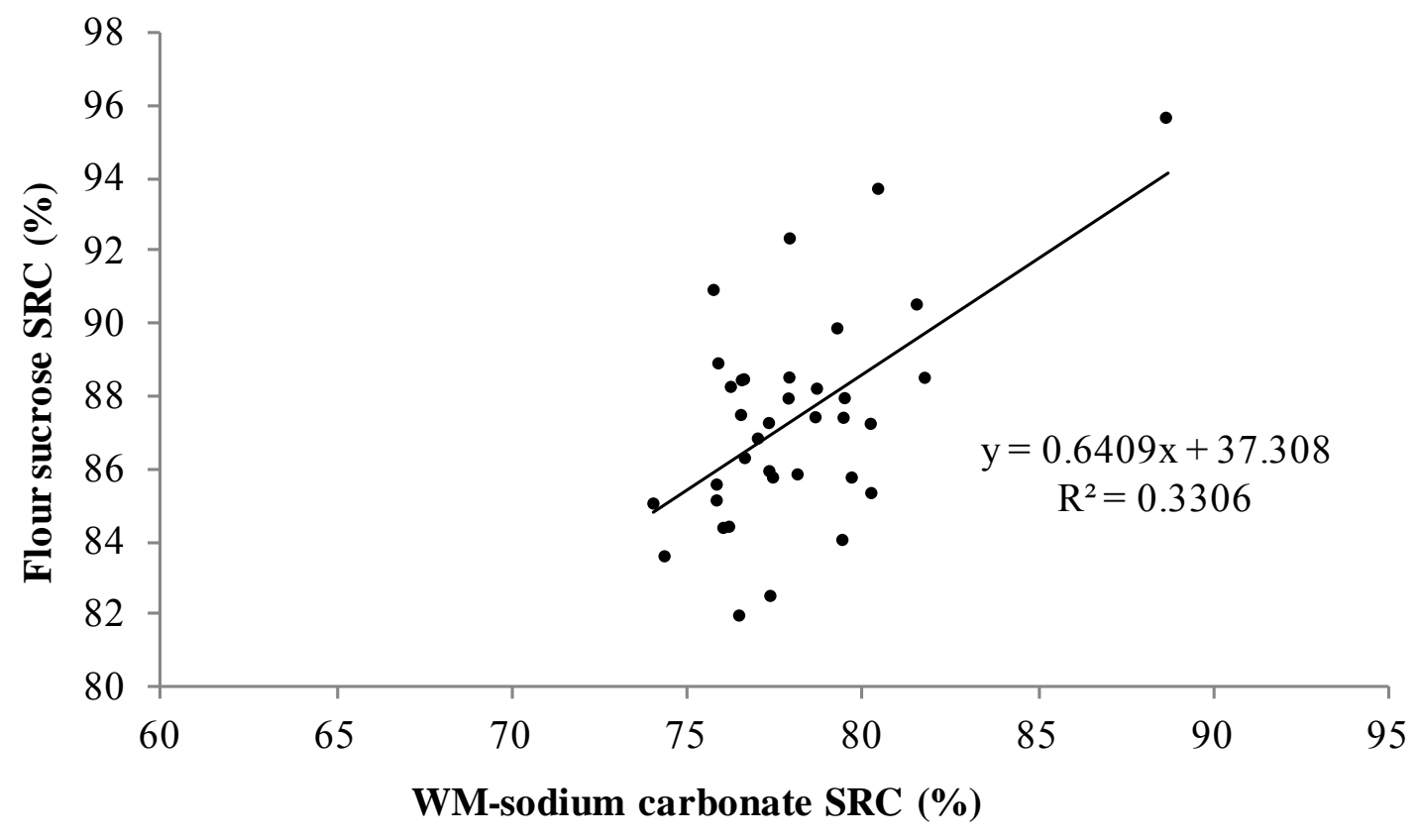


Figure A.4.9: Regression of flour sucrose solvent retention capacity (SRC) on wheat meal (WM) sodium carbonate solvent retention capacity (SRC) in population 4 in Lexington 2010 and Princeton 2011.

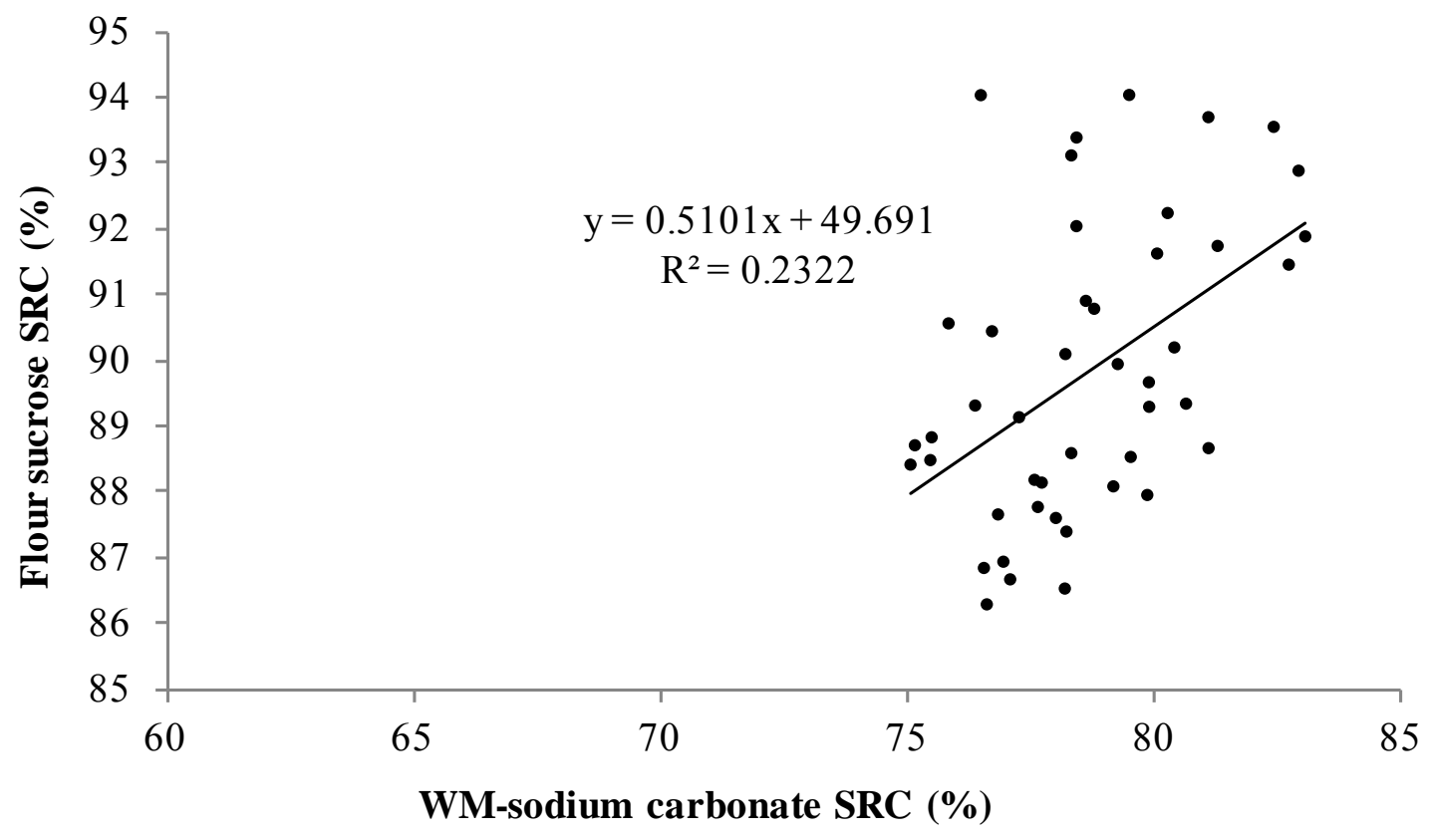


Figure A.4.10: Regression of flour sucrose solvent retention capacity (SRC) on wheat meal (WM) sodium carbonate solvent retention capacity (SRC) in population 5 in Lexington 2010 and Princeton 2011.

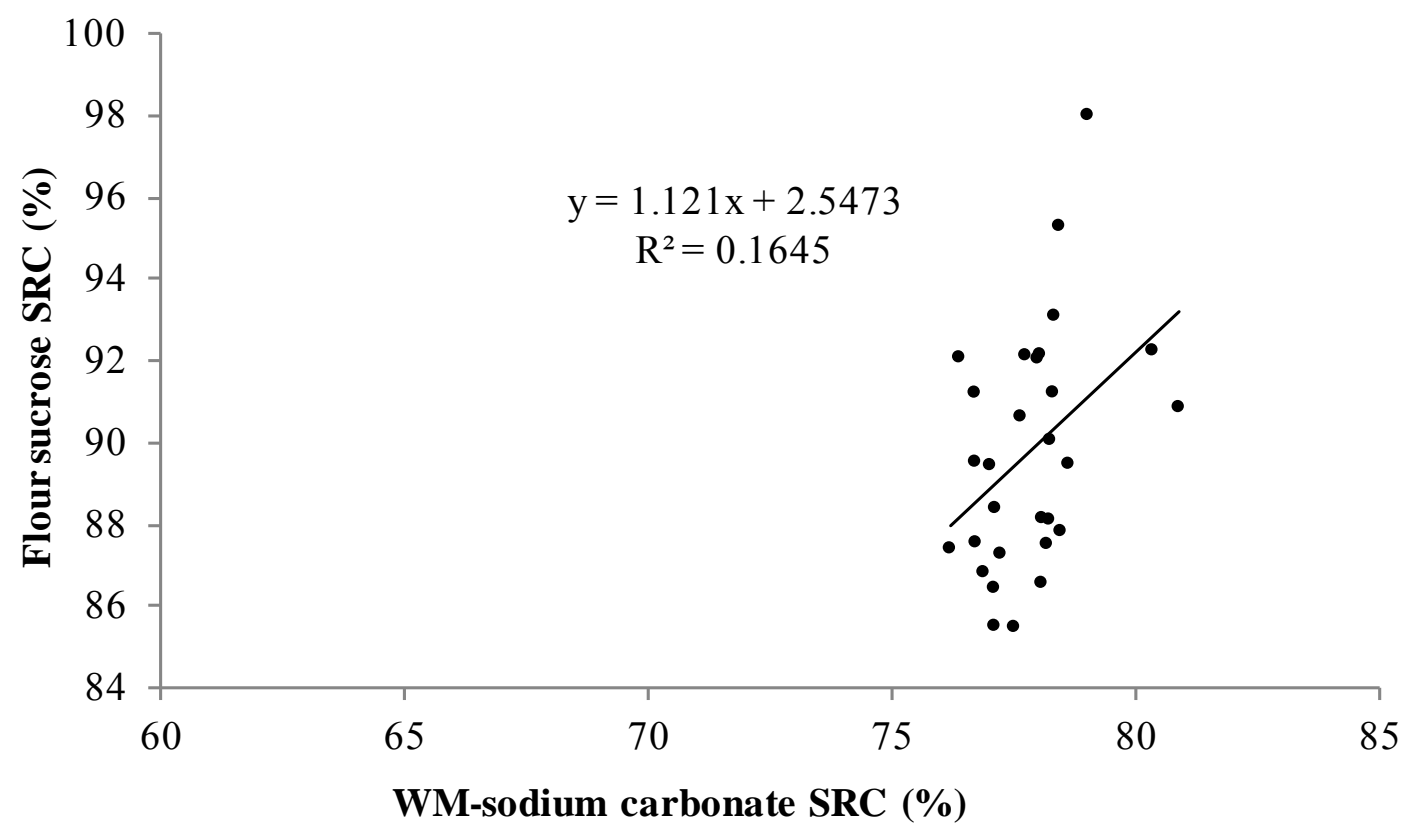


Figure A.4.11: Regression of flour lactic acid solvent retention capacity (SRC) on wheat meal (WM) SDS sedimentation in population 1 in Lexington 2010 and Princeton 2011.

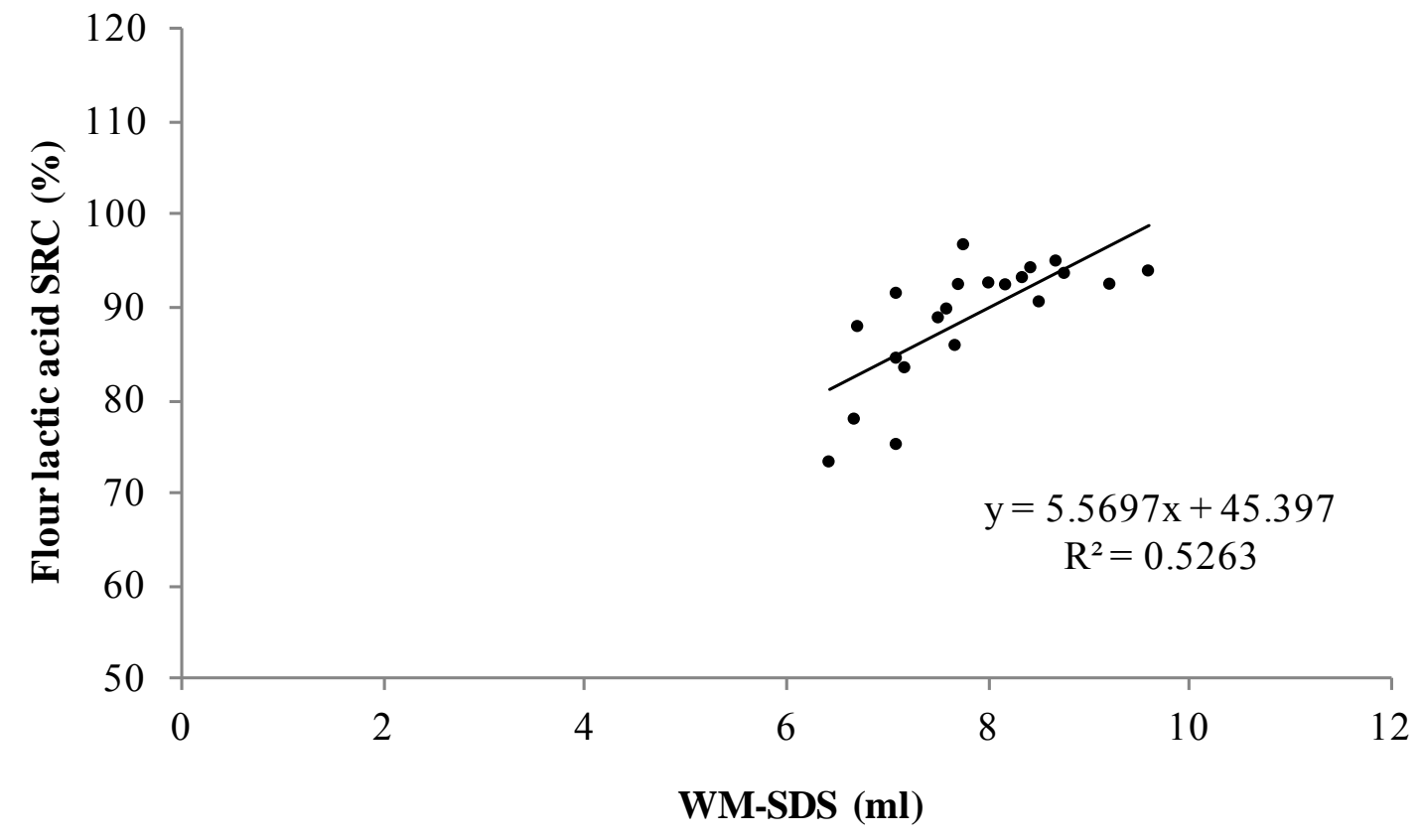


Figure A.4.12: Regression of flour lactic acid solvent retention capacity (SRC) on wheat meal (WM) SDS sedimentation in population 2 in Lexington 2010 and Princeton 2011.

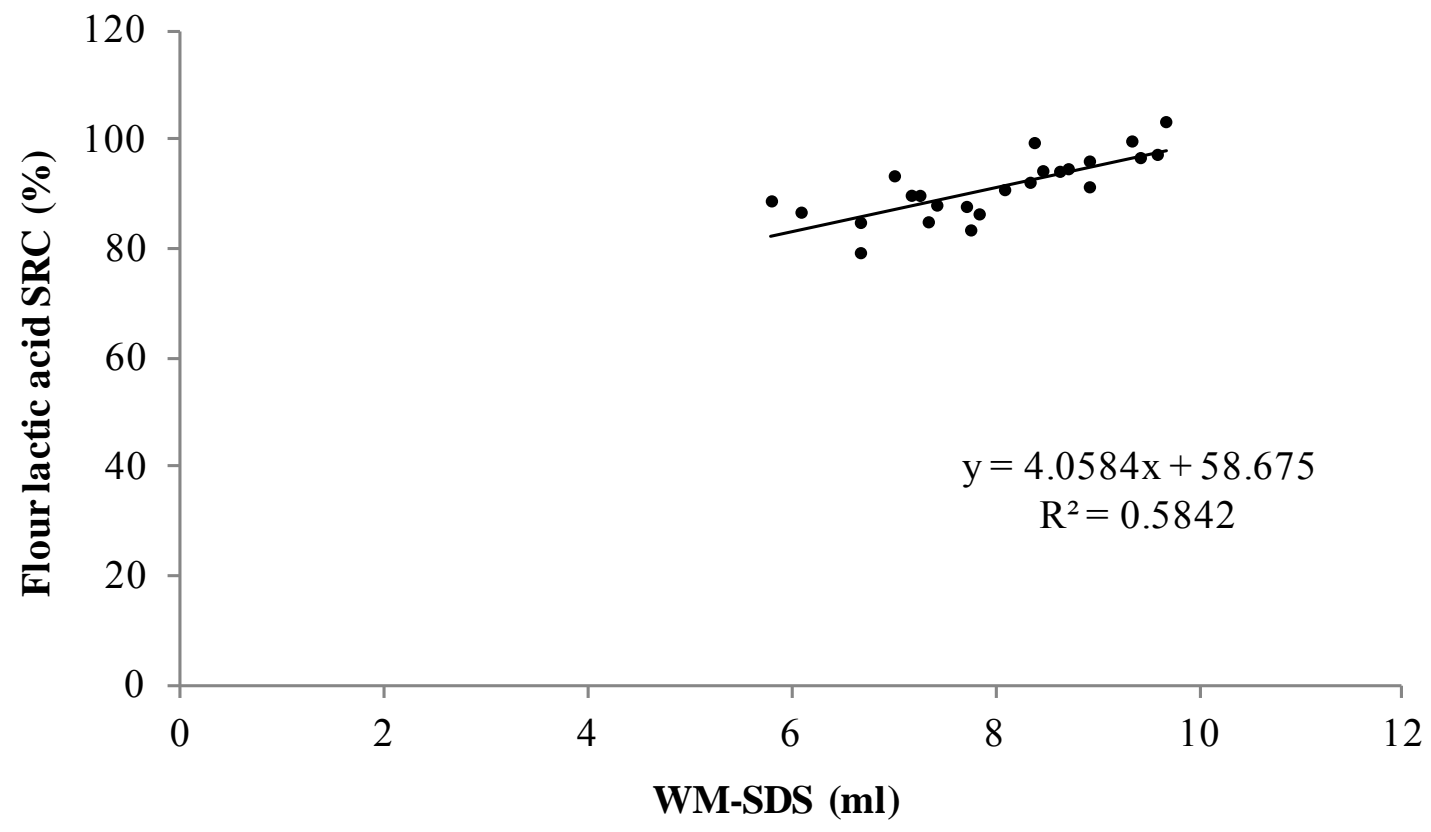


Figure A.4.13: Regression of flour lactic acid solvent retention capacity (SRC) on wheat meal (WM) SDS sedimentation in population 3 in Lexington 2010 and Princeton 2011.

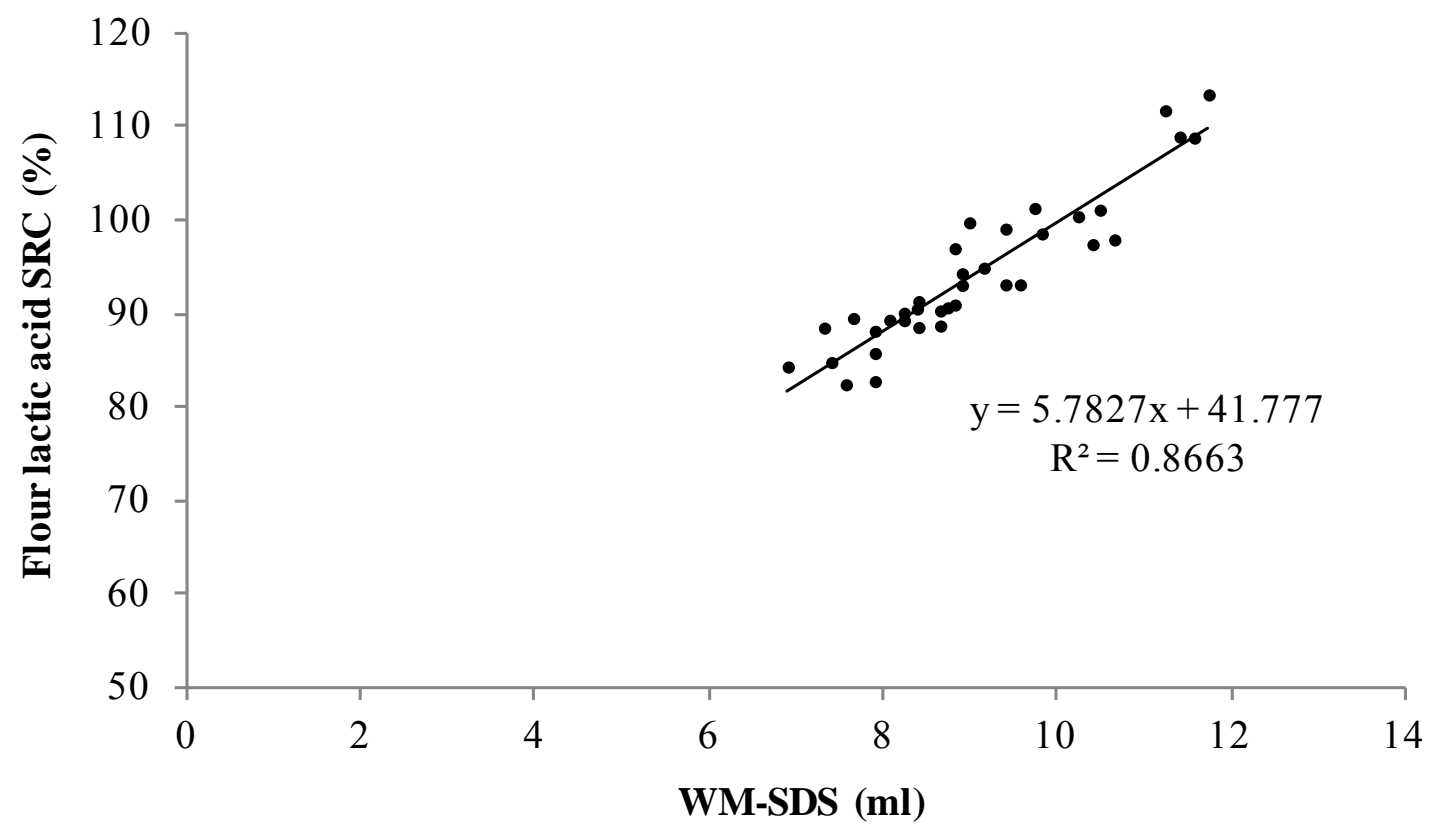


Figure A.4.14: Regression of flour lactic acid solvent retention capacity (SRC) on wheat meal (WM) SDS sedimentation in population 4 in Lexington 2010 and Princeton 2011.

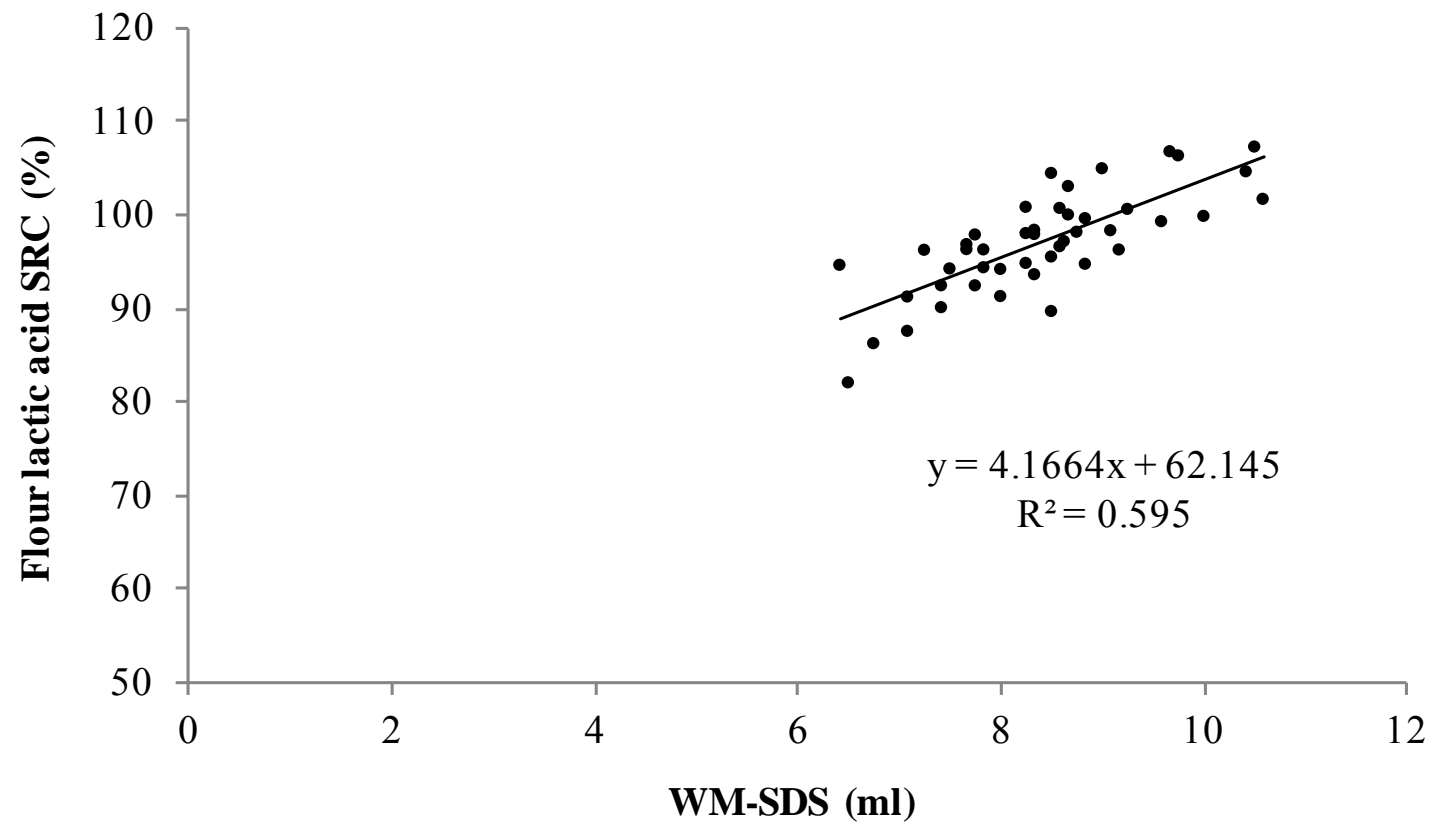


Figure A.4.15: Regression of flour lactic acid solvent retention capacity (SRC) on wheat meal (WM) SDS sedimentation in population 5 in Lexington 2010 and Princeton 2011.

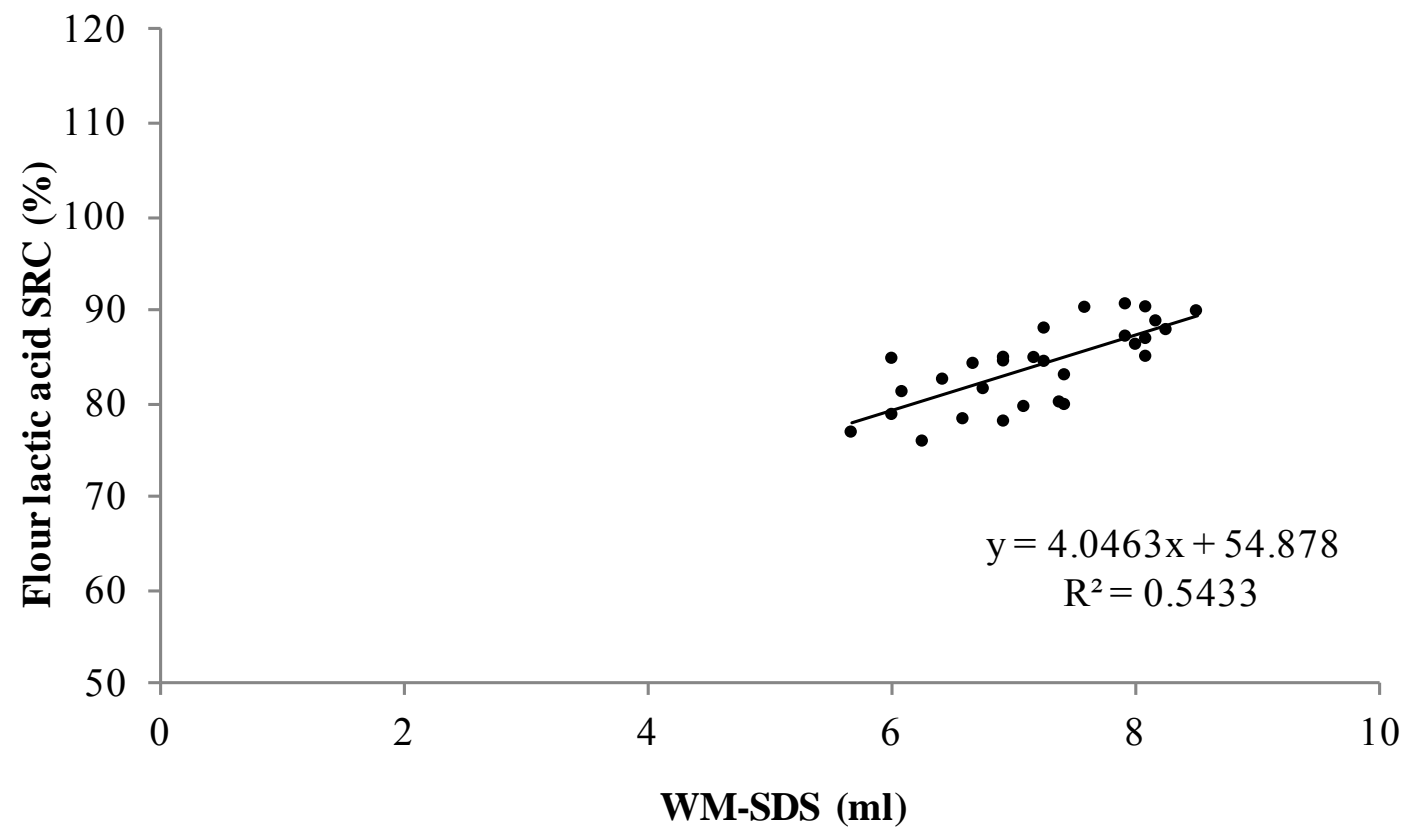


Figure A.4.16: Regression of softness equivalent on NIR softness equivalent in population 1 in Lexington 2010 and Princeton 2011.

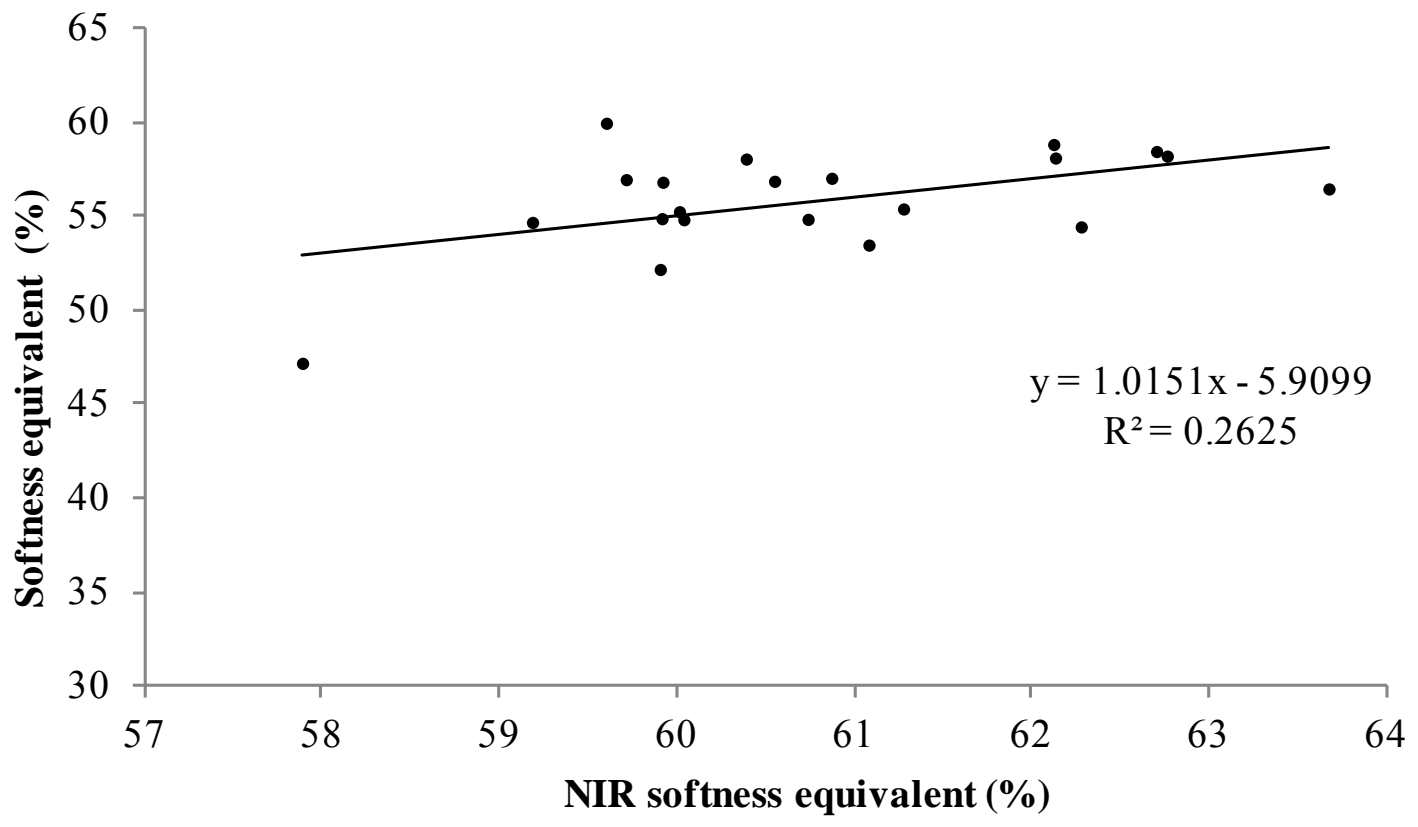


Figure A.4.17: Regression of softness equivalent on NIR softness equivalent in population 2 in Lexington 2010 and Princeton 2011.

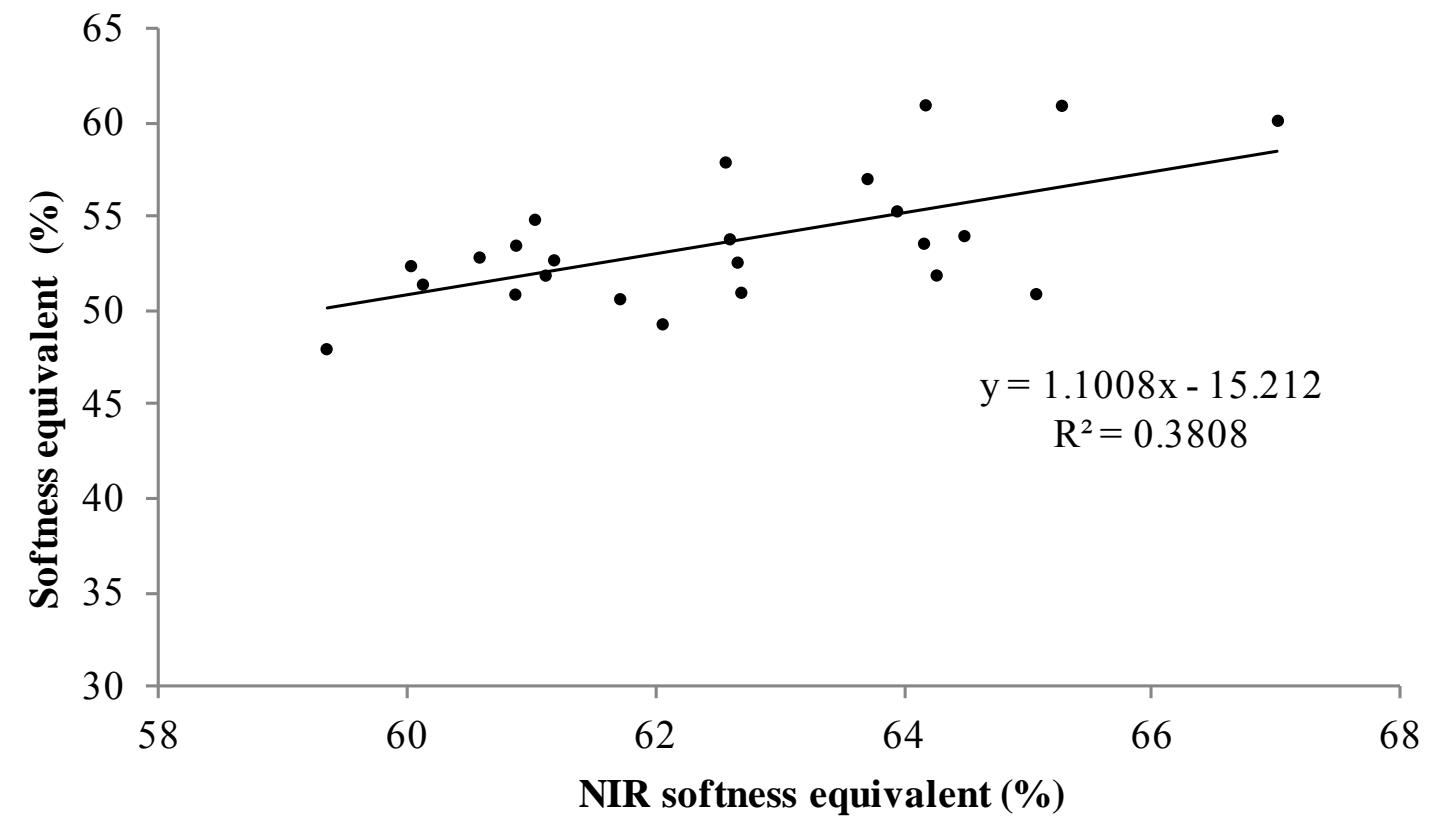


Figure A.4.18: Regression of softness equivalent on NIR softness equivalent in population 3 in Lexington 2010 and Princeton 2011.

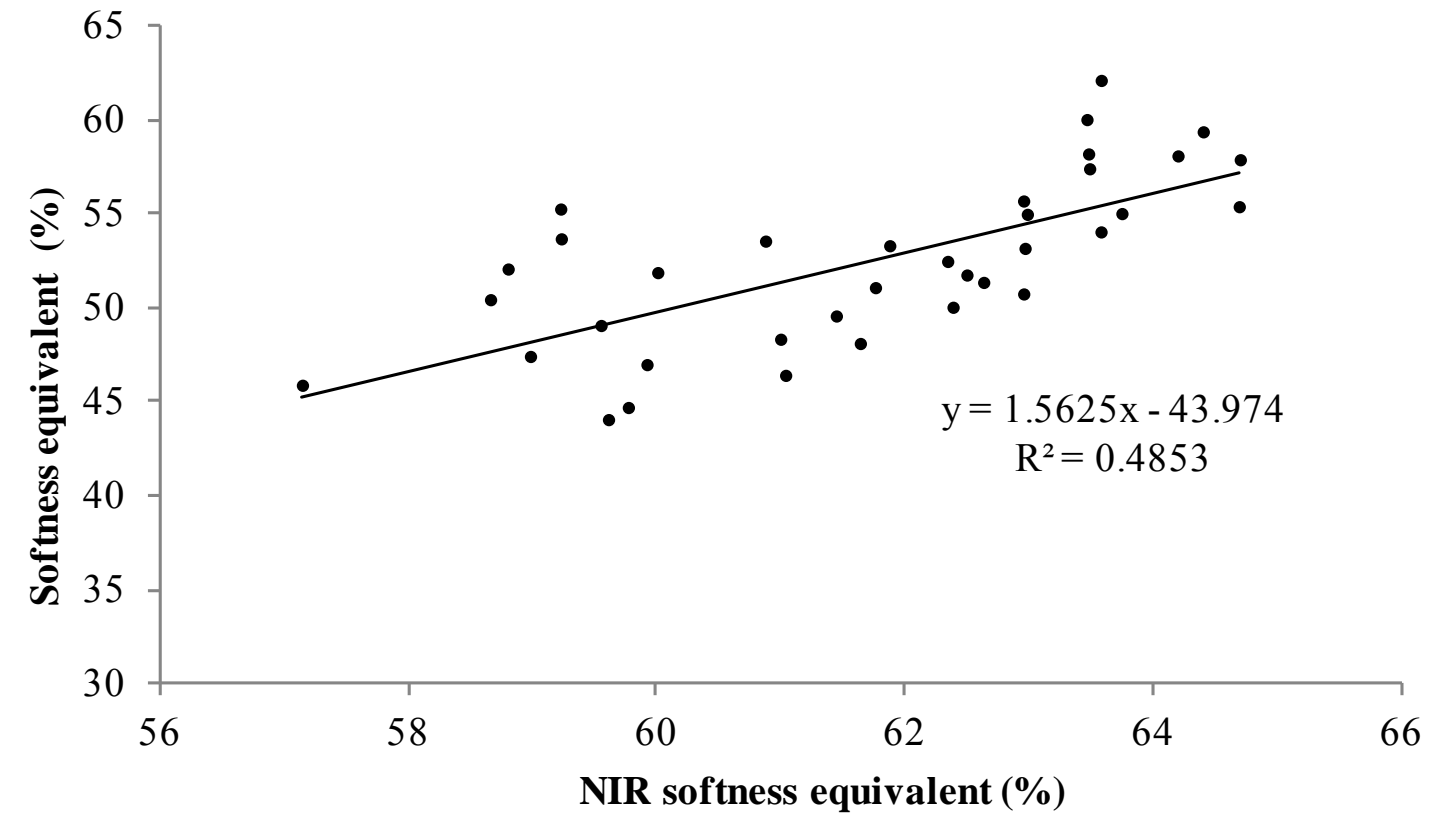


Figure A.4.19: Regression of softness equivalent on NIR softness equivalent in population 4 in Lexington 2010 and Princeton 2011.

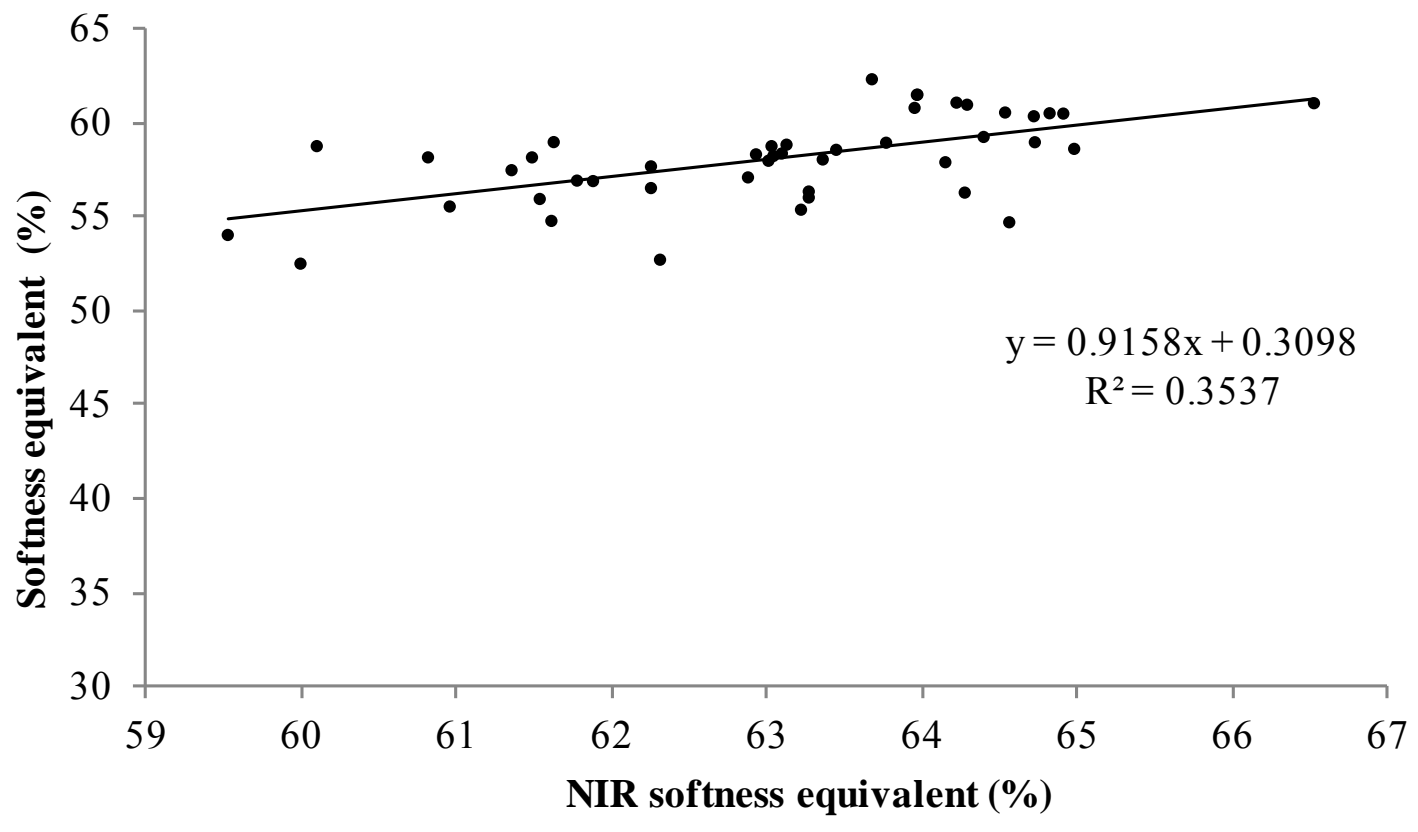


Figure A.4.20: Regression of softness equivalent on NIR softness equivalent in population 5 in Lexington 2010 and Princeton 2011.

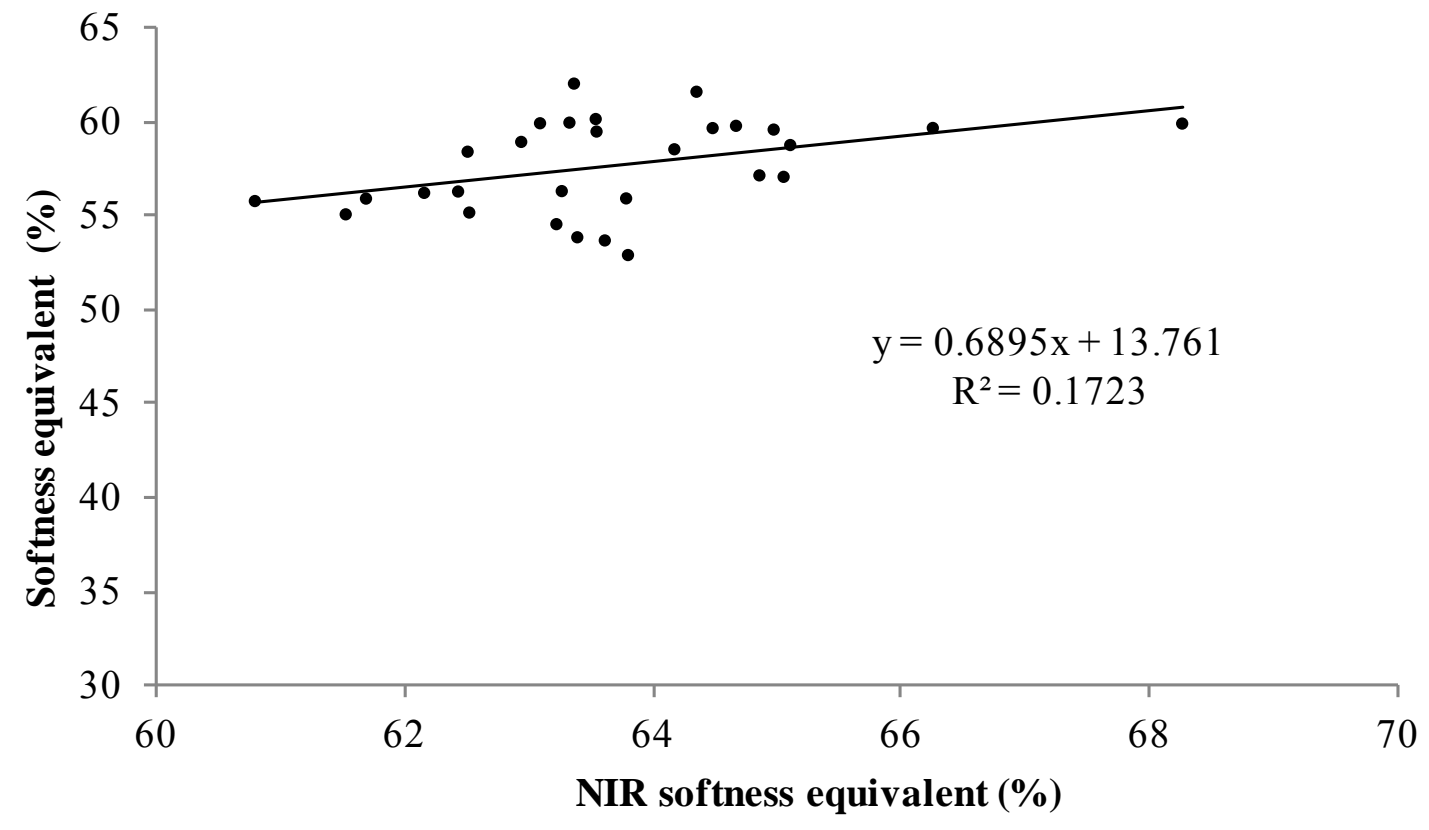


Figure A.4.21: Regression of flour water solvent retention capacity (SRC) on NIR water SRC in population 1 in Lexington 2010 and Princeton 2011.

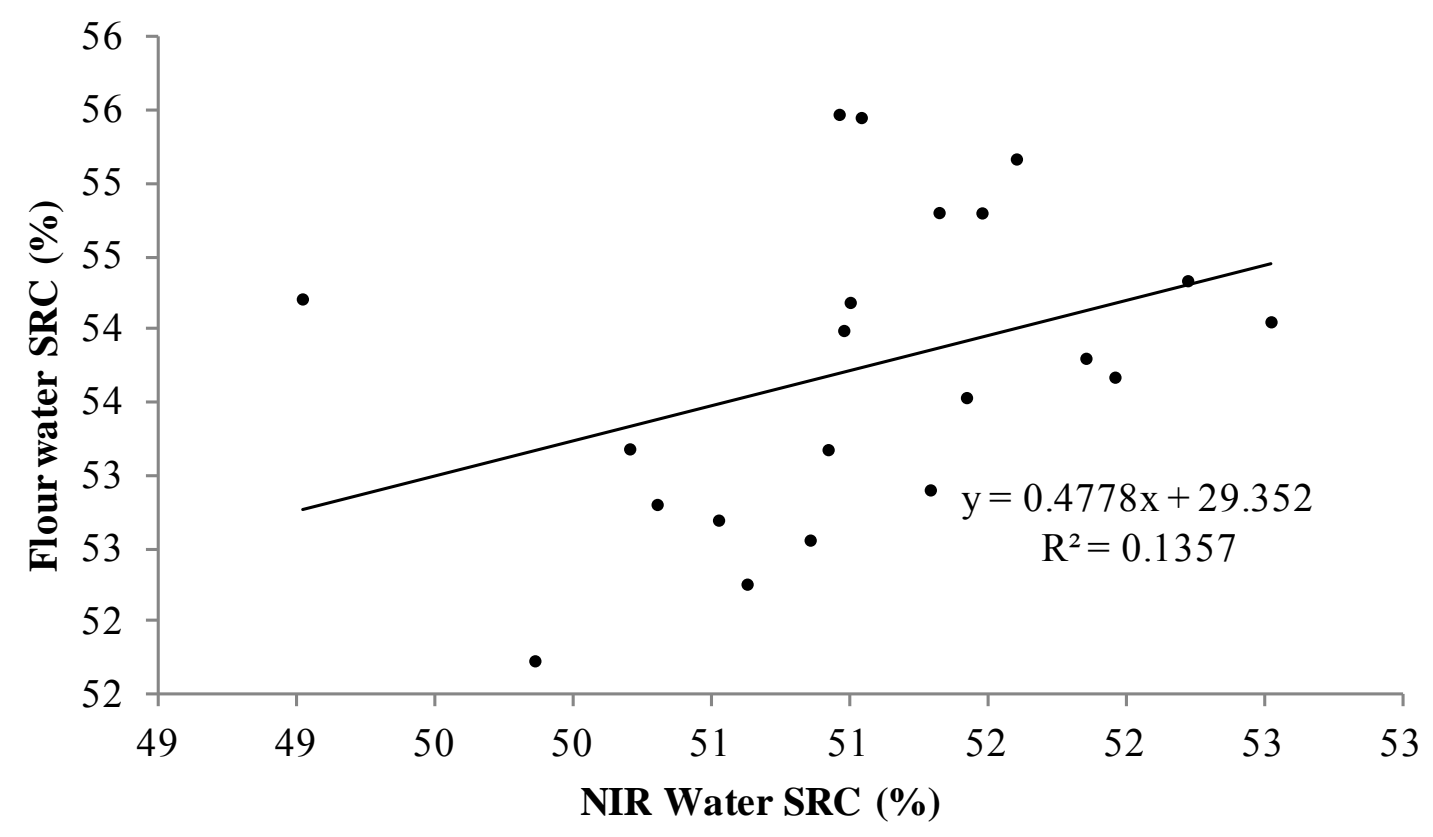


Figure A.4.22: Regression of flour water solvent retention capacity (SRC) on NIR water SRC in population 2 in Lexington 2010 and Princeton 2011.

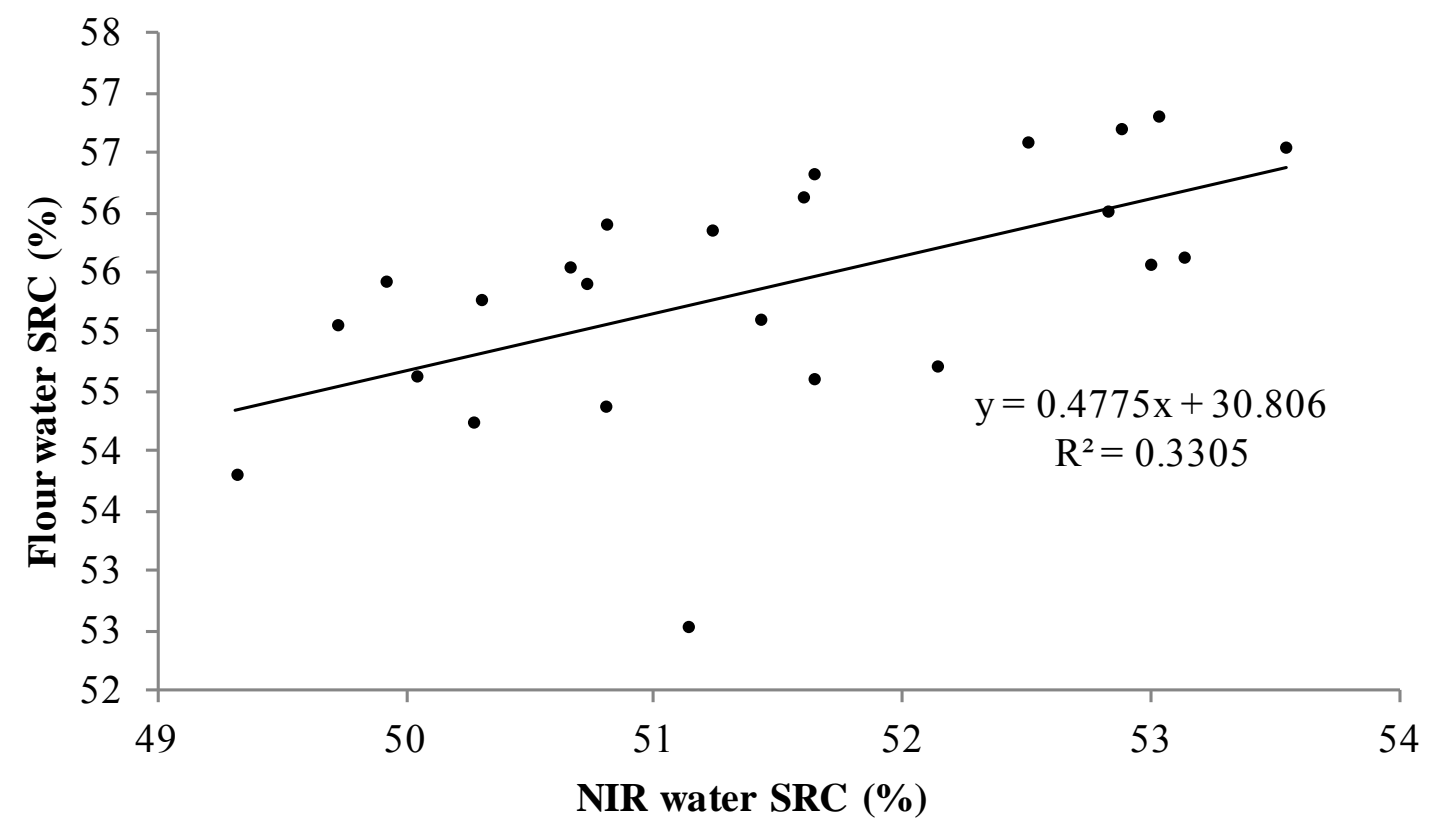


Figure A.4.23: Regression of flour water solvent retention capacity (SRC) on NIR water SRC in population 3 in Lexington 2010 and Princeton 2011.

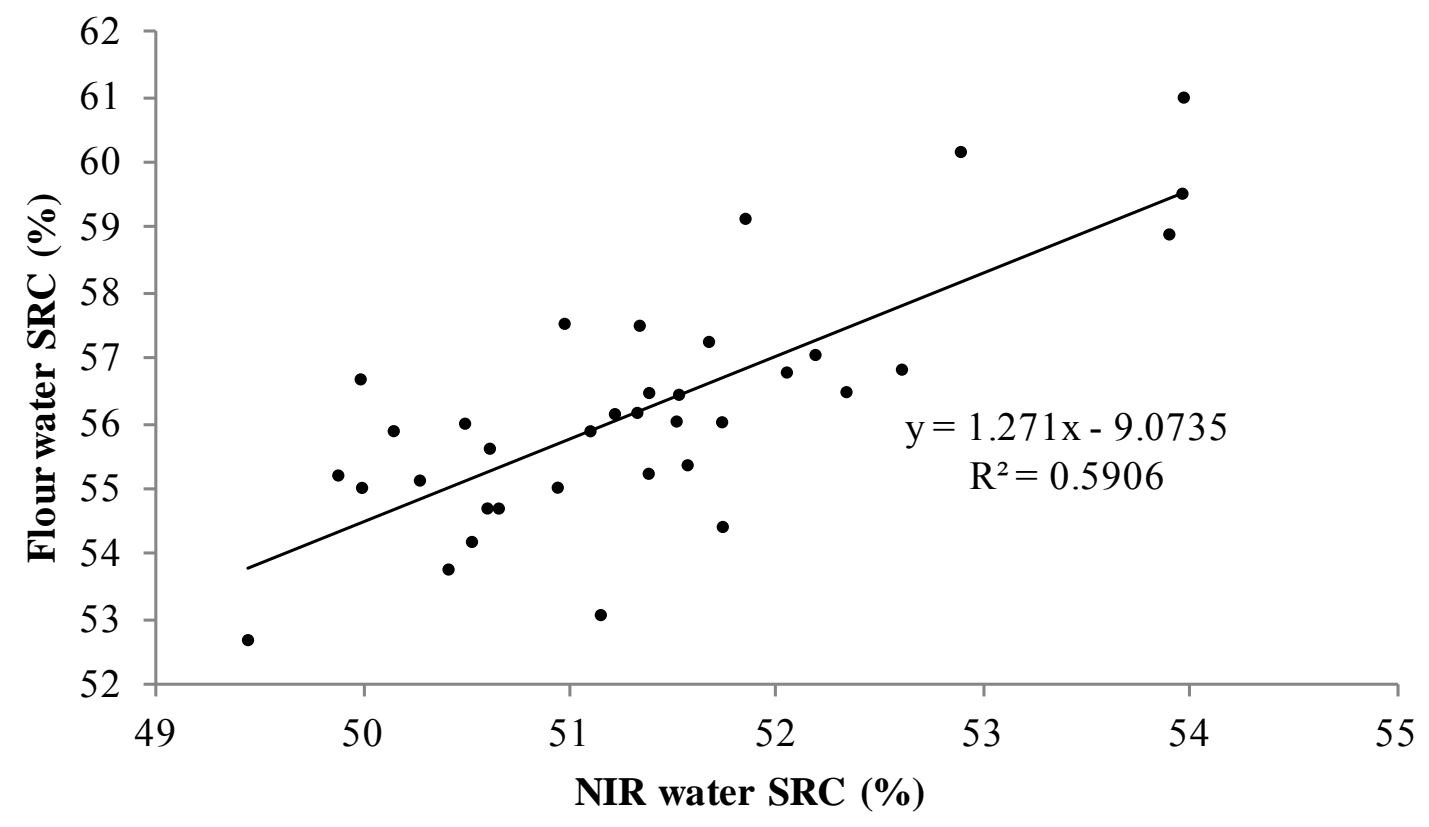


Figure A.4.24: Regression of flour water solvent retention capacity (SRC) on NIR water SRC in population 4 in Lexington 2010 and Princeton 2011.

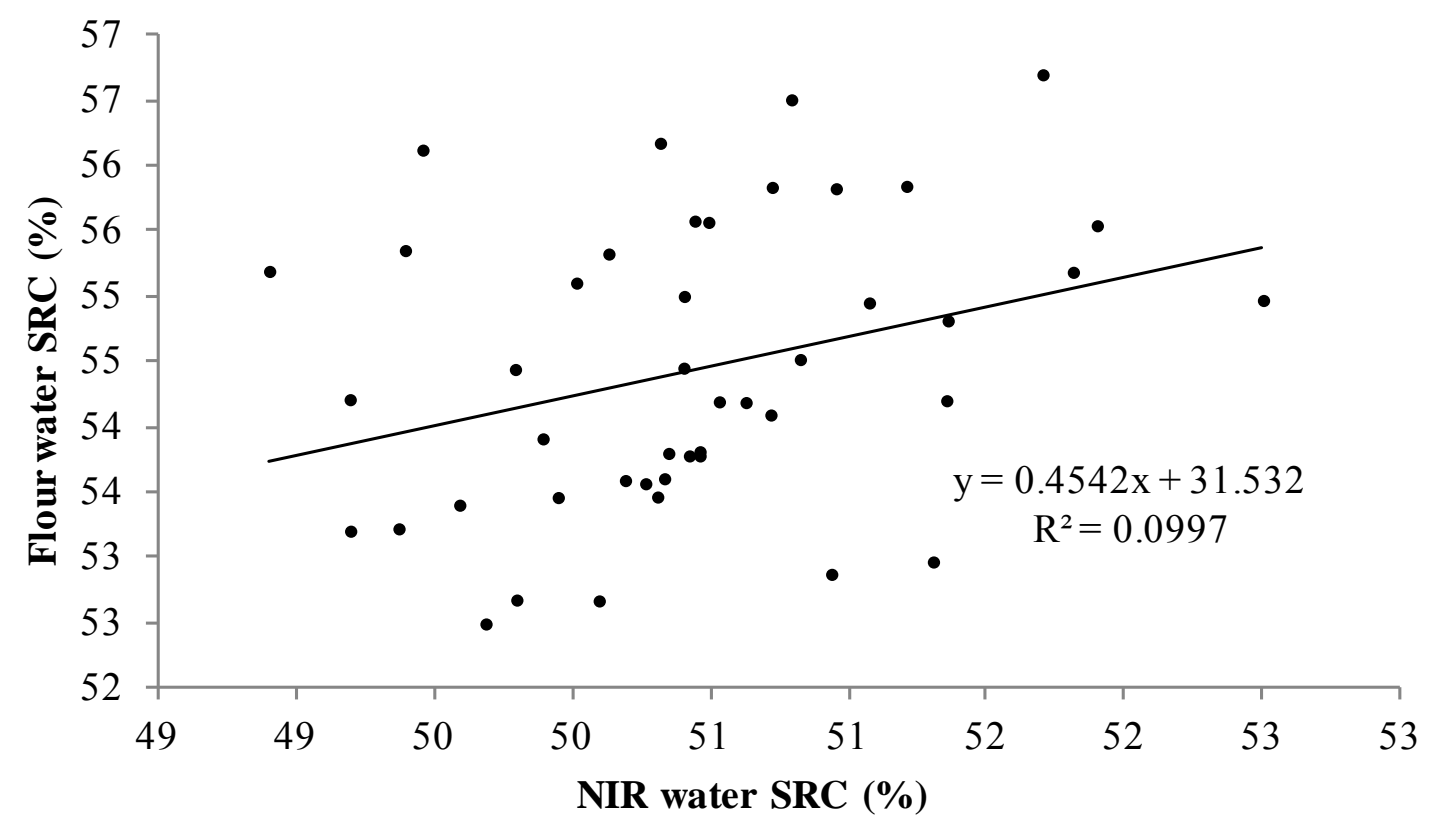


Figure A.4.25: Regression of flour water solvent retention capacity (SRC) on NIR water SRC in population 5 in Lexington 2010 and Princeton 2011.

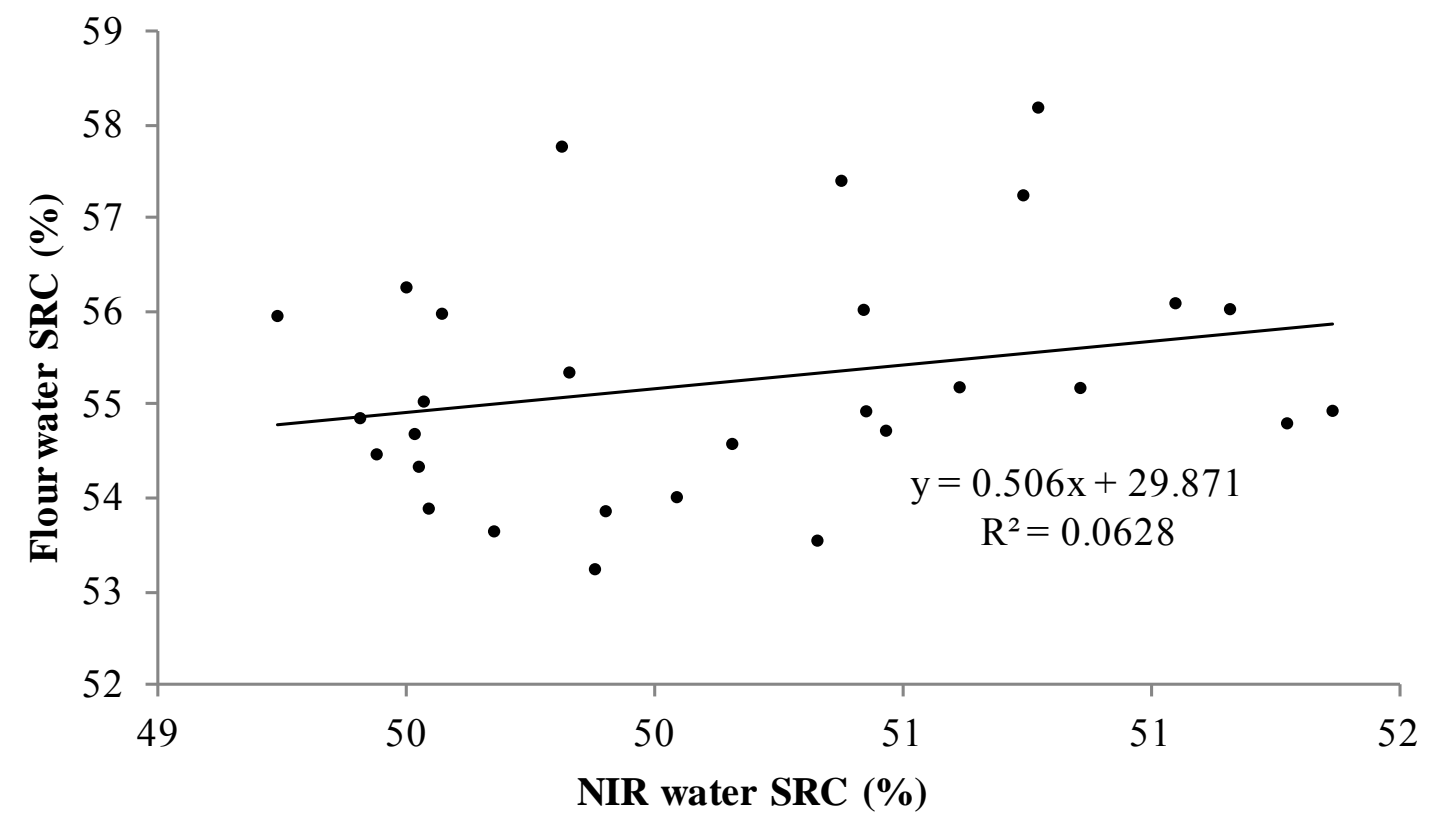


Figure A.5.1: Regression of percentage of Fusarium damaged kernels measured by air separation (FDK) on percentage of Fusarium damaged kernels measured with NIR (NIRFDK) in population 1.

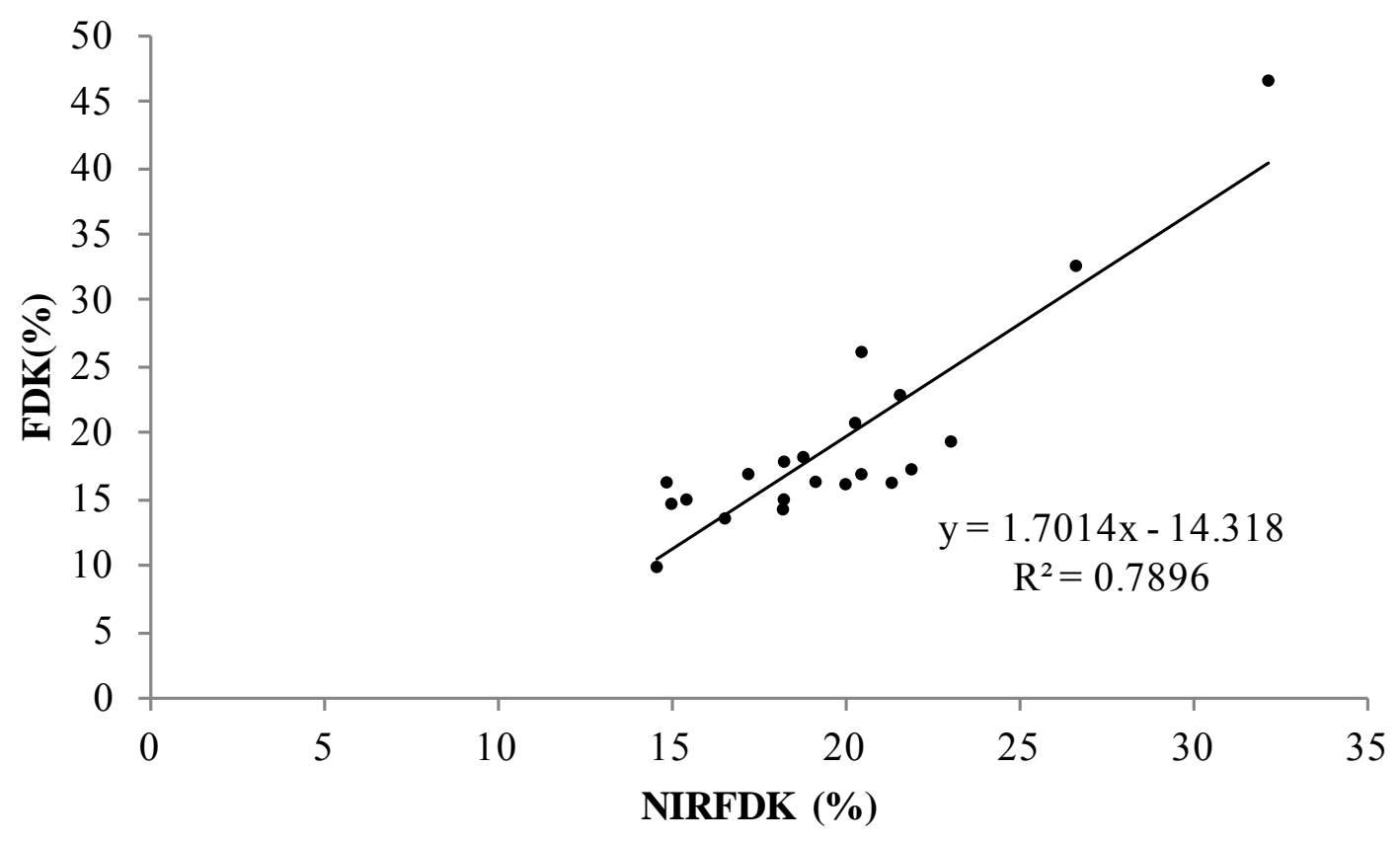


Figure A.5.2: Regression of percentage of Fusarium damaged kernels measured by air separation (FDK) on percentage of Fusarium damaged kernels measured with NIR (NIRFDK) in population 2.

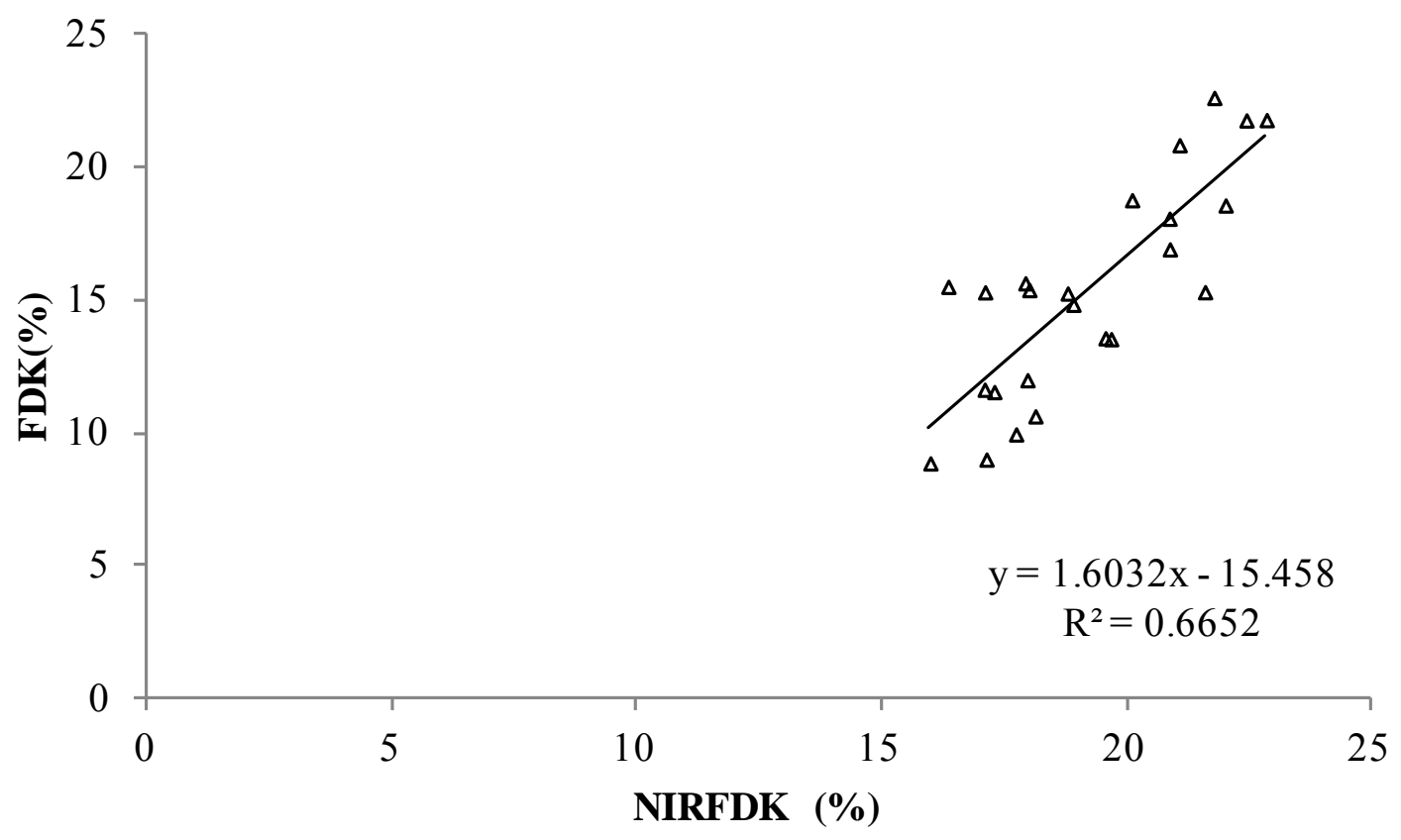


Figure A.5.3: Regression of percentage of Fusarium damaged kernels measured by air separation (FDK) on percentage of Fusarium damaged kernels measured with NIR (NIRFDK) in population 3.

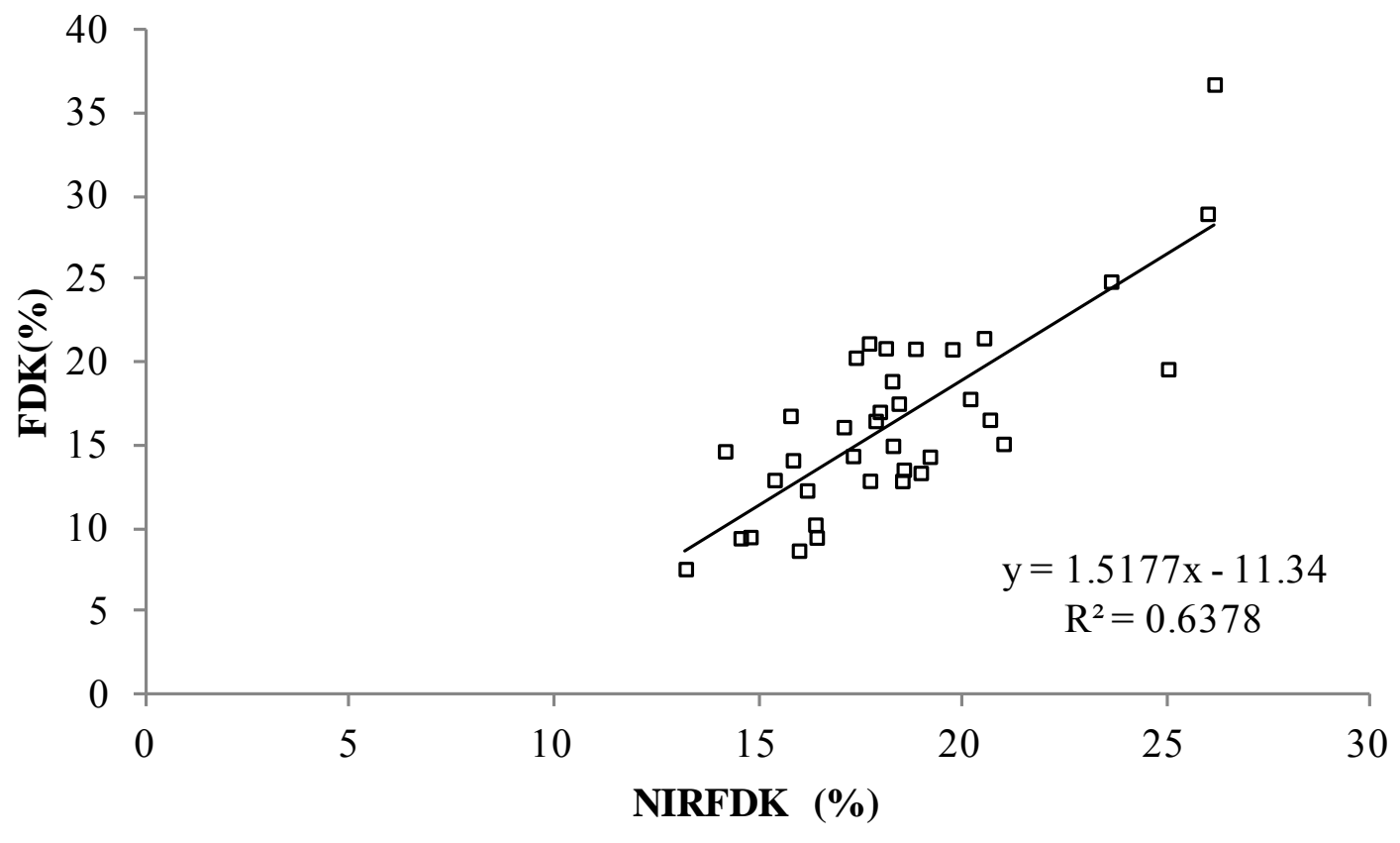


Figure A.5.4: Regression of percentage of Fusarium damaged kernels measured by air separation (FDK) on percentage of Fusarium damaged kernels measured with NIR (NIRFDK) in population 4.

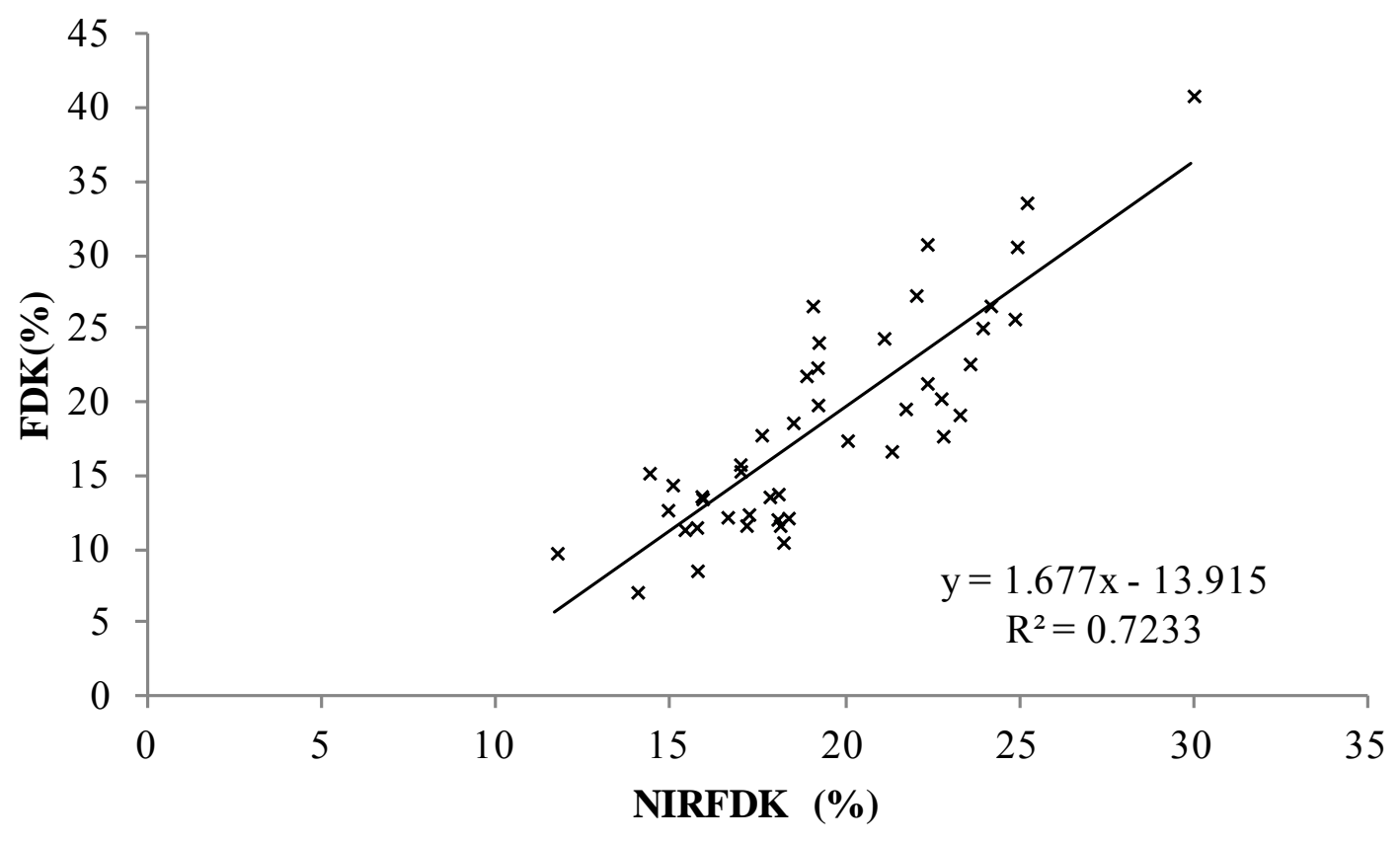


Figure A.5.5: Regression of percentage of Fusarium damaged kernels measured by air separation (FDK) on percentage of Fusarium damaged kernels measured with NIR (NIRFDK) in population 5.

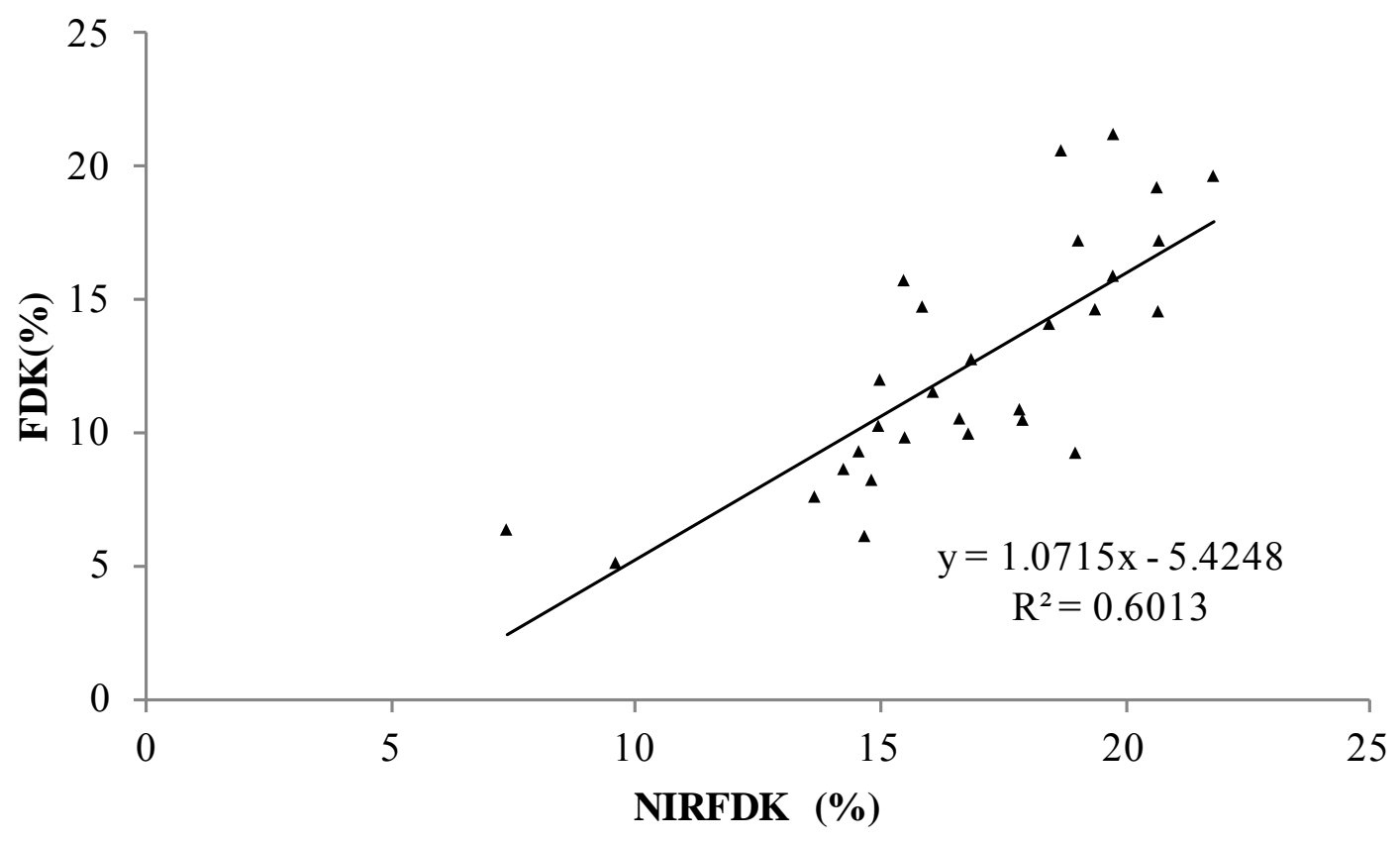


Figure A.5.6: Regression of deoxynivalenol level measured by traditional methods (DON) on deoxynivalenol level measured with NIR (NIRDON) in population 1.

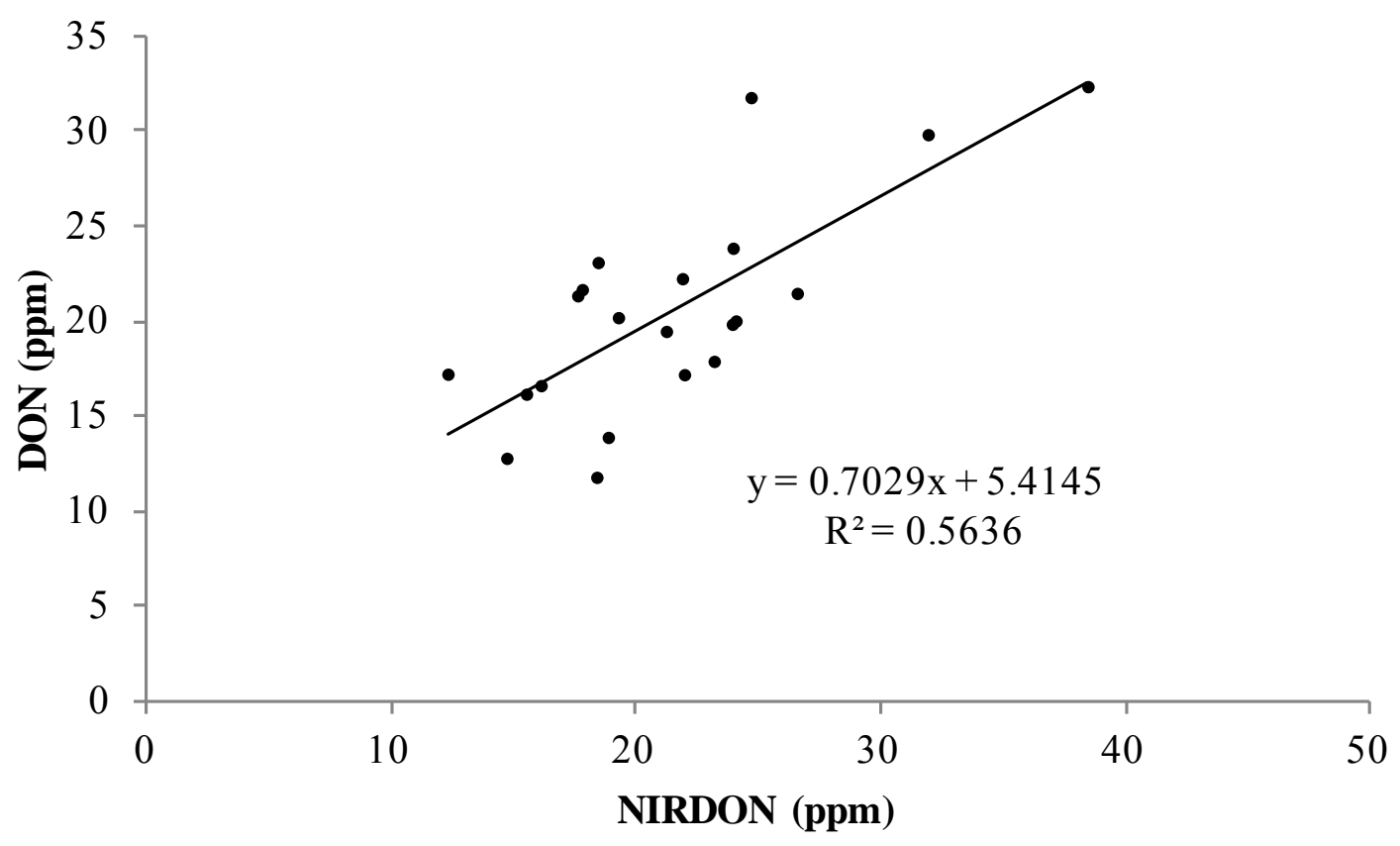


Figure A.5.7: Regression of deoxynivalenol level measured by traditional methods (DON) on deoxynivalenol level measured with NIR (NIRDON) in population 2.

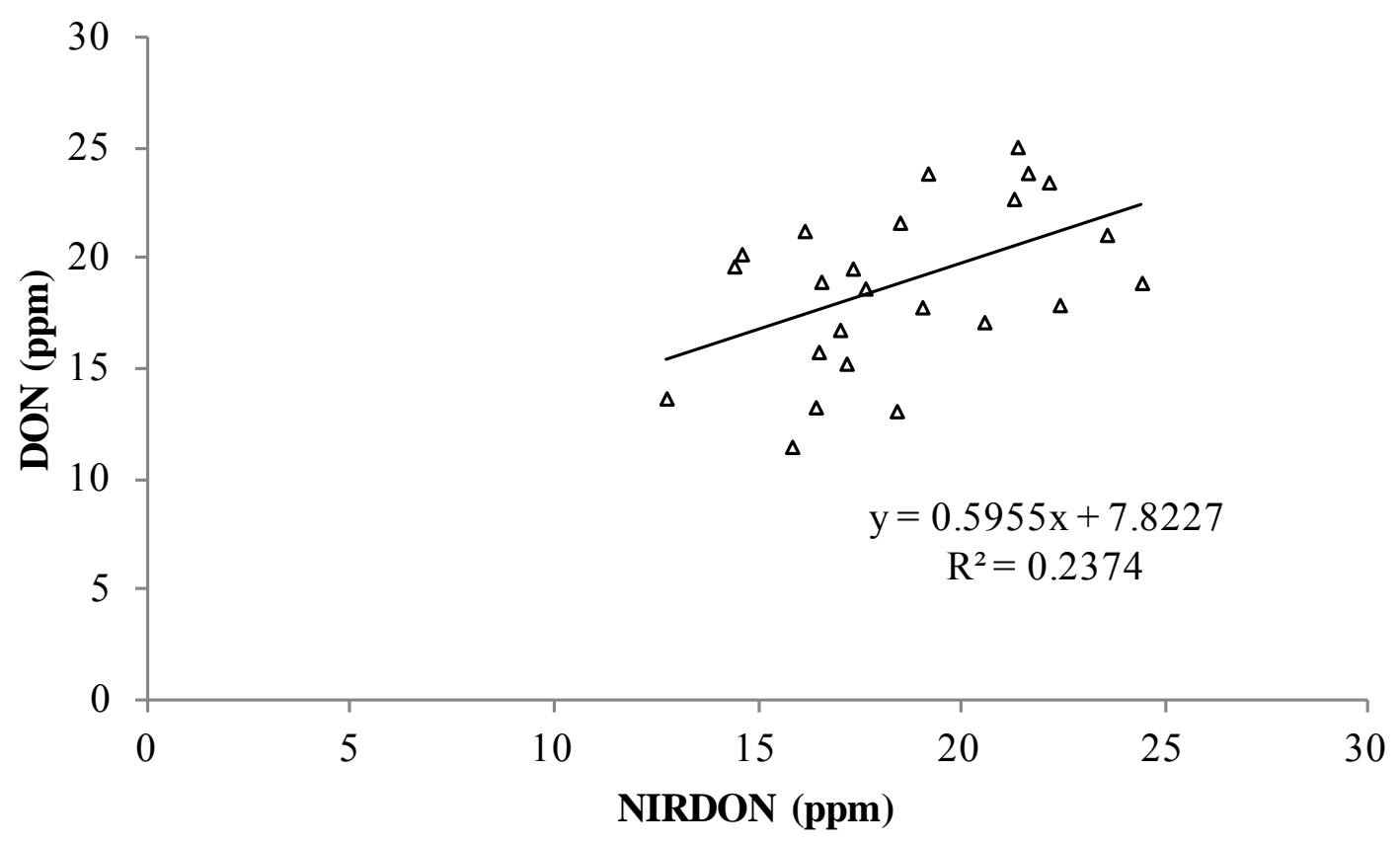


Figure A.5.8: Regression of deoxynivalenol level measured by traditional methods (DON) on deoxynivalenol level measured with NIR (NIRDON) in population 3.

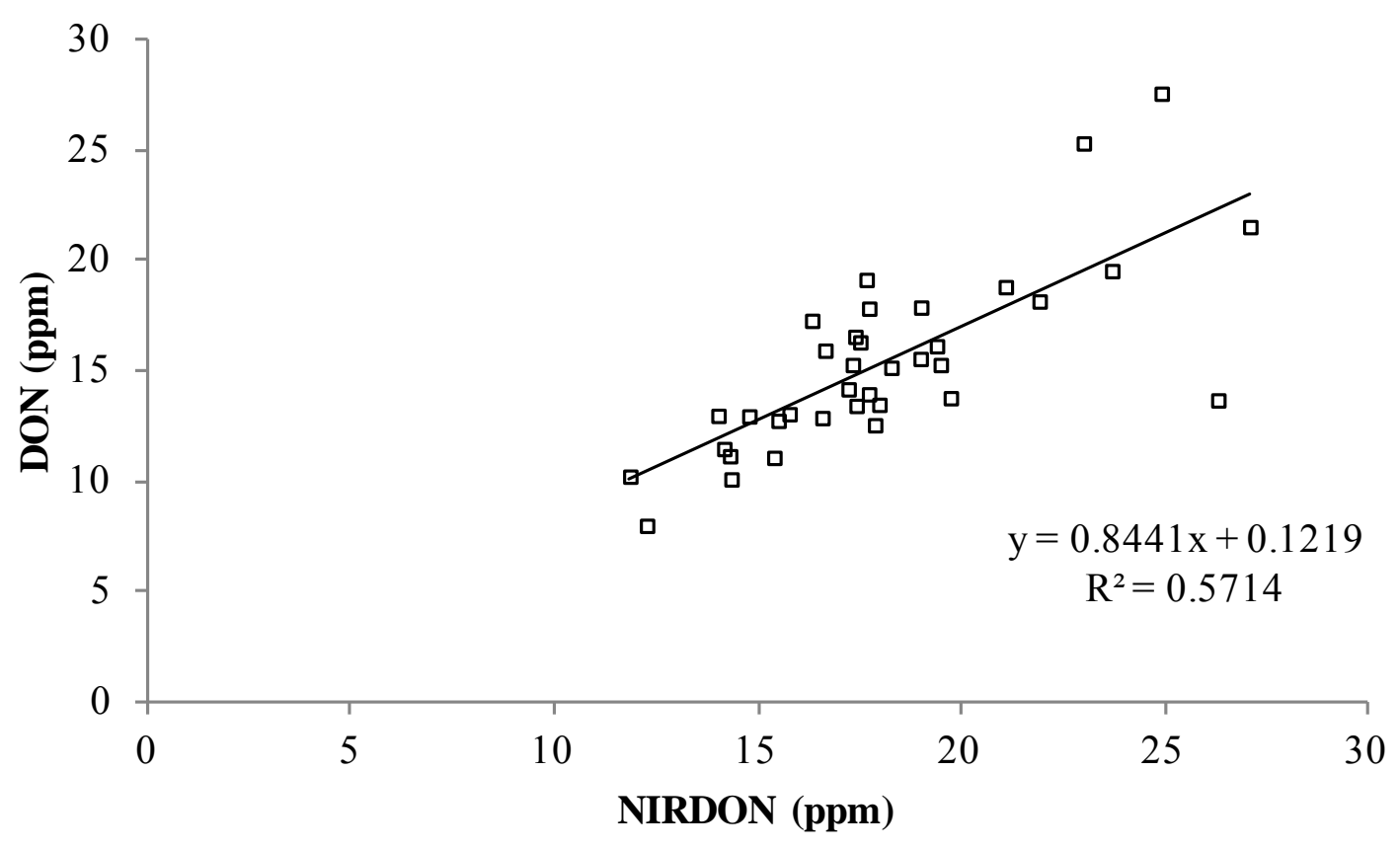


Figure A.5.9: Regression of deoxynivalenol level measured by traditional methods (DON) on deoxynivalenol level measured with NIR (NIRDON) in population 4.

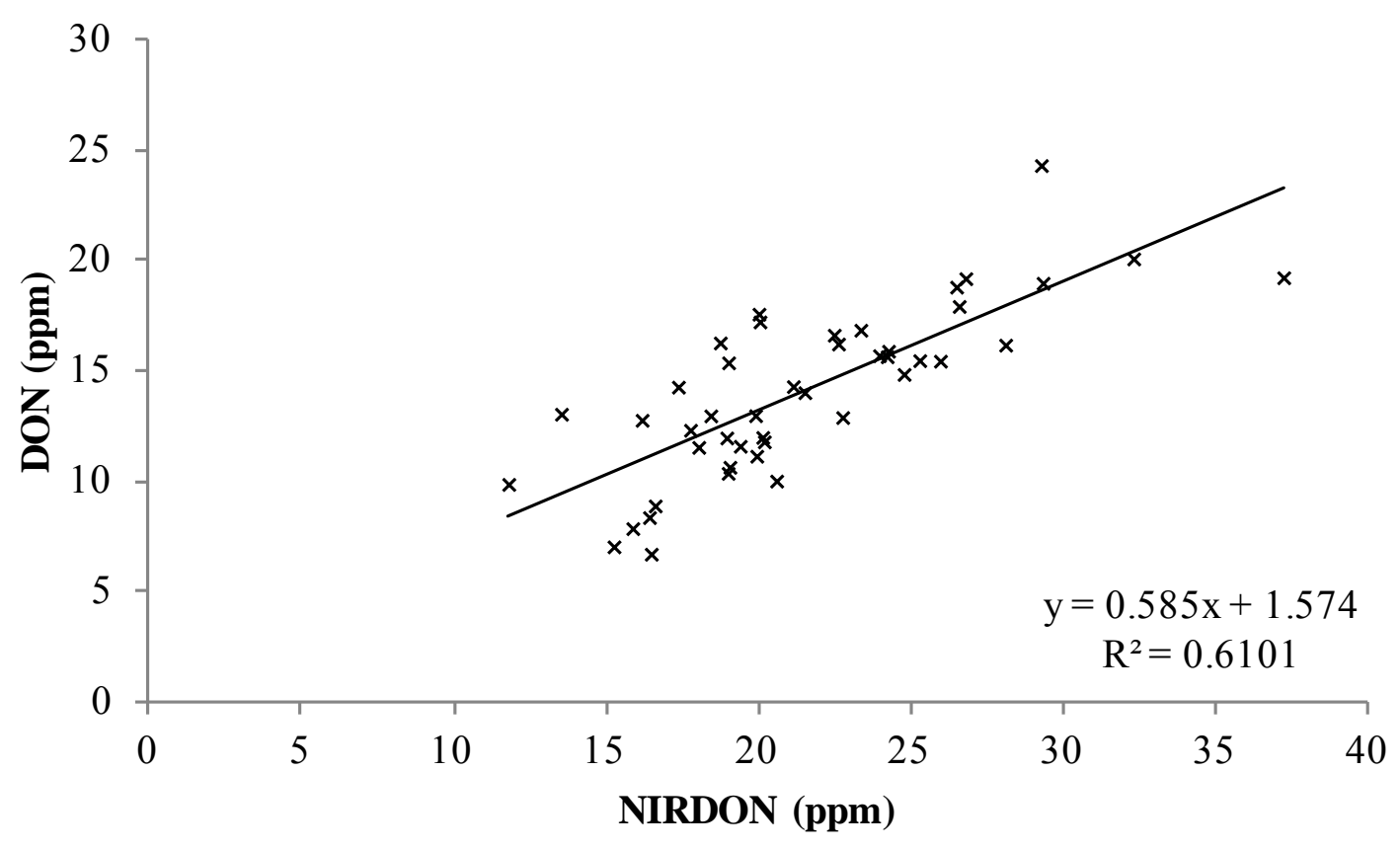


Figure A.5.10: Regression of deoxynivalenol level measured by traditional methods (DON) on deoxynivalenol level measured with NIR (NIRDON) in population 5.

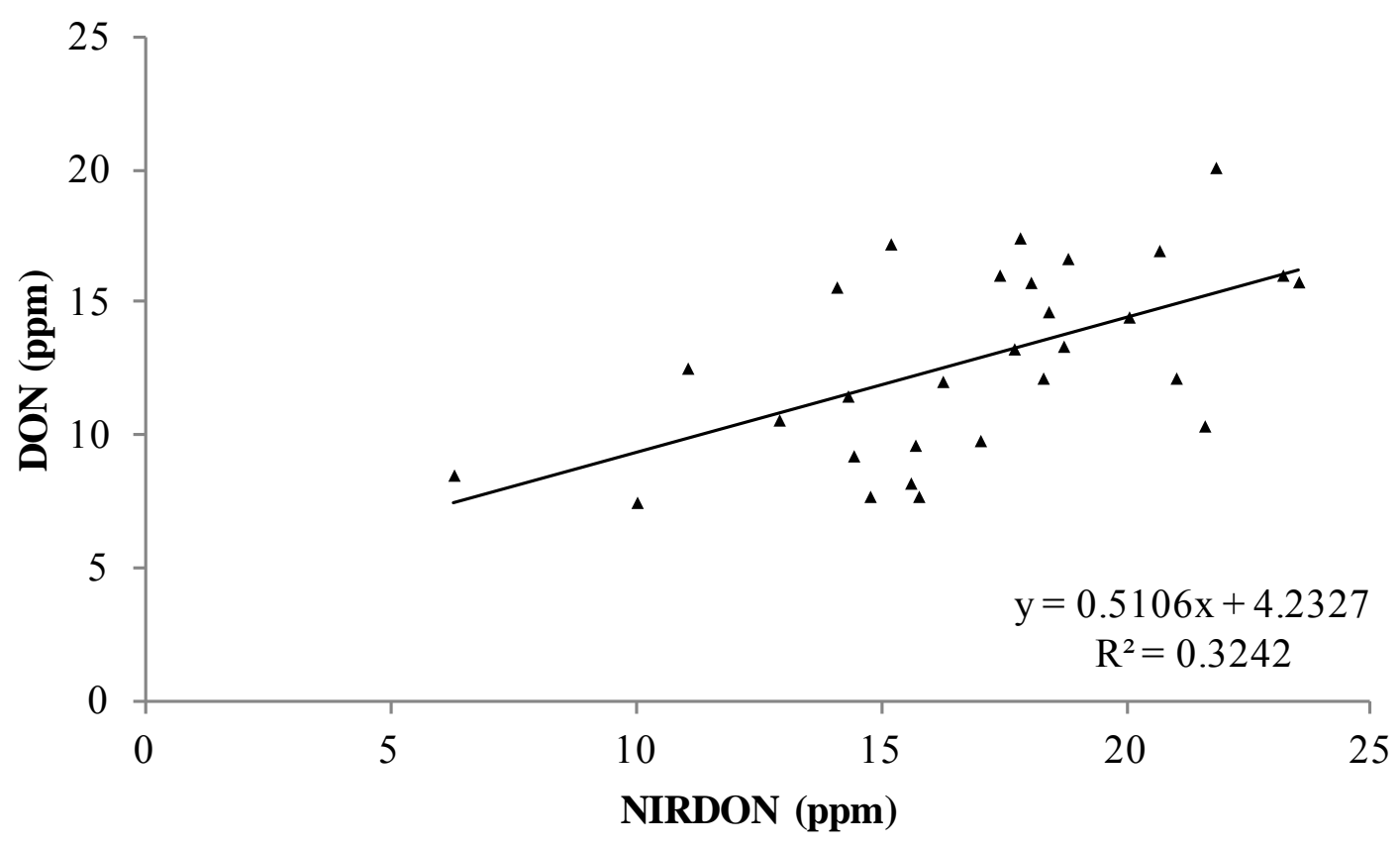


Figure A.5.11: Regression of deoxynivalenol level measured by traditional methods (DON) on percentage of fusarium damaged kernels measured with NIR (NIRFDK) in population 1.

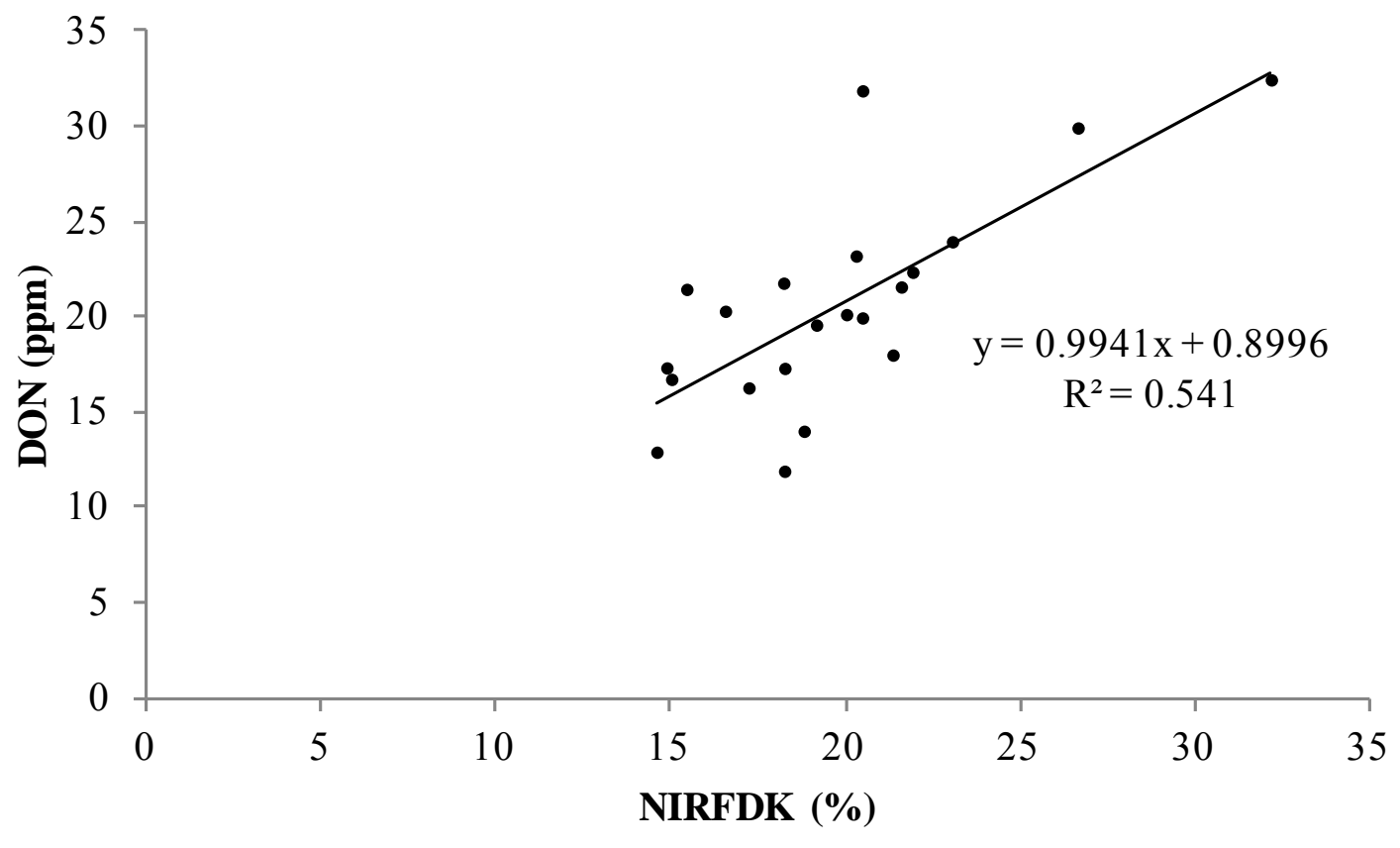


Figure A.5.12: Regression of deoxynivalenol level measured by traditional methods (DON) on percentage of Fusarium damaged kernels measured by air separation (FDK) in population 1 .

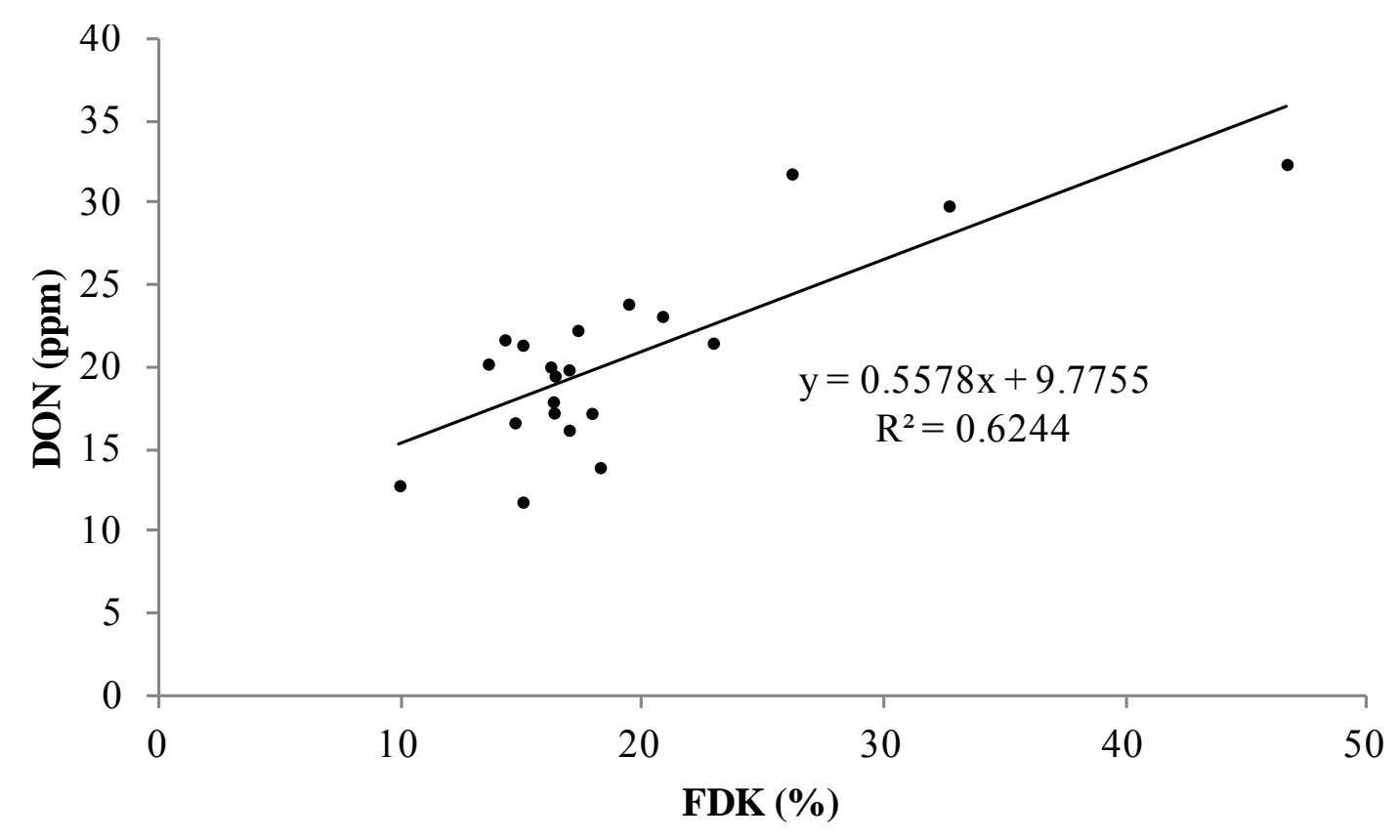


Figure A.5.13: Regression of deoxynivalenol level measured by traditional methods (DON) on percentage of Fusarium damaged kernels measured with NIR (NIRFDK) in population 2.

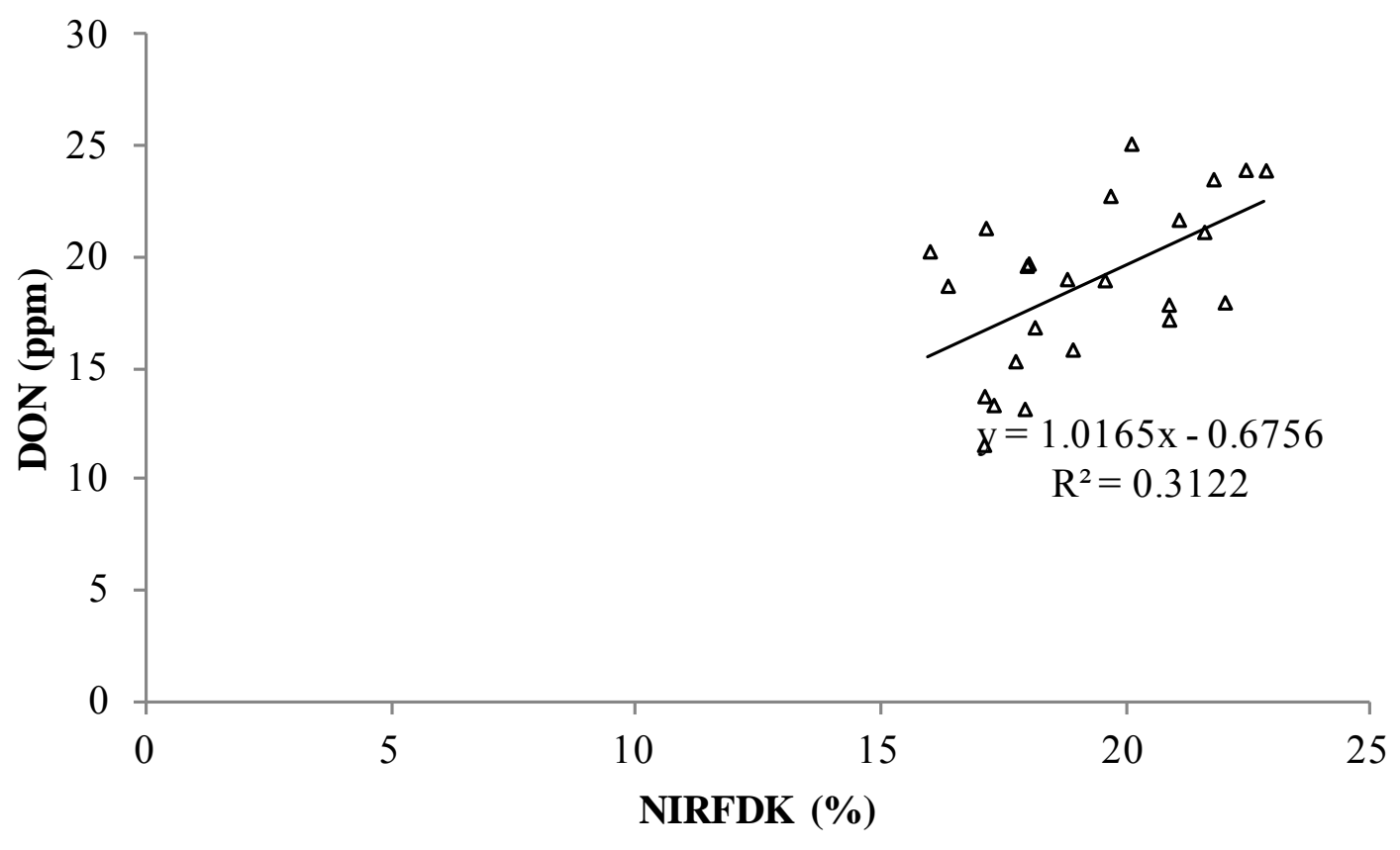


Figure A.5.14: Regression of deoxynivalenol level measured by traditional methods (DON) on percentage of Fusarium damaged kernels measured by air separation (FDK) in population 2 .

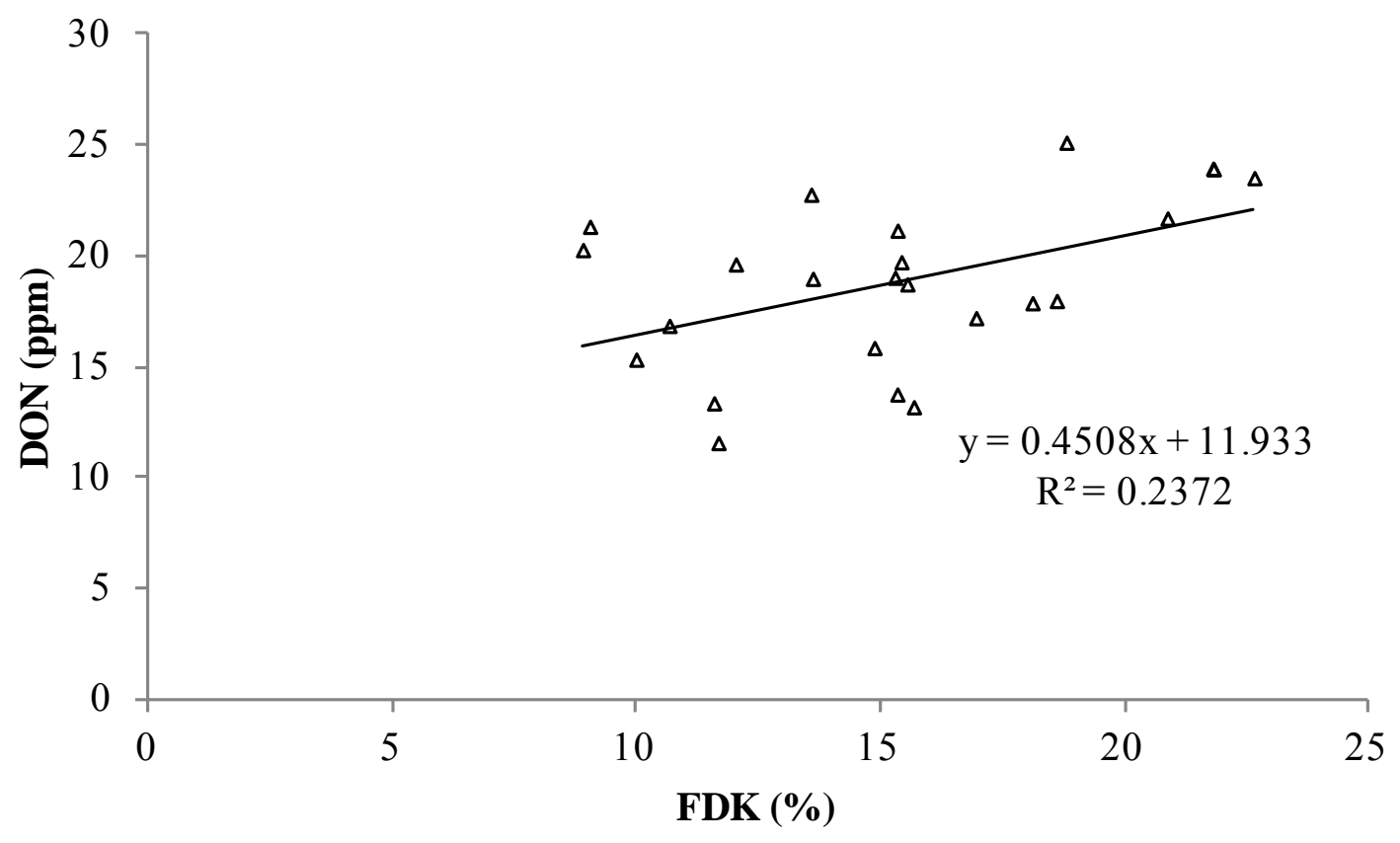


Figure A.5.15: Regression of deoxynivalenol level measured by traditional methods (DON) on percentage of Fusarium damaged kernels measured with NIR (NIRFDK) in population 3.

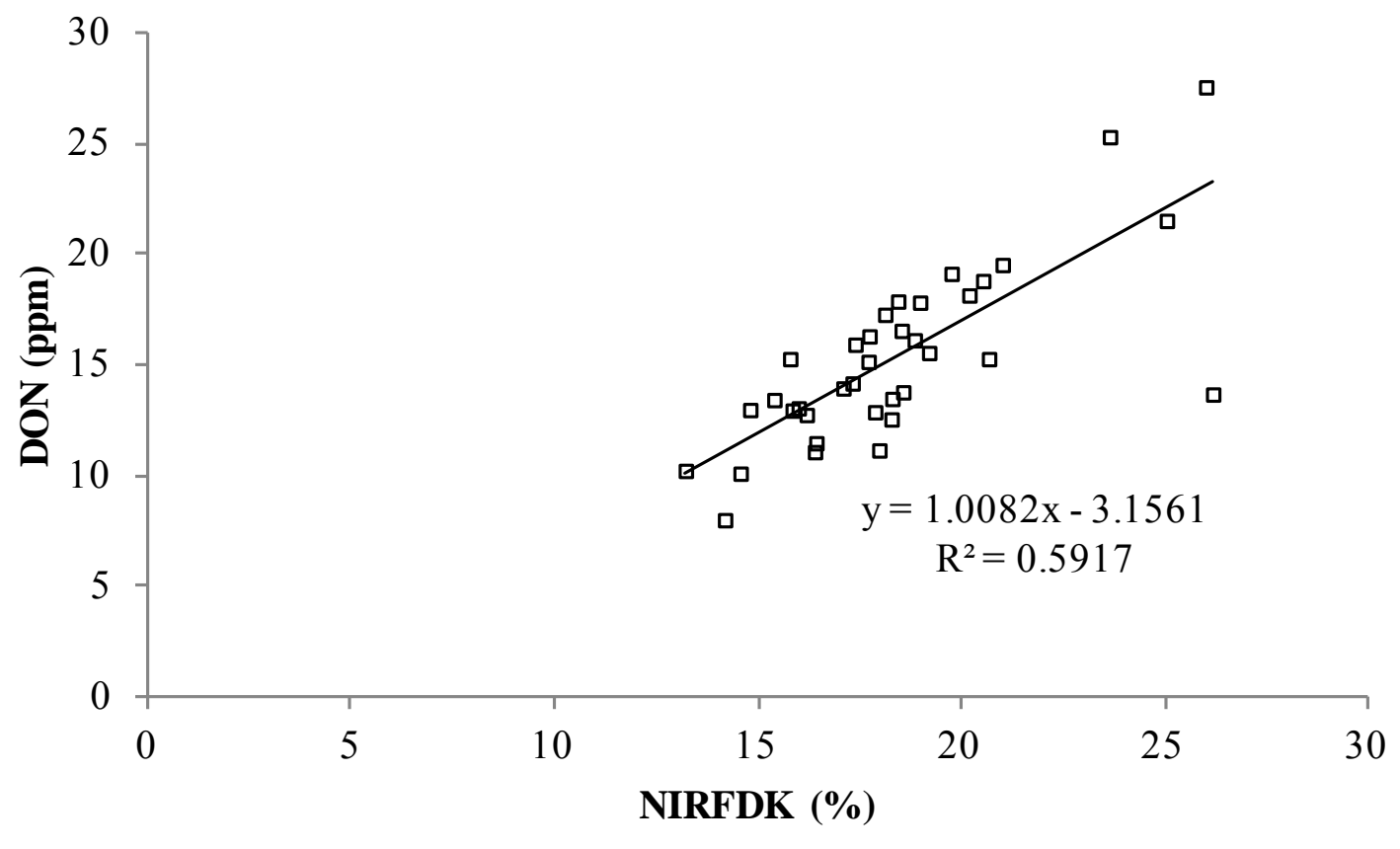


Figure A.5.16: Regression of deoxynivalenol level measured with traditional methods (DON) on percentage of Fusarium damaged kernels measured by air separation (FDK) in population 3 .

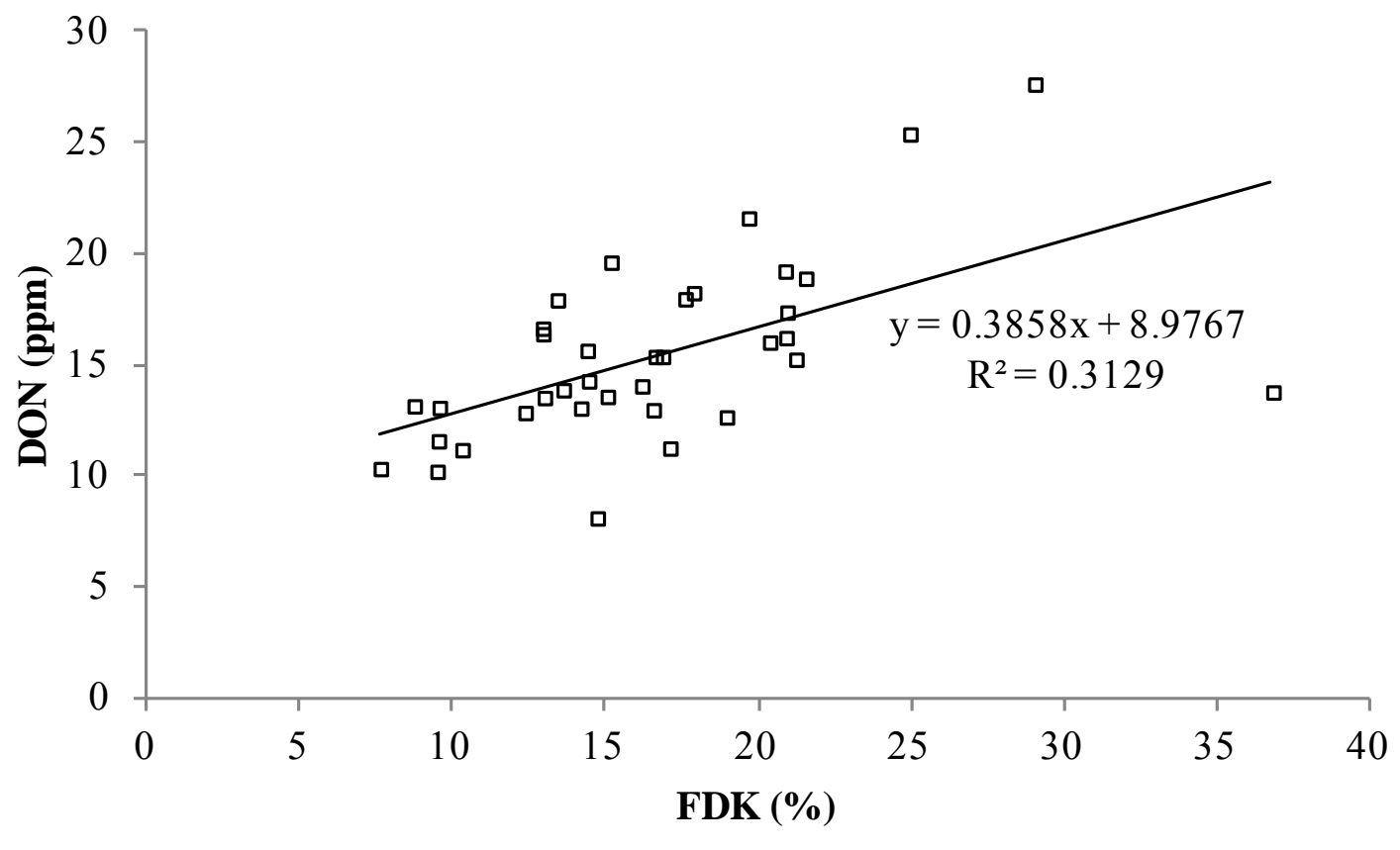


Figure A.5.17: Regression of deoxynivalenol level measured with traditional methods (DON) on percentage of Fusarium damaged kernels measured with NIR (NIRFDK) in population 4.

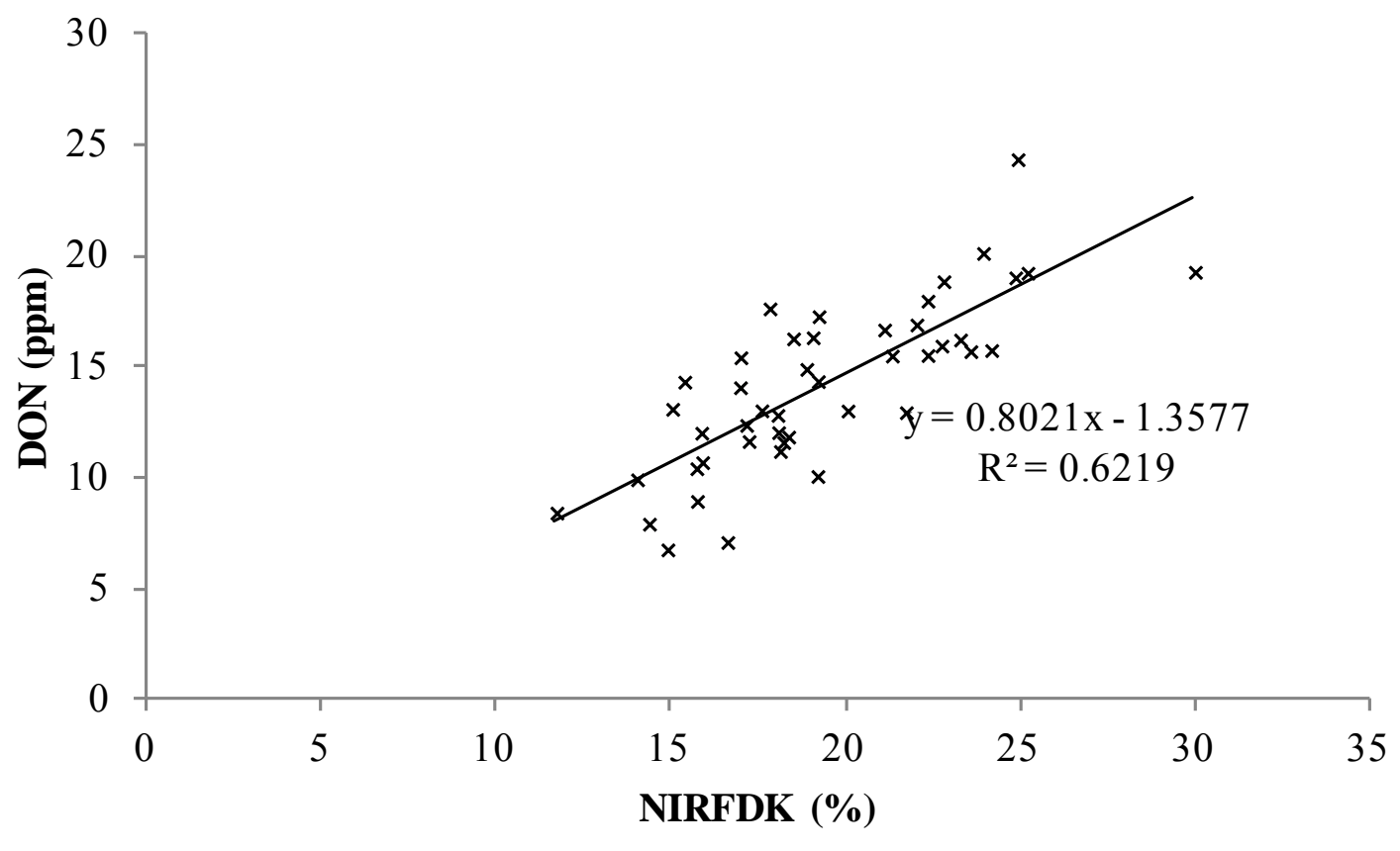


Figure A.5.18: Regression of deoxynivalenol level measured with traditional methods (DON) on percentage of Fusarium damaged kernels measured by air separation (FDK) in population 4 .

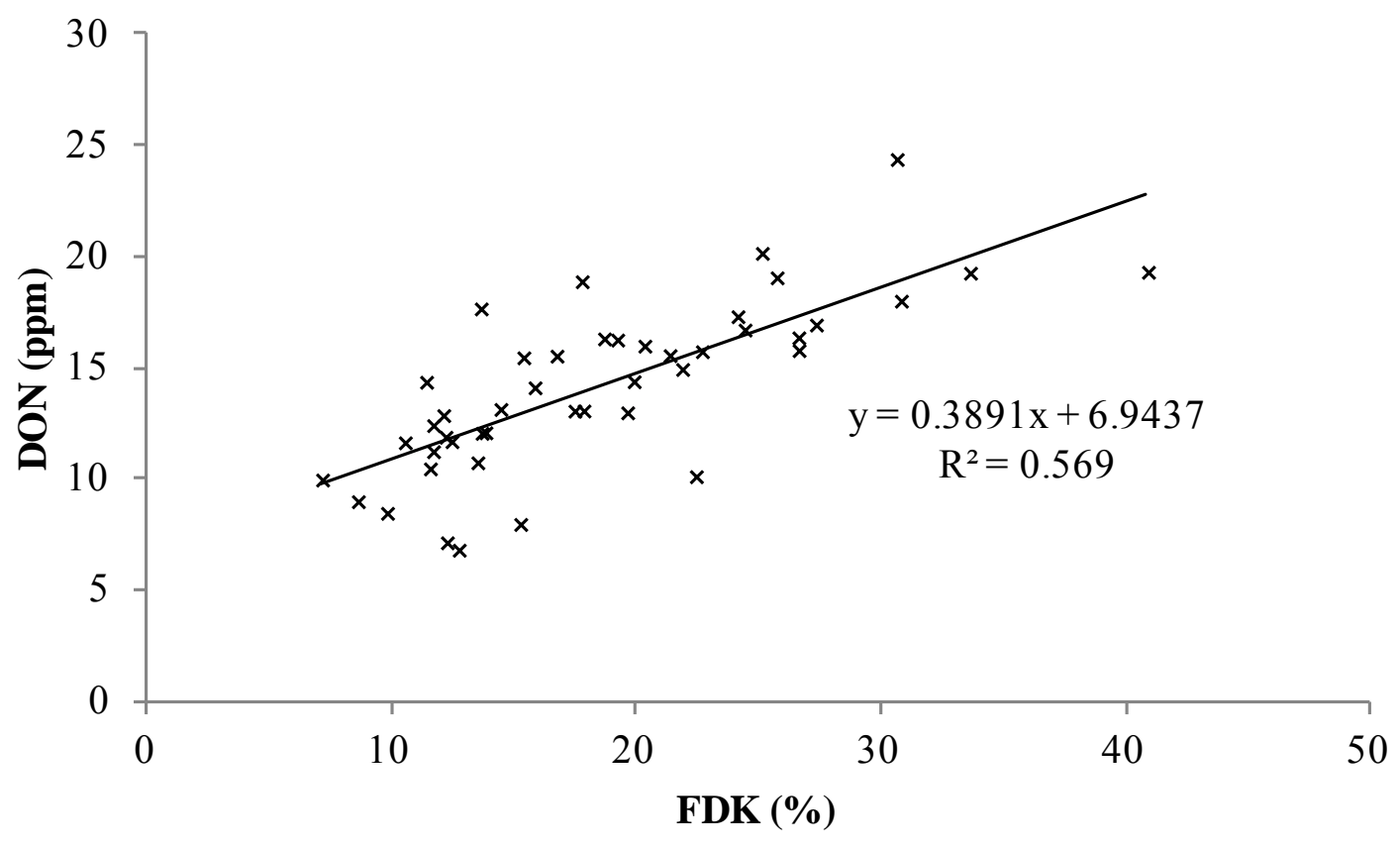


Figure A.5.19: Regression of deoxynivalenol level measured with traditional methods (DON) on percentage of Fusarium damaged kernels measured with NIR (NIRFDK) in population 5 .

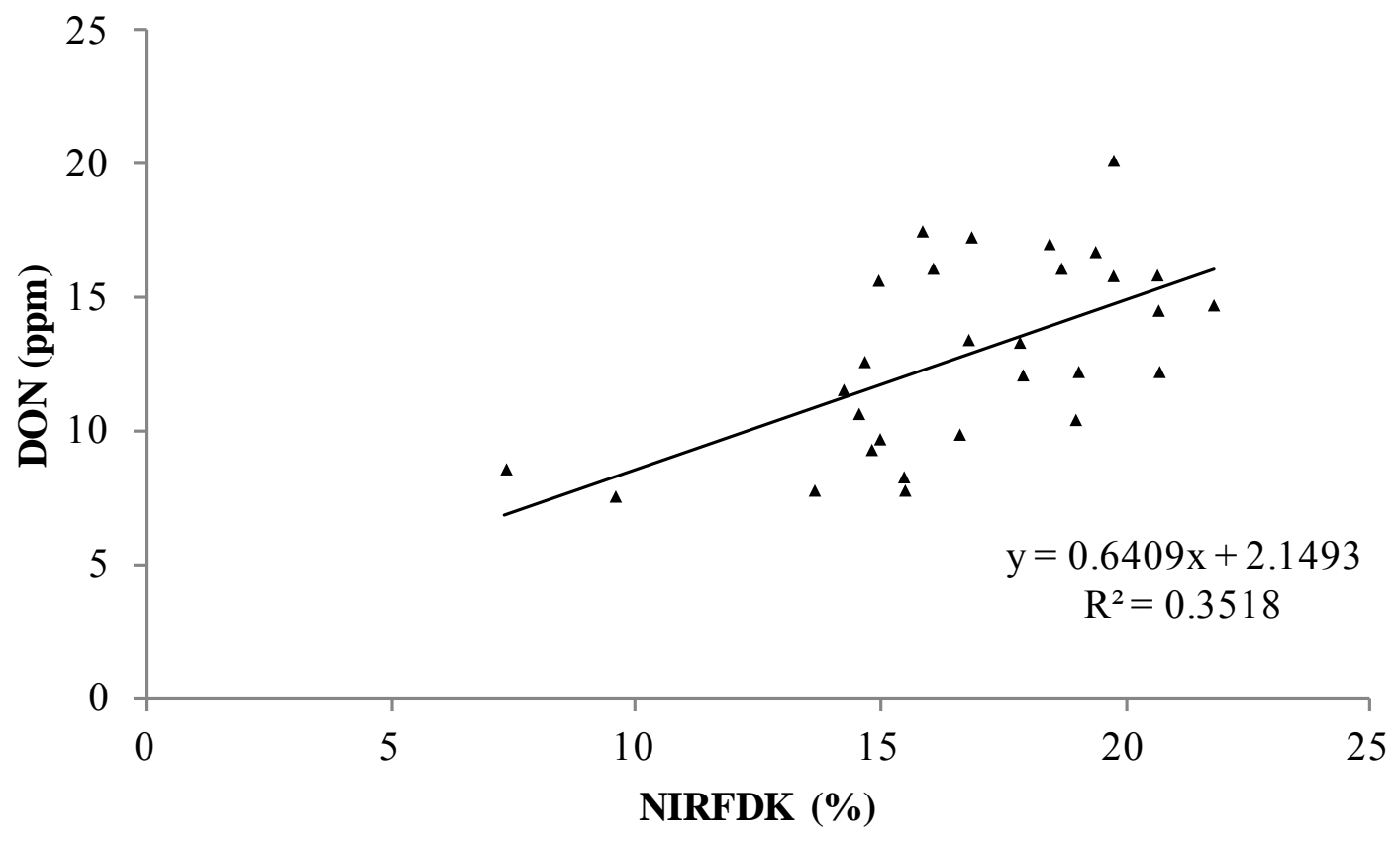


Figure A.5.20: Regression of deoxinivalenol level measured with traditional methods (DON) on percentage of Fusarium damaged kernels measured by air separation (FDK) in population 5 .

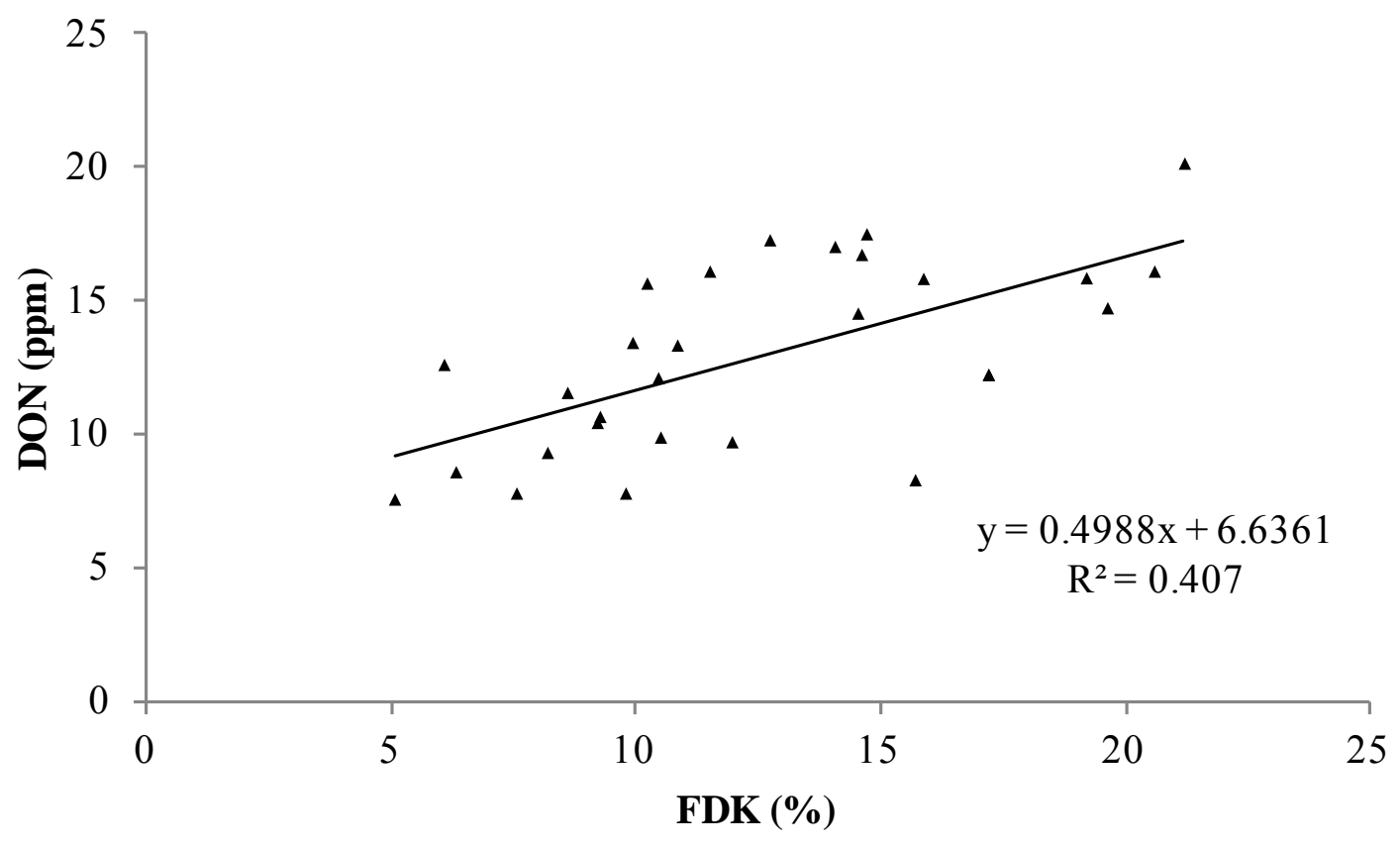




\section{REFERENCES}

American Association of Cereal Chemists (AACC). 2010. Approved methods of the AACC, 10th ed. American Association of Cereal Chemists, St. Paul, MN.

American Association of Cereal Chemists (AACC) International. 2011. Approved methods of analysis, 11 th ed. AACC International, St. Paul, MN. Available at http://methods.aaccnet.org/toc.aspx

Anonymous, 2005: Commission Regulation (EC) No 856/2005 of 6 June 2005 amending regulation (EC) no 466/2001 as regards Fusarium toxins. Official Journal of the European Union. Available at http://eurlex.europa.eu/LexUriServ/LexUriServ.do?uri=OJ:L:2005:143:0003:0008:EN:PD $\underline{F}$

Agostinelli, A. M., Clark, A. J., Brown-Guedira, G., Van Sanford, D. A. 2012. Optimizing phenotypic and genotypic selection for Fusarium head blight resistance in wheat. Euphytica 186: 115-126.

Agostinelli, A., Mundell, N., Van Sanford, D. 2008. Percentage of Fusarium damaged kernels measured by air separation. In: Canty, S., Clark, A., Walton, E., Ellis, D., Mundell, J., Van Sanford, D. (eds) Proceedings of the 2008 National Fusarium head blight forum, December 2-4, Indianapolis, IN.

Argyris, J. M., TeKrony, D. M., Van Sanford, D. 2001. Effect of Fusarium graminearum infection during seed development on seed quality. Proceedings of the 2001 National Fusarium head blight forum, December 8-10, Erlanger, KY.

Bai, G., Shaner, G. 1994. Scab of Wheat: Prospects for Control. Plant Dis. 78:760-766. 
Bai, G., Shaner, G. 1996. Variation in Fusarium graminearum and cultivar resistance to scab. Plant Dis. 80:975-979.

Bai, G., Shaner, G. 2004. Management and Resistance in Wheat and Barley to Fusarium Head Blight. Annu. Rev. Phytopathol. 42:135- 161.

Bai, G., Shaner, G., Ohm, H., 2000. Inheritance of resistance to Fusarium graminearum in wheat. Theor. Appl. Genet. 100:1-8.

Bernardo, R. 2002. Breeding for quantitative traits in plants. Stemma Press, Woodbury, MN, pp 369.

Beuerlein, J. 2001. Classes and uses of wheat. Ohio State University Extension Fact Sheet. Horticulture and Crop Science AGF 146-0, Columbus, OH. Available at http://ohioline.osu.edu/agf-fact/0146.html

Buerstmayr, H., Lemmens, M., Hartl, L., Doldi, L., Steiner, B., Stierschneider, M., Ruckenbauer, P. 2002. Molecular mapping of QTLs for Fusarium head blight resistance in spring wheat. I. Resistance to fungal spread (Type II resistance). Theor. Appl. Genet. 104: 84-91.

Buerstmayr, H., Steiner, B., Hartl, L., Griesser, M., Angerer, N., Lengauer, D., Miedaner, T., Schneider, B., Lemmens, M. 2003. Molecular mapping of QTLs for Fusarium head blight resistance in spring wheat. II. Resistance to fungal penetration and spread. Theor. Appl. Genet. 107:503-508.

Buerstmayr, H., Ban, T., Anderson, J. A. 2009. QTL mapping and marker-assisted selection for Fusarium head blight resistance in wheat: a review. Plant Breed. 128:1- 26. 
Buerstmayr, H., Steiner, B., Lemmens, M. 2010. Current knowledge on the genetics of Fusarium head blight resistance in wheat- Implications for resistance breeding. In: Canty, S., Clark, A., Anderson-Scully, A., Ellis, E., Van Sanford, D. (eds) Proceedings of the 2010 National Fusarium Head Blight Forum, December 7-9, Milwaukee, WI.

Cardwell, L. A. 2011. Scab resistance QTLs are associated with quality and agronomic traits of soft red winter wheat. Dissertation, University of Maryland, College Park, MD. Available at http://hdl.handle.net/1903/11481

Cowger, C., and Sutton, A. L. 2005. The southeastern U.S. Fusarium head blight epidemic of 2003. Plant Health Progress. Available at http://www.plantmanagementnetwork.org/pub/php/research/2005/fhb/

Delwiche, S. R., Hareland, G. A. 2004. Detection of scab-damaged hard red spring wheat kernels by near-infrared reflectance. Cereal Chem. 81: 643-649.

Delwiche, S. R., Kim, M. S., Dong, Y. 2011. Fusarium damage assessment in wheat kernels by Vis/NIR hyperspectral imaging. Sens. \& Instrumen. Food Qual. 5:6371.

Dufault, N., De Wolf, E., Lipps, P., Madden, L. 2002. Identification of environmental variables that affect perithicial development of Gibberella zeae. In: Canty, S., Lewis, J., Siler, L., Ward, R. (eds) Proceedings of the 2002 National Fusarium head blight forum, December 7-9, Erlanger, KY.

Dufault, N., De Wolf, E., Lipps, P., Madden, L. 2002. Relationship of temperature and moisture to Gibberella zeae perithecial development in a controlled environment. In: Canty, S., Lewis, J., Siler, L., Ward, R. (eds) Proceedings of the 2002 National Fusarium head blight forum, December 7-9, Erlanger, KY. 
Everts, K.L., Leath, S., Finney, P. L. 2001. Impact of powdery mildew and leaf rust on milling and baking quality of soft red winter wheat. Plant Dis. 85: 423-429.

Food and Drug Advisory (FDA). 2010. Guidance for industry and FDA: Advisory levels for deoxynivalenol (DON) in finished wheat products for human consumption and grains and grain by-products used for animal feed. Available at http://www.fda.gov/Food/GuidanceComplianceRegulatoryInformation/Guidance Documents/NaturalToxins/ucm120184.htm

Gale, L. R. 2003. Population biology of Fusarium species causing head blight of grain crops. In: Leonard, K. J., Bushnell, W. R. (eds) Fusarium head blight of wheat and barley, APS Press, St. Paul, MN, pp 120-143

Gooding, R. W., Lafever, H. N., Campbell, K. G., Herald, L. D. 1997. Registration of 'Freedom' wheat. Crop Sci. 37:1007.

Griffey, C. A., Starlin, T. M., Price, A. M., Sisson, W. L., Das, M. K., Pridgen, T. H., Vaughn, M. E., Rohrer, W. L., Brann, D. E. 2001. Registration of 'Roane' wheat. Crop Sci. 41: 1359-1360.

Guttieri, M. J., Bowen, D., Gannon, D., O’Brien, K., Souza, E. 2001. Solvent retention capacities of irrigated soft white spring wheat flours. Crop Sci. 41:1054-1061.

Guttierri, M. J., Becker, C., Souza, E. J. 2004. Application of wheat meal solvent retention capacity tests within soft wheat breeding programs. Cereal Chem. 81:261-266.

Hershman, D. E, Johnson, D. W. 2009. Disease management. In: ID 125 A comprehensive guide to wheat management in Kentucky, Cooperative Extension Service, University of Kentucky, College of Agriculture, Lexington, KY, pp 4254. 
Institute SAS. 2002. The SAS System for Windows, release 9.1. SAS Institute Inc, Cary.

Johnson, D.D., Flaskerud, G. K., Taylor, R. D., Satyanarayana, V. 2003. Quantifying economic impacts of Fusarium head blight in wheat. In: Leonard, K. J., Bushnell, W. R. (eds) Fusarium head blight of wheat and barley, APS Press, St. Paul, MN, pp 461-483.

Kang, J., Clark, A., Van Sanford, D., Griffey, C., Brown-Guedira, G., Dong, Y., Murphy, J. P., Costa, J. 2011. Exotic scab resistance quantitative trait loci effects on soft red winter wheat. Crop Sci. 51:924-933.

Knapp, S. J., Stroup, W. W., Ross, W. M. 1985. Exact confidence intervals for heritability on a progeny mean basis. Crop Sci. 25:192-195

Knott, C. A.2007. Breeding for value-added traits in soft winter wheat. Dissertation, University of Kentucky, Lexington, USA.

Knott, C. A., Van Sanford, D. A., Souza, E. J. 2009. Genetic variation and the effectiveness of early-generation selection for soft winter wheat quality and gluten strength. Crop Sci. 49:113-119.

Kweon, M., Slade, L., Levine, H. 2011. Solvent retention capacity (SRC) testing of wheat flour: principles and value in predicting flour functionality in different wheatbased food processes in wheat breeding- A review. Cereal Chem. 88: 537-552.

Lee, C., Herbek, J., Van Sanford, D., Bruening, W. 2009. Cultural practices. In: ID 125 A comprehensive guide to wheat management in Kentucky, Cooperative Extension Service, University of Kentucky, College of Agriculture, Lexington, KY, pp 1319. 
McCartney, C. A., Somers, D.J., Fedak, G., DePauw, R. M., Thomas, J., Fox, S. L., Humphreys, D. G., Lukow, O., Savard, M. E., McCallum, B. D., Gilbert, J., Cao, W. 2007. The evaluation of FHB resistance QTLs introgressed into elite Canadian spring wheat germplasm. Mol. Breed. 20:209-221.

McKendry, A. L., Berg, J. E., Tague, D. N., Kephart, K. D. 1995. Registration of 'Ernie’ wheat. Crop Sci. 35:1513.

McKendry, A. L., Tague, D. N., Wright, R. L., Tremain, J. A., Conley, S. P. 2005. Registration of 'Truman' wheat. Crop Sci. 45:421-423.

McMullen, M., R. Jones and D. Gallenberg. 1997. Scab of wheat and Barley: A Reemerging Disease of Devastating Impact. Plant Dis. 81:1340-1348.

McMullen, M., Shaobin, Z., Neate, S. 2008. Fusarium head blight (scab) of small grains. Plant Disease Management NSDU Extension Service. PP-804 (Revised). Available at http://www.ag.ndsu.edu/pubs/plantsci/smgrains/pp804.pdf

Mesterhazy, A., Bartok, T., Mirocha, C. G., Komoroczy, R. 1999. Nature of wheat resistance to Fusarium head blight and the role of deoxynivalenol for breeding. Plant Breed. 118:97-110.

Microsoft Excel. 2007. Microsoft Office Excel 2007. Microsoft Corp. Redmond, WA.

Mirocha, C.J., Kolaczkowski, E., Xie, W., Yu, H., Jelen, H. 1998. Analysis of deoxynivalenol and its derivatives (batch and single kernel) using gas chromatography/mass spectrometry. J. Agric. Food Chem. 46:1414-1418.

Nganje, W. E., Bangsund, D. A., Leistritz, F. L., Wilson, W. W., Tiapo, N. M. 2002. Estimating the economic impact of a crop disease: the case of fusarium head blight in U.S. wheat and barley. In: Canty, S., Lewis, J., Siler, L., Ward, R. (eds) 
Proceedings of the 2002 National Fusarium head blight forum, December 7-9, Erlanger, KY.

Peiris, K. H. S., Pumphrey, M. O., Dong, Y., Maghirang, E. B., Berzonsky, W., Dowell, F. E. 2010. Near-infrared spectroscopy method for identification of Fusarium head blight damage and prediction of deoxynivalenol in single wheat kernels. Cereal Chem. 87: 511- 517.

Pumphrey, M. O., Bernardo, R., Anderson, J. 2007. Validating the Fhb1 QTL for Fusarium Head Blight Resistance in Near-Isogenic Wheat Lines Developed from Breeding Populations. Crop Sci. 47:200-206.

Röder, M. S., Korzun, V., Wendehake, K., Plaschke, J., Tixier, M. H., Leroy, P., Ganal, M. 1998. A microsatellite map of wheat. Genetics 149:2007-2023

Ruckenbauer, P., Buerstmayr, H., Lemmens, M. 2001. Present strategies in resistance breeding against scab (Fusarium spp.). Euphytica 119:121-127.

Rudd, J. C., Horsley, R. D., McKendry, A. L., Elias, E. M. 2001. Host plant resistance genes for Fusarium head blight: sources, mechanisms and utility in conventional breeding systems. Crop Sci. 41:620-627.

Smith, N., Guttieri, M., Souza, E., Shoots, J., Sorrells, M., Sneller, C. 2011. Identification and validation of QTL for grain quality traits in a cross of soft wheat cultivars Pioneer Brand 25R26 and Foster. Crop Sci. 51:1424-1436.

Sneller , C. H., Paul, P., Guttieri, M. 2010. Characterization of resistance to Fusarium head blight in an Eastern US soft red winter wheat population. Crop Sci. 50:123133. 
Souza, E. J., Griffey, C., Kweon, M., Guttieri, M. J. 2008. Sources of variation for longflow milling. Crop Sci. 48:1432-1440.

Souza, E., Kweon, M., Sturbaum, A., Goss, C. 2011. 2011 research review USDA ARS Soft Wheat Quality Laboratory, Wooster, OH. Available at http://www.ars.usda.gov/SP2UserFiles/Place/36070500/RR2011/2011ResearchRe view.pdf

Souza, E. J., Sneller, C., Gutierri, M. J., Sturbaum, A., Griffey, C., Sorrells, M., Ohm, H., Van Sanford, D. 2012. Basis for selecting soft wheat for end-use quality. Crop Sci. 52:21-31.

Stack, R. 1999. Return of an old problem: Fusarium Head Blight of small grains. APSnet Features. The American Phytopathological Society, St Paul. MN. Available at http://www.apsnet.org/publications/apsnetfeatures/Pages/headblight.aspx

Tibola, C. S., Fernandes, J. M. C., Delanora, R. 2010. Predicting wheat mycotoxin content using near-infrared reflectance spectroscopy. In: Canty, S., Clark, A., Anderson-Scully, A., Ellis, E., Van Sanford, D. (eds) Proceedings of the 2010 National Fusarium Head Blight Forum, December 7-9, Milwaukee, WI.

Van Sanford, D., Anderson, J., Campbell, K., Costa, J., Cregan, P., Griffey, C., Hayes, P., Ward, R. 2001. Discovery and deployment of molecular markers linked to Fusarium head blight resistance: an integrated system for wheat and barley. Crop Sci. 41: 638- 644.

Verges, V. L., Van Sanford, D., Brown-Guedira, G. 2006. Heritability estimates and response to selection for Fusarium head blight resistance in soft red winter wheat. Crop Sci. 46:1587-1594 
Von der Ohe, C., Ebmeyer, E., Korzun, V., Miedaner, T. 2010. Agronomic and quality performance of Winter wheat backcross populations carrying non-adapted Fusarium head blight resistance QTL. Crop Sci. 50:2283-2290.

Yang, Z. P., Yang, X. Y., Huang, D. C. 2000. Improvement of resistance to Fusarium head blight by recurrent selection in an intermating breeding spring wheat population using the dominant male-sterile gene $m s_{2}$. Euphytica 112:79-88. 
Ana L. Balut was born on September 13, 1981 in Buenos Aires, Argentina. She received the Agricultural Engineer degree from the University of Buenos Aires, in December 2007. She worked for Bunge Argentina S.A. at the Fertilizers Department from February 2005 to July 2009. In August of 2009, she was accepted into the Graduate School at the University of Kentucky and served as a graduate research assistant in the Department of Agronomy working in the research area of wheat breeding. 